\title{
SELECTED HYDROLOGIC DATA FOR CEDAR VALLEY, IRON COUNTY, SOUTHWESTERN UTAH
}

1930-2001

U.S. Geological Survey

Open-File Report No. 01-419

Prepared in cooperation with the CENTRAL IRON COUNTY WATER CONSERVANCY DISTRICT; UTAH DEPARTMENT OF NATURAL RESOURCES, DIVISION OF WATER RESOURCES; UTAH DEPARTMENT OF ENVIRONMENTAL QUALITY, DIVISION OF WATER QUALITY; AND ENOCH CITY

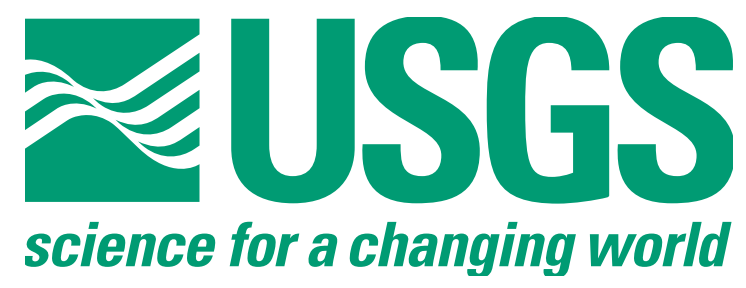





\section{SELECTED HYDROLOGIC DATA FOR CEDAR VALLEY, IRON COUNTY, SOUTHWESTERN UTAH, 1930-2001}

By James H. Howells, James L. Mason, and Bradley A. Slaugh

\section{U.S. GEOLOGICAL SURVEY}

Open-File Report 01-419

Prepared in cooperation with the CENTRAL IRON COUNTY WATER CONSERVANCY DISTRICT; UTAH DEPARTMENT OF NATURAL RESOURCES, DIVISION OF WATER RESOURCES; UTAH DEPARTMENT OF ENVIRONMENTAL QUALITY, DIVISION OF WATER QUALITY; CEDAR CITY; AND ENOCH CITY 


\section{U.S. DEPARTMENT OF THE INTERIOR \\ GALE A. NORTON, Secretary}

\section{U.S. GEOLOGICAL SURVEY}

Charles G. Groat, Director

The use of trade, product, industry, or firm names is for descriptive purposes only and does not imply endorsement by the U.S. Govemment.

For additional information write to:

Copies of this report can be purchased from:

District Chief

U.S. Geological Survey

2329 Orton Circle

Salt Lake City, Utah 84119

U.S. Geological Survey

Branch of Information Services

Box 25286

Denver Federal Center

Denver, Colorado 80225

Additional information about water resources in Utah is available on the World Wide Web at http:// ut.water.usgs.gov 


\section{CONTENTS}

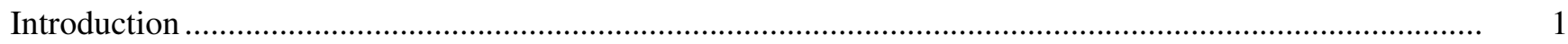

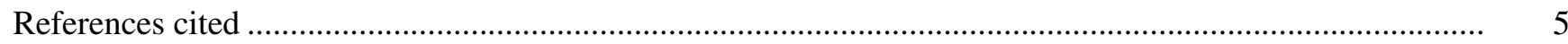

\section{PLATES}

(Plates in pocket)

1. Map showing location of selected wells used for water-level measurements, Cedar Valley, Iron County, southwestern Utah

2. Map showing location of selected wells and surface-water sites where water-quality data were collected, Cedar Valley, Iron County, southwestern Utah

3. Map showing location of selected wells used for water-level measurements, Cedar Valley, Iron County, southwestern Utah

\section{FIGURES}

1. Map showing location of Cedar Valley study area, Iron County, southwestern Utah...................... 2

2. Diagram showing numbering system used for hydrologic-data sites in Utah ................................ 3

3. Hydrograph showing water-level fluctuations in well (C-36-11)8aab-1, Cedar Valley, Iron County, southwestern Utah .

\section{TABLES}

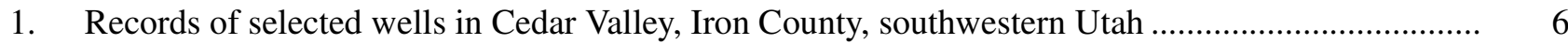

2. Water levels in selected wells in Cedar Valley, Iron County, southwestern Utah ............................ 12

3. Field measurements of $\mathrm{pH}$, specific conductance, and temperature of water from selected wells in Cedar Valley, Iron County, southwestern Utah.

4. Physical properties and results of chemical analyses of water from selected wells and surfacewater sites, Cedar Valley, Iron County, southwestern Utah

5. Discharge, time of measurement, and temperature of water from Coal Creek, Quichapa Ditch, and Shirts Creek, Cedar Valley, Iron County, southwestern Utah 
CONVERSION FACTORS, VERTICAL DATUM, AND ABBREVIATED WATER-QUALITY UNITS

\begin{tabular}{rll}
\hline Multiply & By & To obtain \\
\hline foot $(\mathrm{ft})$ & 0.3048 & meter \\
square mile $\left(\mathrm{mi}^{2}\right)$ & 2.590 & square kilometer \\
\hline
\end{tabular}

The unit cubic feet per second $\left(\mathrm{ft}^{3} / \mathrm{s}\right)$ is used in this report and also can be expressed as $1 \mathrm{ft}^{3} / \mathrm{s}=1.9835$ acre-feet per day.

Water temperature is reported in degrees Celsius $\left({ }^{\circ} \mathrm{C}\right)$, which can be converted to degrees Fahrenheit $\left({ }^{\circ} \mathrm{F}\right)$ by the following equation:

$$
{ }^{\circ} \mathrm{F}=1.8\left({ }^{\circ} \mathrm{C}\right)+32 .
$$

Sea level: In this report, "sea level" refers to the National Geodetic Vertical Datum of 1929—a geodetic datum derived from a general adjustment of the first-order level nets of the United States and Canada, formerly called Sea Level Datum of 1929.

Chemical concentration and water temperature are reported only in metric units. Chemical concentration in water is reported in milligrams per liter $(\mathrm{mg} / \mathrm{L})$ or micrograms per liter $(\mu \mathrm{g} / \mathrm{L})$, which expresses the solute weight per unit volume (liter) of water. One thousand micrograms per liter is equivalent to 1 milligram per liter. For concentrations less than 7,000 milligrams per liter, the numerical value is about the same as for concentrations in parts per million. Specific conductance is reported in microsiemens per centimeter at 25 degrees Celsius $(\mu \mathrm{S} / \mathrm{cm})$. 


\title{
Selected hydrologic data for Cedar Valley, Iron County, southwestern Utah, 1930-2001
}

\author{
By James H. Howells, James L. Mason, and Bradley A. Slaugh
}

\section{INTRODUCTION}

This report presents hydrologic data collected by the U. S. Geological Survey from 1930 to 2001 with emphasis on data collected from 1997 to 2001 as part of a study of ground-water resources in Cedar Valley, Iron County, southwestern Utah (fig. 1). Data collected prior to this study are also presented to show long-term trends. Data were collected during this study in cooperation with the Central Iron County Water Conservancy District; Utah Department of Natural Resources, Division of Water Resources; Utah Department of Environmental Quality, Division of Water Quality; Cedar City; and Enoch City; as part of a study to better understand the ground-water resources of Cedar Valley and to assess possible effects of increased ground-water withdrawal on water quality. Quality of ground water in Cedar Valley is variable and water suppliers need to know if additional water resources can be developed without drawing water of lower quality into public-supply wells.

Cedar Valley is in central Iron County at the transitional boundary between the Basin and Range and Colorado Plateau physiographic provinces described by Hunt (1974) and covers about $570 \mathrm{mi}^{2}$. Additional data from wells west of Cedar Valley and to the south in the vicinity of Kanarraville in the Virgin River drainage (Colorado River Basin) adjacent to the study area are included. Cedar Valley is bounded on the east by the Markagunt Plateau and Red Hills, on the southwest by the Harmony Mountains, on the west by a complex of low hills, and on the north by the Black Mountains. Altitudes in the study area range from about $5,300 \mathrm{ft}$ in Mud Spring Canyon to about 10,400 ft at Blowhard Mountain to the east.

Data collected include well-completion data, water levels in wells, physical properties and chemical constituents of water from wells and streams, and volume of flow in streams and irrigation canals. For com- parison, this report also provides data from earlier years, some of which were published previously by Thomas and Taylor (1946), Bjorkland and others (1977), and Burden and others (2001). Data also are available in Herbert and others (2001) for U.S. Geological Survey gaging station (10242000) on Coal Creek near Cedar City, Utah. The numbering system used in Utah for hydrologic-data sites is illustrated in figure 2 . In this figure, the relation between township, range, and section for a given location and local well number in tables 1-4 and location designation in table 5 is explained.

Ground-water data were collected from existing wells and from new wells completed by private and public owners. Records for 181 selected wells are listed in table 1. Water levels were measured in 161 selected wells and are listed in table 2. Locations for wells with water-level data are shown on plate 1 . Of the 161 wells in which water levels were measured, 6 wells are located in adjacent areas. A hydrograph of water levels in well (C-36-11) 8aab-1 is shown in figure 3.

Field measurements of $\mathrm{pH}$, specific conductance, and temperature of water from selected wells are listed in table 3. Physical properties and chemical analyses of water from 52 selected wells and 3 surface-water sites are listed in table 4. Locations for wells and surfacewater sites with field water-quality measurements and chemical analyses are shown on plate 2. Standard U.S. Geological Survey field procedures were used to collect water-quality data and water samples (Wilde and others, 1998, 1999). Chemical analyses were completed by the U.S. Geological Survey National Water Quality Laboratory.

Surface-water data were collected from Coal Creek, diversions from Coal Creek, and Shirts Creek. Discharge and water temperature from 39 surfacewater sites are listed in table 5. Locations for surfacewater discharge measurement sites are shown on plate 3. Discharge measurements were made on various 


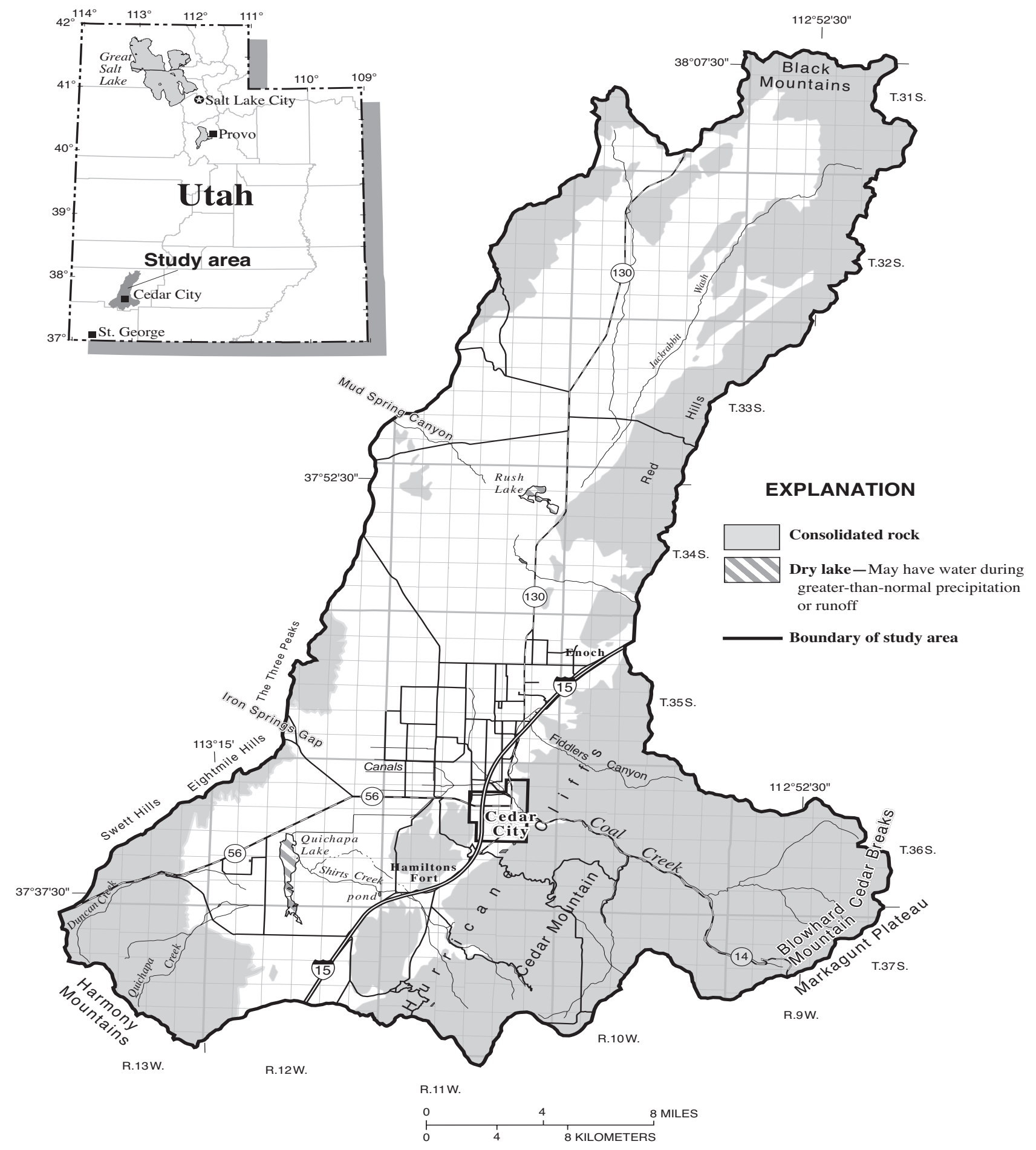

Figure 1. Location of Cedar Valley study area, Iron County, southwestern Utah. 
The system of numbering wells and springs in Utah is based on the cadastral land-survey system of the U.S. Government. The number, in addition to designating the well or spring, describes its position in the land net. The land-survey system divides the State into four quadrants separated by the Salt Lake Base Line and the Salt Lake Meridian. These quadrants are designated by the uppercase letters A, B, $\mathrm{C}$, and $\mathrm{D}$, indicating the northeast, northwest, southwest, and southeast quadrants, respectively. Numbers designating the township and range, in that order, follow the quadrant letter, and all three are enclosed in parentheses. The number after the parentheses indicates the section and is followed by three letters indicating the quarter section, the quarter-quarter section, and the quarter-quarter-quarter section-generally 10 acres for a regular section ${ }^{1}$. The lowercase letters a, b, c, and d indicate, respectively, the northeast, northwest, southwest, and southeast quarters of each subdivision. The number after the letters is the serial number of the well or spring within the 10-acre tract. When the serial number is not preceded by a letter, the number designates a well. When the serial number is preceded by an " $\mathrm{S}$ ", the number designates a spring. A number having all three quarter designations but no serial number indicates a miscellaneous data site other than a well or spring, such as a location for a surface-water measurement site. Thus, (C-35-12)25ddd-1 designates the first well constructed or visited in the southeast quarter of the southeast quarter of the southeast quarter of section 25, T. 35 S., R. $12 \mathrm{~W}$.

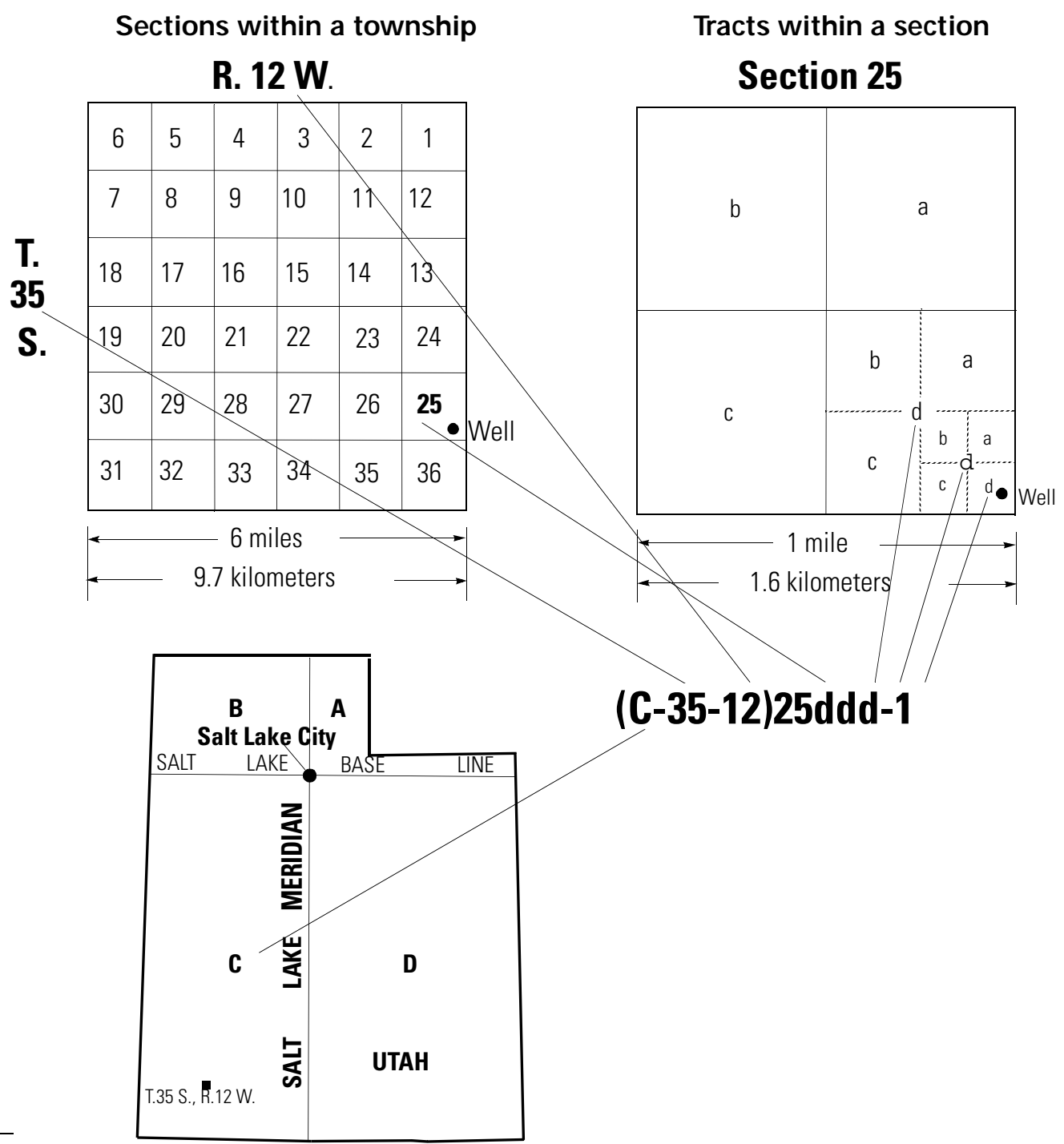

${ }^{1}$ Although the basic land unit, the section, is theoretically 1 square mile, many sections are irregular in size and shape. Such sections are subdivided into 10-acre tracts, generally beginning at the southeast corner, and the surplus or shortage is taken up in the tracts along the north and west sides of the section.

Figure 2. Numbering system used for hydrologic-data sites in Utah. 


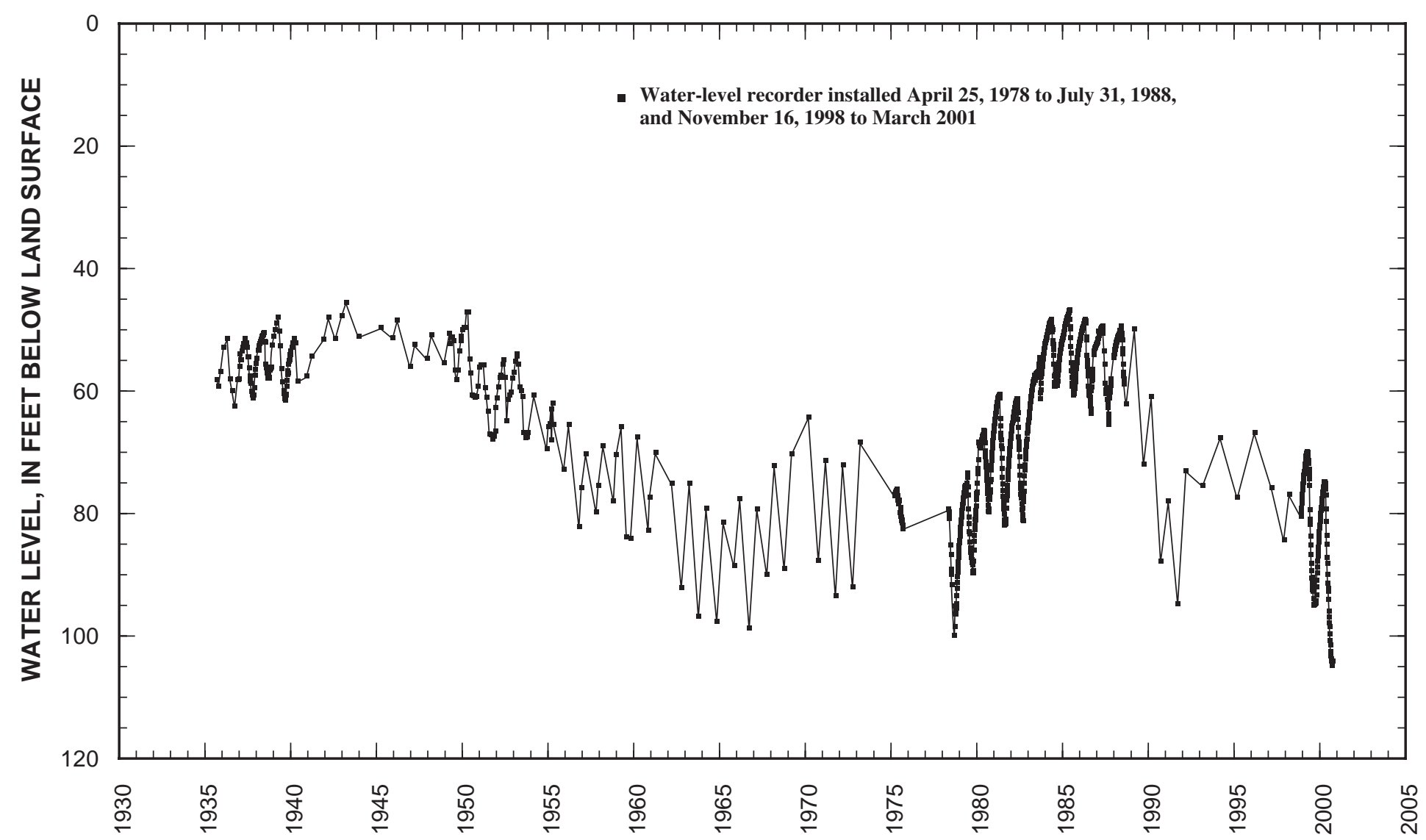

Figure 3. Water-level fluctuations in well (C-36-11)8aab-1, Cedar Valley, Iron County, southwestern Utah. 
reaches of Coal Creek and associated diversions on three occasions. Not all reaches of Coal Creek were measured on each occasion. Discharge measurements were made on Shirts Creek and its tributary on 2 consecutive days. All discharge measurements were made using standard current-meter and volumetric methods of the U.S. Geological Survey (Rantz and others, 1982).

These data could not have been collected without the cooperation of many local residents, landowners, and personnel of Cedar City and Enoch City, who allowed access to their wells for water-level measurements and sampling for chemical analysis, and who provided valuable data and historical information on ground-water resources in Cedar Valley.

\section{REFERENCES CITED}

Bjorklund, L.J., Sumsion, C.T., and Sandberg, G.W., 1977, Selected hydrologic data, Parowan Valley and Cedar City Valley drainage basins, Iron County, Utah: Utah Department of Natural Resources, Division of Water Rights, Utah Basic Data Release No. 28, 55 p.

Burden, C.B., and others, 1999, Ground-water conditions in Utah, spring of 1999: Utah Department of Natural Resources, Division of Water Resources, Cooperative Investigations Report No. 40, 120 p.
Burden, C.B., and others, 2001, Ground-water conditions in Utah, spring of 2001: Utah Department of Natural Resources, Division of Water Resources, Cooperative Investigations Report No. 42, 120 p.

Herbert, L.R., Tibbetts, J.R., Wilberg, D.E., and Allen, D.V., 1999, Water resources data, Utah, water year 1998: U.S. Geological Survey Water-Data Report UT-98-1, $290 \mathrm{p}$.

Herbert, L.R., Wilberg, D.E., Tibbetts, J.R., and Allen, D.V., 2001, Water resources data, Utah, water year 2000: U.S. Geological Survey Water-Data Report UT-00-1, 380 p.

Hunt, C.B., 1974, Natural regions of the United States and Canada: San Francisco, W.H. Freeman, 725 p.

Rantz, S.E., and others, 1982, Measurement and computation of streamflow: Volume 1. Measurement of stage and discharge: Volume 2, Computation of discharge: U.S. Geological Survey Water-Supply Paper 2175, v. 1, p. 1-284; v. 2, p. 285-631.

Thomas, H.E., and Taylor, G.H., 1946, Geology and ground-water resources of Cedar City and Parowan Valleys, Iron County, Utah: U.S. Geological Survey Water-Supply Paper 993, 210 p.

Wilde, F.D., Radtke, D.B., Gibs, J., and Iwatsubo, R.T., eds., 1998, 1999, National field manual for the collection of water-quality data: U.S. Geological Survey Techniques of Water-Resources Investigations, book 9, chap. A1-A6, variously paged. 
Table 1. Records of selected wells in Cedar Valley, Iron County, southwestern Utah

$[-$, no data available $]$

Local well number: See figure 2 for an explanation of the numbering system used for hydrologic-data sites in Utah.

Primary use of water: I, irrigation; S, stock watering; U, unused; P, public supply; H, domestic; K, mining.

Type of finish: F, gravel with perforations; P, perforation; S, screen; X, open hole; O, open end.

Top or bottom of open interval: Reported in feet below land surface.

Other data available: W, water-level measurements in table 2; F, field measurements of water chemistry in table 3; C, chemical analyses in table 4; G, graph of water level in figure 3.

\begin{tabular}{|c|c|c|c|c|c|c|c|c|c|}
\hline Local well number & $\begin{array}{l}\text { Date well } \\
\text { constructed }\end{array}$ & $\begin{array}{l}\text { Primary use } \\
\text { of water }\end{array}$ & $\begin{array}{l}\text { Altitude of } \\
\text { land surface } \\
\text { (feet) }\end{array}$ & $\begin{array}{c}\text { Depth of } \\
\text { well } \\
\text { (feet) }\end{array}$ & $\begin{array}{c}\text { Diameter of } \\
\text { casing } \\
\text { (inches) }\end{array}$ & $\begin{array}{l}\text { Type of } \\
\text { finish }\end{array}$ & $\begin{array}{c}\text { Top of open } \\
\text { interval } \\
\text { (feet) }\end{array}$ & $\begin{array}{c}\text { Bottom of } \\
\text { open interval } \\
\text { (feet) }\end{array}$ & $\begin{array}{c}\text { Other } \\
\text { data } \\
\text { available }\end{array}$ \\
\hline (C-33-10)31ada-1 & $08-17-1965$ & $\mathrm{I}$ & 5,450 & 225 & 16 & $\mathrm{~F}$ & 40 & 225 & $\mathrm{~W}, \mathrm{~F}, \mathrm{C}$ \\
\hline (C-33-10)31cab-1 & 06-18-1972 & I & 5,428 & 94 & 12 & $\mathrm{P}$ & 52 & 94 & W \\
\hline \multirow[t]{2}{*}{ (C-33-11)30bca-1 } & 07-01-1950 & $\mathrm{S}$ & 5,336 & 78 & 6 & $\mathrm{P}$ & 58 & 63 & $\mathrm{~W}, \mathrm{~F}, \mathrm{C}$ \\
\hline & & & & & & & 68 & 73 & \\
\hline (C-33-11)30ddd-2 & - & $\mathrm{U}$ & 5,353 & - & 12 & - & - & - & $\mathrm{W}$ \\
\hline (C-33-11)31aad-1 & - & $S$ & 5,355 & - & 6 & - & - & - & $\mathrm{W}, \mathrm{F}, \mathrm{C}$ \\
\hline \multirow[t]{3}{*}{ (C-33-11)31aad-2 } & $10-19-2000$ & $S$ & 5,355 & 250 & 8 & $\mathrm{~F}$ & 148 & 156 & $\mathrm{~W}$ \\
\hline & & & & & & & 215 & 218 & \\
\hline & & & & & & & 236 & 245 & \\
\hline (C-33-11)33caa-1 & 1890 & $S$ & 5,369 & 25 & 60 & - & - & - & $\mathrm{W}, \mathrm{F}$ \\
\hline \multirow[t]{10}{*}{ (C-34-10)31caa-1 } & 05-10-1951 & $\mathrm{U}$ & 5,490 & 365 & 12 & $\mathrm{~F}$ & 32 & 47 & $\mathrm{~W}, \mathrm{~F}$ \\
\hline & & & & & & & 54 & 64 & \\
\hline & & & & & & & 69 & 71 & \\
\hline & & & & & & & 177 & 184 & \\
\hline & & & & & & & 187 & 188 & \\
\hline & & & & & & & 260 & 262 & \\
\hline & & & & & & & 271 & 285 & \\
\hline & & & & & & & 296 & 301 & \\
\hline & & & & & & & 306 & 316 & \\
\hline & & & & & & & 322 & 342 & \\
\hline (C-34-11) 1daa-1 & $12-05-1960$ & I & 5,402 & 120 & 14 & $\mathrm{P}$ & - & - & $\mathrm{W}, \mathrm{F}$ \\
\hline (C-34-11) 2dad-1 & - & $\mathrm{S}$ & 5,386 & - & 6 & - & - & - & $\mathrm{F}$ \\
\hline \multirow[t]{5}{*}{ (C-34-11) 3cca-1 } & 06-11-1947 & $\mathrm{U}$ & 5,383 & 345 & 16 & $\mathrm{P}$ & 12 & - & $\mathrm{W}$ \\
\hline & & & & & & & 30 & - & \\
\hline & & & & & & & 52 & - & \\
\hline & & & & & & & 60 & - & \\
\hline & & & & & & & 70 & 76 & \\
\hline (C-34-11) 3ccd-1 & - & $S$ & 5,385 & - & 16 & - & - & - & $\mathrm{W}$ \\
\hline (C-34-11) 3dcb-1 & - & $\mathrm{U}$ & 5,390 & 190 & 16 & $\mathrm{P}$ & - & - & $\mathrm{W}$ \\
\hline (C-34-11) 5dcb-1 & - & $\mathrm{U}$ & 5,382 & 31 & 3.5 & - & - & - & W \\
\hline (C-34-11) 9ccd-1 & $12-01-1970$ & $\mathrm{~S}$ & 5,403 & 130 & 6 & $\mathrm{P}$ & 117 & 127 & $\mathrm{~W}, \mathrm{~F}$ \\
\hline (C-34-11) 9cdc-1 & - & $\mathrm{U}$ & 5,402 & 61 & 4 & - & - & - & $\mathrm{W}$ \\
\hline (C-34-11)11dac-1 & 04-29-1974 & $\mathrm{I}$ & 5,387 & 250 & 16 & $\mathrm{~F}$ & 30 & 105 & $\mathrm{~W}$ \\
\hline (C-34-11)13bcd-1 & 02-26-1970 & $\mathrm{I}$ & 5,400 & 152 & 12 & $\mathrm{~F}$ & 122 & 152 & $\mathrm{~W}$ \\
\hline (C-34-11)14aad-2 & 08-06-1983 & I & 5,395 & 125 & 16 & $\mathrm{~F}$ & 105 & 125 & $\mathrm{~W}, \mathrm{~F}, \mathrm{C}$ \\
\hline (C-34-11)16bcc-1 & 1914 & $\mathrm{~S}$ & 5,408 & 85 & 3 & - & - & - & $\mathrm{W}$ \\
\hline (C-34-11)20dba-1 & 10-13-1955 & $\mathrm{S}$ & 5,430 & 108 & 6 & $\mathrm{P}$ & 46 & 52 & $\mathrm{~W}, \mathrm{~F}$ \\
\hline (C-34-11)21dcd-1 & 06-15-1946 & $\mathrm{S}$ & 5,425 & 168 & 8 & $\mathrm{P}$ & 85 & 168 & $\mathrm{~W}, \mathrm{~F}, \mathrm{C}$ \\
\hline (C-34-11)22bda-1 & - & $\mathrm{S}$ & 5,413 & 52 & 3 & - & - & - & W \\
\hline (C-34-11)23bdd-1 & 08-02-1945 & $\mathrm{S}$ & 5,411 & 302 & 16 & - & - & - & $\mathrm{W}, \mathrm{F}$ \\
\hline (C-34-11)29ddd-1 & 04-03-1940 & $\mathrm{S}$ & 5,542 & 260 & 3 & - & - & - & W \\
\hline (C-34-11)31cca-1 & - & $\mathrm{U}$ & 5,580 & - & 4 & - & - & - & $\mathrm{W}$ \\
\hline (C-34-11)36dbb-1 & 04-30-1957 & I & 5,460 & 247 & 14 & $\mathrm{P}$ & 50 & - & $\mathrm{W}$ \\
\hline (C-34-11)36dcc-2 & 04-03-1952 & I & 5,460 & 143 & 16 & $\mathrm{P}$ & 16 & 130 & $\mathrm{~W}, \mathrm{~F}$ \\
\hline
\end{tabular}


Table 1. Records of selected wells in Cedar Valley, Iron County, southwestern Utah—Continued

\begin{tabular}{|c|c|c|c|c|c|c|c|c|c|}
\hline Local well number & $\begin{array}{c}\text { Date well } \\
\text { constructed }\end{array}$ & $\begin{array}{c}\text { Primary use } \\
\text { of water }\end{array}$ & $\begin{array}{c}\text { Altitude of } \\
\text { land surface } \\
\text { (feet) }\end{array}$ & $\begin{array}{l}\text { Depth of } \\
\text { well } \\
\text { (feet) }\end{array}$ & $\begin{array}{l}\text { Diameter of } \\
\text { casing } \\
\text { (inches) }\end{array}$ & $\begin{array}{l}\text { Type of } \\
\text { finish }\end{array}$ & $\begin{array}{c}\text { Top of open } \\
\text { interval } \\
\text { (feet) }\end{array}$ & $\begin{array}{c}\text { Bottom of } \\
\text { open interval } \\
\text { (feet) }\end{array}$ & $\begin{array}{c}\text { Other } \\
\text { data } \\
\text { available }\end{array}$ \\
\hline (C-34-11)36dcc-3 & $07-22-1976$ & I & 5,465 & 498 & 16 & F & 150 & 498 & $\mathrm{~W}, \mathrm{~F}, \mathrm{C}$ \\
\hline (C-35-10) 7abc-1 & $06-25-1966$ & I & 5,570 & 300 & 14 & $\mathrm{~F}$ & 200 & 300 & $\mathrm{~W}, \mathrm{~F}$ \\
\hline (C-35-10) 7bcd-1 & 08-19-1976 & I & 5,510 & 400 & 16 & $\mathrm{P}$ & 150 & 400 & $\mathrm{~W}$ \\
\hline (C-35-10)18abc-1 & $08-17-1973$ & $\mathrm{P}$ & 5,630 & 400 & 12 & $\mathrm{~F}$ & 140 & 400 & $\mathrm{~F}, \mathrm{C}$ \\
\hline (C-35-10)18bbc-1 & - & $\mathrm{U}$ & 5,536 & 200 & 12 & - & - & - & $\mathrm{W}, \mathrm{F}$ \\
\hline (C-35-10)18bcb-1 & - & I & 5,540 & - & 12 & - & - & - & $\mathrm{W}$ \\
\hline (C-35-11) 1bdc-1 & 09-26-1975 & I & 5,460 & 610 & 16 & $\mathrm{~F}$ & 120 & 610 & $\mathrm{~W}$ \\
\hline (C-35-11) 4aba-1 & - & I & 5,456 & - & 14 & - & - & - & W \\
\hline (C-35-11) 4aba-2 & - & $\mathrm{U}$ & 5,456 & - & 12 & - & - & - & W \\
\hline (C-35-11) 5bbc-1 & 11-10-1942 & S & 5,464 & 218 & 6 & $\mathrm{P}$ & 182 & 218 & $\mathrm{~W}$ \\
\hline (C-35-11) 8ddc-1 & 04-30-1953 & S & 5,492 & 260 & 14 & $\mathrm{P}$ & 30 & 260 & $\mathrm{~W}, \mathrm{~F}$ \\
\hline (C-35-11) 9abc-1 & - & $\mathrm{U}$ & 5,477 & - & 4 & - & - & - & $\mathrm{w}$ \\
\hline (C-35-11) 9dba-1 & 11-10-1972 & $\mathrm{H}$ & 5,485 & 500 & 10 & $\mathrm{~F}$ & 200 & 500 & $\mathrm{~F}, \mathrm{C}$ \\
\hline${ }^{1}(\mathrm{C}-35-11) 11 \mathrm{ccc}-1$ & 04-08-1950 & I & 5,495 & 450 & 12 & - & - & - & W, F, C \\
\hline (C-35-11)11ccd-1 & 06-15-1965 & $\mathrm{U}$ & 5,493 & 450 & 10 & $\mathrm{~F}$ & 200 & 450 & $\mathrm{~W}$ \\
\hline (C-35-11)12add-1 & 09-14-1994 & I & 5,490 & 810 & 16 & F & 200 & 810 & $\mathrm{~W}, \mathrm{~F}, \mathrm{C}$ \\
\hline (C-35-11)12ccc-1 & $02-17-1968$ & I & 5,488 & 700 & 14 & $\mathrm{~F}$ & 100 & 700 & $\mathrm{~W}, \mathrm{~F}$ \\
\hline (C-35-11)12ccc-2 & 1958 & $\mathrm{H}$ & 5,490 & 300 & 6 & - & - & - & $\mathrm{F}, \mathrm{C}$ \\
\hline (C-35-11)12dcd-1 & 06-23-1943 & I & 5,498 & 216 & 12 & $\mathrm{P}$ & 30 & 216 & $\mathrm{~W}$ \\
\hline (C-35-11)12ddd-2 & 1935 & $\mathrm{U}$ & 5,514 & 250 & 12 & $\mathrm{P}$ & 185 & 250 & $\mathrm{~W}$ \\
\hline \multirow[t]{2}{*}{ (C-35-11)13ddb-1 } & 03-14-1960 & S & 5,542 & 263 & 12 & $\mathrm{P}$ & 65 & 142 & $\mathrm{~W}, \mathrm{~F}$ \\
\hline & & & & & & & 150 & 263 & \\
\hline (C-35-11)14bac-1 & 09-15-1976 & I & 5,495 & 501 & 14 & $\mathrm{~F}$ & 200 & 501 & $\mathrm{~W}$ \\
\hline (C-35-11) 14bac-2 & 01-30-2001 & $\mathrm{U}$ & $5,494.5$ & 56 & 2 & s & 46 & 56 & $\mathrm{~W}, \mathrm{~F}, \mathrm{C}$ \\
\hline (C-35-11)14bca-1 & $11-26-1971$ & I & 5,500 & 410 & 10 & $\mathrm{~F}$ & 80 & 410 & $\mathrm{~F}, \mathrm{C}$ \\
\hline \multirow[t]{5}{*}{ (C-35-11)15dab-1 } & 01-13-1961 & I & 5,507 & 272 & 10 & $\mathrm{P}$ & 75 & 90 & $\mathrm{~W}$ \\
\hline & & & & & & & 144 & 146 & \\
\hline & & & & & & & 187 & 188 & \\
\hline & & & & & & & 224 & 227 & \\
\hline & & & & & & & 265 & 272 & \\
\hline (C-35-11)16aab-1 & 09-02-1960 & I & 5,494 & 120 & 6 & $\mathrm{P}$ & 110 & 116 & W, F, C \\
\hline (C-35-11)16ccc-1 & 09-02-1967 & $\mathrm{U}$ & 5,510 & 240 & 8 & $\mathrm{~F}$ & 200 & 240 & $\mathrm{~W}$ \\
\hline (C-35-11)17dcd-1 & 1937 & $\mathrm{U}$ & 5,507 & 200 & 12 & - & - & - & $\mathrm{w}$ \\
\hline \multirow[t]{3}{*}{ (C-35-11)17dcd-2 } & 02-04-1993 & I & 5,510 & 320 & 10 & $\mathrm{~F}$ & 205 & 213 & $\mathrm{~W}, \mathrm{~F}$ \\
\hline & & & & & & & 245 & 270 & \\
\hline & & & & & & & 278 & 318 & \\
\hline (C-35-11)19bad-1 & - & S & 5,505 & - & 12 & - & - & - & $\mathrm{W}$ \\
\hline \multirow[t]{3}{*}{ (C-35-11)19dbb-1 } & 03-10-1998 & $\mathrm{H}$ & 5,505 & 530 & 8 & $\mathrm{P}$ & 263 & 265 & F, C \\
\hline & & & & & & & 441 & 444 & \\
\hline & & & & & & & 487 & 528 & \\
\hline \multirow[t]{6}{*}{ (C-35-11)19dcc-1 } & $11-29-1995$ & I & 5,510 & 477 & 12 & $\mathrm{P}$ & 338 & 340 & $\mathrm{~W}, \mathrm{~F}$ \\
\hline & & & & & & & 362 & 363 & \\
\hline & & & & & & & 382 & 384 & \\
\hline & & & & & & & 406 & 407 & \\
\hline & & & & & & & 414 & 415 & \\
\hline & & & & & & & 431 & 435 & \\
\hline (C-35-11)20dda-1 & $11-15-1980$ & I & 5,530 & 712 & 16 & $\mathrm{~F}$ & 132 & 712 & $\mathrm{~W}, \mathrm{~F}$ \\
\hline (C-35-11)21 abd-1 & 07-19-1975 & I & 5,520 & 317 & 10 & $\mathrm{P}$ & 150 & 315 & $\mathrm{~W}, \mathrm{~F}$ \\
\hline \multirow[t]{5}{*}{ (C-35-11)21cdb-2 } & 06-01-1967 & I & 5,533 & 251 & 12 & $\mathrm{~F}$ & 60 & 95 & $\mathrm{~W}$ \\
\hline & & & & & & & 135 & 139 & \\
\hline & & & & & & & 157 & 173 & \\
\hline & & & & & & & 192 & 203 & \\
\hline & & & & & & & 219 & 251 & \\
\hline (C-35-11)21dbd-2 & 06-12-1940 & I & 5,534 & 232 & 16 & - & - & - & $\mathrm{W}, \mathrm{F}$ \\
\hline
\end{tabular}


Table 1. Records of selected wells in Cedar Valley, Iron County, southwestern Utah—Continued

\begin{tabular}{|c|c|c|c|c|c|c|c|c|c|}
\hline Local well number & $\begin{array}{l}\text { Date well } \\
\text { constructed }\end{array}$ & $\begin{array}{l}\text { Primary use } \\
\text { of water }\end{array}$ & $\begin{array}{l}\text { Altitude of } \\
\text { land surface } \\
\text { (feet) }\end{array}$ & $\begin{array}{l}\text { Depth of } \\
\text { well } \\
\text { (feet) }\end{array}$ & $\begin{array}{l}\text { Diameter of } \\
\text { casing } \\
\text { (inches) }\end{array}$ & $\begin{array}{l}\text { Type of } \\
\text { finish }\end{array}$ & $\begin{array}{c}\text { Top of open } \\
\text { interval } \\
\text { (feet) }\end{array}$ & $\begin{array}{l}\text { Bottom of } \\
\text { open interval } \\
\text { (feet) }\end{array}$ & $\begin{array}{c}\text { Other } \\
\text { data } \\
\text { available }\end{array}$ \\
\hline (C-35-11)23bdd-1 & - & i & 5,550 & 385 & 14 & - & - & - & $\mathrm{W}$ \\
\hline (C-35-11)24aab-1 & - & I & 5,590 & 800 & 16 & - & - & - & $\mathrm{W}, \mathrm{F}$ \\
\hline (C-35-11)24bcc-1 & 06-10-1982 & I & 5,585 & 267 & 8 & $\mathrm{~F}$ & 102 & 267 & $\mathrm{~W}$ \\
\hline (C-35-11)25bcc-1 & 07-27-1999 & $\mathrm{U}$ & 5,735 & 318 & 4 & S & 238 & 318 & $\mathrm{~W}, \mathrm{~F}, \mathrm{C}$ \\
\hline (C-35-11)26acd-1 & 11-03-1968 & I & 5,670 & 700 & 16 & F & 140 & 400 & $\mathrm{~W}, \mathrm{~F}, \mathrm{C}$ \\
\hline (C-35-11)26dac-1 & 09-25-1968 & $\mathrm{H}$ & 5,740 & 307 & 6 & $\mathrm{~F}$ & 260 & 307 & $\mathrm{~W}$ \\
\hline \multirow[t]{3}{*}{ (C-35-11)27acc-1 } & 1930 & I & 5,556 & 114 & 12 & $P$ & 47 & 54 & $\mathrm{~W}, \mathrm{~F}$ \\
\hline & & & & & & & 74 & 76 & \\
\hline & & & & & & & 89 & 113 & \\
\hline (C-35-11)27bbc-1 & 1934 & I & 5,547 & 117 & 12 & $\mathrm{P}$ & - & - & $\mathrm{W}, \mathrm{F}$ \\
\hline (C-35-11)27bca-1 & 07-31-1974 & I & 5,550 & 200 & 8 & $\mathrm{P}$ & 170 & 200 & $\mathrm{~W}, \mathrm{~F}$ \\
\hline (C-35-11)27caa-1 & 09-30-1991 & I & 5,560 & 261 & 12 & $\mathrm{~F}$ & 120 & 261 & $\mathrm{~W}$ \\
\hline \multirow[t]{2}{*}{ (C-35-11)27dbb-1 } & 08-19-1960 & I & 5,560 & 156 & 14 & $\mathrm{P}$ & 82 & 126 & $\mathrm{~W}, \mathrm{~F}, \mathrm{C}$ \\
\hline & & & & & & & 131 & 156 & \\
\hline (C-35-11)28aac-2 & 09-05-1977 & I & 5,548 & 354 & 14 & F & - & - & $\mathrm{W}, \mathrm{F}, \mathrm{C}$ \\
\hline (C-35-11)28dac-1 & - & I & 5,562 & 162 & 12 & - & - & - & $\mathrm{W}$ \\
\hline (C-35-11)29acd-1 & - & I & 5,543 & 300 & 14 & - & - & - & W \\
\hline (C-35-11)29add-1 & 08-31-1950 & I & 5,545 & 418 & 12 & - & - & - & F, C \\
\hline (C-35-11)29bdc-1 & $12-29-1997$ & I & 5,535 & 602 & 12 & $\mathrm{~F}$ & 202 & 602 & $\mathrm{~W}$ \\
\hline (C-35-11)29cca-1 & $11-20-1996$ & I & 5,540 & 609 & 12 & F & 50 & 609 & $\mathrm{~W}, \mathrm{~F}$ \\
\hline$(\mathrm{C}-35-11) 29 \mathrm{dbd}-2$ & 08-26-1984 & I & 5,550 & 350 & 12 & $\mathrm{~F}$ & - & - & W, F, C \\
\hline (C-35-11)30caa-1 & - & I & 5,520 & - & - & - & - & - & $\mathrm{F}, \mathrm{C}$ \\
\hline \multirow[t]{5}{*}{ (C-35-11)31acd-1 } & 1919 & & 5,533 & 248 & 12 & $\mathrm{P}$ & 81 & 87 & $\mathrm{~W}$ \\
\hline & & & & & & & 168 & 175 & \\
\hline & & & & & & & 200 & 202 & \\
\hline & & & & & & & 222 & 227 & \\
\hline & & & & & & & 242 & 248 & \\
\hline (C-35-11)31 acd-2 & 05-19-1951 & I & 5,536 & 472 & 12 & - & - & - & W, F, C \\
\hline (C-35-11)31bac-1 & 03-16-1995 & I & 5,520 & 760 & 16 & $\mathrm{~F}$ & 280 & 760 & $\mathrm{~W}$ \\
\hline \multirow[t]{5}{*}{${ }^{2}(\mathrm{C}-35-11) 31 \mathrm{dbd}-1$} & 04-11-1977 & I & 5,535 & 298 & 12 & $\mathrm{P}$ & 172 & 182 & $\mathrm{~W}, \mathrm{~F}, \mathrm{C}$ \\
\hline & & & & & & & 188 & 194 & \\
\hline & & & & & & & 222 & 237 & \\
\hline & & & & & & & 248 & 255 & \\
\hline & & & & & & & 255 & 265 & \\
\hline (C-35-11)32aad-1 & 09-07-1955 & I & 5,560 & 147 & 12 & - & - & - & W \\
\hline (C-35-11)32abd-1 & 09-07-1955 & I & 5,553 & 256 & 16 & $\mathrm{P}$ & 75 & 256 & $\mathrm{w}$ \\
\hline \multirow[t]{3}{*}{ (C-35-11)32bda-1 } & 04-19-1975 & I & 5,547 & 386 & 16 & $\mathrm{P}$ & 216 & 223 & $\mathrm{~W}, \mathrm{~F}$ \\
\hline & & & & & & & 239 & 242 & \\
\hline & & & & & & & 250 & 384 & \\
\hline (C-35-11)32dba-1 & 1934 & I & 5,555 & 132 & 12 & $\mathrm{P}$ & 100 & 128 & $\mathrm{~W}, \mathrm{~F}$ \\
\hline (C-35-11)33aac-1 & - & I & 5,576 & 236 & 14 & $P$ & 138 & 236 & $\mathrm{~W}, \mathrm{~F}, \mathrm{C}$ \\
\hline (C-35-11)33abd-1 & 02-25-1956 & I & 5,578 & 217 & 14 & $P$ & 85 & 217 & $\mathrm{~F}, \mathrm{C}$ \\
\hline (C-35-11)33bad-1 & $12-01-1972$ & I & 5,573 & 252 & 16 & $\mathrm{P}$ & 100 & 250 & $\mathrm{~F}$ \\
\hline (C-35-11)33bcc-1 & 03-20-1940 & I & 5,563 & 200 & 16 & $P$ & 70 & 200 & $\mathrm{~W}, \mathrm{~F}$ \\
\hline (C-35-11)33ccd-1 & $12-30-1971$ & I & 5,575 & 217 & 12 & $P$ & 100 & 215 & $\mathrm{~W}, \mathrm{~F}, \mathrm{C}$ \\
\hline \multirow[t]{6}{*}{ (C-35-11)33dbd-2 } & $05-12-1951$ & $\mathrm{U}$ & 5,585 & 206 & 14 & $P$ & 95 & 102 & $\mathrm{~W}$ \\
\hline & & & & & & & 105 & 112 & \\
\hline & & & & & & & 143 & 147 & \\
\hline & & & & & & & 170 & 179 & \\
\hline & & & & & & & 181 & 192 & \\
\hline & & & & & & & 198 & 200 & \\
\hline (C-35-11)34abb-1 & 07-29-1961 & I & 5,575 & 241 & 16 & $\mathrm{P}$ & 108 & 240 & $\mathrm{w}$ \\
\hline (C-35-11)34dbb-1 & 01-10-1976 & K & 5,600 & 152 & 12 & $\mathrm{~F}$ & - & - & $\mathrm{W}, \mathrm{F}, \mathrm{C}$ \\
\hline (C-35-12)18ddd-4 & $10-15-1964$ & $\mathrm{~K}$ & 5,382 & 300 & 12 & $P$ & 50 & 300 & W \\
\hline
\end{tabular}


Table 1. Records of selected wells in Cedar Valley, Iron County, southwestern Utah—Continued

\begin{tabular}{|c|c|c|c|c|c|c|c|c|c|}
\hline Local well number & $\begin{array}{l}\text { Date well } \\
\text { constructed }\end{array}$ & $\begin{array}{c}\text { Primary use } \\
\text { of water }\end{array}$ & $\begin{array}{c}\text { Altitude of } \\
\text { land surface } \\
\text { (feet) }\end{array}$ & $\begin{array}{c}\text { Depth of } \\
\text { well } \\
\text { (feet) }\end{array}$ & $\begin{array}{l}\text { Diameter of } \\
\text { casing } \\
\text { (inches) }\end{array}$ & $\begin{array}{l}\text { Type of } \\
\text { finish }\end{array}$ & $\begin{array}{l}\text { Top of open } \\
\text { interval } \\
\text { (feet) }\end{array}$ & $\begin{array}{c}\text { Bottom of } \\
\text { open interval } \\
\text { (feet) }\end{array}$ & $\begin{array}{c}\text { Other } \\
\text { data } \\
\text { available }\end{array}$ \\
\hline (C-35-12)20dab-1 & 11-28-1969 & $\mathrm{U}$ & 5,420 & 175 & 16 & $F$ & 12 & 175 & $\mathrm{~W}$ \\
\hline \multirow[t]{5}{*}{ (C-35-12)25ddd-1 } & 08-14-1968 & S & 5,513 & 200 & 8 & $\mathrm{P}$ & 46 & 51 & $\mathrm{~W}, \mathrm{~F}$ \\
\hline & & & & & & & 56 & 62 & \\
\hline & & & & & & & 95 & 102 & \\
\hline & & & & & & & 110 & 122 & \\
\hline & & & & & & & 195 & 200 & \\
\hline \multirow[t]{2}{*}{ (C-35-12)26bca-1 } & 07-08-1997 & S & 5,480 & 220 & 8 & $\mathrm{P}$ & 100 & 140 & $\mathrm{~W}, \mathrm{~F}, \mathrm{C}$ \\
\hline & & & & & & & 180 & 220 & \\
\hline (C-35-12)27bbd-1 & $06-12-1958$ & K & 5,465 & 270 & 12 & $\mathrm{P}$ & 92 & 270 & $\mathrm{~W}$ \\
\hline (C-35-12)27bca-1 & $05-29-1968$ & K & 5,460 & 247 & 16 & F & 35 & 147 & W \\
\hline (C-35-12)27bcc-1 & 08-03-1968 & $\mathrm{K}$ & 5,460 & 250 & 16 & F & 20 & 250 & $\mathrm{~W}, \mathrm{~F}$ \\
\hline \multirow[t]{3}{*}{ (C-35-12)34dcd-2 } & $10-10-1959$ & I & 5,480 & 144 & 14 & $\mathrm{P}$ & 72 & 76 & $\mathrm{~W}$ \\
\hline & & & & & & & 90 & 91 & \\
\hline & & & & & & & 112 & 120 & \\
\hline \multirow[t]{3}{*}{ (C-35-12)36аaа-1 } & 03-22-1996 & I & 5,515 & 698 & 16 & F & 212 & 500 & $\mathrm{~W}, \mathrm{~F}$ \\
\hline & & & & & & & 540 & 578 & \\
\hline & & & & & & & 618 & 698 & \\
\hline (C-35-12)36caa-1 & 03-31-1982 & I & 5,505 & 704 & 16 & F & 214 & 704 & $\mathrm{~W}, \mathrm{~F}, \mathrm{C}$ \\
\hline (C-35-12)36саa-2 & 02-02-2001 & $\mathrm{U}$ & $5,404.7$ & 75 & 2 & S & 65 & 75 & W, F, C \\
\hline (C-35-12)36ddd-1 & 03-28-1994 & I & 5,515 & 595 & 16 & F & 200 & 595 & $\mathrm{~W}, \mathrm{~F}, \mathrm{C}$ \\
\hline (C-36-11) 3bda-1 & $10-14-1980$ & $\mathrm{~K}$ & 5,640 & 310 & 16 & $\mathrm{P}$ & 140 & 310 & $\mathrm{~W}$ \\
\hline \multirow[t]{2}{*}{ (C-36-11) 5abb-1 } & 08-29-1940 & I & 5,558 & 212 & 10 & $\mathrm{P}$ & 171 & 173 & $\mathrm{~F}$ \\
\hline & & & & & & & 193 & 200 & \\
\hline (C-36-11) 5aca-1 & 05-10-1939 & I & 5,565 & 166 & 16 & $\mathrm{P}$ & 70 & 166 & F, C \\
\hline (C-36-11) 5acc-1 & - & I & 5,556 & - & 14 & - & - & - & $\mathrm{F}$ \\
\hline (C-36-11) 5cac-1 & 04-01-1934 & I & 5,548 & 220 & 12 & $\mathrm{P}$ & 70 & 220 & $\mathrm{~F}$ \\
\hline (C-36-11) 5cba-1 & 03-01-1935 & I & 5,550 & 230 & 12 & $\mathrm{P}$ & 70 & 230 & $\mathrm{~W}, \mathrm{~F}$ \\
\hline (C-36-11) 5dab-1 & 03-23-1986 & K & 5,570 & 200 & 8 & $\mathrm{~F}$ & 100 & 200 & $\mathrm{~F}, \mathrm{C}$ \\
\hline (C-36-11) 5dcc-1 & 05-21-1957 & I & 5,555 & 204 & 16 & $\mathrm{P}$ & 100 & 180 & $\mathrm{w}$ \\
\hline (C-36-11) 7aaa-2 & - & $\mathrm{H}$ & 5,535 & 300 & 10 & - & - & - & $\mathrm{F}, \mathrm{C}$ \\
\hline \multirow[t]{3}{*}{ (C-36-11) 7aba-1 } & 04-27-1965 & I & 5,532 & 352 & 16 & $\mathrm{P}$ & 102 & 108 & $\mathrm{~W}, \mathrm{~F}$ \\
\hline & & & & & & & 157 & 186 & \\
\hline & & & & & & & 283 & 345 & \\
\hline \multirow[t]{2}{*}{ (C-36-11) 7cab-1 } & 09-05-1961 & I & 5,522 & 358 & 14 & $\mathrm{P}$ & 267 & 272 & $\mathrm{~W}, \mathrm{~F}, \mathrm{C}$ \\
\hline & & & & & & & 295 & 355 & \\
\hline \multirow[t]{3}{*}{ (C-36-11) 8aab-1 } & 1935 & $\mathrm{U}$ & 5,563 & 220 & 8 & $\mathrm{P}$ & 130 & 163 & W, F, G \\
\hline & & & & & & & 200 & 202 & \\
\hline & & & & & & & 212 & 218 & \\
\hline (C-36-11) 8abd-1 & $10-09-1972$ & I & 5,562 & 265 & 16 & $\mathrm{P}$ & 125 & 265 & $\mathrm{~W}$ \\
\hline (C-36-11) 8aca-1 & - & I & 5,563 & 210 & 16 & $\mathrm{P}$ & 125 & 210 & $\mathrm{~W}$ \\
\hline (C-36-11) 8bbb-1 & 07-10-1972 & I & 5,537 & 300 & 10 & $\mathrm{~F}$ & 100 & 300 & $\mathrm{w}$ \\
\hline \multirow[t]{5}{*}{ (C-36-11) 8cbb-1 } & $11-30-1955$ & I & 5,539 & 240 & 14 & $P$ & 52 & 62 & W, F \\
\hline & & & & & & & 80 & 86 & \\
\hline & & & & & & & 121 & 124 & \\
\hline & & & & & & & 164 & 165 & \\
\hline & & & & & & & 208 & 230 & \\
\hline (C-36-11) 8dab-1 & 05-02-1924 & S & 5,590 & 192 & 10 & - & - & - & $\mathrm{W}$ \\
\hline (C-36-11)11bac-1 & 07-14-1968 & I & 5,758 & 644 & 16 & $\mathrm{P}$ & 221 & 623 & $\mathrm{~W}, \mathrm{~F}, \mathrm{C}$ \\
\hline (C-36-11)13adb-1 & 05-31-1980 & $\mathrm{U}$ & 6,020 & 670 & 16 & $\mathrm{X}$ & 100 & 670 & $\mathrm{~W}$ \\
\hline (C-36-11)18aaa-1 & $10-15-1985$ & I & 5,530 & 505 & 10 & $\mathrm{~F}$ & 250 & 505 & $\mathrm{~W}$ \\
\hline (C-36-11)18baa-1 & 06-15-1977 & I & 5,520 & 390 & 16 & $\mathrm{~F}$ & 125 & 390 & W, F \\
\hline (C-36-11)18bca-2 & 01-26-1962 & I & 5,510 & 320 & 12 & $\mathrm{P}$ & 285 & 320 & $\mathrm{~W}, \mathrm{~F}$ \\
\hline (C-36-11)18bdd-1 & 05-28-1994 & I & 5,515 & 390 & 16 & F & 110 & 390 & W, F, C \\
\hline (C-36-11)30acc-2 & 08-29-1996 & $\mathrm{H}$ & 5,620 & 600 & 12 & $\mathrm{P}$ & 220 & 360 & $\mathrm{~W}$ \\
\hline
\end{tabular}


Table 1. Records of selected wells in Cedar Valley, Iron County, southwestern Utah—Continued

\begin{tabular}{|c|c|c|c|c|c|c|c|c|c|}
\hline Local well number & $\begin{array}{l}\text { Date well } \\
\text { constructed }\end{array}$ & $\begin{array}{l}\text { Primary use } \\
\text { of water }\end{array}$ & $\begin{array}{l}\text { Altitude of } \\
\text { land surface } \\
\text { (feet) }\end{array}$ & $\begin{array}{l}\text { Depth of } \\
\text { well } \\
\text { (feet) }\end{array}$ & $\begin{array}{l}\text { Diameter of } \\
\text { casing } \\
\text { (inches) }\end{array}$ & $\begin{array}{l}\text { Type of } \\
\text { finish }\end{array}$ & $\begin{array}{c}\text { Top of open } \\
\text { interval } \\
\text { (feet) }\end{array}$ & $\begin{array}{c}\text { Bottom of } \\
\text { open interval } \\
\text { (feet) }\end{array}$ & $\begin{array}{c}\text { Other } \\
\text { data } \\
\text { available }\end{array}$ \\
\hline & & & & & & & 430 & 600 & \\
\hline (C-36-11)31 abc-1 & 03-06-1991 & $\mathrm{H}$ & 5,650 & 400 & 8 & $\mathrm{P}$ & 320 & 400 & F, C \\
\hline (C-36-11)31daa-1 & 04-08-1992 & S & 5,700 & 400 & 8 & $\mathrm{~F}$ & 280 & 320 & $\mathrm{~W}$ \\
\hline \multirow[t]{2}{*}{ (C-36-11)31daa-2 } & 05-08-1992 & S & 5,715 & 420 & 8 & F & 300 & 340 & $\mathrm{~W}$ \\
\hline & & & & & & & 380 & 420 & \\
\hline$(\mathrm{C}-36-12) 1 \mathrm{dcc}-2$ & - & $\mathrm{U}$ & 5,505 & - & 4 & - & - & - & W \\
\hline (C-36-12) 2aac-1 & - & S & 5,494 & 408 & 12 & - & - & - & $\mathrm{W}$ \\
\hline (C-36-12) 2dbc-1 & 08-17-1985 & I & 5,490 & 815 & 12 & $\mathrm{~F}$ & 355 & 815 & $\mathrm{~F}, \mathrm{C}$ \\
\hline (C-36-12) 2dbc-2 & 08-20-1975 & I & 5,492 & 450 & 12 & $\mathrm{~F}$ & 160 & 450 & $\mathrm{~W}$ \\
\hline (C-36-12) $2 \mathrm{dbc}-3$ & 03-13-1983 & I & 5,490 & 457 & 10 & $\mathrm{~F}$ & 125 & 450 & W \\
\hline \multirow[t]{2}{*}{ (C-36-12) 3aad-1 } & 08-07-1977 & S & 5,485 & 200 & 8 & F & 70 & 110 & W \\
\hline & & & & & & & 160 & 200 & \\
\hline (C-36-12) 3aad-2 & 08-31-1998 & I & 5,485 & 357 & 6 & F & 157 & 357 & $\mathrm{~W}, \mathrm{~F}, \mathrm{C}$ \\
\hline (C-36-12) 9aaa-1 & 05-29-1939 & $\mathrm{U}$ & 5,475 & 257 & 6 & $\mathrm{P}$ & 208 & 257 & $\mathrm{~W}$ \\
\hline \multirow[t]{4}{*}{ (C-36-12) 9aac-1 } & 07-26-1966 & I & 5,465 & 250 & 14 & $P$ & 65 & 90 & F, C \\
\hline & & & & & & & 115 & 121 & \\
\hline & & & & & & & 135 & 162 & \\
\hline & & & & & & & 168 & 230 & \\
\hline (C-36-12)10aaa-1 & 09-11-1951 & $\mathrm{U}$ & 5,470 & 245 & 6 & - & - & - & W \\
\hline (C-36-12)10dda-1 & - & $\mathrm{U}$ & 5,476 & & 4 & - & - & - & W \\
\hline (C-36-12)12dba-1 & 04-01-1925 & I & 5,509 & 600 & 10 & $\mathrm{P}$ & 200 & 560 & W, F, C \\
\hline (C-36-12)15dda- 1 & - & $\mathrm{U}$ & 5,475 & - & 4 & - & - & - & W \\
\hline (C-36-12)16bba-1 & 04-05-1957 & $\mathrm{U}$ & 5,470 & 85 & 6 & $\mathrm{O}$ & - & - & $\mathrm{W}$ \\
\hline (C-36-12)20acc-1 & 04-15-1978 & I & 5,495 & 600 & 16 & $\mathrm{~F}$ & 150 & 600 & $\mathrm{~W}, \mathrm{~F}$ \\
\hline (C-36-12)20ddc-1 & 1916 & $\mathrm{U}$ & 5,476 & - & 2 & - & - & - & $\mathrm{w}$ \\
\hline \multirow[t]{5}{*}{ (C-36-12)21 cbb-1 } & 03-07-1967 & S & 5,470 & 228 & 16 & $\mathrm{P}$ & 55 & 83 & $\mathrm{~W}, \mathrm{~F}, \mathrm{C}$ \\
\hline & & & & & & & 110 & 114 & \\
\hline & & & & & & & 140 & 144 & \\
\hline & & & & & & & 178 & 180 & \\
\hline & & & & & & & 201 & 203 & \\
\hline (C-36-12)25bdd-1 & 11-13-1956 & I & 5,540 & 300 & 14 & $\mathrm{P}$ & 90 & - & $\mathrm{W}, \mathrm{F}$ \\
\hline (C-36-12)25cda-1 & 04-29-1971 & I & 5,550 & 370 & 16 & $\mathrm{~F}$ & 120 & 370 & W \\
\hline$(\mathrm{C}-36-12) 28 \mathrm{ccc}-1$ & - & $\mathrm{U}$ & 5,465 & - & 2 & - & - & - & W \\
\hline \multirow[t]{4}{*}{ (C-36-12)32dcc-1 } & 08-16-1960 & I & 5,498 & 276 & 16 & $\mathrm{P}$ & 55 & 61 & $\mathrm{~W}, \mathrm{~F}$ \\
\hline & & & & & & & 128 & 135 & \\
\hline & & & & & & & 168 & 185 & \\
\hline & & & & & & & 207 & 230 & \\
\hline (C-36-12)32dcd-1 & 05-15-1964 & $\mathrm{U}$ & 5,482 & 305 & 16 & $\mathrm{P}$ & 70 & 286 & W \\
\hline (C-36-12)33bcc-1 & - & $\mathrm{H}$ & 5,465 & 200 & 8 & - & - & - & W \\
\hline (C-36-12)35adc-1 & 07-06-1968 & I & 5,510 & 700 & 14 & $\mathrm{~F}$ & - & - & $\mathrm{W}$ \\
\hline (C-36-12)36ada-1 & $12-04-1965$ & I & 5,604 & 325 & 14 & $\mathrm{~F}$ & 150 & 325 & $\mathrm{w}$ \\
\hline \multirow[t]{6}{*}{ (C-36-12)36adb-1 } & $10-03-1951$ & I & 5,595 & 307 & 12 & $\mathrm{P}$ & 108 & 113 & F, C \\
\hline & & & & & & & 130 & 150 & \\
\hline & & & & & & & 190 & 196 & \\
\hline & & & & & & & 204 & 206 & \\
\hline & & & & & & & 218 & 235 & \\
\hline & & & & & & & 258 & 282 & \\
\hline \multirow[t]{3}{*}{ (C-37-12) 5acc-1 } & 06-24-1959 & $\mathrm{U}$ & 5,504 & 216 & 16 & $\mathrm{P}$ & 55 & 90 & $\mathrm{~W}$ \\
\hline & & & & & & & 130 & 145 & \\
\hline & & & & & & & 175 & 205 & \\
\hline (C-37-12) 5 acc-2 & 05-15-1993 & I & 5,505 & 458 & 16 & $\mathrm{~F}$ & 158 & 458 & $\mathrm{~W}, \mathrm{~F}, \mathrm{C}$ \\
\hline (C-37-12) 5bbb-1 & 07-16-1965 & $\mathrm{U}$ & 5,532 & 300 & 16 & $\mathrm{~F}$ & 90 & 300 & $\mathrm{~W}$ \\
\hline (C-37-12) 5bcb-1 & $05-15-1960$ & $\mathrm{U}$ & 5,542 & 252 & 16 & $\mathrm{P}$ & 72 & 125 & $\mathrm{~W}$ \\
\hline
\end{tabular}


Table 1. Records of selected wells in Cedar Valley, Iron County, southwestern Utah—Continued

\begin{tabular}{|c|c|c|c|c|c|c|c|c|c|}
\hline Local well number & $\begin{array}{c}\text { Date well } \\
\text { constructed }\end{array}$ & $\begin{array}{l}\text { Primary use } \\
\text { of water }\end{array}$ & $\begin{array}{l}\text { Altitude of } \\
\text { land surface } \\
\text { (feet) }\end{array}$ & $\begin{array}{l}\text { Depth of } \\
\text { well } \\
\text { (feet) }\end{array}$ & $\begin{array}{c}\text { Diameter of } \\
\text { casing } \\
\text { (inches) }\end{array}$ & $\begin{array}{l}\text { Type of } \\
\text { finish }\end{array}$ & $\begin{array}{c}\text { Top of open } \\
\text { interval } \\
\text { (feet) }\end{array}$ & $\begin{array}{l}\text { Bottom of } \\
\text { open interval } \\
\text { (feet) }\end{array}$ & $\begin{array}{c}\text { Other } \\
\text { data } \\
\text { available }\end{array}$ \\
\hline & & & & & & & 142 & 144 & \\
\hline & & & & & & & 167 & 187 & \\
\hline (C-37-12) 9acc-1 & 07-18-1959 & I & 5,490 & 186 & 14 & $\mathrm{P}$ & 75 & 180 & W, F, C \\
\hline (C-37-12)11 aaa-1 & 09-29-1953 & $\mathrm{U}$ & 5,490 & 365 & 14 & $\mathrm{P}$ & 25 & - & $\mathrm{W}, \mathrm{F}$ \\
\hline \multirow[t]{4}{*}{ (C-37-12)11 aab-1 } & 06-12-1995 & I & 5,490 & 480 & 16 & F & 120 & 160 & $\mathrm{~W}$ \\
\hline & & & & & & & 200 & 240 & \\
\hline & & & & & & & 280 & 300 & \\
\hline & & & & & & & 320 & 480 & \\
\hline (C-37-12)11dac- 1 & - & I & 5,495 & 236 & 16 & - & - & - & $\mathrm{W}, \mathrm{F}, \mathrm{C}$ \\
\hline \multirow[t]{3}{*}{ (C-37-12)14abc-1 } & $05-19-1950$ & I & 5,480 & 240 & 16 & $\mathrm{P}$ & 189 & 206 & $\mathrm{~W}, \mathrm{~F}$ \\
\hline & & & & & & & 219 & 222 & \\
\hline & & & & & & & 233 & 237 & \\
\hline \multirow[t]{3}{*}{ (C-37-12)14dbc-1 } & 05-19-1950 & I & 5,493 & 240 & 12 & $\mathrm{P}$ & 189 & 206 & $\mathrm{~W}, \mathrm{~F}$ \\
\hline & & & & & & & 219 & 222 & \\
\hline & & & & & & & 233 & 237 & \\
\hline (C-37-12)14dbd-1 & $07-15-1961$ & $\mathrm{U}$ & 5,498 & 237 & 16 & F & 75 & 237 & $\mathrm{~W}$ \\
\hline (C-37-12)23abd-1 & - & I & 5,530 & 276 & 16 & $P$ & - & - & $\mathrm{W}, \mathrm{F}, \mathrm{C}$ \\
\hline (C-37-12)23acb-1 & 1915 & I & 5,512 & 250 & 16 & $\mathrm{P}$ & 96 & 250 & W, F, C \\
\hline \multirow[t]{5}{*}{ (C-37-12)28aac-1 } & 09-11-1978 & S & 5,550 & 576 & 20 & F & 242 & 262 & $\mathrm{~W}$ \\
\hline & & & & & & & 424 & 429 & \\
\hline & & & & & & & 469 & 481 & \\
\hline & & & & & & & 511 & 516 & \\
\hline & & & & & & & 556 & 557 & \\
\hline (C-37-12)34abb-1 & $08-22-1934$ & I & 5,507 & 190 & 14 & - & - & - & $\mathrm{W}, \mathrm{F}, \mathrm{C}$ \\
\hline (C-38-12) 9aab-1 & $10-18-1967$ & $\mathrm{U}$ & 5,407 & 300 & 12 & F & 100 & 300 & W \\
\hline (C-38-12) 9bba-1 & 04-26-1936 & $\mathrm{U}$ & 5,335 & 135 & 12 & $P$ & 40 & 110 & $\mathrm{~W}$ \\
\hline
\end{tabular}

${ }^{1}$ Supersedes well reported as (C-35-11)11ccc-1 by Bjorklund and others (1977).

${ }^{2}$ Previously reported as (C-35-11)31dbb-1 by Burden and others (1999) and Herbert and others (1999). 
Table 2. Water levels in selected wells in Cedar Valley, Iron County, southwestern Utah

Local well number: See figure 2 for an explanation of the numbering system used for hydrologic-data sites in Utah. See plate 1 for location of wells.

Water level: In feet above (-) or below land surface; D, water level from drillers' log; R, water level rounded to th nearest foot.

\begin{tabular}{|c|c|c|c|c|c|c|c|c|}
\hline Local well number & $\begin{array}{c}\text { Altitude of } \\
\text { land } \\
\text { surface } \\
\text { (feet) }\end{array}$ & Date & $\begin{array}{r}\text { Wate } \\
\text { (f }\end{array}$ & & Local well number & $\begin{array}{l}\text { Altitude of } \\
\text { land } \\
\text { surface } \\
\text { (feet) }\end{array}$ & Date & $\begin{array}{c}\text { Water level } \\
\text { (feet) }\end{array}$ \\
\hline (C-33-10)31ada-1 & 5,450 & 08-18-1965 & 50 & $\mathrm{D}$ & (C-33-11)30ddd-2- & tinued & $03-22-1962$ & 36.80 \\
\hline & & $11-18-1998$ & 73.0 & & & & $10-10-1962$ & 37.43 \\
\hline & & 11-01-1999 & 74.0 & & & & 03-25-1963 & 36.86 \\
\hline & & & & & & & $10-28-1963$ & 37.31 \\
\hline (C-33-10)31 cab-1 & 5,428 & 06-18-1972 & 52 & $\mathrm{D}$ & & & 03-19-1964 & 36.87 \\
\hline & & 08-29-1973 & 45 & $\mathrm{R}$ & & & 11-02-1964 & 37.34 \\
\hline & & 03-18-1974 & 44.9 & & & & 03-17-1965 & 36.90 \\
\hline & & 11-18-1998 & 50.6 & & & & 11-03-1965 & 37.42 \\
\hline & & 11-01-1999 & 54.2 & & & & 03-10-1966 & 36.96 \\
\hline & & & & & & & 09-27-1966 & 37.58 \\
\hline (C-33-11)30bca-1 & 5,336 & $06-27-1955$ & 45 & $\mathrm{D}$ & & & 03-08-1967 & 37.08 \\
\hline & & 03-19-1974 & 47 & $\mathrm{R}$ & & & $10-11-1967$ & 37.40 \\
\hline & & 08-26-1998 & 48.0 & & & & $10-08-1968$ & 37.63 \\
\hline & & 11-17-1998 & 47.9 & & & & 03-14-1969 & 37.00 \\
\hline & & 03-15-1999 & 47.7 & & & & 10-08-1969 & 37.62 \\
\hline & & 07-26-1999 & 47.7 & & & & 03-11-1970 & 37.10 \\
\hline & & 11-01-1999 & 47.2 & & & & $10-08-1970$ & 37.60 \\
\hline & & $03-13-2000$ & 48.9 & & & & 03-08-1971 & 37.17 \\
\hline & & & & & & & 09-30-1971 & 37.72 \\
\hline (C-33-11)30ddd- 2 & 5,353 & $12-05-1948$ & 35.7 & & & & 03-08-1972 & 37.22 \\
\hline & & 04-18-1949 & 35.3 & & & & 10-04-1972 & 37.80 \\
\hline & & 12-07-1949 & 35.8 & & & & $03-15-1973$ & 37.28 \\
\hline & & $03-22-1950$ & 35.7 & & & & 10-04-1973 & 37.90 \\
\hline & & $12-06-1950$ & 36.0 & & & & 03-07-1974 & 37.43 \\
\hline & & 03-20-1951 & 35.7 & & & & $10-22-1974$ & 37.98 \\
\hline & & $12-06-1951$ & 36.1 & & & & 03-07-1975 & 37.62 \\
\hline & & 04-02-1952 & 35.7 & & & & $10-08-1975$ & 38.22 \\
\hline & & $12-03-1952$ & 36.3 & & & & 03-02-1976 & 37.92 \\
\hline & & 03-12-1953 & 35.9 & & & & $10-19-1976$ & 38.13 \\
\hline & & $12-07-1953$ & 36.3 & & & & 03-01-1977 & 37.68 \\
\hline & & 03-18-1955 & 36.2 & & & & $10-03-1977$ & 38.28 \\
\hline & & 12-01-1955 & 36.5 & & & & 03-02-1978 & 37.73 \\
\hline & & 03-14-1956 & 36.3 & & & & 03-16-1978 & 37.74 \\
\hline & & $10-22-1956$ & 36.6 & & & & 03-13-1979 & 37.75 \\
\hline & & $12-13-1956$ & 36.4 & & & & $10-02-1979$ & 38.48 \\
\hline & & 03-11-1957 & 36.1 & & & & 03-06-1980 & 37.98 \\
\hline & & $10-24-1957$ & 36.7 & & & & $10-01-1980$ & 38.48 \\
\hline & & $12-03-1957$ & 36.5 & & & & 03-03-1981 & 38.97 \\
\hline & & 03-18-1958 & 36.3 & & & & $10-01-1981$ & 38.61 \\
\hline & & $10-27-1958$ & 36.8 & & & & 03-01-1982 & 38.05 \\
\hline & & $12-16-1958$ & 36.6 & & & & $10-01-1982$ & 38.59 \\
\hline & & 04-08-1959 & 36.3 & & & & 03-01-1983 & 38.00 \\
\hline & & $10-26-1959$ & 37.0 & & & & 09-28-1983 & 38.43 \\
\hline & & $12-05-1959$ & 36.9 & & & & 03-08-1984 & 37.95 \\
\hline & & 03-16-1960 & 36.6 & & & & 09-26-1984 & 38.28 \\
\hline & & $10-28-1960$ & 37.0 & & & & 03-06-1985 & 37.84 \\
\hline & & $12-12-1960$ & 36.9 & & & & $10-21-1985$ & 38.46 \\
\hline & & 03-31-1961 & 36.5 & & & & 03-04-1986 & 38.05 \\
\hline & & $10-16-1961$ & 37.0 & & & & 09-29-1986 & 28.70 \\
\hline
\end{tabular}


Table 2. Water levels in selected wells in Cedar Valley, Iron County, southwestern Utah-Continued

\begin{tabular}{|c|c|c|c|c|c|c|c|}
\hline Local well number & $\begin{array}{c}\text { Altitude of } \\
\text { land } \\
\text { surface } \\
\text { (feet) }\end{array}$ & Date & $\begin{array}{c}\text { Water level } \\
\text { (feet) }\end{array}$ & Local well number & $\begin{array}{l}\text { Altitude of } \\
\text { land } \\
\text { surface } \\
\text { (feet) }\end{array}$ & Date & $\begin{array}{l}\text { Water level } \\
\text { (feet) }\end{array}$ \\
\hline (C-33-11)30ddd-2-C & tinued & 03-12-1987 & 38.13 & $(\mathrm{C}-34-10) 31$ caa-1- & inued & $12-07-1959$ & 26.83 \\
\hline & & 09-17-1987 & 38.72 & & & 03-16-1960 & 24.31 \\
\hline & & 03-01-1988 & 38.10 & & & $10-28-1960$ & 29.81 \\
\hline & & 09-19-1988 & 38.75 & & & $12-06-1960$ & 27.90 \\
\hline & & 03-08-1989 & 36.56 & & & 04-04-1961 & 25.66 \\
\hline & & 09-22-1989 & 38.92 & & & 03-20-1962 & 26.30 \\
\hline & & 03-07-1990 & 39.43 & & & 03-25-1963 & 25.30 \\
\hline & & 09-22-1990 & 45.87 & & & 03-19-1964 & 26.44 \\
\hline & & 03-01-1991 & 42.25 & & & $11-02-1964$ & 31.95 \\
\hline & & 09-19-1991 & 39.01 & & & 03-17-1965 & 27.20 \\
\hline & & 03-09-1992 & 38.50 & & & 11-03-1965 & 30.40 \\
\hline & & 09-28-1992 & 38.91 & & & 03-10-1966 & 26.99 \\
\hline & & 03-17-1993 & 38.31 & & & 03-08-1967 & 27.97 \\
\hline & & 03-14-1994 & 38.55 & & & 03-12-1968 & 28.57 \\
\hline & & & & & & 03-17-1969 & 29.50 \\
\hline (C-33-11)31aad-1 & 5,355 & 09-10-1974 & 36.49 & & & 03-12-1970 & 29.20 \\
\hline & & 03-09-1995 & 37.58 & & & 03-08-1971 & 29.74 \\
\hline & & 03-07-1996 & 38.22 & & & 03-08-1972 & 30.06 \\
\hline & & 03-05-1997 & 37.72 & & & 03-15-1973 & 30.18 \\
\hline & & 11-24-1997 & 41.37 & & & 03-07-1974 & 28.01 \\
\hline & & 08-26-1998 & 40.60 & & & 03-11-1975 & 29.64 \\
\hline & & 11-17-1998 & 40.73 & & & 03-02-1976 & 30.83 \\
\hline & & 03-15-1999 & 40.08 & & & 03-01-1977 & 34.05 \\
\hline & & 11-01-1999 & 37.90 & & & $10-05-1977$ & 49.19 \\
\hline & & $03-13-2000$ & 37.33 & & & 03-02-1978 & 38.30 \\
\hline & & & & & & 03-16-1978 & 36.52 \\
\hline (C-33-11)31 aad-2 & 5,355 & 03-08-2001 & 38.79 & & & 03-06-1979 & 39.97 \\
\hline & & & & & & 03-14-1979 & 39.76 \\
\hline (C-33-11)33caa-1 & 5,369 & 08-28-1973 & 9.82 & & & 03-06-1980 & 39.30 \\
\hline & & 11-17-1998 & 18.50 & & & 03-03-1981 & 37.25 \\
\hline & & 03-15-1999 & 18.63 & & & 03-01-1982 & 37.07 \\
\hline & & 11-01-1999 & 13.78 & & & 03-07-1983 & 37.34 \\
\hline & & $03-13-2000$ & 13.86 & & & 03-08-1984 & 38.77 \\
\hline & & & & & & 03-05-1985 & 37.61 \\
\hline (C-34-10)31caa-1 & 5,490 & $12-07-1953$ & 21.42 & & & 03-04-1986 & 38.78 \\
\hline & & 12-03-1954 & 22.45 & & & 03-05-1987 & 37.99 \\
\hline & & 03-20-1955 & 20.19 & & & 03-01-1988 & 38.14 \\
\hline & & 10-11-1955 & 25.79 & & & 03-09-1989 & 38.25 \\
\hline & & 12-01-1955 & 23.51 & & & 03-07-1990 & 42.55 \\
\hline & & 03-14-1956 & 21.13 & & & & \\
\hline & & 10-18-1956 & 27.97 & (C-34-11)1daa-1 & 5,402 & $12-05-1960$ & 12.32 \\
\hline & & 12-13-1956 & 23.88 & & & 03-31-1961 & 12.00 \\
\hline & & 03-12-1957 & 21.61 & & & 03-22-1962 & 12.33 \\
\hline & & 10-24-1957 & 27.34 & & & $03-25-1963$ & 12.61 \\
\hline & & 12-03-1957 & 25.17 & & & 03-19-1964 & 13.42 \\
\hline & & 03-18-1958 & 22.33 & & & 11-02-1964 & 13.98 \\
\hline & & $10-25-1958$ & 27.88 & & & 03-17-1965 & 13.15 \\
\hline & & 12-16-1958 & 25.30 & & & 11-03-1965 & 14.06 \\
\hline & & 04-08-1959 & 22.83 & & & 03-10-1966 & 13.14 \\
\hline & & 10-13-1959 & 29.45 & & & 03-08-1967 & 14.58 \\
\hline & & $10-20-1959$ & 29.14 & & & 03-14-1969 & 14.44 \\
\hline
\end{tabular}


Table 2. Water levels in selected wells in Cedar Valley, Iron County, southwestern Utah—Continued

\begin{tabular}{|c|c|c|c|c|c|c|c|c|}
\hline Local well number & $\begin{array}{c}\text { Altitude of } \\
\text { land } \\
\text { surface } \\
\text { (feet) }\end{array}$ & Date & $\begin{array}{c}\text { Water level } \\
\text { (feet) }\end{array}$ & Local well number & $\begin{array}{c}\text { Altitude of } \\
\text { land } \\
\text { surface } \\
\text { (feet) }\end{array}$ & Date & \multicolumn{2}{|c|}{$\begin{array}{l}\text { Water level } \\
\text { (feet) }\end{array}$} \\
\hline \multirow{40}{*}{\multicolumn{2}{|c|}{ (C-34-11)1daa-1—Continued }} & $03-11-1970$ & 14.90 & \multicolumn{2}{|c|}{ (C-34-11)3ccd-1-Continued } & 11-01-1999 & 14.42 & \\
\hline & & 03-08-1971 & 15.37 & & & $03-13-2000$ & \multicolumn{2}{|l|}{13.76} \\
\hline & & 03-08-1972 & 15.83 & & & & & \\
\hline & & 03-15-1973 & 15.58 & (C-34-11)3dcb-1 & 5,390 & 08-29-1973 & 11.41 & \\
\hline & & 03-07-1974 & 16.08 & & & 03-18-1974 & 10.92 & \\
\hline & & 03-07-1975 & 17.95 & & & 08-26-1998 & 17.01 & \\
\hline & & 03-02-1976 & 19.20 & & & 11-17-1998 & 16.73 & \\
\hline & & 10-19-1976 & 22.65 & & & 03-15-1999 & 16.34 & \\
\hline & & 03-21-1977 & 19.91 & & & 11-01-1999 & 16.94 & \\
\hline & & $10-02-1977$ & 25.25 & & & 03-13-2000 & 15.03 & \\
\hline & & 03-02-1978 & 21.08 & & & & & \\
\hline & & 03-06-1978 & 20.98 & (C-34-11)5dcb-1 & 5,382 & 07-30-1973 & 30.18 & \\
\hline & & 03-06-1979 & 21.80 & & & 08-10-1998 & 32.77 & \\
\hline & & 10-03-1979 & 28.84 & & & 11-17-1998 & 32.77 & \\
\hline & & 03-06-1980 & 23.05 & & & 03-15-1999 & 32.39 & \\
\hline & & 03-03-1981 & 23.47 & & & 11-02-1999 & 33.02 & \\
\hline & & 03-01-1982 & 23.78 & & & 03-13-2000 & 32.52 & \\
\hline & & 03-01-1983 & 24.32 & & & & & \\
\hline & & 03-08-1984 & 24.26 & (C-34-11)9ccd-1 & 5,403 & $12-01-1970$ & 37 & $\mathrm{D}$ \\
\hline & & 03-06-1985 & 27.63 & & & 08-29-1973 & 32.61 & \\
\hline & & 03-03-1986 & 24.49 & & & 03-19-1974 & 32 & $\mathrm{R}$ \\
\hline & & 03-05-1987 & 24.50 & & & 08-10-1998 & 35.95 & \\
\hline & & 03-01-1988 & 24.31 & & & 11-17-1998 & 39.24 & \\
\hline & & 03-08-1989 & 22.88 & & & 03-15-1999 & 35.89 & \\
\hline & & 03-07-1990 & 23.04 & & & 11-02-1999 & 36.35 & \\
\hline & & 03-01-1991 & 24.15 & & & 03-13-2000 & 36.02 & \\
\hline & & 03-09-1992 & 23.18 & & & & & \\
\hline & & 03-05-1993 & 22.90 & (C-34-11)9cdc-1 & 5,402 & 06-17-1938 & 21.31 & \\
\hline & & 03-04-1994 & 23.30 & & & 07-13-1938 & 21.88 & \\
\hline & & 03-08-1995 & 23.35 & & & 08-01-1938 & 22.00 & \\
\hline & & 03-07-1996 & 22.65 & & & 08-30-1938 & 22.26 & \\
\hline & & 03-05-1997 & 22.60 & & & 09-28-1938 & 22.40 & \\
\hline & & 11-25-1997 & 22.90 & & & $11-02-1938$ & 22.25 & \\
\hline & & 03-16-1998 & 21.90 & & & $12-02-1938$ & 22.07 & \\
\hline & & 11-18-1998 & 23.70 & & & 01-04-1939 & 21.96 & \\
\hline & & 03-16-1999 & 22.16 & & & 02-02-1939 & 21.83 & \\
\hline & & 11-01-1999 & 24.80 & & & 04-04-1939 & 21.62 & \\
\hline & & 03-14-2000 & 22.72 & & & 05-09-1939 & 21.53 & \\
\hline & & 03-08-2001 & 24.54 & & & 06-12-1939 & 21.67 & \\
\hline & & & & & & 07-12-1939 & 22.15 & \\
\hline \multirow[t]{8}{*}{$(\mathrm{C}-34-11) 3 \mathrm{cca}-1$} & \multirow[t]{8}{*}{5,383} & 08-29-1973 & 6.89 & & & 08-17-1939 & 22.50 & \\
\hline & & 03-18-1974 & 6.27 & & & 09-14-1939 & 22.56 & \\
\hline & & 08-11-1998 & 12.78 & & & 12-11-1939 & 22.11 & \\
\hline & & 11-17-1998 & 12.32 & & & 04-01-1940 & 21.75 & \\
\hline & & 03-15-1999 & 11.73 & & & 05-01-1940 & 21.67 & \\
\hline & & 11-01-1999 & 12.66 & & & 06-13-1940 & 21.82 & \\
\hline & & $03-13-2000$ & 11.85 & & & $12-02-1940$ & 22.33 & \\
\hline & & & & & & 03-24-1941 & 22.91 & \\
\hline \multirow[t]{3}{*}{ (C-34-11)3ccd-1 } & \multirow[t]{3}{*}{5,385} & 08-11-1998 & 14.27 & & & 12-01-1941 & 22.38 & \\
\hline & & 11-17-1998 & 14.13 & & & 03-14-1942 & 22.01 & \\
\hline & & 03-15-1999 & 13.74 & & & 08-06-1942 & 22.55 & \\
\hline
\end{tabular}


Table 2. Water levels in selected wells in Cedar Valley, Iron County, southwestern Utah-Continued

\begin{tabular}{|c|c|c|c|c|c|c|c|}
\hline Local well number & $\begin{array}{l}\text { Altitude of } \\
\text { land } \\
\text { surface } \\
\text { (feet) }\end{array}$ & Date & $\begin{array}{c}\text { Water level } \\
\text { (feet) }\end{array}$ & Local well number & $\begin{array}{l}\text { Altitude of } \\
\text { land } \\
\text { surface } \\
\text { (feet) }\end{array}$ & Date & $\begin{array}{l}\text { Water level } \\
\text { (feet) }\end{array}$ \\
\hline \multirow{51}{*}{\multicolumn{2}{|c|}{ (C-34-11)9cdc-1—Continued }} & $12-16-1942$ & 22.40 & \multirow{51}{*}{\multicolumn{2}{|c|}{ (C-34-11)9cdc-1—Continued }} & 03-14-1969 & 22.47 \\
\hline & & $12-11-1943$ & 22.43 & & & $10-08-1969$ & 23.25 \\
\hline & & $12-07-1944$ & 22.17 & & & 03-11-1970 & 22.72 \\
\hline & & 03-30-1945 & 21.60 & & & $10-08-1970$ & 23.19 \\
\hline & & 12-09-1945 & 22.13 & & & 03-08-1971 & 22.70 \\
\hline & & 03-18-1946 & 21.77 & & & 09-30-1971 & 23.34 \\
\hline & & 12-11-1946 & 21.87 & & & 03-08-1972 & 22.82 \\
\hline & & 03-22-1947 & 21.56 & & & 10-04-1972 & 23.42 \\
\hline & & $12-05-1948$ & 22.26 & & & $10-04-1973$ & 23.50 \\
\hline & & 04-18-1949 & 21.85 & & & 03-07-1974 & 22.88 \\
\hline & & 12-07-1949 & 22.22 & & & $10-22-1974$ & 23.13 \\
\hline & & 03-22-1950 & 21.78 & & & 03-07-1975 & 22.79 \\
\hline & & $12-06-1950$ & 22.30 & & & $10-08-1975$ & 23.29 \\
\hline & & 03-20-1951 & 21.94 & & & 03-02-1976 & 22.93 \\
\hline & & 04-02-1952 & 21.99 & & & $10-19-1976$ & 23.45 \\
\hline & & $12-03-1952$ & 22.56 & & & 03-01-1977 & 23.00 \\
\hline & & $03-12-1953$ & 22.19 & & & $10-03-1977$ & 23.61 \\
\hline & & $12-07-1953$ & 22.22 & & & 03-16-1978 & 23.57 \\
\hline & & $12-03-1954$ & 22.49 & & & $10-02-1978$ & 23.73 \\
\hline & & 03-18-1955 & 22.13 & & & 03-13-1979 & 22.60 \\
\hline & & $12-01-1955$ & 22.21 & & & 10-02-1979 & 23.18 \\
\hline & & 03-14-1956 & 21.91 & & & 03-06-1980 & 23.02 \\
\hline & & $10-22-1956$ & 22.60 & & & $10-01-1980$ & 23.46 \\
\hline & & $12-13-1956$ & 22.36 & & & 03-03-1981 & 23.20 \\
\hline & & 03-12-1957 & 22.06 & & & $10-01-1981$ & 23.59 \\
\hline & & $10-24-1957$ & 22.70 & & & 03-01-1982 & 23.33 \\
\hline & & $12-03-1957$ & 22.43 & & & $10-01-1982$ & 23.90 \\
\hline & & 03-18-1958 & 22.12 & & & 03-01-1983 & 23.46 \\
\hline & & $10-27-1958$ & 23.30 & & & $10-12-1983$ & 23.94 \\
\hline & & $12-16-1958$ & 23.27 & & & 03-08-1984 & 23.60 \\
\hline & & 04-08-1959 & 22.77 & & & 09-26-1984 & 24.05 \\
\hline & & $10-26-1959$ & 23.10 & & & 03-06-1985 & 25.64 \\
\hline & & 12-05-1959 & 22.84 & & & $10-21-1985$ & 25.92 \\
\hline & & 03-16-1960 & 22.37 & & & 03-04-1986 & 25.48 \\
\hline & & $10-28-1960$ & 22.82 & & & $03-12-1987$ & 24.74 \\
\hline & & $12-12-1960$ & 22.58 & & & $09-17-1987$ & 24.99 \\
\hline & & 03-31-1961 & 22.23 & & & 03-01-1988 & 24.70 \\
\hline & & $10-16-1961$ & 22.77 & & & 09-19-1988 & 24.24 \\
\hline & & 03-27-1962 & 22.18 & & & 03-08-1989 & 24.84 \\
\hline & & $10-10-1962$ & 22.99 & & & 09-22-1989 & 24.53 \\
\hline & & 03-25-1963 & 22.72 & & & 03-07-1990 & 24.10 \\
\hline & & $10-28-1963$ & 23.01 & & & $09-22-1990$ & 24.66 \\
\hline & & 03-19-1964 & 22.45 & & & 03-01-1991 & 24.14 \\
\hline & & $11-02-1964$ & 23.02 & & & 09-19-1991 & 26.66 \\
\hline & & $03-17-1965$ & 22.45 & & & 09-28-1992 & 26.58 \\
\hline & & $11-03-1965$ & 23.00 & & & 03-02-1993 & 26.02 \\
\hline & & 03-10-1966 & 22.44 & & & 03-04-1994 & 25.88 \\
\hline & & 09-27-1966 & 23.14 & & & 03-09-1995 & 25.37 \\
\hline & & 03-08-1967 & 22.55 & & & 03-07-1996 & 26.02 \\
\hline & & $10-11-1967$ & 22.92 & & & 03-05-1997 & 24.75 \\
\hline & & $10-08-1968$ & 22.90 & & & $11-24-1997$ & 25.09 \\
\hline
\end{tabular}


Table 2. Water levels in selected wells in Cedar Valley, Iron County, southwestern Utah—Continued

\begin{tabular}{|c|c|c|c|c|}
\hline Local well number & $\begin{array}{l}\text { Altitude of } \\
\text { land } \\
\text { surface } \\
\text { (feet) }\end{array}$ & Date & $\begin{array}{l}\text { Water leve } \\
\text { (feet) }\end{array}$ & \\
\hline \multicolumn{2}{|c|}{ (C-34-11)9cdc-1-Continued } & 03-16-1998 & 24.70 & \\
\hline & & 11-17-1998 & 25.08 & \\
\hline & & 03-15-1999 & 24.67 & \\
\hline & & 11-02-1999 & 25.30 & \\
\hline & & 03-13-2000 & 24.76 & \\
\hline & & 03-08-2001 & 24.67 & \\
\hline \multirow[t]{4}{*}{ (C-34-11)11dac-1 } & 5,387 & 08-27-1998 & 13.16 & \\
\hline & & 03-16-1999 & 7.88 & \\
\hline & & 11-01-1999 & 10.90 & \\
\hline & & 03-14-2000 & 8.39 & \\
\hline \multirow[t]{5}{*}{ (C-34-11)13bcd-1 } & 5,400 & 08-27-1998 & 32.29 & \\
\hline & & 11-17-1998 & 28.24 & \\
\hline & & 03-16-1999 & 26.40 & \\
\hline & & 11-01-1999 & 29.48 & \\
\hline & & 03-14-2000 & 26.99 & \\
\hline \multirow[t]{6}{*}{ (C-34-11)14aad-2 } & 5,395 & 03-08-1984 & 20.29 & \\
\hline & & 03-06-1985 & 19.19 & \\
\hline & & 11-18-1998 & 18.50 & \\
\hline & & 03-16-1999 & 16.69 & \\
\hline & & 11-01-1999 & 19.51 & \\
\hline & & 03-14-2000 & 17.14 & \\
\hline \multirow[t]{6}{*}{ (C-34-11)16bcc-1 } & 5,408 & 03-19-1974 & 21.80 & \\
\hline & & 08-10-1998 & 22.75 & \\
\hline & & 11-17-1998 & 22.69 & \\
\hline & & 03-15-1999 & 22.54 & \\
\hline & & 11-02-1999 & 23.64 & \\
\hline & & $03-13-2000$ & 23.09 & \\
\hline \multirow[t]{8}{*}{ (C-34-11)20dba-1 } & 5,430 & $10-13-1955$ & 26 & $\mathrm{D}$ \\
\hline & & 08-29-1973 & 42.69 & \\
\hline & & 03-19-1974 & 30 & $\mathrm{R}$ \\
\hline & & 08-10-1998 & 30.69 & \\
\hline & & 11-17-1998 & 30.68 & \\
\hline & & 03-15-1999 & 30.65 & \\
\hline & & 11-02-1999 & 30.90 & \\
\hline & & 03-13-2000 & 30.90 & \\
\hline \multirow[t]{7}{*}{ (C-34-11)21dcd-1 } & 5,425 & 06-15-1946 & 20 & $\mathrm{D}$ \\
\hline & & 08-30-1973 & 23.98 & \\
\hline & & 03-19-1974 & 5 & $\mathrm{R}$ \\
\hline & & 11-16-1998 & 25.15 & \\
\hline & & 03-15-1999 & 36.37 & \\
\hline & & 11-01-1999 & 31.02 & \\
\hline & & $03-13-2000$ & 23.28 & \\
\hline
\end{tabular}

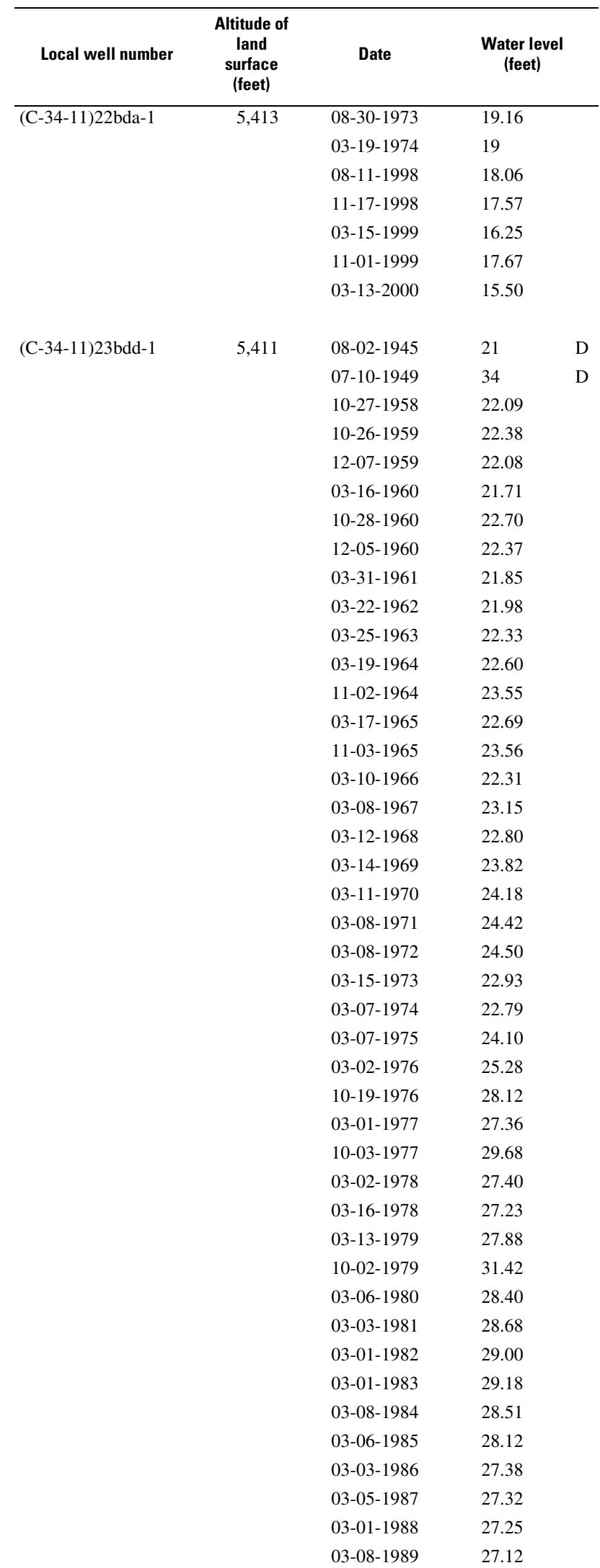


Table 2. Water levels in selected wells in Cedar Valley, Iron County, southwestern Utah-Continued

\begin{tabular}{|c|c|c|c|c|c|c|c|c|c|}
\hline Local well number & $\begin{array}{c}\text { Altitude of } \\
\text { land } \\
\text { surface } \\
\text { (feet) }\end{array}$ & Date & $\begin{array}{c}\text { Water level } \\
\text { (feet) }\end{array}$ & & Local well number & $\begin{array}{c}\text { Altitude of } \\
\text { land } \\
\text { surface } \\
\text { (feet) }\end{array}$ & Date & $\begin{array}{c}\text { Water level } \\
\text { (feet) }\end{array}$ & \\
\hline$(\mathrm{C}-34-11) 23$ bdd-1-C & tinued & 03-07-1990 & 28.38 & & $(\mathrm{C}-34-11) 36 \mathrm{dbb}-1-\mathrm{C}$ & tinued & 11-16-1998 & 55.54 & \\
\hline & & 03-01-1991 & 27.83 & & & & 03-16-1999 & 38.13 & \\
\hline & & 03-09-1992 & 28.10 & & & & 11-02-1999 & 60.55 & \\
\hline & & 03-05-1993 & 28.50 & & & & 03-14-2000 & 39.41 & \\
\hline & & 03-04-1994 & 28.57 & & & & & & \\
\hline & & 03-08-1995 & 28.72 & & (C-34-11)36dcc-2 & 5,460 & 04-03-1952 & 1.5 & $\mathrm{D}$ \\
\hline & & 03-07-1996 & 28.44 & & & & 03-18-1955 & 6.92 & \\
\hline & & 03-05-1997 & 28.53 & & & & $12-01-1955$ & 11.37 & \\
\hline & & 11-25-1997 & 29.13 & & & & 03-14-1956 & 9.43 & \\
\hline & & 03-16-1998 & 28.12 & & & & $10-18-1956$ & 43.83 & \\
\hline & & 11-18-1998 & 29.13 & & & & $12-13-1956$ & 10.52 & \\
\hline & & 03-16-1999 & 28.07 & & & & $10-24-1957$ & 42.51 & \\
\hline & & 11-01-1999 & 30.02 & & & & $12-03-1957$ & 25.84 & \\
\hline & & 03-14-2000 & 28.53 & & & & 03-18-1958 & 13.00 & \\
\hline & & 03-08-2001 & 29.26 & & & & $10-27-1958$ & 52.61 & \\
\hline & & & & & & & $12-16-1958$ & 24.19 & \\
\hline (C-34-11)29ddd-1 & 5,542 & 04-03-1940 & 16 & $\mathrm{R}$ & & & 04-08-1959 & 13.99 & \\
\hline & & 11-16-1998 & 20.96 & & & & 10-26-1959 & 41.49 & \\
\hline & & 03-16-1999 & 19.73 & & & & $12-07-1959$ & 26.78 & \\
\hline & & 11-02-1999 & 21.57 & & & & 03-16-1960 & 15.12 & \\
\hline & & 03-13-2000 & 19.85 & & & & $10-28-1960$ & 44.57 & \\
\hline & & & & & & & $12-05-1960$ & 28.33 & \\
\hline (C-34-11)31cca-1 & 5,580 & 03-17-1999 & 215.75 & & & & 03-31-1961 & 16.09 & \\
\hline & & 02-03-2000 & 216.00 & & & & 03-22-1962 & 12.37 & \\
\hline & & $03-15-2000$ & 215.82 & & & & 03-25-1963 & 14.20 & \\
\hline & & 03-08-2001 & 216.28 & & & & 03-19-1964 & 15.23 & \\
\hline & & & & & & & 11-02-1964 & 25.03 & \\
\hline (C-34-11)36dbb-1 & 5,460 & 04-30-1957 & 20 & D & & & 03-17-1965 & 15.67 & \\
\hline & & 10-04-1977 & 67.58 & & & & 11-03-1965 & 17.15 & \\
\hline & & 03-02-1978 & 37.14 & & & & 03-10-1966 & 14.70 & \\
\hline & & 03-16-1978 & 36.28 & & & & 03-08-1967 & 14.82 & \\
\hline & & 03-13-1979 & 35.20 & & & & 03-12-1968 & 14.78 & \\
\hline & & 03-03-1981 & 33.76 & & & & 03-14-1969 & 15.85 & \\
\hline & & 03-01-1982 & 31.68 & & & & 03-11-1970 & 16.07 & \\
\hline & & 03-01-1983 & 33.38 & & & & 03-08-1971 & 17.17 & \\
\hline & & 03-07-1984 & 32.17 & & & & 03-08-1972 & 16.84 & \\
\hline & & 03-06-1985 & 30.31 & & & & 03-15-1973 & 16.22 & \\
\hline & & 03-03-1986 & 28.90 & & & & 03-07-1974 & 13.60 & \\
\hline & & 03-05-1987 & 29.10 & & & & 03-07-1975 & 15.81 & \\
\hline & & 03-01-1988 & 29.95 & & & & 03-02-1976 & 16.95 & \\
\hline & & 03-08-1989 & 27.90 & & & & 03-01-1977 & 26.19 & \\
\hline & & 03-07-1990 & 31.30 & & & & 03-11-1977 & 25.58 & \\
\hline & & 03-01-1991 & 32.81 & & & & 10-05-1977 & 24.35 & \\
\hline & & 03-09-1992 & 35.12 & & & & 03-02-1978 & 51.92 & \\
\hline & & 03-05-1993 & 37.00 & & & & 03-06-1978 & 31.66 & \\
\hline & & 03-09-1994 & 37.38 & & & & 03-16-1978 & 30.73 & \\
\hline & & 03-08-1995 & 42.30 & & & & 03-13-1979 & 30.37 & \\
\hline & & 03-07-1996 & 37.94 & & & & 03-06-1980 & 32.31 & \\
\hline & & 03-05-1997 & 40.97 & & & & 03-03-1981 & 30.16 & \\
\hline & & 11-25-1997 & 52.50 & & & & 03-01-1982 & 27.29 & \\
\hline & & 03-16-1998 & 38.80 & & & & 03-01-1983 & 28.82 & \\
\hline
\end{tabular}


Table 2. Water levels in selected wells in Cedar Valley, Iron County, southwestern Utah-Continued

\begin{tabular}{|c|c|c|c|c|c|c|c|c|}
\hline Local well number & $\begin{array}{l}\text { Altitude of } \\
\text { land } \\
\text { surface } \\
\text { (feet) }\end{array}$ & Date & $\begin{array}{l}\text { Water level } \\
\text { (feet) }\end{array}$ & Local well number & $\begin{array}{l}\text { Altitude of } \\
\text { land } \\
\text { surface } \\
\text { (feet) }\end{array}$ & Date & \multicolumn{2}{|c|}{$\begin{array}{l}\text { Water level } \\
\text { (feet) }\end{array}$} \\
\hline \multirow[t]{22}{*}{$(\mathrm{C}-34-11) 36 \mathrm{dcc}-2-\mathrm{C}$} & tinued & 03-08-1984 & 28.68 & $(\mathrm{C}-35-10) 7 \mathrm{abc}-1-\mathrm{Co}$ & nued & $11-25-1997$ & 118.40 & \\
\hline & & 03-06-1985 & 30.71 & & & 03-13-1998 & 116.93 & \\
\hline & & 03-03-1986 & 25.26 & & & 11-18-1998 & 116.99 & \\
\hline & & 03-05-1987 & 25.84 & & & 03-16-1999 & 114.11 & \\
\hline & & 03-01-1988 & 24.28 & & & 11-02-1999 & 117.00 & \\
\hline & & 03-08-1989 & 26.32 & & & $03-14-2000$ & 113.47 & \\
\hline & & 03-07-1990 & 27.22 & & & 03-08-2001 & 117.35 & \\
\hline & & 03-01-1991 & 28.78 & & & & & \\
\hline & & 03-09-1992 & 31.30 & $(\mathrm{C}-35-10) 7 \mathrm{bcd}-1$ & 5,510 & $11-18-1998$ & 59.33 & \\
\hline & & 03-05-1993 & 33.38 & & & 03-16-1999 & 49.48 & \\
\hline & & 03-09-1994 & 33.78 & & & 11-02-1999 & 62.60 & \\
\hline & & 03-08-1995 & 36.53 & & & $03-15-2000$ & 51.74 & \\
\hline & & 03-07-1996 & 35.29 & & & & & \\
\hline & & 03-05-1997 & 37.96 & (C-35-10) $18 \mathrm{bbc}-1$ & 5,536 & $10-25-1973$ & 78.31 & \\
\hline & & $11-25-1997$ & 51.79 & & & 03-19-1974 & 71 & $\mathrm{R}$ \\
\hline & & 03-16-1998 & 35.80 & & & 08-17-1998 & 97.97 & \\
\hline & & $11-16-1998$ & 57.08 & & & $11-18-1998$ & 86.55 & \\
\hline & & 03-16-1999 & 36.34 & & & 03-16-1999 & 75.89 & \\
\hline & & 11-02-1999 & 64.97 & & & 11-02-1999 & 89.95 & \\
\hline & & $03-14-2000$ & 36.68 & & & 03-14-2000 & 78.89 & \\
\hline & & 03-08-2001 & 42.21 & & & & & \\
\hline & & & & (C-35-10) 18bcb-1 & 5,540 & 08-17-1998 & 94.84 & \\
\hline \multirow[t]{4}{*}{ (C-34-11)36dcc-3 } & 5,465 & $11-16-1998$ & 60.69 & & & $11-18-1998$ & 84.06 & \\
\hline & & 03-16-1999 & 40.22 & & & 03-16-1999 & 75.29 & \\
\hline & & 11-02-1999 & 68.23 & & & 11-02-1999 & 88.43 & \\
\hline & & $03-14-2000$ & 40.71 & & & $03-14-2000$ & 78.18 & \\
\hline \multirow[t]{24}{*}{$(\mathrm{C}-35-10) 7 \mathrm{abc}-1$} & 5,570 & $06-25-1966$ & 58 & $(\mathrm{C}-35-11) 1 \mathrm{bdc}-1$ & 5,460 & $11-18-1998$ & 59.14 & \\
\hline & & 10-04-1977 & 125.54 & & & 03-16-1999 & 37.02 & \\
\hline & & 03-02-1978 & 109.34 & & & 11-03-1999 & 67.00 & \\
\hline & & 03-20-1978 & 108.79 & & & 03-14-2000 & 37.73 & \\
\hline & & 03-06-1979 & 109.36 & & & & & \\
\hline & & 03-14-1979 & 109.03 & (C-35-11)4aba-1 & 5,456 & 03-22-1950 & 9.92 & \\
\hline & & 03-06-1980 & 111.17 & & & $12-06-1950$ & 19.00 & \\
\hline & & 03-02-1981 & 108.99 & & & 03-20-1951 & 18.85 & \\
\hline & & 03-01-1982 & 109.07 & & & 04-02-1952 & 19.33 & \\
\hline & & 03-01-1983 & 109.46 & & & 12-03-1952 & 19.87 & \\
\hline & & 03-08-1984 & 108.60 & & & $03-12-1953$ & 18.97 & \\
\hline & & 03-05-1985 & 106.24 & & & $12-07-1953$ & 19.90 & \\
\hline & & 03-03-1986 & 105.07 & & & 03-18-1955 & 19.20 & \\
\hline & & 03-05-1987 & 103.05 & & & 12-01-1955 & 19.62 & \\
\hline & & 03-01-1988 & 102.09 & & & 03-14-1956 & 19.74 & \\
\hline & & 03-09-1989 & 102.23 & & & $10-23-1956$ & 20.87 & \\
\hline & & 03-07-1990 & 103.13 & & & $12-13-1956$ & 20.58 & \\
\hline & & 03-01-1991 & 106.18 & & & 03-12-1957 & 20.12 & \\
\hline & & 03-09-1992 & 108.27 & & & $10-24-1957$ & 21.11 & \\
\hline & & 03-05-1993 & 110.32 & & & 12-03-1957 & 20.71 & \\
\hline & & 03-09-1994 & 110.12 & & & 03-18-1958 & 20.24 & \\
\hline & & 03-08-1995 & 111.93 & & & $10-27-1958$ & 21.13 & \\
\hline & & 03-07-1996 & 113.82 & & & $12-16-1958$ & 20.53 & \\
\hline & & 03-04-1997 & 115.85 & & & 04-08-1959 & 19.60 & \\
\hline
\end{tabular}


Table 2. Water levels in selected wells in Cedar Valley, Iron County, southwestern Utah-Continued

\begin{tabular}{|c|c|c|c|c|c|c|c|c|c|}
\hline Local well number & $\begin{array}{l}\text { Altitude of } \\
\text { land } \\
\text { surface } \\
\text { (feet) }\end{array}$ & Date & $\begin{array}{c}\text { Water level } \\
\text { (feet) }\end{array}$ & & Local well number & $\begin{array}{l}\text { Altitude of } \\
\text { land } \\
\text { surface } \\
\text { (feet) }\end{array}$ & Date & $\begin{array}{l}\text { Water level } \\
\text { (feet) }\end{array}$ & \\
\hline (C-35-11)4aba-1-Con & inued & $10-26-1959$ & 20.88 & & $\overline{(\mathrm{C}-35-11) 4 a b a-1-\mathrm{Co}}$ & nued & 03-16-1998 & 14.58 & \\
\hline & & $12-05-1959$ & 20.28 & & & & $11-16-1998$ & 16.64 & \\
\hline & & $03-16-1960$ & 19.78 & & & & 03-16-1999 & 12.85 & \\
\hline & & $10-28-1960$ & 20.86 & & & & 11-03-1999 & 19.34 & \\
\hline & & $12-12-1960$ & 20.43 & & & & 03-14-2000 & 13.63 & \\
\hline & & 03-31-1961 & 19.95 & & & & 03-08-2001 & 15.59 & \\
\hline & & $03-27-1962$ & 20.06 & & & & & & \\
\hline & & $03-25-1963$ & 20.27 & & (C-35-11)4aba-2 & 5,456 & $11-24-1997$ & 19.36 & \\
\hline & & 03-19-1964 & 20.70 & & & & 03-16-1998 & 18.20 & \\
\hline & & $11-02-1964$ & 21.54 & & & & $11-17-1998$ & 18.50 & \\
\hline & & $03-17-1965$ & 20.57 & & & & 03-16-1999 & 17.21 & \\
\hline & & $11-03-1965$ & 21.41 & & & & 11-03-1999 & 18.99 & \\
\hline & & 03-10-1966 & 20.57 & & & & 03-14-2000 & 17.47 & \\
\hline & & 03-06-1967 & 20.50 & & & & & & \\
\hline & & 03-14-1969 & 23.15 & & $(\mathrm{C}-35-11) 5 \mathrm{bbc}-1$ & 5,464 & $11-10-1942$ & 23 & $\mathrm{D}$ \\
\hline & & 03-11-1970 & 22.83 & & & & 10-03-1977 & 30.06 & \\
\hline & & 03-08-1971 & 20.56 & & & & 03-06-1978 & 29.45 & \\
\hline & & 03-08-1972 & 24.33 & & & & 03-16-1978 & 29.44 & \\
\hline & & 03-15-1973 & 19.12 & & & & $10-03-1978$ & 30.20 & \\
\hline & & 03-07-1974 & 22.36 & & & & 03-08-1979 & 29.10 & \\
\hline & & 03-22-1974 & & $\mathrm{R}$ & & & $10-02-1979$ & 30.09 & \\
\hline & & 03-07-1975 & 23.06 & & & & 03-03-1981 & 29.13 & \\
\hline & & 03-02-1976 & 23.07 & & & & 03-01-1982 & 29.02 & \\
\hline & & $10-19-1976$ & 21.39 & & & & 03-01-1983 & 28.80 & \\
\hline & & 03-01-1977 & 20.69 & & & & 03-08-1984 & 28.11 & \\
\hline & & 03-11-1977 & 20.69 & & & & 03-05-1985 & 27.60 & \\
\hline & & 10-04-1977 & 25.65 & & & & 03-03-1986 & 27.10 & \\
\hline & & 03-01-1978 & 19.49 & & & & 03-12-1987 & 27.27 & \\
\hline & & 03-06-1978 & 19.42 & & & & 03-01-1988 & 26.32 & \\
\hline & & 03-16-1978 & 19.52 & & & & 03-08-1989 & 26.35 & \\
\hline & & 03-05-1979 & 19.19 & & & & 03-07-1990 & 27.19 & \\
\hline & & $10-01-1979$ & 23.76 & & & & 03-01-1991 & 26.34 & \\
\hline & & 03-06-1980 & 17.21 & & & & 03-09-1992 & 26.73 & \\
\hline & & 03-03-1981 & 15.67 & & & & 03-02-1993 & 27.19 & \\
\hline & & 03-01-1982 & 15.65 & & & & 03-04-1994 & 27.24 & \\
\hline & & 03-01-1983 & 14.95 & & & & 03-09-1995 & 27.50 & \\
\hline & & 03-08-1984 & 12.30 & & & & 03-07-1996 & 27.68 & \\
\hline & & 03-05-1985 & 10.78 & & & & 03-04-1997 & 28.06 & \\
\hline & & 03-03-1986 & 10.30 & & & & $11-24-1997$ & 28.73 & \\
\hline & & 03-05-1987 & 10.40 & & & & 03-16-1998 & 28.17 & \\
\hline & & 03-01-1988 & 9.72 & & & & & & \\
\hline & & 03-09-1989 & 10.05 & & (C-35-11)8ddc-1 & 5,492 & 04-30-1953 & 21 & $\mathrm{D}$ \\
\hline & & 03-07-1990 & 11.94 & & & & 08-18-1998 & 36.10 & \\
\hline & & 03-01-1991 & 13.81 & & & & 11-19-1998 & 13.30 & \\
\hline & & 03-09-1992 & 15.27 & & & & 03-16-1999 & 8.27 & \\
\hline & & 03-02-1993 & 15.56 & & & & 11-03-1999 & 21.32 & \\
\hline & & 03-09-1994 & 14.25 & & & & 03-14-2000 & 18.05 & \\
\hline & & 03-08-1995 & 15.05 & & & & & & \\
\hline & & 03-07-1996 & 13.00 & & (C-35-11)9abc-1 & 5,477 & $10-26-1973$ & 14.27 & \\
\hline & & 03-04-1997 & 14.79 & & & & 03-20-1974 & 15 & $\mathrm{R}$ \\
\hline & & $11-24-1997$ & 17.86 & & & & 08-11-1998 & 13.63 & \\
\hline
\end{tabular}


Table 2. Water levels in selected wells in Cedar Valley, Iron County, southwestern Utah-Continued

\begin{tabular}{|c|c|c|c|c|c|c|c|c|c|}
\hline Local well number & $\begin{array}{l}\text { Altitude of } \\
\text { land } \\
\text { surface } \\
\text { (feet) }\end{array}$ & Date & $\begin{array}{c}\text { Water level } \\
\text { (feet) }\end{array}$ & & Local well number & $\begin{array}{l}\text { Altitude of } \\
\text { land } \\
\text { surface } \\
\text { (feet) }\end{array}$ & Date & $\begin{array}{c}\text { Water level } \\
\text { (feet) }\end{array}$ & \\
\hline \multicolumn{2}{|c|}{ (C-35-11)9abc-1-Continued } & 11-16-1998 & 13.72 & & \multicolumn{2}{|c|}{ (C-35-11)12ccc-1-Continued } & $11-18-1998$ & \multicolumn{2}{|l|}{23.70} \\
\hline & & 03-16-1999 & 13.66 & & & & 03-16-1999 & 20.57 & \\
\hline & & 11-03-1999 & 14.88 & & & & 11-02-1999 & 25.09 & \\
\hline & & 03-14-2000 & 14.85 & & & & $03-14-2000$ & 21.40 & \\
\hline \multirow[t]{30}{*}{ (C-35-11)11ccc-1 } & \multirow[t]{30}{*}{5,495} & $10-04-1977$ & 54.90 & & (C-35-11)12dcd-1 & 5,498 & $10-24-1973$ & 33.67 & \\
\hline & & 03-02-1978 & 26.45 & & & & 03-19-1974 & 19 & $\mathrm{R}$ \\
\hline & & 03-16-1978 & 26.13 & & & & $10-04-1977$ & 50.31 & \\
\hline & & 03-16-1979 & 23.92 & & & & 03-02-1978 & 34.33 & \\
\hline & & 03-07-1980 & 20.52 & & & & 03-16-1978 & 33.63 & \\
\hline & & 03-02-1981 & 16.26 & & & & 03-06-1979 & 32.48 & \\
\hline & & 03-01-1982 & 15.50 & & & & 03-07-1980 & 31.72 & \\
\hline & & 03-01-1983 & 14.35 & & & & 03-02-1981 & 16.71 & \\
\hline & & 03-07-1984 & 9.00 & & & & 03-01-1982 & 27.02 & \\
\hline & & 03-05-1985 & 7.16 & & & & 03-01-1983 & 28.09 & \\
\hline & & 03-03-1986 & 7.53 & & & & 03-07-1984 & 25.34 & \\
\hline & & 03-05-1987 & 7.71 & & & & 03-05-1985 & 22.52 & \\
\hline & & 03-01-1988 & 8.14 & & & & 03-03-1986 & 22.87 & \\
\hline & & 03-09-1989 & 6.50 & & & & 03-05-1987 & 19.65 & \\
\hline & & 03-07-1990 & 12.72 & & & & 03-01-1988 & 18.28 & \\
\hline & & 03-01-1991 & 16.09 & & & & 03-09-1989 & 17.79 & \\
\hline & & 03-09-1992 & 19.70 & & & & 03-07-1990 & 22.30 & \\
\hline & & 03-02-1993 & 20.80 & & & & 03-01-1991 & 25.18 & \\
\hline & & 03-09-1994 & 18.62 & & & & 03-09-1992 & 28.35 & \\
\hline & & 03-08-1995 & 22.35 & & & & 03-02-1993 & 31.72 & \\
\hline & & 03-11-1996 & 17.54 & & & & 03-09-1994 & 28.97 & \\
\hline & & 03-04-1997 & 22.65 & & & & 03-08-1995 & 31.87 & \\
\hline & & $11-25-1997$ & 28.20 & & & & 03-07-1996 & 28.97 & \\
\hline & & 03-16-1998 & 23.89 & & & & 03-04-1997 & 33.64 & \\
\hline & & 11-18-1998 & 27.38 & & & & $11-25-1997$ & 40.28 & \\
\hline & & 03-16-1999 & 20.76 & & & & 03-16-1998 & 33.74 & \\
\hline & & 11-03-1999 & 32.07 & & & & 11-18-1998 & 40.20 & \\
\hline & & 03-14-2000 & 22.24 & & & & 03-16-1999 & 32.22 & \\
\hline & & 03-08-2001 & 27.44 & & & & 11-02-1999 & 43.52 & \\
\hline & & & & & & & 03-14-2000 & 34.38 & \\
\hline \multirow[t]{7}{*}{ (C-35-11)11ccd-1 } & \multirow[t]{7}{*}{5,493} & $10-24-1973$ & 21.06 & & & & 03-08-2001 & 38.12 & \\
\hline & & 03-19-1974 & 13 & $\mathrm{R}$ & & & & & \\
\hline & & 08-17-1998 & 62.46 & & (C-35-11)12ddd-2 & 5,514 & 08-17-1998 & 79.64 & \\
\hline & & 11-18-1998 & 27.68 & & & & 11-18-1998 & 64.59 & \\
\hline & & 03-16-1999 & 22.13 & & & & 03-16-1999 & 54.59 & \\
\hline & & 11-02-1999 & 31.51 & & & & 11-02-1999 & 68.06 & \\
\hline & & 03-14-2000 & 23.22 & & & & 03-14-2000 & 57.41 & \\
\hline \multirow[t]{5}{*}{ (C-35-11)12add-1 } & \multirow[t]{5}{*}{5,490} & 11-18-1998 & 39.25 & & (C-35-11)13ddb-1 & 5,542 & 03-17-1999 & 77.65 & \\
\hline & & 03-23-1999 & 29.59 & & & & 11-01-1999 & 89.27 & \\
\hline & & 11-03-1999 & 42.30 & & & & 03-14-2000 & 80.72 & \\
\hline & & 03-14-2000 & 31.62 & & & & & & \\
\hline & & & & & (C-35-11)14bac-1 & 5,495 & 09-15-1976 & 35 & $\mathrm{D}$ \\
\hline \multirow[t]{3}{*}{ (C-35-11) $12 \mathrm{ccc}-1$} & \multirow[t]{3}{*}{5,488} & $10-24-1973$ & 21 & $\mathrm{R}$ & & & 08-18-1998 & 62.27 & \\
\hline & & 03-19-1974 & 8 & $\mathrm{R}$ & & & 11-18-1998 & 29.13 & \\
\hline & & 08-17-1998 & 28.55 & & & & 03-17-1999 & 23.34 & \\
\hline
\end{tabular}


Table 2. Water levels in selected wells in Cedar Valley, Iron County, southwestern Utah-Continued

\begin{tabular}{|c|c|c|c|c|c|c|}
\hline Local well number & $\begin{array}{c}\text { Altitude of } \\
\text { land } \\
\text { surface } \\
\text { (feet) }\end{array}$ & Date & $\begin{array}{c}\text { Water level } \\
\text { (feet) }\end{array}$ & $\begin{array}{l}\text { Altitude of } \\
\text { land } \\
\text { surface } \\
\text { (feet) }\end{array}$ & Date & $\begin{array}{l}\text { Water level } \\
\text { (feet) }\end{array}$ \\
\hline$(\mathrm{C}-35-11) 14 \mathrm{bac}-1-\mathrm{C}$ & tinued & 11-01-1999 & 23.46 & (C-35-11)17dcd-1-Continued & 05-08-1939 & 16.88 \\
\hline & & $03-14-2000$ & 24.68 & & 06-12-1939 & 18.43 \\
\hline & & 03-08-2001 & 29.15 & & 07-11-1939 & 19.20 \\
\hline & & $05-24-2001$ & 46.31 & & 08-16-1939 & 19.91 \\
\hline & & & & & 09-08-1939 & 19.85 \\
\hline (C-35-11)14bac-2 & $5,494.5$ & 03-08-2001 & 26.21 & & 09-13-1939 & 19.80 \\
\hline & & 03-13-2001 & 26.15 & & 10-21-1939 & 18.65 \\
\hline & & $05-24-2001$ & 26.19 & & 12-11-1939 & 18.27 \\
\hline & & & & & 01-13-1940 & 17.91 \\
\hline (C-35-11)15dab-1 & 5,507 & $10-23-1973$ & 21 & & 02-13-1940 & 17.73 \\
\hline & & 03-20-1974 & 16 & & 03-13-1940 & 17.60 \\
\hline & & 08-18-1998 & 52.66 & & 04-03-1940 & 17.46 \\
\hline & & 11-18-1998 & 24.30 & & 05-03-1940 & 21.06 \\
\hline & & 03-17-1999 & 21.02 & & 04-02-1952 & 18.73 \\
\hline & & 11-01-1999 & 28.55 & & $12-07-1953$ & 22.39 \\
\hline & & $03-14-2000$ & 22.74 & & 03-09-1954 & 20.21 \\
\hline & & & & & $12-02-1954$ & 25.94 \\
\hline (C-35-11)16aab-1 & 5,494 & 03-20-1974 & 11.13 & & 03-20-1955 & 21.73 \\
\hline & & 03-17-1999 & 11.98 & & $10-11-1955$ & 32.68 \\
\hline & & 11-01-1999 & 21.80 & & $12-01-1955$ & 28.00 \\
\hline & & $03-14-2000$ & 14.62 & & 03-15-1956 & 24.34 \\
\hline & & & & & $10-18-1956$ & 40.56 \\
\hline (C-35-11)16ccc-1 & 5,510 & 03-20-1974 & 19.91 & & $12-12-1956$ & 30.52 \\
\hline & & 08-24-1998 & 70.50 & & 03-11-1957 & 27.38 \\
\hline & & 11-18-1998 & 35.59 & & $10-24-1957$ & 37.17 \\
\hline & & 03-17-1999 & 29.00 & & $12-03-1957$ & 30.11 \\
\hline & & 11-01-1999 & 44.36 & & 03-17-1958 & 26.48 \\
\hline & & 03-14-2000 & 33.46 & & $10-25-1958$ & 37.11 \\
\hline & & & & & $12-16-1958$ & 27.59 \\
\hline (C-35-11)17dcd-1 & 5,507 & 08-14-1937 & 19.99 & & 04-09-1959 & 24.57 \\
\hline & & 09-27-1937 & 18.96 & & $10-22-1959$ & 43.13 \\
\hline & & 12-03-1937 & 18.00 & & $12-07-1959$ & 33.47 \\
\hline & & 02-18-1938 & 17.55 & & 03-16-1960 & 28.98 \\
\hline & & 04-16-1938 & 16.92 & & $10-28-1960$ & 38.91 \\
\hline & & 05-17-1938 & 15.95 & & $12-05-1960$ & 35.08 \\
\hline & & 06-09-1938 & 15.91 & & 04-04-1961 & 30.60 \\
\hline & & 06-27-1938 & 16.65 & & 03-20-1962 & 32.20 \\
\hline & & 07-16-1938 & 17.18 & & $03-25-1963$ & 31.25 \\
\hline & & $07-27-1938$ & 17.62 & & 03-19-1964 & 34.00 \\
\hline & & 07-30-1938 & 17.66 & & 11-02-1964 & 49.09 \\
\hline & & 08-13-1938 & 17.70 & & 03-19-1965 & 35.34 \\
\hline & & 08-30-1938 & 18.18 & & $11-05-1965$ & 41.97 \\
\hline & & 09-09-1938 & 18.24 & & 03-10-1966 & 30.94 \\
\hline & & 09-28-1938 & 18.27 & & 03-06-1967 & 33.10 \\
\hline & & $11-02-1938$ & 17.67 & & 03-14-1969 & 28.02 \\
\hline & & $12-01-1938$ & 17.12 & & 03-11-1970 & 24.20 \\
\hline & & 01-03-1939 & 17.20 & & 03-08-1971 & 28.17 \\
\hline & & 02-02-1939 & 16.90 & & 03-08-1972 & 30.82 \\
\hline & & 03-02-1939 & 16.84 & & 03-15-1973 & 26.17 \\
\hline & & 04-04-1939 & 16.69 & & 03-11-1974 & 20.52 \\
\hline & & $04-22-1939$ & 16.44 & & 03-06-1975 & 29.09 \\
\hline
\end{tabular}


Table 2. Water levels in selected wells in Cedar Valley, Iron County, southwestern Utah—Continued

\begin{tabular}{|c|c|c|c|c|c|c|c|c|c|}
\hline Local well number & $\begin{array}{c}\text { Altitude of } \\
\text { land } \\
\text { surface } \\
\text { (feet) }\end{array}$ & Date & $\begin{array}{c}\text { Water leve } \\
\text { (feet) }\end{array}$ & & Local well number & $\begin{array}{c}\text { Altitude of } \\
\text { land } \\
\text { surface } \\
\text { (feet) }\end{array}$ & Date & $\begin{array}{c}\text { Water level } \\
\text { (feet) }\end{array}$ & \\
\hline \multirow[t]{21}{*}{ (C-35-11)17dcd-1-C } & tinued & 03-02-1976 & 25.49 & & (C-35-11)21 abd-1-C & tinued & 11-01-1999 & 39.80 & \\
\hline & & 10-19-1976 & 44.95 & & & & 03-16-2000 & 29.40 & \\
\hline & & 03-24-1977 & 30.22 & & & & & & \\
\hline & & 10-04-1977 & 59.25 & & (C-35-11)21cdb-2 & 5,533 & 06-01-1967 & 50 & $\mathrm{D}$ \\
\hline & & 03-02-1978 & 35.80 & & & & $11-19-1974$ & 43.90 & \\
\hline & & 03-15-1978 & 35.13 & & & & 08-14-1998 & 44.90 & \\
\hline & & 03-05-1979 & 31.49 & & & & 11-20-1998 & 39.68 & \\
\hline & & 03-07-1980 & 25.65 & & & & 03-17-1999 & 36.60 & \\
\hline & & 03-03-1981 & 20.36 & & & & 11-01-1999 & 42.46 & \\
\hline & & 03-01-1982 & 20.81 & & & & 03-16-2000 & 39.10 & \\
\hline & & 03-01-1983 & 18.46 & & & & & & \\
\hline & & 03-07-1984 & 9.82 & & (C-35-11)21dbd-2 & 5,534 & 09-13-1952 & 38.37 & \\
\hline & & 03-05-1985 & 8.53 & & & & $10-09-1952$ & 37.44 & \\
\hline & & 03-03-1986 & 8.62 & & & & $12-07-1953$ & 35.88 & \\
\hline & & 03-05-1987 & 9.71 & & & & 03-04-1954 & 33.09 & \\
\hline & & 03-01-1988 & 9.90 & & & & $12-02-1954$ & 39.16 & \\
\hline & & 03-09-1989 & 9.46 & & & & 03-18-1955 & 35.20 & \\
\hline & & 03-07-1990 & 16.70 & & & & 10-11-1955 & 48.55 & \\
\hline & & 03-01-1991 & 22.41 & & & & 12-01-1955 & 43.71 & \\
\hline & & 03-09-1992 & 25.77 & & & & 03-14-1956 & 38.66 & \\
\hline & & & & & & & 06-06-1956 & 41.03 & \\
\hline \multirow[t]{10}{*}{ (C-35-11)17dcd-2 } & 5,510 & 03-09-1994 & 26.21 & & & & 07-04-1956 & 42.06 & \\
\hline & & 03-08-1995 & 30.65 & & & & 08-07-1956 & 42.52 & \\
\hline & & 03-07-1996 & 23.38 & & & & 09-04-1956 & 43.10 & \\
\hline & & 03-04-1997 & 31.44 & & & & 10-17-1956 & 56.11 & \\
\hline & & 11-24-1997 & 39.87 & & & & $12-13-1956$ & 46.20 & \\
\hline & & 03-16-1998 & 32.18 & & & & 03-12-1957 & 43.27 & \\
\hline & & 11-17-1998 & 40.87 & & & & 04-30-1957 & 40.89 & \\
\hline & & 11-01-1999 & 50.55 & & & & 05-31-1957 & 41.83 & \\
\hline & & 03-08-2001 & 36.70 & & & & 10-24-1957 & 51.11 & \\
\hline & & & & & & & $12-03-1957$ & 45.39 & \\
\hline \multirow[t]{6}{*}{ (C-35-11)19bad-1 } & 5,505 & 08-25-1998 & 45.51 & & & & 03-17-1958 & 40.91 & \\
\hline & & 11-17-1998 & 47.01 & & & & 12-16-1958 & 41.28 & \\
\hline & & 03-17-1999 & 41.56 & & & & 04-09-1959 & 38.26 & \\
\hline & & 11-01-1999 & 60.13 & & & & 10-13-1959 & 61.34 & \\
\hline & & $03-15-2000$ & 46.86 & & & & 12-08-1959 & 48.82 & \\
\hline & & & & & & & 03-16-1960 & 44.59 & \\
\hline \multirow[t]{6}{*}{ (C-35-11)19dcc-1 } & 5,510 & 11-29-1995 & 54 & $\mathrm{D}$ & & & $10-28-1960$ & 55.78 & \\
\hline & & 11-17-1998 & 59.85 & & & & $12-06-1960$ & 52.33 & \\
\hline & & 03-17-1999 & 51.23 & & & & 04-04-1961 & 47.61 & \\
\hline & & 11-01-1999 & 67.25 & & & & $10-31-1961$ & 57.30 & \\
\hline & & $03-16-2000$ & 55.43 & & & & 03-20-1962 & 48.72 & \\
\hline & & & & & & & 10-10-1962 & 60.87 & \\
\hline \multirow[t]{5}{*}{ (C-35-11)20dda-1 } & 5,530 & $11-18-1998$ & 56.33 & & & & 03-25-1963 & 46.86 & \\
\hline & & 03-17-1999 & 40.72 & & & & $10-29-1963$ & 59.61 & \\
\hline & & 11-01-1999 & 61.09 & & & & 03-19-1964 & 47.83 & \\
\hline & & $03-15-2000$ & 46.97 & & & & 11-02-1964 & 66.37 & \\
\hline & & & & & & & 03-17-1965 & 52.40 & \\
\hline \multirow[t]{3}{*}{ (C-35-11)21abd-1 } & 5,520 & 07-19-1975 & 45 & $\mathrm{D}$ & & & 11-05-1965 & 55.00 & \\
\hline & & $11-20-1998$ & 31.06 & & & & 03-07-1966 & 46.49 & \\
\hline & & 03-17-1999 & 26.37 & & & & 03-06-1967 & 48.65 & \\
\hline
\end{tabular}


Table 2. Water levels in selected wells in Cedar Valley, Iron County, southwestern Utah—Continued

\begin{tabular}{|c|c|c|c|c|c|c|c|c|}
\hline Local well number & $\begin{array}{l}\text { Altitude of } \\
\text { land } \\
\text { surface } \\
\text { (feet) }\end{array}$ & Date & $\begin{array}{l}\text { Water level } \\
\text { (feet) }\end{array}$ & & Local well number & $\begin{array}{l}\text { Altitude of } \\
\text { land } \\
\text { surface } \\
\text { (feet) }\end{array}$ & Date & $\begin{array}{c}\text { Water level } \\
\text { (feet) }\end{array}$ \\
\hline \multirow{39}{*}{\multicolumn{2}{|c|}{ (C-35-11)21dbd-2-Continued }} & $03-12-1968$ & 44.27 & & (C-35-11)24bcc-1 & 5,585 & 08-17-1998 & 123.44 \\
\hline & & 03-14-1969 & 41.09 & & & & $11-18-1998$ & 117.99 \\
\hline & & 03-11-1970 & 35.68 & & & & 03-17-1999 & 113.16 \\
\hline & & 03-08-1971 & 41.46 & & & & 11-01-1999 & 118.61 \\
\hline & & 03-08-1972 & 42.97 & & & & 03-15-2000 & 115.57 \\
\hline & & 03-15-1973 & 43.50 & & & & & \\
\hline & & 03-11-1974 & 34.67 & & (C-35-11)25bcc-1 & 5,735 & 12-01-1999 & 258.69 \\
\hline & & 03-06-1975 & 41.68 & & & & 03-14-2000 & 262.01 \\
\hline & & 03-02-1976 & 39.69 & & & & $06-21-2000$ & 261.13 \\
\hline & & 03-01-1977 & 43.71 & & & & $06-22-2000$ & 261.34 \\
\hline & & 03-01-1978 & 53.91 & & & & $06-23-2000$ & 261.49 \\
\hline & & 03-15-1978 & 53.24 & & & & 09-21-2000 & 267.83 \\
\hline & & 03-05-1979 & 47.77 & & & & $12-13-2000$ & 267.05 \\
\hline & & 03-07-1980 & 39.99 & & & & 02-01-2001 & 266.21 \\
\hline & & 03-02-1981 & 33.05 & & & & 03-08-2001 & 263.43 \\
\hline & & 03-01-1982 & 33.67 & & & & 03-15-2001 & 265.37 \\
\hline & & 03-01-1983 & 30.53 & & & & & \\
\hline & & 03-30-1984 & 19.18 & & (C-35-11)26acd-1 & 5,670 & $11-29-1973$ & 183.76 \\
\hline & & 03-05-1985 & 17.08 & & & & 03-20-1974 & 177.51 \\
\hline & & 03-03-1986 & 18.14 & & & & 07-16-1998 & 184.35 \\
\hline & & 03-05-1987 & 19.55 & & & & $11-18-1998$ & 176.85 \\
\hline & & 03-01-1988 & 21.52 & & & & 03-17-1999 & 175.03 \\
\hline & & 03-09-1989 & 20.03 & & & & 11-01-1999 & 196.25 \\
\hline & & 03-07-1990 & 29.87 & & & & 03-14-2000 & 192.35 \\
\hline & & 03-01-1991 & 37.93 & & & & & \\
\hline & & 03-09-1992 & 41.79 & & (C-35-11)26dac-1 & 5,740 & 06-02-1999 & 236.17 \\
\hline & & 03-02-1993 & 42.69 & & & & 12-01-1999 & 239.28 \\
\hline & & 03-09-1994 & 37.00 & & & & 03-14-2000 & 237.89 \\
\hline & & 03-08-1995 & 43.75 & & & & & \\
\hline & & 03-07-1996 & 33.02 & & (C-35-11)27acc-1 & 5,556 & 06-25-1931 & 28.50 \\
\hline & & 03-04-1997 & 43.97 & & & & $11-02-1931$ & 35.00 \\
\hline & & $11-24-1997$ & 49.50 & & & & $12-03-1931$ & 34.00 \\
\hline & & 03-16-1998 & 43.88 & & & & 01-07-1932 & 34.00 \\
\hline & & 11-20-1998 & 42.86 & & & & 02-05-1932 & 33.00 \\
\hline & & 03-17-1999 & 38.04 & & & & 03-25-1932 & 32.00 \\
\hline & & 11-01-1999 & 51.48 & & & & $10-28-1932$ & 32.00 \\
\hline & & 03-16-2000 & 42.40 & & & & $11-28-1932$ & 31.00 \\
\hline & & 03-08-2001 & 48.32 & & & & $12-27-1932$ & 27.00 \\
\hline & & & & & & & 01-28-1933 & 31.00 \\
\hline \multirow[t]{6}{*}{ (C-35-11)23bdd-1 } & 5,550 & 08-17-1998 & 85.22 & & & & 02-27-1933 & 30.00 \\
\hline & & 11-18-1998 & 70.68 & & & & 03-30-1933 & 31.00 \\
\hline & & 03-17-1999 & 67.49 & & & & 05-31-1933 & 32.00 \\
\hline & & 11-01-1999 & 73.93 & & & & $12-28-1933$ & 34.00 \\
\hline & & 03-16-2000 & 69.18 & & & & 01-28-1934 & 33.00 \\
\hline & & & & & & & $12-08-1934$ & 39.00 \\
\hline \multirow[t]{7}{*}{ (C-35-11)24aab-1 } & 5,590 & $10-25-1973$ & 116.76 & & & & 01-29-1935 & 39.00 \\
\hline & & 03-20-1974 & 99 & $\mathrm{R}$ & & & 02-28-1935 & 39.00 \\
\hline & & 07-16-1998 & 116.10 & & & & 03-14-1935 & 39.00 \\
\hline & & 11-18-1998 & 113.77 & & & & 04-22-1935 & 39.00 \\
\hline & & 03-17-1999 & 108.29 & & & & 05-31-1935 & 39.00 \\
\hline & & 11-01-1999 & 119.52 & & & & 06-29-1935 & 39.00 \\
\hline & & $03-15-2000$ & 111.28 & & & & 07-20-1935 & 39.00 \\
\hline
\end{tabular}


Table 2. Water levels in selected wells in Cedar Valley, Iron County, southwestern Utah-Continued

\begin{tabular}{|c|c|c|c|c|c|c|c|}
\hline Local well number & $\begin{array}{c}\text { Altitude of } \\
\text { land } \\
\text { surface } \\
\text { (feet) }\end{array}$ & Date & $\begin{array}{c}\text { Water level } \\
\text { (feet) }\end{array}$ & Local well number & $\begin{array}{c}\text { Altitude of } \\
\text { land } \\
\text { surface } \\
\text { (feet) }\end{array}$ & Date & $\begin{array}{l}\text { Water level } \\
\text { (feet) }\end{array}$ \\
\hline \multirow{51}{*}{\multicolumn{2}{|c|}{ (C-35-11)27acc-1-Continued }} & 10-31-1935 & 43.00 & \multirow{51}{*}{\multicolumn{2}{|c|}{ (C-35-11)27acc-1-Continued }} & 10-30-1938 & 39.50 \\
\hline & & $11-26-1935$ & 43.00 & & & $11-12-1938$ & 38.82 \\
\hline & & $12-20-1935$ & 42.00 & & & $12-01-1938$ & 37.94 \\
\hline & & 01-29-1936 & 39.00 & & & 01-03-1939 & 37.12 \\
\hline & & 05-01-1936 & 39.00 & & & 01-14-1939 & 37.00 \\
\hline & & 05-28-1936 & 40.00 & & & 02-01-1939 & 36.73 \\
\hline & & 06-10-1936 & 46.15 & & & 03-02-1939 & 36.27 \\
\hline & & 09-26-1936 & 46.55 & & & 04-05-1939 & 35.89 \\
\hline & & $11-22-1936$ & 44.57 & & & 04-22-1939 & 35.68 \\
\hline & & $12-08-1936$ & 44.22 & & & 05-11-1939 & 37.46 \\
\hline & & $12-22-1936$ & 44.04 & & & 05-15-1939 & 38.08 \\
\hline & & 01-05-1937 & 43.90 & & & 09-23-1939 & 45.55 \\
\hline & & 01-20-1937 & 43.60 & & & 09-30-1939 & 45.79 \\
\hline & & 02-06-1937 & 42.90 & & & 10-09-1939 & 43.98 \\
\hline & & 02-20-1937 & 42.87 & & & $10-15-1939$ & 43.54 \\
\hline & & 03-06-1937 & 42.60 & & & $10-21-1939$ & 43.42 \\
\hline & & 03-20-1937 & 42.51 & & & $10-28-1939$ & 43.00 \\
\hline & & 04-03-1937 & 42.02 & & & $11-04-1939$ & 42.52 \\
\hline & & 04-17-1937 & 41.90 & & & $11-11-1939$ & 42.20 \\
\hline & & 05-01-1937 & 41.43 & & & $11-18-1939$ & 41.96 \\
\hline & & 05-15-1937 & 40.81 & & & $11-25-1939$ & 41.74 \\
\hline & & 05-29-1937 & 38.43 & & & $12-02-1939$ & 41.64 \\
\hline & & 06-07-1937 & 37.40 & & & $12-09-1939$ & 41.45 \\
\hline & & $06-12-1937$ & 37.06 & & & $12-13-1939$ & 41.33 \\
\hline & & $06-26-1937$ & 38.68 & & & $12-16-1939$ & 41.23 \\
\hline & & 07-11-1937 & 39.68 & & & $12-23-1939$ & 41.07 \\
\hline & & $10-16-1937$ & 42.90 & & & $12-30-1939$ & 40.95 \\
\hline & & $10-30-1937$ & 42.74 & & & $01-15-1940$ & 40.75 \\
\hline & & $11-13-1937$ & 41.19 & & & 02-13-1940 & 40.20 \\
\hline & & $11-27-1937$ & 40.62 & & & 03-13-1940 & 39.80 \\
\hline & & $12-04-1937$ & 40.42 & & & 04-03-1940 & 39.44 \\
\hline & & $12-18-1937$ & 40.10 & & & 05-08-1940 & 45.10 \\
\hline & & 01-01-1938 & 40.02 & & & $10-26-1940$ & 47.51 \\
\hline & & 01-15-1938 & 39.98 & & & $12-02-1940$ & 45.95 \\
\hline & & $01-29-1938$ & 39.58 & & & $12-28-1940$ & 45.18 \\
\hline & & 02-13-1938 & 39.50 & & & $01-25-1941$ & 44.62 \\
\hline & & 02-26-1938 & 39.29 & & & 02-25-1941 & 44.08 \\
\hline & & $03-12-1938$ & 39.17 & & & 03-24-1941 & 43.64 \\
\hline & & 03-26-1938 & 39.01 & & & 04-26-1941 & 43.20 \\
\hline & & 04-09-1938 & 38.75 & & & 05-24-1941 & 42.06 \\
\hline & & 04-23-1938 & 38.57 & & & 08-24-1941 & 41.87 \\
\hline & & 05-07-1938 & 38.22 & & & 09-27-1941 & 41.05 \\
\hline & & 05-19-1938 & 37.62 & & & $10-26-1941$ & 39.65 \\
\hline & & $05-21-1938$ & 37.45 & & & $11-25-1941$ & 38.18 \\
\hline & & 06-04-1938 & 37.25 & & & $12-02-1941$ & 37.97 \\
\hline & & 06-13-1938 & 35.65 & & & $12-25-1941$ & 37.27 \\
\hline & & $06-18-1938$ & 35.78 & & & $01-27-1942$ & 36.62 \\
\hline & & $08-17-1938$ & 41.90 & & & 02-25-1942 & 36.25 \\
\hline & & $08-29-1938$ & 42.66 & & & 03-16-1942 & 36.00 \\
\hline & & 09-29-1938 & 42.85 & & & 03-26-1942 & 35.88 \\
\hline & & $10-15-1938$ & 40.94 & & & $04-25-1942$ & 35.05 \\
\hline
\end{tabular}


Table 2. Water levels in selected wells in Cedar Valley, Iron County, southwestern Utah—Continued

\begin{tabular}{|c|c|c|c|c|c|c|c|}
\hline Local well number & $\begin{array}{c}\text { Altitude of } \\
\text { land } \\
\text { surface } \\
\text { (feet) }\end{array}$ & Date & $\begin{array}{c}\text { Water level } \\
\text { (feet) }\end{array}$ & Local well number & $\begin{array}{l}\text { Altitude of } \\
\text { land } \\
\text { surface } \\
\text { (feet) }\end{array}$ & Date & $\begin{array}{l}\text { Water level } \\
\text { (feet) }\end{array}$ \\
\hline$(\mathrm{C}-35-11) 27$ acc-1-C & tinued & 05-26-1942 & 31.72 & $(\mathrm{C}-35-11) 27 \mathrm{acc}-1-\mathrm{C}$ & tinued & 05-21-1947 & 41.87 \\
\hline & & 06-25-1942 & 30.24 & & & 06-08-1947 & 43.48 \\
\hline & & $10-24-1942$ & 35.20 & & & 07-01-1947 & 46.35 \\
\hline & & 11-26-1942 & 34.29 & & & 08-17-1947 & 49.24 \\
\hline & & $12-16-1942$ & 34.01 & & & 09-07-1947 & 48.41 \\
\hline & & $12-24-1942$ & 33.86 & & & 09-29-1947 & 47.98 \\
\hline & & 01-25-1943 & 33.47 & & & $11-02-1947$ & 45.10 \\
\hline & & $02-27-1943$ & 33.27 & & & $11-30-1947$ & 42.42 \\
\hline & & 03-22-1943 & 33.01 & & & $12-07-1947$ & 41.95 \\
\hline & & 04-24-1943 & 32.96 & & & $12-28-1947$ & 41.06 \\
\hline & & 05-26-1943 & 36.88 & & & 02-01-1948 & 40.06 \\
\hline & & $10-30-1943$ & 43.13 & & & 03-08-1948 & 39.33 \\
\hline & & $11-27-1943$ & 41.07 & & & 03-11-1948 & 40.31 \\
\hline & & $12-11-1943$ & 40.48 & & & 04-12-1948 & 38.54 \\
\hline & & $12-29-1943$ & 39.87 & & & 05-29-1948 & 40.06 \\
\hline & & 01-29-1944 & 39.00 & & & $07-15-1948$ & 53.72 \\
\hline & & 02-28-1944 & 38.22 & & & $07-30-1948$ & 54.67 \\
\hline & & 03-25-1944 & 37.48 & & & 08-28-1948 & 55.50 \\
\hline & & 04-29-1944 & 34.65 & & & 09-24-1948 & 56.40 \\
\hline & & 05-26-1944 & 35.96 & & & $10-30-1948$ & 47.19 \\
\hline & & 06-28-1944 & 35.67 & & & $11-28-1948$ & 44.20 \\
\hline & & 07-19-1944 & 44.82 & & & 04-18-1949 & 38.82 \\
\hline & & 07-30-1944 & 42.15 & & & 04-30-1949 & 38.32 \\
\hline & & 08-28-1944 & 46.51 & & & 05-31-1949 & 38.25 \\
\hline & & 09-29-1944 & 46.03 & & & 06-30-1949 & 35.49 \\
\hline & & $10-28-1944$ & 41.31 & & & $10-31-1949$ & 38.33 \\
\hline & & $12-02-1944$ & 41.03 & & & $11-29-1949$ & 37.22 \\
\hline & & $12-07-1944$ & 38.97 & & & 12-07-1949 & 36.96 \\
\hline & & $12-30-1944$ & 38.29 & & & 01-01-1950 & 36.46 \\
\hline & & 01-27-1945 & 37.48 & & & $01-31-1950$ & 36.14 \\
\hline & & 02-24-1945 & 36.72 & & & 02-01-1950 & 35.62 \\
\hline & & 03-27-1945 & 36.07 & & & 04-01-1950 & 35.15 \\
\hline & & 03-30-1945 & 35.97 & & & $05-02-1950$ & 35.06 \\
\hline & & 03-31-1945 & 35.90 & & & 06-01-1950 & 47.29 \\
\hline & & 04-28-1945 & 35.55 & & & 09-01-1950 & 55.78 \\
\hline & & 07-05-1945 & 45.09 & & & $10-02-1950$ & 56.34 \\
\hline & & 07-28-1945 & 47.13 & & & $10-27-1950$ & 47.18 \\
\hline & & 09-01-1945 & 42.60 & & & $12-03-1950$ & 44.88 \\
\hline & & $11-25-1945$ & 40.06 & & & $12-30-1950$ & 43.81 \\
\hline & & 12-09-1945 & 39.95 & & & 01-31-1951 & 42.76 \\
\hline & & $12-24-1945$ & 39.24 & & & 03-02-1951 & 42.70 \\
\hline & & 01-27-1946 & 38.73 & & & $03-31-1951$ & 41.96 \\
\hline & & 03-03-1946 & 37.31 & & & $10-31-1951$ & 52.36 \\
\hline & & 03-18-1946 & 36.96 & & & $11-30-1951$ & 52.42 \\
\hline & & 06-23-1946 & 49.80 & & & $12-06-1951$ & 50.29 \\
\hline & & $12-14-1946$ & 43.75 & & & 01-03-1952 & 49.40 \\
\hline & & 01-06-1947 & 42.66 & & & 02-05-1952 & 48.25 \\
\hline & & 01-26-1947 & 42.27 & & & $03-05-1952$ & 47.00 \\
\hline & & 02-24-1947 & 41.27 & & & 04-03-1952 & 46.47 \\
\hline & & 03-24-1947 & 40.20 & & & 04-04-1952 & 46.44 \\
\hline & & 03-30-1947 & 40.32 & & & 05-05-1952 & 45.19 \\
\hline
\end{tabular}


Table 2. Water levels in selected wells in Cedar Valley, Iron County, southwestern Utah—Continued

\begin{tabular}{|c|c|c|c|c|c|c|c|}
\hline Local well number & $\begin{array}{c}\text { Altitude of } \\
\text { land } \\
\text { surface } \\
\text { (feet) }\end{array}$ & Date & $\begin{array}{c}\text { Water level } \\
\text { (feet) }\end{array}$ & Local well number & $\begin{array}{l}\text { Altitude of } \\
\text { land } \\
\text { surface } \\
\text { (feet) }\end{array}$ & Date & $\begin{array}{l}\text { Water level } \\
\text { (feet) }\end{array}$ \\
\hline \multirow{51}{*}{\multicolumn{2}{|c|}{ (C-35-11)27acc-1-Continued }} & 06-04-1952 & 45.11 & \multirow{28}{*}{\multicolumn{2}{|c|}{ (C-35-11)27acc-1-Continued }} & $10-08-1970$ & 68.54 \\
\hline & & 07-02-1952 & 45.70 & & & 03-08-1971 & 57.59 \\
\hline & & $10-07-1952$ & 48.38 & & & 09-30-1971 & 67.83 \\
\hline & & $12-04-1952$ & 45.90 & & & 03-08-1972 & 61.17 \\
\hline & & $12-05-1952$ & 45.83 & & & 10-04-1972 & 73.05 \\
\hline & & 01-03-1953 & 45.05 & & & 10-10-1974 & 65.40 \\
\hline & & 02-07-1953 & 44.16 & & & 03-07-1975 & 63.67 \\
\hline & & 03-05-1953 & 43.66 & & & 10-08-1975 & 63.42 \\
\hline & & 03-13-1953 & 43.46 & & & 03-02-1976 & 56.98 \\
\hline & & 04-10-1953 & 43.13 & & & 10-08-1976 & 74.54 \\
\hline & & 05-07-1953 & 48.20 & & & 03-01-1977 & 62.15 \\
\hline & & $11-02-1953$ & 54.54 & & & 10-14-1977 & 81.30 \\
\hline & & $12-03-1953$ & 52.38 & & & 03-01-1978 & 68.40 \\
\hline & & $12-07-1953$ & 52.07 & & & 03-15-1978 & 67.73 \\
\hline & & 03-09-1954 & 49.24 & & & 03-08-1979 & 61.06 \\
\hline & & $12-02-1954$ & 55.30 & & & 03-07-1980 & 50.41 \\
\hline & & 03-18-1955 & 51.36 & & & $10-01-1980$ & 47.67 \\
\hline & & 12-01-1955 & 58.35 & & & 03-02-1981 & 44.35 \\
\hline & & 03-15-1956 & 55.15 & & & 03-01-1982 & 43.90 \\
\hline & & $10-30-1956$ & 66.61 & & & 10-01-1982 & 46.02 \\
\hline & & $12-13-1956$ & 62.35 & & & 03-01-1983 & 40.16 \\
\hline & & $03-12-1957$ & 58.92 & & & 09-29-1983 & 31.88 \\
\hline & & $10-24-1957$ & 64.55 & & & 03-06-1984 & 27.76 \\
\hline & & $12-03-1957$ & 61.25 & & & 09-26-1984 & 29.70 \\
\hline & & $03-17-1958$ & 57.34 & & & 03-05-1985 & 26.25 \\
\hline & & $10-27-1958$ & 60.40 & & & $10-21-1985$ & 30.69 \\
\hline & & $12-16-1958$ & 57.50 & & & 03-03-1986 & 28.28 \\
\hline & & 04-09-1959 & 54.38 & & & & \\
\hline & & 06-16-1959 & 57.70 & \multirow[t]{23}{*}{ (C-35-11)27bbc-1 } & \multirow[t]{23}{*}{5,547} & 05-18-1938 & 34.15 \\
\hline & & $10-20-1959$ & 69.75 & & & 06-13-1938 & 31.06 \\
\hline & & $12-08-1959$ & 65.00 & & & 07-15-1938 & 36.99 \\
\hline & & 03-16-1960 & 61.52 & & & 08-16-1938 & 35.35 \\
\hline & & $10-28-1960$ & 72.31 & & & 08-17-1938 & 35.39 \\
\hline & & $12-06-1960$ & 68.90 & & & 08-18-1938 & 35.45 \\
\hline & & 04-04-1961 & 64.41 & & & 08-19-1938 & 35.52 \\
\hline & & $10-16-1961$ & 73.12 & & & 09-12-1938 & 35.93 \\
\hline & & 03-20-1962 & 66.34 & & & 09-15-1939 & 40.97 \\
\hline & & $10-10-1962$ & 73.16 & & & $12-07-1953$ & 46.73 \\
\hline & & $03-25-1963$ & 64.45 & & & 03-03-1954 & 43.42 \\
\hline & & $10-29-1963$ & 75.35 & & & 03-18-1955 & 45.45 \\
\hline & & 03-19-1964 & 68.44 & & & $10-11-1955$ & 55.95 \\
\hline & & $11-02-1964$ & 77.36 & & & $12-01-1955$ & 53.44 \\
\hline & & 03-19-1965 & 69.24 & & & 03-15-1956 & 49.19 \\
\hline & & 03-07-1966 & 62.66 & & & $10-17-1956$ & 62.03 \\
\hline & & 09-27-1966 & 79.22 & & & $12-13-1956$ & 56.78 \\
\hline & & 03-06-1967 & 65.32 & & & 03-12-1957 & 53.15 \\
\hline & & 03-12-1968 & 61.47 & & & $10-24-1957$ & 59.36 \\
\hline & & $10-08-1968$ & 63.58 & & & $12-03-1957$ & 56.84 \\
\hline & & 03-14-1969 & 60.25 & & & $03-17-1958$ & 51.74 \\
\hline & & $10-08-1969$ & 58.55 & & & $10-27-1958$ & 55.17 \\
\hline & & $03-12-1970$ & 50.59 & & & $12-16-1958$ & 51.80 \\
\hline
\end{tabular}


Table 2. Water levels in selected wells in Cedar Valley, Iron County, southwestern Utah-Continued

\begin{tabular}{|c|c|c|c|c|c|c|c|c|}
\hline Local well number & $\begin{array}{l}\text { Altitude of } \\
\text { land } \\
\text { surface } \\
\text { (feet) }\end{array}$ & Date & $\begin{array}{c}\text { Water level } \\
\text { (feet) }\end{array}$ & Local well number & $\begin{array}{l}\text { Altitude of } \\
\text { land } \\
\text { surface } \\
\text { (feet) }\end{array}$ & Date & \multicolumn{2}{|l|}{$\begin{array}{l}\text { Water level } \\
\text { (feet) }\end{array}$} \\
\hline \multirow{49}{*}{\multicolumn{2}{|c|}{ (C-35-11)27bbc-1-Continued }} & 04-09-1959 & 48.42 & \multirow{3}{*}{\multicolumn{2}{|c|}{ (C-35-11)27bca-1-Continued }} & 11-01-1999 & 56.10 & \\
\hline & & $10-20-1959$ & 63.64 & & & 03-14-2000 & 52.10 & \\
\hline & & $12-08-1959$ & 59.19 & & & & & \\
\hline & & 03-16-1960 & 55.32 & \multirow[t]{6}{*}{ (C-35-11)27caa-1 } & \multirow[t]{6}{*}{5,560} & 08-14-1998 & 67.41 & \\
\hline & & $10-28-1960$ & 68.44 & & & $11-20-1998$ & 62.03 & \\
\hline & & $12-06-1960$ & 64.59 & & & 03-17-1999 & 60.47 & \\
\hline & & 04-04-1961 & 58.60 & & & 11-02-1999 & 70.39 & \\
\hline & & $10-16-1961$ & 68.80 & & & 03-14-2000 & 65.82 & \\
\hline & & 03-20-1962 & 61.62 & & & & & \\
\hline & & $10-25-1962$ & 67.77 & \multirow[t]{7}{*}{ (C-35-11)27dbb-1 } & \multirow[t]{7}{*}{5,560} & 08-19-1960 & 78 & $\mathrm{D}$ \\
\hline & & 03-25-1963 & 58.65 & & & 10-04-1974 & 70.03 & \\
\hline & & 03-19-1964 & 64.15 & & & $11-20-1998$ & 54.14 & \\
\hline & & $11-02-1964$ & 72.54 & & & 03-17-1999 & 53.66 & \\
\hline & & $11-05-1965$ & 64.07 & & & 11-02-1999 & 62.42 & \\
\hline & & 03-07-1966 & 60.37 & & & 03-14-2000 & 58.80 & \\
\hline & & 03-06-1967 & 62.72 & & & & & \\
\hline & & 03-12-1968 & 57.50 & \multirow[t]{18}{*}{ (C-35-11)28aac-2 } & \multirow[t]{18}{*}{5,548} & 03-05-1987 & 28.26 & \\
\hline & & 03-14-1969 & 52.93 & & & 03-01-1988 & 31.51 & \\
\hline & & 03-11-1970 & 46.85 & & & 03-09-1989 & 29.07 & \\
\hline & & 03-08-1971 & 52.46 & & & 03-01-1990 & 40.32 & \\
\hline & & 03-08-1972 & 52.55 & & & 03-01-1991 & 48.54 & \\
\hline & & 03-15-1973 & 54.60 & & & 03-09-1992 & 52.25 & \\
\hline & & 03-11-1974 & 43.87 & & & 03-02-1993 & 56.20 & \\
\hline & & 03-06-1975 & 53.66 & & & 03-09-1994 & 46.22 & \\
\hline & & 03-02-1976 & 52.18 & & & 03-08-1995 & 55.05 & \\
\hline & & $10-08-1976$ & 66.01 & & & 03-07-1996 & 43.02 & \\
\hline & & 03-01-1977 & 55.88 & & & 03-04-1997 & 54.55 & \\
\hline & & 03-01-1978 & 64.30 & & & $11-24-1997$ & 60.04 & \\
\hline & & 03-15-1978 & 63.42 & & & 03-16-1998 & 54.40 & \\
\hline & & 03-08-1979 & 56.92 & & & $11-20-1998$ & 52.18 & \\
\hline & & 03-07-1980 & 48.87 & & & 03-17-1999 & 48.00 & \\
\hline & & 03-02-1981 & 40.26 & & & 11-02-1999 & 60.28 & \\
\hline & & $10-01-1981$ & 48.35 & & & 03-14-2000 & 52.67 & \\
\hline & & 03-01-1982 & 41.12 & & & & & \\
\hline & & 03-01-1983 & 37.60 & \multirow[t]{5}{*}{ (C-35-11)28dac-1 } & \multirow[t]{5}{*}{5,562} & 11-20-1998 & 69.00 & \\
\hline & & 03-06-1984 & 24.49 & & & 03-17-1999 & 60.28 & \\
\hline & & 03-05-1985 & 22.52 & & & 11-02-1999 & 72.72 & \\
\hline & & 03-03-1986 & 24.54 & & & $03-14-2000$ & 65.28 & \\
\hline & & 03-15-1994 & 43.71 & & & & & \\
\hline & & 03-08-1995 & 51.00 & \multirow[t]{8}{*}{ (C-35-11)29acd-1 } & \multirow[t]{8}{*}{5,543} & $11-15-1954$ & 57 & $\mathrm{D}$ \\
\hline & & 03-07-1996 & 39.56 & & & $11-07-1973$ & 70.35 & \\
\hline & & 03-04-1997 & 52.63 & & & 08-24-1998 & 56.10 & \\
\hline & & $11-24-1997$ & 56.57 & & & $11-18-1998$ & 47.96 & \\
\hline & & 03-16-1998 & 52.14 & & & 03-17-1999 & 43.36 & \\
\hline & & 11-20-1998 & 48.96 & & & 11-02-1999 & 53.78 & \\
\hline & & 11-02-1999 & 55.08 & & & $03-14-2000$ & 47.38 & \\
\hline & & 03-14-2000 & 51.19 & & & & & \\
\hline & & 03-08-2001 & 59.50 & \multirow[t]{4}{*}{ (C-35-11)29bdc-1 } & \multirow[t]{4}{*}{5,535} & $11-18-1998$ & 51.34 & \\
\hline & & & & & & 03-17-1999 & 42.98 & \\
\hline \multirow[t]{2}{*}{ (C-35-11)27bca-1 } & \multirow[t]{2}{*}{5,550} & $11-20-1998$ & 48.94 & & & 11-02-1999 & 61.22 & \\
\hline & & 03-17-1999 & 47.30 & & & $03-14-2000$ & 47.33 & \\
\hline
\end{tabular}


Table 2. Water levels in selected wells in Cedar Valley, Iron County, southwestern Utah—Continued

\begin{tabular}{|c|c|c|c|c|c|c|}
\hline Local well number & $\begin{array}{l}\text { Altitude of } \\
\text { land } \\
\text { surface } \\
\text { (feet) }\end{array}$ & Date & $\begin{array}{l}\text { Water level } \\
\text { (feet) }\end{array}$ & $\begin{array}{l}\text { Altitude of } \\
\text { land } \\
\text { surface } \\
\text { (feet) }\end{array}$ & Date & $\begin{array}{c}\text { Water level } \\
\text { (feet) }\end{array}$ \\
\hline \multirow[t]{4}{*}{ (C-35-11)29cca-1 } & 5,540 & 03-17-1999 & 43.68 & (C-35-11)31acd-1-Continued & $11-23-1936$ & 26.95 \\
\hline & & $11-02-1999$ & 39.60 & & $12-.8-1936$ & 26.50 \\
\hline & & 03-15-2000 & 48.42 & & $12-22-1936$ & 28.55 \\
\hline & & & & & 03-20-1937 & 26.30 \\
\hline \multirow[t]{6}{*}{ (C-35-11)29dbd-2 } & 5,550 & 08-18-1998 & 61.86 & & 04-01-1937 & 23.18 \\
\hline & & $11-18-1998$ & 54.29 & & 04-03-1937 & 24.14 \\
\hline & & 03-17-1999 & 49.80 & & 04-17-1937 & 24.20 \\
\hline & & 11-02-1999 & 59.40 & & 05-01-1937 & 24.09 \\
\hline & & 03-14-2000 & 53.60 & & 05-29-1937 & 23.91 \\
\hline & & & & & 06-26-1937 & 25.60 \\
\hline \multirow[t]{41}{*}{ (C-35-11)31acd- 1} & 5,533 & $05-27-1930$ & 17 & & 09-27-1937 & 30.85 \\
\hline & & $10-02-1931$ & 17 & & $10-30-1937$ & 29.05 \\
\hline & & $11-10-1931$ & 17 & & $11-27-1937$ & 27.30 \\
\hline & & $12-04-1931$ & 17 & & $12-18-1937$ & 25.52 \\
\hline & & 01-09-1932 & 17 & & 01-29-1938 & 24.61 \\
\hline & & 02-17-1932 & 17 & & 02-26-1938 & 23.84 \\
\hline & & 03-26-1932 & 16 & & 03-26-1938 & 23.27 \\
\hline & & 04-22-1932 & 17 & & $04-23-1938$ & 22.77 \\
\hline & & 05-19-1932 & 17 & & $05-21-1938$ & 22.61 \\
\hline & & $06-22-1932$ & 17 & & 06-18-1938 & 23.58 \\
\hline & & $07-28-1932$ & 17 & & $09-27-1938$ & 27.68 \\
\hline & & 08-29-1932 & 20 & & $10-30-1938$ & 25.70 \\
\hline & & $09-30-1932$ & 16 & & $11-12-1938$ & 25.28 \\
\hline & & $10-26-1932$ & 17 & & $12-01-1938$ & 23.12 \\
\hline & & $11-30-1932$ & 17 & & 01-03-1939 & 22.13 \\
\hline & & $12-27-1932$ & 17 & & 02-02-1939 & 21.26 \\
\hline & & 01-00-1933 & 17 & & 03-03-1939 & 20.27 \\
\hline & & $02-27-1933$ & 17 & & 09-13-1939 & 30.72 \\
\hline & & $03-30-1933$ & 15 & & $10-21-1939$ & 27.52 \\
\hline & & $05-31-1933$ & 17 & & $12-11-1939$ & 24.99 \\
\hline & & $09-28-1933$ & 20 & & $01-15-1940$ & 24.71 \\
\hline & & $12-28-1933$ & 20 & & 02-14-1940 & 23.56 \\
\hline & & 01-00-1934 & 17 & & 03-13-1940 & 23.16 \\
\hline & & 05-00-1934 & 21 & & 04-04-1940 & 24.28 \\
\hline & & $10-31-1934$ & 24 & & 05-08-1940 & 27.86 \\
\hline & & $11-00-1934$ & 24 & & $12-02-1940$ & 28.47 \\
\hline & & $12-20-1934$ & 24 & & 03-24-1941 & 25.97 \\
\hline & & 01-28-1935 & 24 & & $12-01-1941$ & 23.00 \\
\hline & & 02-00-1935 & 24 & & 03-14-1942 & 18.05 \\
\hline & & 03-18-1935 & 24 & & $12-16-1942$ & 19.57 \\
\hline & & 04-22-1935 & 24 & & 03-23-1943 & 17.90 \\
\hline & & 05-31-1935 & 27 & & $12-11-1943$ & 22.65 \\
\hline & & 06-24-1935 & 27 & & 03-30-1945 & 19.73 \\
\hline & & $10-31-1935$ & 29 & & $12-09-1945$ & 22.91 \\
\hline & & $12-03-1935$ & 33 & & 03-18-1946 & 20.36 \\
\hline & & 01-29-1936 & 24.24 & & $12-14-1946$ & 26.56 \\
\hline & & 04-18-1936 & 22.99 & & 03-24-1947 & 23.65 \\
\hline & & 05-29-1936 & 24 & & $12-07-1947$ & 25.54 \\
\hline & & 06-16-1936 & 28.08 & & 03-11-1948 & 22.31 \\
\hline & & $08-02-1936$ & 29.55 & & $12-06-1948$ & 26.38 \\
\hline & & 09-25-1936 & 31.34 & & 04-22-1949 & 22.08 \\
\hline
\end{tabular}


Table 2. Water levels in selected wells in Cedar Valley, Iron County, southwestern Utah—Continued

\begin{tabular}{|c|c|c|c|c|c|c|c|}
\hline Local well number & $\begin{array}{c}\text { Altitude of } \\
\text { land } \\
\text { surface } \\
\text { (feet) }\end{array}$ & Date & $\begin{array}{c}\text { Water level } \\
\text { (feet) }\end{array}$ & Local well number & $\begin{array}{l}\text { Altitude of } \\
\text { land } \\
\text { surface } \\
\text { (feet) }\end{array}$ & Date & $\begin{array}{l}\text { Water level } \\
\text { (feet) }\end{array}$ \\
\hline \multirow{51}{*}{\multicolumn{2}{|c|}{ (C-35-11)31acd-1—Continued }} & $04-30-1949$ & 21.98 & \multicolumn{2}{|c|}{ (C-35-11)31 acd-1—Continued } & 04-07-1954 & 28.80 \\
\hline & & 05-31-1949 & 23.91 & & & 05-05-1954 & 36.30 \\
\hline & & 06-30-1949 & 25.04 & & & 06-03-1954 & 37.30 \\
\hline & & $10-31-1949$ & 27.06 & & & 07-07-1954 & 46.14 \\
\hline & & $11-29-1949$ & 22.81 & & & 08-06-1954 & 45.46 \\
\hline & & $12-07-1949$ & 22.32 & & & 09-03-1954 & 45.17 \\
\hline & & 01-01-1950 & 21.97 & & & 10-03-1954 & 44.16 \\
\hline & & $01-31-1950$ & 21.53 & & & 11-06-1954 & 40.55 \\
\hline & & 03-01-1950 & 21.04 & & & $12-03-1954$ & 39.15 \\
\hline & & 04-01-1950 & 19.74 & & & $12-04-1954$ & 36.78 \\
\hline & & $05-02-1950$ & 19.59 & & & 01-05-1955 & 32.88 \\
\hline & & $10-27-1950$ & 33.99 & & & 02-03-1955 & 30.61 \\
\hline & & $12-03-1950$ & 29.90 & & & 03-04-1955 & 31.90 \\
\hline & & $12-30-1950$ & 26.52 & & & 03-19-1955 & 31.94 \\
\hline & & 01-31-1951 & 26.44 & & & 04-05-1955 & 31.36 \\
\hline & & 03-02-1951 & 26.37 & & & 05-03-1955 & 35.77 \\
\hline & & 03-21-1951 & 24.49 & & & $10-11-1955$ & 46.19 \\
\hline & & 03-31-1951 & 26.36 & & & $12-02-1955$ & 38.69 \\
\hline & & 05-02-1951 & 26.55 & & & 05-20-1958 & 37.00 \\
\hline & & $12-06-1951$ & 32.94 & & & 07-02-1958 & 46.94 \\
\hline & & 01-03-1952 & 31.63 & & & $08-28-1958$ & 47.78 \\
\hline & & $02-05-1952$ & 30.03 & & & $10-09-1958$ & 44.90 \\
\hline & & 03-05-1952 & 29.00 & & & $10-24-1958$ & 43.52 \\
\hline & & 04-02-1952 & 28.23 & & & $12-16-1958$ & 38.22 \\
\hline & & 04-04-1952 & 28.26 & & & $10-22-1959$ & 51.86 \\
\hline & & 05-05-1952 & 27.30 & & & $12-10-1959$ & 44.12 \\
\hline & & 06-04-1952 & 26.67 & & & 03-15-1960 & 40.25 \\
\hline & & 07-02-1952 & 30.66 & & & $10-28-1960$ & 52.30 \\
\hline & & 08-05-1952 & 39.72 & & & $12-02-1960$ & 48.07 \\
\hline & & 09-03-1952 & 38.92 & & & 04-04-1961 & 43.09 \\
\hline & & $11-06-1952$ & 33.40 & & & $10-31-1961$ & 50.63 \\
\hline & & $12-04-1952$ & 27.60 & & & 03-20-1962 & 46.70 \\
\hline & & $12-05-1952$ & 27.48 & & & $10-10-1962$ & 62.12 \\
\hline & & 01-03-1953 & 26.64 & & & 03-25-1963 & 44.15 \\
\hline & & 02-07-1953 & 25.65 & & & 03-19-1964 & 48.48 \\
\hline & & 03-05-1953 & 25.34 & & & 03-19-1965 & 49.27 \\
\hline & & 03-13-1953 & 25.19 & & & $11-05-1965$ & 54.11 \\
\hline & & 04-10-1953 & 28.25 & & & 03-07-1966 & 44.66 \\
\hline & & 05-07-1953 & 30.66 & & & $12-01-1972$ & 45.00 \\
\hline & & 06-05-1953 & 40.92 & & & $03-15-1973$ & 39.02 \\
\hline & & 07-03-1953 & 41.31 & & & 03-11-1974 & 29.79 \\
\hline & & 08-01-1953 & 41.72 & & & 03-05-1975 & 33.81 \\
\hline & & 09-02-1953 & 44.82 & & & $10-08-1975$ & 54.46 \\
\hline & & $10-05-1953$ & 45.12 & & & 03-02-1976 & 33.21 \\
\hline & & $11-02-1953$ & 38.75 & & & $10-08-1976$ & 57.89 \\
\hline & & $12-03-1953$ & 33.11 & & & 03-01-1977 & 37.26 \\
\hline & & $12-07-1953$ & 32.62 & & & & \\
\hline & & 01-05-1954 & 31.06 & (C-35-11)31acd-2 & 5,536 & 08-03-1951 & 61.58 \\
\hline & & 02-04-1954 & 29.75 & & & $08-31-1951$ & 63.41 \\
\hline & & 03-02-1954 & 29.26 & & & 10-03-1951 & 62.60 \\
\hline & & 03-09-1954 & 28.94 & & & $10-31-1951$ & 37.98 \\
\hline
\end{tabular}


Table 2. Water levels in selected wells in Cedar Valley, Iron County, southwestern Utah—Continued

\begin{tabular}{|c|c|c|c|c|c|c|c|c|c|}
\hline Local well number & $\begin{array}{l}\text { Altitude of } \\
\text { land } \\
\text { surface } \\
\text { (feet) }\end{array}$ & Date & $\begin{array}{c}\text { Water level } \\
\text { (feet) }\end{array}$ & & Local well number & $\begin{array}{l}\text { Altitude of } \\
\text { land } \\
\text { surface } \\
\text { (feet) }\end{array}$ & Date & $\begin{array}{l}\text { Water level } \\
\text { (feet) }\end{array}$ & \\
\hline$(\mathrm{C}-35-11) 31$ acd $-2-\mathrm{C}$ & tinued & $11-30-1951$ & 37.46 & & $\mathrm{C}-35-11) 31$ acd-2-C & nued & $11-25-1997$ & 58.62 & \\
\hline & & 04-30-1955 & 43 & $\mathrm{D}$ & & & 03-16-1998 & 48.30 & \\
\hline & & $12-12-1956$ & 43.99 & & & & $11-17-1998$ & 51.40 & \\
\hline & & 03-11-1957 & 41.07 & & & & 03-17-1999 & 42.69 & \\
\hline & & $10-23-1957$ & 52.38 & & & & 11-02-1999 & 63.28 & \\
\hline & & 12-03-1957 & 45.95 & & & & 03-14-2000 & 48.41 & \\
\hline & & 03-17-1958 & 40.08 & & & & 03-09-2001 & 54.19 & \\
\hline & & $05-20-1958$ & 38.64 & & & & & & \\
\hline & & $10-24-1958$ & 50.11 & & $(\mathrm{C}-35-11) 31$ bac- 1 & 5,520 & 03-16-1995 & 52 & $\mathrm{D}$ \\
\hline & & 09-07-1966 & 70.51 & & & & $11-19-1998$ & 55.63 & \\
\hline & & 03-06-1967 & 49.08 & & & & 03-17-1999 & 37.30 & \\
\hline & & $10-06-1967$ & 59.98 & & & & 11-02-1999 & 63.32 & \\
\hline & & 03-26-1968 & 44.83 & & & & $03-14-2000$ & 48.90 & \\
\hline & & 03-14-1969 & 42.00 & & & & & & \\
\hline & & 03-11-1970 & 36.47 & & (C-35-11)31dbd-1 & 5,535 & 04-11-1977 & 51 & $\mathrm{D}$ \\
\hline & & 03-08-1971 & 42.35 & & & & 11-17-1998 & 56.26 & \\
\hline & & 03-09-1972 & 42.59 & & & & 03-17-1999 & 48.03 & \\
\hline & & $10-04-1972$ & 68.06 & & & & 11-02-1999 & 67.50 & \\
\hline & & $12-01-1972$ & 52.65 & & & & 03-14-2000 & 52.62 & \\
\hline & & 03-15-1973 & 42.85 & & & & & & \\
\hline & & 03-11-1974 & 32.51 & & (C-35-11)32aad-1 & 5,560 & 09-07-1955 & 76 & $\mathrm{D}$ \\
\hline & & $10-10-1974$ & 58.59 & & & & 03-17-1999 & 57.92 & \\
\hline & & 03-05-1975 & 39.67 & & & & 11-02-1999 & 72.16 & \\
\hline & & 03-02-1976 & 39.54 & & & & 03-14-2000 & 62.68 & \\
\hline & & $10-08-1976$ & 70.23 & & & & & & \\
\hline & & 03-01-1977 & 45.70 & & (C-35-11)32abd-1 & 5,553 & 11-08-1974 & 70 & $\mathrm{R}$ \\
\hline & & 10-04-1977 & 78.63 & & & & 05-21-1998 & 68.17 & \\
\hline & & 03-01-1978 & 54.01 & & & & 11-18-1998 & 62.96 & \\
\hline & & $03-15-1978$ & 53.32 & & & & 03-17-1999 & 55.75 & \\
\hline & & $10-03-1978$ & 67.80 & & & & 11-02-1999 & 71.75 & \\
\hline & & 03-08-1979 & 48.57 & & & & $03-14-2000$ & 60.48 & \\
\hline & & 03-07-1980 & 40.95 & & & & & & \\
\hline & & 03-03-1981 & 33.72 & & (C-35-11)32bda-1 & 5,547 & 04-19-1975 & 55 & $\mathrm{D}$ \\
\hline & & 03-01-1982 & 35.00 & & & & 05-20-1998 & 80.40 & \\
\hline & & 03-01-1983 & 31.90 & & & & $11-20-1998$ & 63.58 & \\
\hline & & 03-06-1984 & 21.90 & & & & 03-17-1999 & 55.87 & \\
\hline & & 09-26-1984 & 33.29 & & & & 11-02-1999 & 74.94 & \\
\hline & & 03-05-1985 & 20.49 & & & & $03-14-2000$ & 60.60 & \\
\hline & & 03-03-1986 & 21.42 & & & & & & \\
\hline & & 03-05-1987 & 22.48 & & (C-35-11)32dba-1 & 5,555 & 11-19-1998 & 68.58 & \\
\hline & & 03-01-1988 & 24.02 & & & & 03-17-1999 & 61.23 & \\
\hline & & 03-09-1989 & 22.53 & & & & & & \\
\hline & & 03-01-1990 & 31.97 & & $(\mathrm{C}-35-11) 33 \mathrm{aac}-1$ & 5,576 & 08-20-1931 & 58.40 & \\
\hline & & 09-22-1990 & 75.80 & & & & $10-08-1931$ & 57.70 & \\
\hline & & 03-01-1991 & 39.69 & & & & $11-05-1931$ & 56.50 & \\
\hline & & 03-09-1992 & 44.74 & & & & 01-08-1932 & 56.00 & \\
\hline & & 03-03-1993 & 46.35 & & & & 02-18-1932 & 56.00 & \\
\hline & & 03-09-1994 & 39.27 & & & & $03-31-1932$ & 53.50 & \\
\hline & & 03-08-1995 & 48.59 & & & & 04-23-1932 & 53.70 & \\
\hline & & 03-07-1996 & 38.45 & & & & $05-27-1932$ & 52.30 & \\
\hline & & 03-04-1997 & 46.35 & & & & 06-23-1932 & 52.00 & \\
\hline
\end{tabular}


Table 2. Water levels in selected wells in Cedar Valley, Iron County, southwestern Utah—Continued

\begin{tabular}{|c|c|c|c|c|c|c|}
\hline Local well number & $\begin{array}{c}\text { Altitude of } \\
\text { land } \\
\text { surface } \\
\text { (feet) }\end{array}$ & Date & $\begin{array}{c}\text { Water level } \\
\text { (feet) }\end{array}$ & $\begin{array}{c}\text { Altitude of } \\
\text { land } \\
\text { surface } \\
\text { (feet) }\end{array}$ & Date & $\begin{array}{c}\text { Water level } \\
\text { (feet) }\end{array}$ \\
\hline \multirow{51}{*}{\multicolumn{2}{|c|}{ (C-35-11)33aac-1-Continued }} & 09-30-1932 & 53.90 & \multirow[t]{51}{*}{ (C-35-11)33aac-1-Continued } & 04-09-1938 & 61.68 \\
\hline & & $10-28-1932$ & 53.70 & & 04-16-1938 & 61.42 \\
\hline & & $11-30-1932$ & 55.00 & & 04-23-1938 & 61.13 \\
\hline & & $12-27-1932$ & 55.00 & & 05-07-1938 & 61.09 \\
\hline & & 03-30-1933 & 52.20 & & 05-21-1938 & 59.53 \\
\hline & & 04-28-1933 & 53.50 & & 06-04-1938 & 59.62 \\
\hline & & 09-28-1933 & 60.00 & & 06-13-1938 & 59.20 \\
\hline & & $12-28-1933$ & 56.00 & & 09-29-1938 & 65.00 \\
\hline & & $10-25-1934$ & 67.00 & & $10-15-1938$ & 63.63 \\
\hline & & $12-20-1934$ & 64.50 & & $10-30-1938$ & 63.00 \\
\hline & & 01-29-1935 & 65.00 & & $11-12-1938$ & 62.02 \\
\hline & & 03-18-1935 & 62.20 & & $12-01-1938$ & 61.80 \\
\hline & & 04-22-1935 & 63.00 & & 01-04-1939 & 60.64 \\
\hline & & 05-31-1935 & 62.00 & & 02-01-1939 & 59.85 \\
\hline & & 06-29-1935 & 64.00 & & 03-06-1939 & 59.10 \\
\hline & & 07-27-1935 & 64.30 & & 04-05-1939 & 58.50 \\
\hline & & $10-31-1935$ & 67.50 & & 04-22-1939 & 58.06 \\
\hline & & $12-28-1935$ & 64.00 & & 09-08-1939 & 69.08 \\
\hline & & 01-29-1936 & 63.62 & & 09-13-1939 & 68.33 \\
\hline & & 02-02-1936 & 63.40 & & $10-20-1939$ & 66.42 \\
\hline & & 05-13-1936 & 62.64 & & $12-13-1939$ & 64.67 \\
\hline & & 05-29-1936 & 62.10 & & 01-15-1940 & 63.79 \\
\hline & & 09-26-1936 & 71.00 & & 02-14-1940 & 63.09 \\
\hline & & $11-23-1936$ & 67.90 & & 03-15-1940 & 62.09 \\
\hline & & $12-08-1936$ & 67.59 & & 09-13-1940 & 72.70 \\
\hline & & $12-22-1936$ & 67.18 & & $12-02-1940$ & 69.15 \\
\hline & & 01-04-1937 & 67.04 & & 03-24-1941 & 66.15 \\
\hline & & 01-21-1937 & 67.00 & & $12-02-1941$ & 62.00 \\
\hline & & 02-06-1937 & 66.60 & & 03-16-1942 & 58.40 \\
\hline & & 02-20-1937 & 66.91 & & $12-16-1942$ & 57.83 \\
\hline & & 03-06-1937 & 64.81 & & $03-22-1943$ & 55.70 \\
\hline & & 03-20-1937 & 64.57 & & $12-11-1943$ & 63.13 \\
\hline & & 04-03-1937 & 64.09 & & $12-07-1944$ & 62.43 \\
\hline & & 04-17-1937 & 63.90 & & 03-30-1945 & 58.15 \\
\hline & & 05-01-1937 & 63.65 & & $12-09-1945$ & 62.89 \\
\hline & & 05-15-1937 & 63.00 & & 03-18-1946 & 59.62 \\
\hline & & 05-29-1937 & 60.15 & & $12-14-1946$ & 66.79 \\
\hline & & $06-12-1937$ & 59.85 & & 03-24-1947 & 63.12 \\
\hline & & 08-21-1937 & 68.24 & & $12-07-1947$ & 65.70 \\
\hline & & $10-30-1937$ & 66.40 & & 03-11-1948 & 62.35 \\
\hline & & 11-13-1937 & 66.30 & & $12-06-1948$ & 67.57 \\
\hline & & $11-27-1937$ & 65.79 & & 04-18-1949 & 61.52 \\
\hline & & $12-03-1937$ & 64.76 & & 04-30-1949 & 61.00 \\
\hline & & $12-18-1937$ & 63.94 & & 05-31-1949 & 60.65 \\
\hline & & 01-01-1938 & 63.97 & & $12-07-1949$ & 61.71 \\
\hline & & 01-15-1938 & 63.52 & & 01-01-1950 & 60.70 \\
\hline & & 01-29-1938 & 63.30 & & $01-31-1950$ & 60.12 \\
\hline & & 02-13-1938 & 63.20 & & 03-01-1950 & 58.36 \\
\hline & & 02-26-1938 & 62.92 & & 03-22-1950 & 58.17 \\
\hline & & 03-12-1938 & 62.43 & & 04-01-1950 & 58.21 \\
\hline & & 03-26-1938 & 62.84 & & $10-02-1950$ & 71.14 \\
\hline
\end{tabular}


Table 2. Water levels in selected wells in Cedar Valley, Iron County, southwestern Utah-Continued

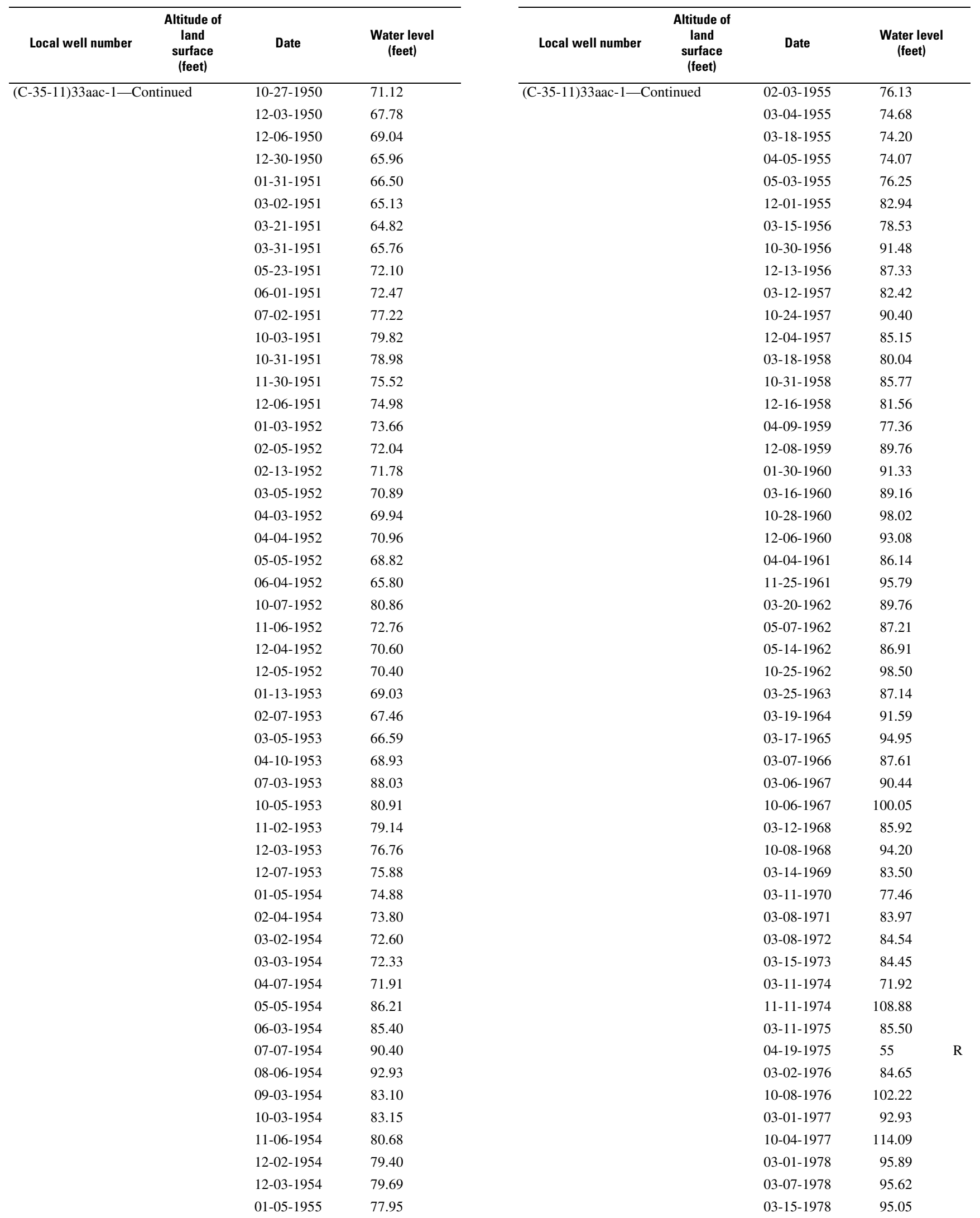


Table 2. Water levels in selected wells in Cedar Valley, Iron County, southwestern Utah-Continued

\begin{tabular}{|c|c|c|c|c|c|c|c|c|c|}
\hline Local well number & $\begin{array}{l}\text { Altitude of } \\
\text { land } \\
\text { surface } \\
\text { (feet) }\end{array}$ & Date & $\begin{array}{c}\text { Water leve } \\
\text { (feet) }\end{array}$ & & Local well number & $\begin{array}{l}\text { Altitude of } \\
\text { land } \\
\text { surface } \\
\text { (feet) }\end{array}$ & Date & $\begin{array}{c}\text { Water level } \\
\text { (feet) }\end{array}$ & \\
\hline \multirow{38}{*}{\multicolumn{2}{|c|}{ (C-35-11)33aac-1-Continued }} & 03-03-1979 & 88.17 & & $(\mathrm{C}-35-11) 33 \mathrm{dbd}-2$ & 5,585 & 07-16-1998 & 93.80 & \\
\hline & & 05-23-1979 & 84.10 & & & & $11-20-1998$ & 93.93 & \\
\hline & & 10-01-1979 & 95.55 & & & & 03-17-1999 & 86.35 & \\
\hline & & 03-07-1980 & 79.49 & & & & 11-02-1999 & 102.17 & \\
\hline & & $10-01-1980$ & 82.80 & & & & 03-15-2000 & 91.40 & \\
\hline & & 03-02-1981 & 72.44 & & & & & & \\
\hline & & 03-01-1982 & 73.62 & & (C-35-11)34abb-1 & 5,575 & 07-29-1961 & 103 & $\mathrm{D}$ \\
\hline & & 10-01-1982 & 80.20 & & & & 08-14-1998 & 73.20 & \\
\hline & & 03-01-1983 & 68.76 & & & & $11-20-1998$ & 74.05 & \\
\hline & & 09-29-1983 & 70.05 & & & & 03-17-1999 & 72.46 & \\
\hline & & 03-05-1984 & 58.03 & & & & 11-03-1999 & 82.28 & \\
\hline & & 09-26-1984 & 63.65 & & & & 03-15-2000 & 77.69 & \\
\hline & & 03-05-1985 & 57.70 & & & & & & \\
\hline & & $10-21-1985$ & 62.85 & & (C-35-11)34dbb-1 & 5,600 & $11-20-1998$ & 104.86 & \\
\hline & & 03-03-1986 & 58.07 & & & & 03-17-1999 & 100.67 & \\
\hline & & 09-29-1986 & 65.99 & & & & 11-03-1999 & 113.08 & \\
\hline & & 03-05-1987 & 59.75 & & & & 03-15-2000 & 106.02 & \\
\hline & & 09-17-1987 & 70.77 & & & & & & \\
\hline & & 03-01-1988 & 61.75 & & (C-35-12)18ddd-4 & 5,382 & 03-19-1969 & 9.22 & \\
\hline & & 09-19-1988 & 74.84 & & & & 03-02-1976 & 9.04 & \\
\hline & & 03-09-1989 & 59.63 & & & & 08-16-1976 & 30.71 & \\
\hline & & 09-22-1989 & 79.57 & & & & $10-12-1976$ & 23.39 & \\
\hline & & 03-01-1990 & 72.82 & & & & $12-21-1976$ & 13.56 & \\
\hline & & 03-01-1991 & 85.50 & & & & 02-12-1977 & 11.20 & \\
\hline & & 09-18-1991 & 104.22 & & & & 03-05-1977 & 10.85 & \\
\hline & & 03-09-1992 & 84.87 & & & & 06-02-1977 & 34.59 & \\
\hline & & 03-02-1993 & 88.34 & & & & 09-07-1977 & 21.40 & \\
\hline & & 03-04-1994 & 77.45 & & & & $11-11-1977$ & 26.17 & \\
\hline & & 03-08-1995 & 84.16 & & & & $12-15-1977$ & 13.70 & \\
\hline & & 03-07-1996 & 74.85 & & & & 02-01-1978 & 9.70 & \\
\hline & & 03-04-1997 & 85.65 & & & & 03-04-1978 & 8.55 & \\
\hline & & $11-24-1997$ & 93.85 & & & & $10-02-1978$ & 33.63 & \\
\hline & & 03-16-1998 & 86.15 & & & & 03-06-1979 & 8.99 & \\
\hline & & 03-23-1999 & 79.15 & & & & $10-02-1980$ & 9.96 & \\
\hline & & 11-05-1999 & 93.13 & & & & 03-03-1981 & 8.44 & \\
\hline & & 03-15-2000 & 84.30 & & & & $10-01-1981$ & 9.74 & \\
\hline & & 03-09-2001 & 90.58 & & & & 03-01-1982 & 10.07 & \\
\hline & & & & & & & 10-01-1982 & 10.70 & \\
\hline \multirow[t]{7}{*}{ (C-35-11)33bcc-1 } & 5,563 & 03-20-1940 & 51.8 & $\mathrm{D}$ & & & 03-01-1983 & 10.13 & \\
\hline & & 05-21-1998 & 76.94 & & & & $10-11-1983$ & 11.55 & \\
\hline & & 11-20-1998 & 73.55 & & & & 03-02-1984 & 11.18 & \\
\hline & & 03-17-1999 & 66.00 & & & & 03-05-1985 & 12.80 & \\
\hline & & 11-03-1999 & 81.83 & & & & 10-01-1985 & 11.80 & \\
\hline & & 03-15-2000 & 71.17 & & & & 03-03-1986 & 11.63 & \\
\hline & & & & & & & $10-01-1986$ & 12.15 & \\
\hline \multirow[t]{6}{*}{ (C-35-11)33ccd-1 } & 5,575 & $12-30-1971$ & 88 & D & & & 03-02-1987 & 12.08 & \\
\hline & & 03-21-1974 & 59 & $\mathrm{R}$ & & & 09-18-1987 & 13.49 & \\
\hline & & $11-18-1998$ & 88.60 & & & & 03-08-1988 & 12.85 & \\
\hline & & 03-17-1999 & 79.93 & & & & 03-07-1989 & 14.02 & \\
\hline & & 11-02-1999 & 96.90 & & & & 09-28-1989 & 15.20 & \\
\hline & & $03-15-2000$ & 84.82 & & & & 03-02-1990 & 14.88 & \\
\hline
\end{tabular}


Table 2. Water levels in selected wells in Cedar Valley, Iron County, southwestern Utah—Continued

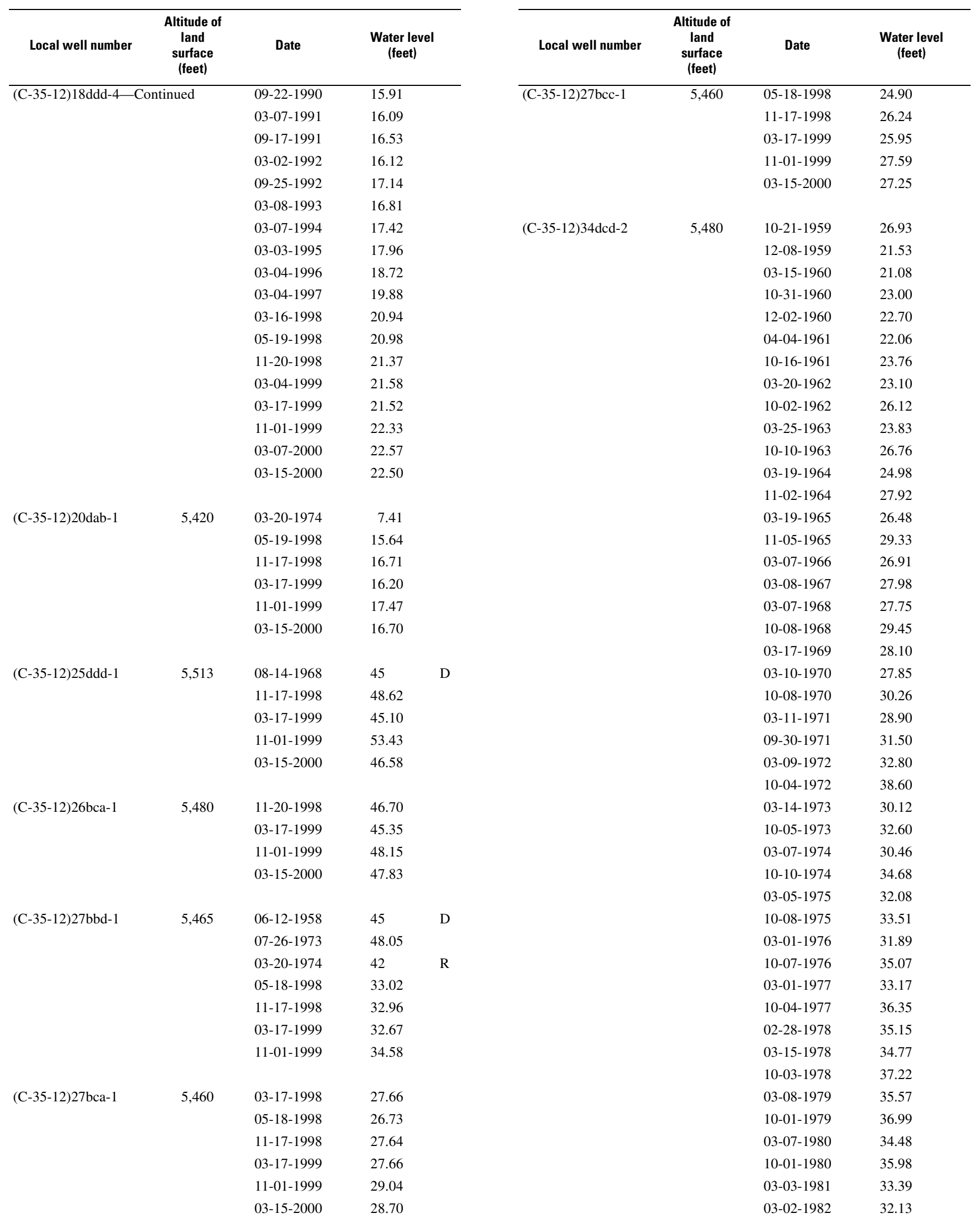


Table 2. Water levels in selected wells in Cedar Valley, Iron County, southwestern Utah-Continued

\begin{tabular}{|c|c|c|c|c|c|c|c|c|}
\hline Local well number & $\begin{array}{l}\text { Altitude of } \\
\text { land } \\
\text { surface } \\
\text { (feet) }\end{array}$ & Date & $\begin{array}{c}\text { Water level } \\
\text { (feet) }\end{array}$ & Local well number & $\begin{array}{l}\text { Altitude of } \\
\text { land } \\
\text { surface } \\
\text { (feet) }\end{array}$ & Date & \multicolumn{2}{|c|}{$\begin{array}{l}\text { Water level } \\
\text { (feet) }\end{array}$} \\
\hline \multirow{34}{*}{\multicolumn{2}{|c|}{ (C-35-12)34dcd-2-Continued }} & $10-01-1982$ & 32.95 & $(\mathrm{C}-35-12) 36$ caa-2-C & tinued & 06-08-2001 & 19.67 & \\
\hline & & 03-02-1983 & 31.25 & & & 06-14-2001 & 19.49 & \\
\hline & & $09-27-1983$ & 32.10 & & & & & \\
\hline & & 03-05-1984 & 29.30 & (C-35-12)36ddd-1 & 5,515 & 03-28-1994 & 22 & D \\
\hline & & 09-26-1984 & 30.80 & & & 03-10-1995 & 30.99 & \\
\hline & & 03-05-1985 & 28.03 & & & 03-07-1996 & 23.94 & \\
\hline & & $10-21-1985$ & 29.02 & & & 03-04-1997 & 32.94 & \\
\hline & & 03-03-1986 & 27.55 & & & $11-25-1997$ & 43.20 & \\
\hline & & $09-29-1986$ & 28.20 & & & 03-16-1998 & 34.60 & \\
\hline & & 03-12-1987 & 27.50 & & & $11-17-1998$ & 38.28 & \\
\hline & & 09-17-1987 & 30.07 & & & 03-17-1999 & 29.54 & \\
\hline & & 03-02-1988 & 26.89 & & & 11-01-1999 & 49.49 & \\
\hline & & 09-19-1988 & 29.82 & & & 03-15-2000 & 34.16 & \\
\hline & & 03-08-1989 & 25.29 & & & & & \\
\hline & & 09-22-1989 & 31.26 & (C-36-11)3bda-1 & 5,640 & 07-15-1998 & 137.09 & \\
\hline & & 03-02-1990 & 28.38 & & & 11-19-1998 & 145.74 & \\
\hline & & $09-22-1990$ & 38.08 & & & 03-17-1999 & 138.80 & \\
\hline & & 03-01-1991 & 35.89 & & & 11-02-1999 & 152.21 & \\
\hline & & 09-18-1991 & 42.48 & & & $03-14-2000$ & 144.59 & \\
\hline & & 03-09-1992 & 34.10 & & & 03-09-2001 & 156.32 & \\
\hline & & 09-28-1992 & 37.35 & & & & & \\
\hline & & 03-08-1993 & 33.30 & (C-36-11)5cba-1 & 5,550 & 11-19-1998 & 64.40 & \\
\hline & & 03-09-1994 & 33.76 & & & 03-16-1999 & 55.44 & \\
\hline & & 03-09-1995 & 39.03 & & & 11-02-1999 & 72.84 & \\
\hline & & 03-08-1996 & 40.76 & & & $03-15-2000$ & 60.22 & \\
\hline & & 03-04-1997 & 43.45 & & & & & \\
\hline & & $11-25-1997$ & 54.86 & $(\mathrm{C}-36-11) 5 \mathrm{dcc}-1$ & 5,555 & 05-21-1957 & 72 & $\mathrm{D}$ \\
\hline & & 03-16-1998 & 41.54 & & & 03-14-2000 & 70.68 & \\
\hline & & $11-17-1998$ & 55.83 & & & & & \\
\hline & & 03-17-1999 & 41.82 & (C-36-11)7aba-1 & 5,532 & 04-27-1965 & 54 & $\mathrm{D}$ \\
\hline & & 11-01-1999 & 50.07 & & & 05-20-1998 & 73.60 & \\
\hline & & 03-15-2000 & 44.50 & & & 11-19-1998 & 62.93 & \\
\hline & & 03-09-2001 & 51.47 & & & 03-16-1999 & 55.14 & \\
\hline & & & & & & 11-02-1999 & 73.15 & \\
\hline \multirow[t]{4}{*}{ (C-35-12)36аaа-1 } & 5,515 & 03-17-1999 & 56.66 & & & $03-15-2000$ & 58.79 & \\
\hline & & 11-01-1999 & 68.35 & & & & & \\
\hline & & 03-15-2000 & 58.60 & (C-36-11)7cab-1 & 5,522 & 09-05-1961 & 54 & $\mathrm{D}$ \\
\hline & & & & & & 05-18-1998 & 64.84 & \\
\hline \multirow[t]{6}{*}{ (C-35-12)36caa-1 } & 5,505 & $11-17-1998$ & 58.50 & & & 11-02-1999 & 68.69 & \\
\hline & & 03-17-1999 & 53.02 & & & 03-14-2000 & 55.60 & \\
\hline & & 11-01-1999 & 64.61 & & & & & \\
\hline & & 03-15-2000 & 55.23 & (C-36-11)8aab-1 & 5,563 & 09-14-1935 & 58.27 & \\
\hline & & 03-09-2001 & 60.00 & & & $10-14-1935$ & 59.39 & \\
\hline & & & & & & $11-25-1935$ & 57.00 & \\
\hline \multirow[t]{7}{*}{ (C-35-12)36caa-2 } & $5,504.7$ & 03-09-2001 & 22.61 & & & 01-30-1936 & 53.02 & \\
\hline & & 03-14-2001 & 20.66 & & & 04-18-1936 & 51.61 & \\
\hline & & $05-10-2001$ & 18.56 & & & 06-16-1936 & 58.26 & \\
\hline & & $05-11-2001$ & 18.80 & & & 08-02-1936 & 60.13 & \\
\hline & & $05-17-2001$ & 19.25 & & & 09-25-1936 & 62.65 & \\
\hline & & $05-23-2001$ & 19.08 & & & $11-23-1936$ & 58.30 & \\
\hline & & $05-24-2001$ & 19.43 & & & $12-08-1936$ & 58.30 & \\
\hline
\end{tabular}


Table 2. Water levels in selected wells in Cedar Valley, Iron County, southwestern Utah—Continued

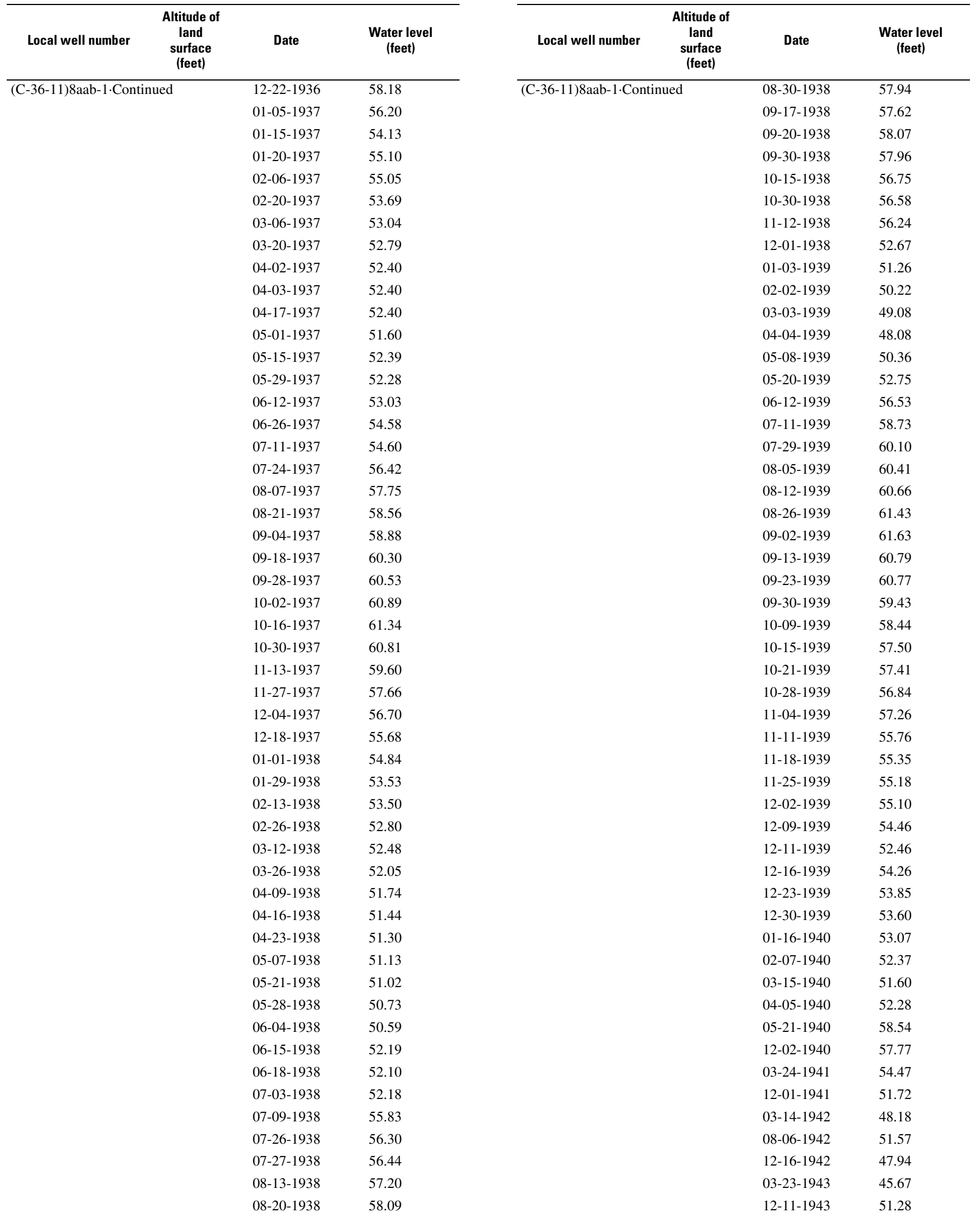


Table 2. Water levels in selected wells in Cedar Valley, Iron County, southwestern Utah-Continued

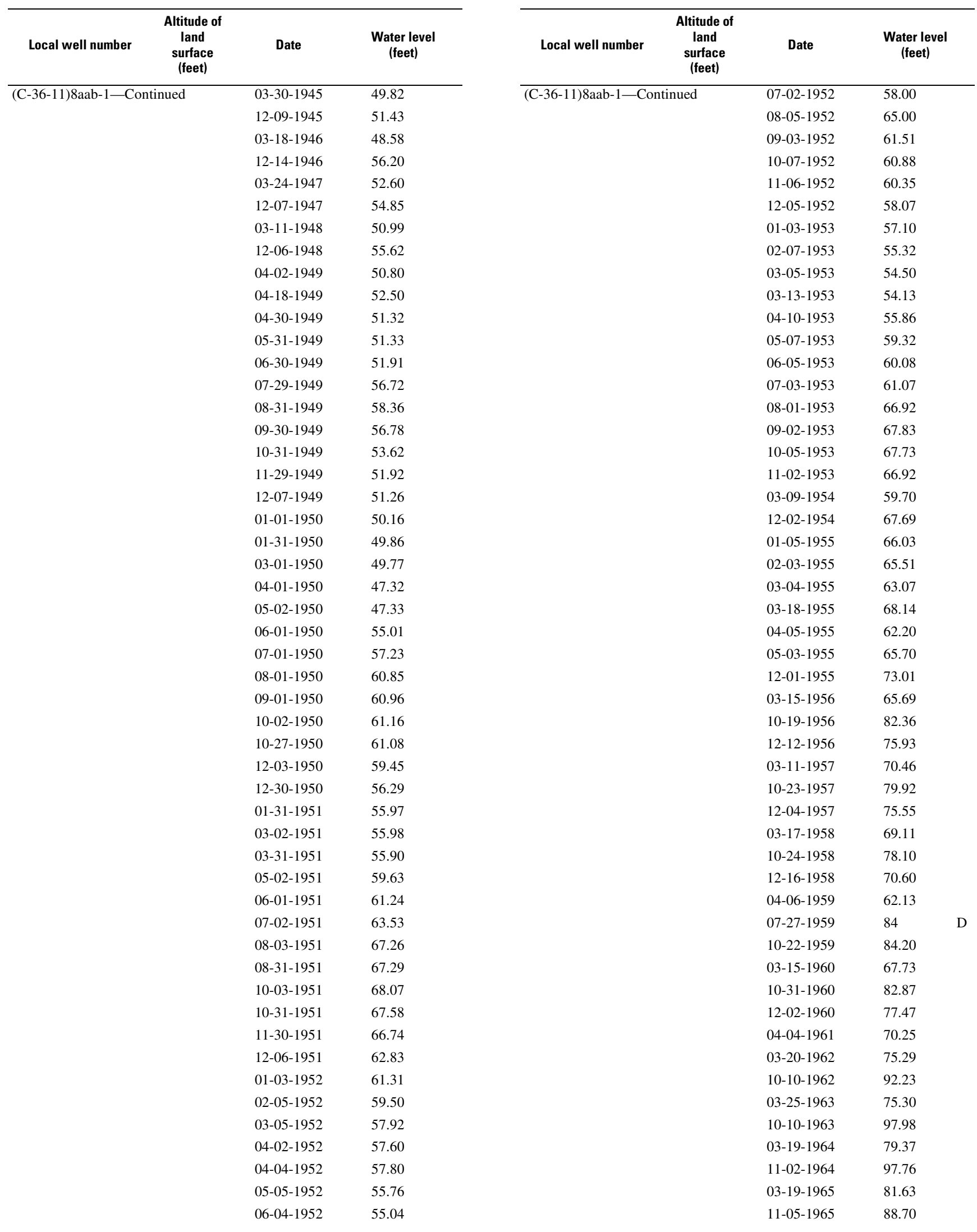


Table 2. Water levels in selected wells in Cedar Valley, Iron County, southwestern Utah-Continued

\begin{tabular}{|c|c|c|c|c|c|c|}
\hline Local well number & $\begin{array}{l}\text { Altitude of } \\
\text { land } \\
\text { surface } \\
\text { (feet) }\end{array}$ & Date & $\begin{array}{l}\text { Water level } \\
\text { (feet) }\end{array}$ & $\begin{array}{l}\text { Altitude of } \\
\text { land } \\
\text { surface } \\
\text { (feet) }\end{array}$ & Date & $\begin{array}{c}\text { Water level } \\
\text { (feet) }\end{array}$ \\
\hline \multirow{51}{*}{\multicolumn{2}{|c|}{ (C-36-11)8aab-1-Continued }} & 03-07-1966 & 77.78 & (C-36-11)8aab-1—Continued & $01-20-1979$ & 81.90 \\
\hline & & 09-27-1966 & 98.89 & & 01-25-1979 & 81.23 \\
\hline & & 03-06-1967 & 79.40 & & 01-31-1979 & 80.80 \\
\hline & & $10-06-1967$ & 90.10 & & 02-05-1979 & 80.40 \\
\hline & & 03-12-1968 & 72.40 & & 02-10-1979 & 80.10 \\
\hline & & $10-08-1968$ & 89.15 & & 02-15-1979 & 79.70 \\
\hline & & 03-17-1969 & 70.40 & & 02-20-1979 & 79.30 \\
\hline & & 03-11-1970 & 64.40 & & 02-25-1979 & 79.00 \\
\hline & & $10-08-1970$ & 87.90 & & 02-28-1979 & 78.73 \\
\hline & & 03-08-1971 & 71.47 & & 03-05-1979 & 78.48 \\
\hline & & $10-04-1971$ & 93.60 & & 03-10-1979 & 78.30 \\
\hline & & 03-08-1972 & 72.25 & & 03-15-1979 & 77.69 \\
\hline & & $10-04-1972$ & 92.20 & & 03-20-1979 & 77.39 \\
\hline & & 03-15-1973 & 68.58 & & 03-25-1979 & 77.11 \\
\hline & & 04-25-1978 & 79.52 & & 03-31-1979 & 76.80 \\
\hline & & 05-05-1978 & 79.40 & & 04-05-1979 & 76.44 \\
\hline & & 05-10-1978 & 79.95 & & 04-10-1979 & 76.00 \\
\hline & & 05-15-1978 & 80.10 & & 04-15-1979 & 75.85 \\
\hline & & 05-20-1978 & 80.99 & & 04-20-1979 & 75.58 \\
\hline & & 05-25-1978 & 80.49 & & 04-25-1979 & 76.18 \\
\hline & & $06-25-1978$ & 85.38 & & 04-30-1979 & 76.63 \\
\hline & & $06-30-1978$ & 86.94 & & 05-05-1979 & 76.22 \\
\hline & & 07-05-1978 & 89.28 & & 05-10-1979 & 76.92 \\
\hline & & 07-10-1978 & 90.16 & & 05-15-1979 & 77.27 \\
\hline & & 07-15-1978 & 91.76 & & 05-20-1979 & 76.77 \\
\hline & & 09-10-1978 & 100.08 & & $05-25-1979$ & 75.88 \\
\hline & & 09-15-1978 & 98.68 & & 05-31-1979 & 75.20 \\
\hline & & 09-20-1978 & 96.63 & & 06-05-1979 & 75.26 \\
\hline & & 09-25-1978 & 95.31 & & 06-10-1979 & 74.30 \\
\hline & & 09-30-1978 & 95.81 & & 06-15-1979 & 73.71 \\
\hline & & $10-05-1978$ & 96.40 & & 06-20-1979 & 73.54 \\
\hline & & $10-10-1978$ & 96.62 & & 06-25-1979 & 75.81 \\
\hline & & $10-15-1978$ & 95.68 & & 06-30-1979 & 77.14 \\
\hline & & $10-20-1978$ & 94.97 & & 07-05-1979 & 79.41 \\
\hline & & $10-25-1978$ & 93.60 & & 07-10-1979 & 80.78 \\
\hline & & $10-31-1978$ & 92.37 & & 07-15-1979 & 81.87 \\
\hline & & $11-05-1978$ & 91.48 & & 07-20-1979 & 83.21 \\
\hline & & $11-10-1978$ & 90.51 & & 07-25-1979 & 83.56 \\
\hline & & $11-15-1978$ & 89.81 & & 07-31-1979 & 83.41 \\
\hline & & $11-20-1978$ & 88.90 & & 08-05-1979 & 84.89 \\
\hline & & $11-25-1978$ & 88.10 & & 08-10-1979 & 85.84 \\
\hline & & $11-30-1978$ & 87.40 & & 08-15-1979 & 86.44 \\
\hline & & $12-05-1978$ & 86.70 & & 08-20-1979 & 86.50 \\
\hline & & $12-10-1978$ & 86.00 & & 08-25-1979 & 86.80 \\
\hline & & $12-15-1978$ & 85.50 & & 08-31-1979 & 87.10 \\
\hline & & $12-20-1978$ & 85.00 & & 09-05-1979 & 87.40 \\
\hline & & $12-25-1978$ & 84.60 & & 09-10-1979 & 87.70 \\
\hline & & $12-31-1978$ & 83.90 & & 09-15-1979 & 88.00 \\
\hline & & 01-05-1979 & 83.40 & & 09-20-1979 & 88.20 \\
\hline & & 01-10-1979 & 82.90 & & 09-25-1979 & 88.54 \\
\hline & & 01-15-1979 & 82.40 & & 09-30-1979 & 89.90 \\
\hline
\end{tabular}


Table 2. Water levels in selected wells in Cedar Valley, Iron County, southwestern Utah-Continued

\begin{tabular}{|c|c|c|c|c|c|}
\hline $\begin{array}{c}\text { Altitude of } \\
\text { land } \\
\text { surface } \\
\text { (feet) }\end{array}$ & Date & $\begin{array}{c}\text { Water level } \\
\text { (feet) }\end{array}$ & $\begin{array}{l}\text { Altitude of } \\
\text { land } \\
\text { surface } \\
\text { (feet) }\end{array}$ & Date & $\begin{array}{c}\text { Water level } \\
\text { (feet) }\end{array}$ \\
\hline \multirow[t]{51}{*}{ (C-36-11)8aab-1-Continued } & $10-05-1979$ & 89.65 & (C-36-11)8aab-1-Continued & $06-20-1980$ & 69.97 \\
\hline & $10-10-1979$ & 89.87 & & $06-25-1980$ & 70.31 \\
\hline & $10-15-1979$ & 89.54 & & 06-30-1980 & 70.96 \\
\hline & $10-20-1979$ & 87.93 & & 07-05-1980 & 71.79 \\
\hline & $10-25-1979$ & 86.18 & & $07-10-1980$ & 71.95 \\
\hline & $10-31-1979$ & 84.69 & & $07-15-1980$ & 72.65 \\
\hline & 11-05-1979 & 83.78 & & $07-20-1980$ & 73.35 \\
\hline & 11-10-1979 & 82.78 & & $07-25-1980$ & 74.06 \\
\hline & 11-15-1979 & 82.11 & & $07-31-1980$ & 74.76 \\
\hline & $11-20-1979$ & 81.16 & & 08-05-1980 & 75.46 \\
\hline & $11-25-1979$ & 81.27 & & $08-10-1980$ & 76.17 \\
\hline & $11-30-1979$ & 80.49 & & $08-15-1980$ & 76.88 \\
\hline & 12-05-1979 & 79.70 & & 08-20-1980 & 77.84 \\
\hline & $12-10-1979$ & 78.91 & & $08-25-1980$ & 78.88 \\
\hline & $12-15-1979$ & 77.88 & & $08-31-1980$ & 79.84 \\
\hline & $12-20-1979$ & 77.14 & & 09-05-1980 & 79.91 \\
\hline & $12-25-1979$ & 76.98 & & 09-10-1980 & 79.44 \\
\hline & $12-31-1979$ & 76.79 & & 09-15-1980 & 77.82 \\
\hline & 01-05-1980 & 76.65 & & 09-20-1980 & 77.51 \\
\hline & 01-10-1980 & 75.19 & & 09-25-1980 & 77.19 \\
\hline & 01-15-1980 & 73.72 & & 09-30-1980 & 76.88 \\
\hline & 01-20-1980 & 73.31 & & $10-01-1980$ & 77.88 \\
\hline & $01-25-1980$ & 72.79 & & $10-05-1980$ & 76.63 \\
\hline & 01-31-1980 & 71.36 & & $10-10-1980$ & 76.30 \\
\hline & 02-05-1980 & 68.48 & & $10-15-1980$ & 75.02 \\
\hline & 02-10-1980 & 68.63 & & $10-20-1980$ & 74.63 \\
\hline & $02-15-1980$ & 68.78 & & $10-25-1980$ & 73.08 \\
\hline & $02-20-1980$ & 68.93 & & $10-31-1980$ & 72.31 \\
\hline & $02-25-1980$ & 69.09 & & $11-05-1980$ & 71.50 \\
\hline & $02-29-1980$ & 69.22 & & $11-10-1980$ & 70.76 \\
\hline & 03-05-1980 & 69.39 & & $11-15-1980$ & 70.22 \\
\hline & $03-10-1980$ & 69.54 & & $11-20-1980$ & 69.54 \\
\hline & 03-15-1980 & 69.28 & & $11-25-1980$ & 68.94 \\
\hline & 03-20-1980 & 68.95 & & $11-30-1980$ & 68.31 \\
\hline & $03-25-1980$ & 68.82 & & $12-05-1980$ & 67.80 \\
\hline & 03-31-1980 & 68.59 & & $12-10-1980$ & 67.45 \\
\hline & 04-05-1980 & 68.37 & & $12-15-1980$ & 67.09 \\
\hline & 04-10-1980 & 68.12 & & $12-20-1980$ & 66.73 \\
\hline & $04-15-1980$ & 68.01 & & $12-25-1980$ & 66.37 \\
\hline & 04-20-1980 & 67.68 & & $12-31-1980$ & 65.94 \\
\hline & 04-25-1980 & 67.57 & & 01-05-1981 & 65.58 \\
\hline & $04-30-1980$ & 67.34 & & 01-10-1981 & 65.22 \\
\hline & 05-05-1980 & 67.45 & & 01-15-1981 & 64.86 \\
\hline & $05-10-1980$ & 67.35 & & 01-20-1981 & 64.48 \\
\hline & 05-15-1980 & 67.21 & & 01-25-1981 & 64.20 \\
\hline & 05-20-1980 & 67.03 & & 01-31-1981 & 63.90 \\
\hline & $05-25-1980$ & 66.66 & & 02-05-1981 & 63.60 \\
\hline & 05-31-1980 & 66.73 & & 02-10-1981 & 63.30 \\
\hline & 06-05-1980 & 67.42 & & 02-15-1981 & 62.80 \\
\hline & 06-10-1980 & 68.27 & & 02-20-1981 & 62.80 \\
\hline & $06-15-1980$ & 68.70 & & 02-25-1981 & 62.60 \\
\hline
\end{tabular}


Table 2. Water levels in selected wells in Cedar Valley, Iron County, southwestern Utah-Continued

\begin{tabular}{|c|c|c|c|c|c|c|c|}
\hline Local well number & $\begin{array}{c}\text { Altitude of } \\
\text { land } \\
\text { surface } \\
\text { (feet) }\end{array}$ & Date & $\begin{array}{l}\text { Water level } \\
\text { (feet) }\end{array}$ & Local well number & $\begin{array}{l}\text { Altitude of } \\
\text { land } \\
\text { surface } \\
\text { (feet) }\end{array}$ & Date & $\begin{array}{l}\text { Water level } \\
\text { (feet) }\end{array}$ \\
\hline \multirow{51}{*}{\multicolumn{2}{|c|}{ (C-36-11)8aab-1-Continued }} & $02-28-1981$ & 62.30 & \multirow{51}{*}{\multicolumn{2}{|c|}{ (C-36-11)8aab-1-Continued }} & $11-10-1981$ & 71.25 \\
\hline & & 03-03-1981 & 62.03 & & & $11-15-1981$ & 70.74 \\
\hline & & 03-05-1981 & 62.02 & & & 11-20-1981 & 70.21 \\
\hline & & 03-10-1981 & 61.96 & & & $11-25-1981$ & 69.62 \\
\hline & & 03-15-1981 & 61.73 & & & $11-30-1981$ & 69.30 \\
\hline & & 03-20-1981 & 61.33 & & & $12-05-1981$ & 68.80 \\
\hline & & 03-25-1981 & 61.25 & & & $12-10-1981$ & 68.35 \\
\hline & & 03-31-1981 & 61.17 & & & $12-15-1981$ & 67.93 \\
\hline & & 04-05-1981 & 61.08 & & & $12-20-1981$ & 67.53 \\
\hline & & 04-10-1981 & 61.00 & & & $12-25-1981$ & 67.19 \\
\hline & & 04-15-1981 & 60.88 & & & $12-31-1981$ & 66.70 \\
\hline & & 04-20-1981 & 60.82 & & & 01-05-1982 & 66.31 \\
\hline & & 04-25-1981 & 60.76 & & & 01-10-1982 & 66.16 \\
\hline & & 04-30-1981 & 61.33 & & & 01-15-1982 & 65.91 \\
\hline & & 05-05-1981 & 61.33 & & & 01-20-1982 & 65.44 \\
\hline & & 05-10-1981 & 64.70 & & & $01-25-1982$ & 65.37 \\
\hline & & 05-15-1981 & 65.84 & & & 01-31-1982 & 64.99 \\
\hline & & 05-20-1981 & 67.11 & & & 02-05-1982 & 65.02 \\
\hline & & 05-25-1981 & 67.84 & & & 02-10-1982 & 64.51 \\
\hline & & 05-31-1981 & 68.15 & & & 02-15-1982 & 64.42 \\
\hline & & 06-05-1981 & 69.07 & & & $02-20-1982$ & 64.20 \\
\hline & & 06-10-1981 & 69.60 & & & $02-25-1982$ & 63.98 \\
\hline & & 06-15-1981 & 71.19 & & & $02-28-1982$ & 63.87 \\
\hline & & 06-20-1981 & 72.88 & & & 03-01-1982 & 63.66 \\
\hline & & $06-25-1981$ & 74.23 & & & 03-05-1982 & 63.58 \\
\hline & & 06-30-1981 & 75.42 & & & 03-10-1982 & 63.23 \\
\hline & & 07-05-1981 & 76.43 & & & 03-15-1982 & 62.86 \\
\hline & & 07-10-1981 & 77.42 & & & 03-20-1982 & 62.75 \\
\hline & & 07-15-1981 & 78.07 & & & 03-25-1982 & 62.42 \\
\hline & & $07-20-1981$ & 78.92 & & & 03-31-1982 & 62.18 \\
\hline & & 07-25-1981 & 79.39 & & & 04-05-1982 & 61.99 \\
\hline & & 07-31-1981 & 80.30 & & & 04-10-1982 & 62.01 \\
\hline & & 08-05-1981 & 81.22 & & & 04-15-1982 & 61.82 \\
\hline & & 08-10-1981 & 82.04 & & & 04-20-1982 & 61.82 \\
\hline & & 08-15-1981 & 81.95 & & & 04-25-1982 & 61.48 \\
\hline & & 08-20-1981 & 81.90 & & & 04-30-1982 & 61.49 \\
\hline & & 08-25-1981 & 81.85 & & & 05-05-1982 & 61.50 \\
\hline & & 08-31-1981 & 81.84 & & & 05-10-1982 & 62.14 \\
\hline & & 09-05-1981 & 81.55 & & & $05-15-1982$ & 62.90 \\
\hline & & 09-10-1981 & 81.16 & & & 05-20-1982 & 64.78 \\
\hline & & 09-15-1981 & 79.33 & & & 05-25-1982 & 63.70 \\
\hline & & 09-20-1981 & 77.67 & & & 05-31-1982 & 66.07 \\
\hline & & 09-25-1981 & 78.05 & & & 06-05-1982 & 67.70 \\
\hline & & 09-30-1981 & 77.25 & & & 06-10-1982 & 68.51 \\
\hline & & $10-05-1981$ & 76.47 & & & 06-15-1982 & 69.28 \\
\hline & & $10-10-1981$ & 75.63 & & & $06-20-1982$ & 69.71 \\
\hline & & $10-15-1981$ & 74.65 & & & $06-25-1982$ & 70.97 \\
\hline & & $10-20-1981$ & 73.79 & & & $06-30-1982$ & 72.44 \\
\hline & & $10-25-1981$ & 73.14 & & & 07-05-1982 & 73.94 \\
\hline & & $10-31-1981$ & 72.53 & & & 07-10-1982 & 75.26 \\
\hline & & $11-05-1981$ & 71.81 & & & 07-15-1982 & 76.04 \\
\hline
\end{tabular}


Table 2. Water levels in selected wells in Cedar Valley, Iron County, southwestern Utah-Continued

\begin{tabular}{|c|c|c|c|c|c|c|c|}
\hline Local well number & $\begin{array}{l}\text { Altitude of } \\
\text { land } \\
\text { surface } \\
\text { (feet) }\end{array}$ & Date & $\begin{array}{c}\text { Water level } \\
\text { (feet) }\end{array}$ & Local well number & $\begin{array}{l}\text { Altitude of } \\
\text { land } \\
\text { surface } \\
\text { (feet) }\end{array}$ & Date & $\begin{array}{l}\text { Water level } \\
\text { (feet) }\end{array}$ \\
\hline (C-36-11)8aab-1-Co & inued & $07-20-1982$ & 76.99 & (C-36-11)8aab-1-C & inued & 04-05-1983 & 58.98 \\
\hline & & $07-25-1982$ & 77.63 & & & 04-10-1983 & 58.71 \\
\hline & & 07-31-1982 & 76.20 & & & 04-15-1983 & 58.78 \\
\hline & & 08-05-1982 & 76.50 & & & 04-20-1983 & 58.49 \\
\hline & & 08-10-1982 & 76.82 & & & $04-25-1983$ & 58.34 \\
\hline & & 08-15-1982 & 78.63 & & & 04-30-1983 & 58.19 \\
\hline & & 08-20-1982 & 79.55 & & & 05-05-1983 & 57.96 \\
\hline & & $08-25-1982$ & 79.30 & & & 05-10-1983 & 57.74 \\
\hline & & 08-31-1982 & 80.35 & & & $05-15-1983$ & 57.58 \\
\hline & & 09-05-1982 & 81.26 & & & $05-20-1983$ & 58.46 \\
\hline & & 09-10-1982 & 81.37 & & & $05-25-1983$ & 57.76 \\
\hline & & 09-15-1982 & 78.26 & & & $05-31-1983$ & 57.50 \\
\hline & & 09-20-1982 & 77.38 & & & 06-05-1983 & 57.46 \\
\hline & & 09-25-1982 & 76.62 & & & 06-10-1983 & 57.40 \\
\hline & & 09-30-1982 & 75.19 & & & $06-15-1983$ & 57.35 \\
\hline & & $10-05-1982$ & 74.06 & & & $06-20-1983$ & 57.29 \\
\hline & & $10-10-1982$ & 73.05 & & & $06-25-1983$ & 57.26 \\
\hline & & $10-15-1982$ & 72.68 & & & 06-30-1983 & 57.23 \\
\hline & & $10-20-1982$ & 72.30 & & & 07-05-1983 & 57.21 \\
\hline & & $10-25-1982$ & 71.24 & & & 07-10-1983 & 57.18 \\
\hline & & $10-31-1982$ & 70.46 & & & $07-15-1983$ & 57.16 \\
\hline & & 11-05-1982 & 69.83 & & & 07-20-1983 & 57.12 \\
\hline & & $11-10-1982$ & 69.19 & & & $07-25-1983$ & 57.08 \\
\hline & & $11-15-1982$ & 68.81 & & & 07-31-1983 & 57.04 \\
\hline & & $11-20-1982$ & 68.35 & & & 08-05-1983 & 57.01 \\
\hline & & $11-25-1982$ & 68.01 & & & $08-10-1983$ & 56.68 \\
\hline & & $11-30-1982$ & 67.12 & & & $08-15-1983$ & 55.77 \\
\hline & & $12-05-1982$ & 67.08 & & & $08-20-1983$ & 55.08 \\
\hline & & $12-10-1982$ & 66.71 & & & 08-25-1983 & 54.77 \\
\hline & & $12-15-1982$ & 66.46 & & & 08-31-1983 & 56.31 \\
\hline & & $12-20-1982$ & 65.97 & & & 09-05-1983 & 57.96 \\
\hline & & $12-25-1982$ & 65.75 & & & 09-10-1983 & 60.53 \\
\hline & & $12-31-1982$ & 65.28 & & & 09-15-1983 & 61.40 \\
\hline & & 01-05-1983 & 64.90 & & & 09-20-1983 & 60.01 \\
\hline & & 01-10-1983 & 64.55 & & & $09-25-1983$ & 58.94 \\
\hline & & 01-15-1983 & 64.10 & & & 09-30-1983 & 57.86 \\
\hline & & 01-20-1983 & 63.76 & & & $10-05-1983$ & 57.36 \\
\hline & & 01-25-1983 & 63.56 & & & $10-10-1983$ & 56.87 \\
\hline & & 01-31-1983 & 63.03 & & & $10-15-1983$ & 56.36 \\
\hline & & 02-05-1983 & 62.70 & & & $10-20-1983$ & 56.16 \\
\hline & & 02-10-1983 & 62.30 & & & $10-25-1983$ & 55.82 \\
\hline & & 02-15-1983 & 61.89 & & & $10-31-1983$ & 55.28 \\
\hline & & 02-20-1983 & 61.61 & & & $11-05-1983$ & 54.98 \\
\hline & & $02-25-1983$ & 61.12 & & & $11-10-1983$ & 54.47 \\
\hline & & 02-28-1983 & 60.95 & & & $11-15-1983$ & 54.29 \\
\hline & & 03-05-1983 & 60.63 & & & $11-20-1983$ & 53.98 \\
\hline & & 03-10-1983 & 60.16 & & & $11-25-1983$ & 53.50 \\
\hline & & $03-15-1983$ & 59.96 & & & $11-30-1983$ & 53.30 \\
\hline & & 03-20-1983 & 59.64 & & & $12-05-1983$ & 53.10 \\
\hline & & 03-25-1983 & 59.37 & & & $12-10-1983$ & 52.90 \\
\hline & & $03-31-1983$ & 59.04 & & & $12-15-1983$ & 52.70 \\
\hline
\end{tabular}


Table 2. Water levels in selected wells in Cedar Valley, Iron County, southwestern Utah-Continued

\begin{tabular}{|c|c|c|c|c|c|c|c|}
\hline Local well number & $\begin{array}{l}\text { Altitude of } \\
\text { land } \\
\text { surface } \\
\text { (feet) }\end{array}$ & Date & $\begin{array}{c}\text { Water level } \\
\text { (feet) }\end{array}$ & Local well number & $\begin{array}{c}\text { Altitude of } \\
\text { land } \\
\text { surface } \\
\text { (feet) }\end{array}$ & Date & $\begin{array}{c}\text { Water level } \\
\text { (feet) }\end{array}$ \\
\hline \multirow{51}{*}{\multicolumn{2}{|c|}{ (C-36-11)8aab-1-Continued }} & $12-20-1983$ & 52.48 & \multirow{51}{*}{\multicolumn{2}{|c|}{ (C-36-11)8aab-1-Continued }} & $\begin{array}{l}09-05-1984 \\
\end{array}$ & 59.12 \\
\hline & & $12-25-1983$ & 52.37 & & & 09-10-1984 & 59.23 \\
\hline & & $12-31-1983$ & 52.34 & & & 09-15-1984 & 58.51 \\
\hline & & 01-05-1984 & 52.07 & & & 09-20-1984 & 57.60 \\
\hline & & 01-10-1984 & 51.76 & & & 09-25-1984 & 57.31 \\
\hline & & 01-15-1984 & 51.83 & & & 09-30-1984 & 56.74 \\
\hline & & 01-20-1984 & 51.52 & & & $10-05-1984$ & 56.28 \\
\hline & & 01-25-1984 & 51.48 & & & $10-10-1984$ & 55.61 \\
\hline & & 01-31-1984 & 51.18 & & & $10-15-1984$ & 55.05 \\
\hline & & 02-05-1984 & 51.03 & & & $10-20-1984$ & 54.70 \\
\hline & & 02-10-1984 & 50.64 & & & $10-25-1984$ & 54.48 \\
\hline & & 02-15-1984 & 50.65 & & & $10-31-1984$ & 54.03 \\
\hline & & 02-20-1984 & 50.42 & & & 11-05-1984 & 53.76 \\
\hline & & 02-25-1984 & 50.03 & & & 11-10-1984 & 53.55 \\
\hline & & 02-29-1984 & 49.95 & & & 11-15-1984 & 53.25 \\
\hline & & 03-05-1984 & 49.79 & & & 11-20-1984 & 52.99 \\
\hline & & 03-10-1984 & 49.58 & & & $11-25-1984$ & 52.62 \\
\hline & & 03-15-1984 & 49.54 & & & $11-30-1984$ & 52.57 \\
\hline & & 03-20-1984 & 49.26 & & & $12-05-1984$ & 52.43 \\
\hline & & 03-25-1984 & 49.19 & & & $12-10-1984$ & 52.09 \\
\hline & & 03-31-1984 & 48.97 & & & $12-15-1984$ & 51.87 \\
\hline & & 04-05-1984 & 48.96 & & & $12-20-1984$ & 51.72 \\
\hline & & 04-10-1984 & 48.85 & & & $12-25-1984$ & 51.58 \\
\hline & & 04-15-1984 & 48.99 & & & $12-31-1984$ & 51.48 \\
\hline & & 04-20-1984 & 48.78 & & & 01-05-1985 & 51.24 \\
\hline & & 04-25-1984 & 48.53 & & & 01-10-1985 & 51.15 \\
\hline & & 04-30-1984 & 48.75 & & & 01-15-1985 & 50.87 \\
\hline & & 05-05-1984 & 48.94 & & & 01-20-1985 & 50.66 \\
\hline & & 05-10-1984 & 49.78 & & & 01-25-1985 & 50.55 \\
\hline & & 05-15-1984 & 50.47 & & & 01-31-1985 & 50.22 \\
\hline & & 05-20-1984 & 50.06 & & & 02-05-1985 & 50.06 \\
\hline & & 05-25-1984 & 50.19 & & & 02-10-1985 & 50.07 \\
\hline & & 05-31-1984 & 52.39 & & & 02-15-1985 & 49.64 \\
\hline & & 06-05-1984 & 51.99 & & & 02-20-1985 & 49.22 \\
\hline & & 06-10-1984 & 52.56 & & & 02-25-1985 & 49.30 \\
\hline & & 06-15-1984 & 53.45 & & & 02-28-1985 & 49.21 \\
\hline & & 06-20-1984 & 54.34 & & & 03-05-1985 & 48.89 \\
\hline & & 06-25-1984 & 55.86 & & & 03-10-1985 & 48.84 \\
\hline & & 06-30-1984 & 57.72 & & & 03-15-1985 & 48.68 \\
\hline & & 07-05-1984 & 58.78 & & & 03-20-1985 & 48.57 \\
\hline & & 07-10-1984 & 59.38 & & & 03-25-1985 & 48.30 \\
\hline & & 07-15-1984 & 59.37 & & & 03-31-1985 & 48.51 \\
\hline & & 07-20-1984 & 59.37 & & & 04-05-1985 & 48.27 \\
\hline & & 07-25-1984 & 57.51 & & & 04-10-1985 & 48.17 \\
\hline & & 07-31-1984 & 56.79 & & & 04-15-1985 & 48.04 \\
\hline & & 08-05-1984 & 55.80 & & & 04-20-1985 & 47.88 \\
\hline & & 08-10-1984 & 58.25 & & & 04-25-1985 & 47.69 \\
\hline & & 08-15-1984 & 58.48 & & & 04-30-1985 & 47.70 \\
\hline & & 08-20-1984 & 58.31 & & & 05-05-1985 & 47.45 \\
\hline & & 08-25-1984 & 56.70 & & & 05-10-1985 & 47.14 \\
\hline & & 08-31-1984 & 57.98 & & & 05-15-1985 & 47.08 \\
\hline
\end{tabular}


Table 2. Water levels in selected wells in Cedar Valley, Iron County, southwestern Utah-Continued

\begin{tabular}{|c|c|c|c|c|c|c|c|}
\hline Local well number & $\begin{array}{c}\text { Altitude of } \\
\text { land } \\
\text { surface } \\
\text { (feet) }\end{array}$ & Date & $\begin{array}{c}\text { Water level } \\
\text { (feet) }\end{array}$ & Local well number & $\begin{array}{l}\text { Altitude of } \\
\text { land } \\
\text { surface } \\
\text { (feet) }\end{array}$ & Date & $\begin{array}{l}\text { Water level } \\
\text { (feet) }\end{array}$ \\
\hline \multirow{51}{*}{\multicolumn{2}{|c|}{ (C-36-11)8aab-1-Continued }} & 05-20-1985 & 46.98 & (C-36-11)8aab-1-Cc & inued & $02-05-1986$ & 50.51 \\
\hline & & 05-25-1985 & 47.07 & & & 02-10-1986 & 50.38 \\
\hline & & 05-31-1985 & 48.46 & & & 02-15-1986 & 50.24 \\
\hline & & 06-05-1985 & 49.70 & & & 02-20-1986 & 50.11 \\
\hline & & 06-10-1985 & 49.99 & & & $02-25-1986$ & 49.98 \\
\hline & & 06-15-1985 & 51.53 & & & 02-28-1986 & 49.91 \\
\hline & & 06-20-1985 & 52.95 & & & 03-05-1986 & 49.78 \\
\hline & & $06-25-1985$ & 53.95 & & & 03-10-1986 & 49.64 \\
\hline & & 06-30-1985 & 55.54 & & & 03-15-1986 & 49.51 \\
\hline & & 07-05-1985 & 56.84 & & & 03-20-1986 & 49.37 \\
\hline & & 07-10-1985 & 57.96 & & & 03-25-1986 & 49.23 \\
\hline & & 07-15-1985 & 59.36 & & & 03-31-1986 & 49.05 \\
\hline & & 07-20-1985 & 58.93 & & & 04-05-1986 & 48.93 \\
\hline & & 07-25-1985 & 55.73 & & & 04-10-1986 & 48.78 \\
\hline & & 07-31-1985 & 56.10 & & & 04-15-1986 & 48.70 \\
\hline & & 08-05-1985 & 57.00 & & & 04-20-1986 & 48.72 \\
\hline & & 08-10-1985 & 58.50 & & & 04-25-1986 & 48.43 \\
\hline & & 08-15-1985 & 60.00 & & & 04-30-1986 & 48.71 \\
\hline & & 08-20-1985 & 60.85 & & & 05-05-1986 & 48.70 \\
\hline & & 08-25-1985 & 60.60 & & & 05-10-1986 & 49.12 \\
\hline & & 08-31-1985 & 60.30 & & & 05-15-1986 & 51.41 \\
\hline & & 09-05-1985 & 60.05 & & & 05-20-1986 & 51.89 \\
\hline & & 09-10-1985 & 59.80 & & & 05-25-1986 & 54.33 \\
\hline & & 09-15-1985 & 59.55 & & & 05-31-1986 & 55.64 \\
\hline & & 09-20-1985 & 58.77 & & & 06-05-1986 & 56.20 \\
\hline & & 09-25-1985 & 58.68 & & & $06-10-1986$ & 55.58 \\
\hline & & 09-30-1985 & 58.14 & & & $06-15-1986$ & 55.21 \\
\hline & & $10-05-1985$ & 57.23 & & & 06-20-1986 & 56.84 \\
\hline & & $10-10-1985$ & 56.57 & & & $06-25-1986$ & 57.70 \\
\hline & & $10-15-1985$ & 56.06 & & & 06-30-1986 & 59.44 \\
\hline & & $10-20-1985$ & 55.56 & & & 07-05-1986 & 59.97 \\
\hline & & $10-25-1985$ & 55.54 & & & 07-10-1986 & 60.82 \\
\hline & & $10-31-1985$ & 55.02 & & & 07-15-1986 & 60.48 \\
\hline & & 11-05-1985 & 54.70 & & & 07-20-1986 & 60.58 \\
\hline & & $11-10-1985$ & 54.26 & & & 07-25-1986 & 61.00 \\
\hline & & $11-15-1985$ & 54.06 & & & $07-31-1986$ & 60.66 \\
\hline & & 11-20-1985 & 53.59 & & & 08-05-1986 & 61.26 \\
\hline & & $11-25-1985$ & 53.40 & & & 08-10-1986 & 62.09 \\
\hline & & $11-30-1985$ & 53.07 & & & 08-15-1986 & 62.66 \\
\hline & & $12-05-1985$ & 52.94 & & & 08-20-1986 & 63.88 \\
\hline & & $12-10-1985$ & 52.71 & & & 08-25-1986 & 63.07 \\
\hline & & $12-15-1985$ & 52.62 & & & 08-31-1986 & 60.08 \\
\hline & & $12-20-1985$ & 52.42 & & & 09-05-1986 & 59.53 \\
\hline & & $12-25-1985$ & 52.17 & & & 09-10-1986 & 58.59 \\
\hline & & $12-31-1985$ & 51.92 & & & $09-15-1986$ & 57.83 \\
\hline & & 01-05-1986 & 51.70 & & & 09-20-1986 & 58.34 \\
\hline & & 01-10-1986 & 51.55 & & & 09-25-1986 & 57.43 \\
\hline & & 01-15-1986 & 51.26 & & & 09-30-1986 & 56.68 \\
\hline & & 01-20-1986 & 51.02 & & & $10-20-1986$ & 54.20 \\
\hline & & 01-25-1986 & 51.03 & & & $10-25-1986$ & 54.02 \\
\hline & & 01-31-1986 & 50.64 & & & $10-31-1986$ & 53.61 \\
\hline
\end{tabular}


Table 2. Water levels in selected wells in Cedar Valley, Iron County, southwestern Utah-Continued

\begin{tabular}{|c|c|c|c|c|c|c|c|}
\hline Local well number & $\begin{array}{c}\text { Altitude of } \\
\text { land } \\
\text { surface } \\
\text { (feet) }\end{array}$ & Date & $\begin{array}{c}\text { Water level } \\
\text { (feet) }\end{array}$ & Local well number & $\begin{array}{c}\text { Altitude of } \\
\text { land } \\
\text { surface } \\
\text { (feet) }\end{array}$ & Date & $\begin{array}{c}\text { Water level } \\
\text { (feet) }\end{array}$ \\
\hline$(\mathrm{C}-36-11) 8 \mathrm{aab}-1-\mathrm{Co}$ & inued & $11-05-1986$ & 53.33 & $\overline{(C-36-11) 8 a a b-1-C}$ & inued & 09-10-1987 & 65.67 \\
\hline & & 11-10-1986 & 53.31 & & & 09-20-1987 & 61.34 \\
\hline & & $11-15-1986$ & 53.22 & & & 09-25-1987 & 60.87 \\
\hline & & $11-20-1986$ & 53.10 & & & 09-30-1987 & 60.42 \\
\hline & & $11-25-1986$ & 52.90 & & & $10-05-1987$ & 60.12 \\
\hline & & $11-30-1986$ & 52.88 & & & $10-10-1987$ & 59.61 \\
\hline & & $12-05-1986$ & 52.73 & & & $10-15-1987$ & 59.33 \\
\hline & & $12-10-1986$ & 52.73 & & & $10-20-1987$ & 58.53 \\
\hline & & $12-15-1986$ & 52.65 & & & $10-25-1987$ & 58.22 \\
\hline & & $12-20-1986$ & 52.56 & & & $12-20-1987$ & 54.67 \\
\hline & & $12-25-1986$ & 52.45 & & & $12-25-1987$ & 54.40 \\
\hline & & $12-31-1986$ & 52.34 & & & $12-31-1987$ & 54.14 \\
\hline & & 01-05-1987 & 51.89 & & & 01-05-1988 & 53.82 \\
\hline & & 01-10-1987 & 52.15 & & & 01-10-1988 & 53.69 \\
\hline & & 01-15-1987 & 51.72 & & & 01-15-1988 & 53.36 \\
\hline & & 01-20-1987 & 51.75 & & & 01-20-1988 & 53.35 \\
\hline & & 01-25-1987 & 51.66 & & & 01-25-1988 & 53.20 \\
\hline & & 01-31-1987 & 50.39 & & & 01-31-1988 & 52.82 \\
\hline & & 02-05-1987 & 50.42 & & & 02-05-1988 & 52.81 \\
\hline & & 02-10-1987 & 51.18 & & & 02-10-1988 & 52.55 \\
\hline & & 02-15-1987 & 50.97 & & & 02-15-1988 & 52.25 \\
\hline & & 02-20-1987 & 50.96 & & & 02-20-1988 & 52.26 \\
\hline & & 02-25-1987 & 50.67 & & & $02-25-1988$ & 52.05 \\
\hline & & 03-05-1987 & 50.58 & & & 02-29-1988 & 51.86 \\
\hline & & 03-10-1987 & 50.44 & & & 03-05-1988 & 51.80 \\
\hline & & 03-15-1987 & 50.03 & & & 03-10-1988 & 51.58 \\
\hline & & 03-20-1987 & 50.00 & & & 03-15-1988 & 51.35 \\
\hline & & 03-25-1987 & 50.03 & & & 03-20-1988 & 51.40 \\
\hline & & 03-31-1987 & 49.97 & & & 03-25-1988 & 51.31 \\
\hline & & 04-05-1987 & 49.77 & & & 03-31-1988 & 51.11 \\
\hline & & 04-10-1987 & 49.67 & & & 04-05-1988 & 51.12 \\
\hline & & 04-15-1987 & 49.70 & & & 04-10-1988 & 51.24 \\
\hline & & 04-20-1987 & 49.70 & & & 04-15-1988 & 50.87 \\
\hline & & 04-25-1987 & 49.51 & & & 04-20-1988 & 50.69 \\
\hline & & 04-30-1987 & 49.58 & & & 04-25-1988 & 50.72 \\
\hline & & 05-05-1987 & 50.08 & & & 04-30-1988 & 50.32 \\
\hline & & 05-10-1987 & 50.78 & & & 05-05-1988 & 50.30 \\
\hline & & 05-31-1987 & 53.67 & & & 05-10-1988 & 50.50 \\
\hline & & 06-15-1987 & 55.77 & & & 05-15-1988 & 50.64 \\
\hline & & 06-25-1987 & 58.88 & & & 05-20-1988 & 50.24 \\
\hline & & 06-30-1987 & 59.96 & & & 05-25-1988 & 50.02 \\
\hline & & 07-05-1987 & 60.63 & & & 05-31-1988 & 49.60 \\
\hline & & 07-25-1987 & 59.70 & & & 06-05-1988 & 50.72 \\
\hline & & 07-31-1987 & 59.70 & & & 06-10-1988 & 50.99 \\
\hline & & 08-05-1987 & 59.82 & & & 06-15-1988 & 51.42 \\
\hline & & 08-10-1987 & 61.53 & & & 06-20-1988 & 51.53 \\
\hline & & 08-15-1987 & 62.06 & & & 06-25-1988 & 51.83 \\
\hline & & 08-20-1987 & 62.59 & & & 06-30-1988 & 53.25 \\
\hline & & 08-25-1987 & 62.94 & & & 07-05-1988 & 54.16 \\
\hline & & 08-31-1987 & 63.93 & & & 07-10-1988 & 55.81 \\
\hline & & 09-05-1987 & 64.76 & & & 07-15-1988 & 57.14 \\
\hline
\end{tabular}


Table 2. Water levels in selected wells in Cedar Valley, Iron County, southwestern Utah—Continued

\begin{tabular}{|c|c|c|c|c|c|c|}
\hline Local well number & $\begin{array}{c}\text { Altitude of } \\
\text { land } \\
\text { surface } \\
\text { (feet) }\end{array}$ & Date & $\begin{array}{c}\text { Water level } \\
\text { (feet) }\end{array}$ & $\begin{array}{c}\text { Altitude of } \\
\text { land } \\
\text { surface } \\
\text { (feet) }\end{array}$ & Date & $\begin{array}{c}\text { Water level } \\
\text { (feet) }\end{array}$ \\
\hline \multirow{51}{*}{\multicolumn{2}{|c|}{ (C-36-11)8aab-1-Continued }} & $07-20-1988$ & 57.67 & \multirow[t]{51}{*}{ (C-36-11)8aab-1-Continued } & 04-25-1999 & 71.47 \\
\hline & & 07-25-1988 & 58.25 & & 04-30-1999 & 71.87 \\
\hline & & 07-31-1988 & 59.05 & & 05-05-1999 & 72.03 \\
\hline & & 09-19-1988 & 62.30 & & 05-10-1999 & 73.00 \\
\hline & & 03-09-1989 & 50.06 & & 05-15-1999 & 73.68 \\
\hline & & 09-22-1989 & 72.12 & & 05-20-1999 & 74.52 \\
\hline & & 03-01-1990 & 61.13 & & 05-25-1999 & 75.64 \\
\hline & & 09-22-1990 & 87.91 & & 05-31-1999 & 79.61 \\
\hline & & 03-01-1991 & 78.17 & & 06-05-1999 & 81.15 \\
\hline & & 09-18-1991 & 94.88 & & 06-10-1999 & 81.96 \\
\hline & & 03-10-1992 & 73.15 & & 06-15-1999 & 83.94 \\
\hline & & 03-02-1993 & 75.58 & & 06-20-1999 & 85.28 \\
\hline & & 03-09-1994 & 67.76 & & 06-25-1999 & 86.94 \\
\hline & & 03-09-1995 & 77.56 & & 06-30-1999 & 88.31 \\
\hline & & 03-08-1996 & 66.96 & & 07-05-1999 & 89.54 \\
\hline & & 03-04-1997 & 75.97 & & 07-10-1999 & 90.69 \\
\hline & & 11-25-1997 & 84.48 & & 07-15-1999 & 91.47 \\
\hline & & 03-16-1998 & 77.06 & & 07-20-1999 & 92.64 \\
\hline & & 11-20-1998 & 80.61 & & 07-25-1999 & 91.97 \\
\hline & & $11-25-1998$ & 80.03 & & 07-31-1999 & 90.63 \\
\hline & & $11-30-1998$ & 79.36 & & 08-05-1999 & 91.60 \\
\hline & & 12-05-1998 & 78.47 & & 08-10-1999 & 92.83 \\
\hline & & $12-10-1998$ & 77.67 & & 08-15-1999 & 94.11 \\
\hline & & $12-15-1998$ & 77.08 & & 08-20-1999 & 95.20 \\
\hline & & $12-20-1998$ & 76.36 & & 08-25-1999 & 95.10 \\
\hline & & $12-25-1998$ & 76.03 & & 08-31-1999 & 95.10 \\
\hline & & $12-31-1998$ & 75.35 & & 09-05-1999 & 95.00 \\
\hline & & 01-05-1999 & 75.17 & & 09-10-1999 & 95.00 \\
\hline & & 01-10-1999 & 74.81 & & 09-15-1999 & 94.90 \\
\hline & & 01-15-1999 & 74.36 & & 09-20-1999 & 94.90 \\
\hline & & 01-20-1999 & 73.96 & & 09-25-1999 & 94.90 \\
\hline & & 01-25-1999 & 73.65 & & 09-30-1999 & 94.80 \\
\hline & & 01-31-1999 & 73.31 & & 10-05-1999 & 94.80 \\
\hline & & 02-05-1999 & 73.11 & & 10-10-1999 & 94.44 \\
\hline & & 02-10-1999 & 72.58 & & 10-15-1999 & 93.44 \\
\hline & & 02-15-1999 & 72.39 & & 10-20-1999 & 92.21 \\
\hline & & 02-20-1999 & 72.11 & & 10-25-1999 & 91.07 \\
\hline & & 02-25-1999 & 71.54 & & 10-31-1999 & 89.84 \\
\hline & & 02-28-1999 & 71.15 & & 11-05-1999 & 88.88 \\
\hline & & 03-05-1999 & 70.79 & & 11-10-1999 & 87.96 \\
\hline & & 03-10-1999 & 70.64 & & 11-15-1999 & 87.13 \\
\hline & & 03-15-1999 & 70.42 & & 11-20-1999 & 86.30 \\
\hline & & 03-16-1999 & 70.46 & & 11-25-1999 & 85.64 \\
\hline & & 03-17-1999 & 70.51 & & $11-30-1999$ & 84.87 \\
\hline & & 03-20-1999 & 70.30 & & 12-05-1999 & 84.28 \\
\hline & & 03-25-1999 & 70.29 & & $12-10-1999$ & 83.66 \\
\hline & & 03-31-1999 & 70.09 & & 12-15-1999 & 83.14 \\
\hline & & 04-05-1999 & 70.18 & & $12-20-1999$ & 82.55 \\
\hline & & 04-10-1999 & 70.16 & & $12-25-1999$ & 82.07 \\
\hline & & 04-15-1999 & 70.35 & & 12-31-1999 & 81.50 \\
\hline & & 04-20-1999 & 71.04 & & 01-05-2000 & 81.10 \\
\hline
\end{tabular}


Table 2. Water levels in selected wells in Cedar Valley, Iron County, southwestern Utah-Continued

\begin{tabular}{|c|c|c|c|c|c|c|c|c|}
\hline Local well number & $\begin{array}{l}\text { Altitude of } \\
\text { land } \\
\text { surface } \\
\text { (feet) }\end{array}$ & Date & $\begin{array}{c}\text { Water level } \\
\text { (feet) }\end{array}$ & Local well number & $\begin{array}{l}\text { Altitude of } \\
\text { land } \\
\text { surface } \\
\text { (feet) }\end{array}$ & Date & \multicolumn{2}{|l|}{$\begin{array}{c}\text { Water level } \\
\text { (feet) }\end{array}$} \\
\hline \multirow{51}{*}{\multicolumn{2}{|c|}{ (C-36-11)8aab-1-Continued }} & $01-10-2000$ & 80.60 & \multicolumn{2}{|c|}{ (C-36-11)8aab-1-Continued } & $09-25-2000$ & 104.30 & \\
\hline & & $01-15-2000$ & 80.12 & & & $09-30-2000$ & 104.34 & \\
\hline & & $01-20-2000$ & 79.63 & & & $10-04-2000$ & 104.43 & \\
\hline & & $01-25-2000$ & 79.19 & & & $11-03-2000$ & 97.22 & \\
\hline & & $01-31-2000$ & 78.82 & & & $12-07-2000$ & 91.06 & \\
\hline & & $02-05-2000$ & 78.44 & & & $12-29-2000$ & 88.42 & \\
\hline & & $02-10-2000$ & 77.92 & & & 01-24-2001 & 85.66 & \\
\hline & & $02-15-2000$ & 77.71 & & & 03-02-2001 & 82.48 & \\
\hline & & $02-20-2000$ & 77.28 & & & & & \\
\hline & & $02-25-2000$ & 77.08 & (C-36-11)8abd-1 & 5,562 & 10-09-1972 & 92 & D \\
\hline & & $02-29-2000$ & 76.69 & & & 05-21-1998 & 86.90 & \\
\hline & & 03-05-2000 & 76.30 & & & $11-19-1998$ & 85.70 & \\
\hline & & 03-10-2000 & 76.25 & & & 03-17-1999 & 76.65 & \\
\hline & & $03-15-2000$ & 75.84 & & & 11-02-1999 & 93.35 & \\
\hline & & 03-20-2000 & 75.48 & & & 03-14-2000 & 81.08 & \\
\hline & & $03-25-2000$ & 75.35 & & & & & \\
\hline & & 03-31-2000 & 75.02 & (C-36-11)8aca-1 & 5,563 & $12-07-1953$ & 64.29 & \\
\hline & & 04-05-2000 & 75.33 & & & $12-02-1954$ & 66.63 & \\
\hline & & $04-10-2000$ & 74.97 & & & 05-26-1955 & 67.54 & \\
\hline & & $04-15-2000$ & 75.02 & & & $12-01-1955$ & 69.30 & \\
\hline & & $04-20-2000$ & 75.19 & & & 03-15-1956 & 68.04 & \\
\hline & & $04-25-2000$ & 75.65 & & & 05-11-1956 & 68.64 & \\
\hline & & 04-30-2000 & 76.19 & & & 06-06-1956 & 70.20 & \\
\hline & & $05-05-2000$ & 77.14 & & & 07-03-1956 & 70.62 & \\
\hline & & $05-10-2000$ & 79.41 & & & 08-07-1956 & 71.56 & \\
\hline & & $05-15-2000$ & 81.49 & & & 09-04-1956 & 72.51 & \\
\hline & & $05-20-2000$ & 83.74 & & & $10-17-1956$ & 73.12 & \\
\hline & & $05-25-2000$ & 85.24 & & & $12-12-1956$ & 72.94 & \\
\hline & & $05-31-2000$ & 87.42 & & & 03-11-1957 & 71.88 & \\
\hline & & 06-05-2000 & 88.32 & & & 04-17-1957 & 71.87 & \\
\hline & & 06-10-2000 & 90.14 & & & 05-20-1957 & 72.43 & \\
\hline & & $06-15-2000$ & 91.61 & & & 06-14-1957 & 72.59 & \\
\hline & & $06-20-2000$ & 92.72 & & & 08-12-1957 & 73.96 & \\
\hline & & $06-25-2000$ & 92.40 & & & 09-10-1957 & 74.56 & \\
\hline & & $06-30-2000$ & 92.94 & & & $10-23-1957$ & 74.84 & \\
\hline & & 07-05-2000 & 94.22 & & & $12-04-1957$ & 74.11 & \\
\hline & & $07-10-2000$ & 96.07 & & & 03-17-1958 & 72.32 & \\
\hline & & $07-15-2000$ & 97.05 & & & 04-30-1958 & 71.77 & \\
\hline & & $07-20-2000$ & 98.00 & & & 06-16-1958 & 71.41 & \\
\hline & & $07-25-2000$ & 98.70 & & & 07-15-1958 & 71.82 & \\
\hline & & $07-31-2000$ & 99.82 & & & 08-25-1958 & 72.67 & \\
\hline & & $08-05-2000$ & 100.91 & & & 09-16-1958 & 73.00 & \\
\hline & & $08-10-2000$ & 101.63 & & & $10-24-1958$ & 73.62 & \\
\hline & & $08-15-2000$ & 102.71 & & & $12-16-1958$ & 72.20 & \\
\hline & & $08-20-2000$ & 103.40 & & & 04-08-1959 & 70.58 & \\
\hline & & $08-25-2000$ & 103.97 & & & 06-01-1959 & 73.01 & \\
\hline & & $08-31-2000$ & 104.26 & & & 07-02-1959 & 74.77 & \\
\hline & & 09-05-2000 & 104.25 & & & 08-02-1959 & 75.69 & \\
\hline & & $09-10-2000$ & 104.69 & & & 09-09-1959 & 76.75 & \\
\hline & & $09-15-2000$ & 104.96 & & & $10-22-1959$ & 77.27 & \\
\hline & & $09-20-2000$ & 105.06 & & & $12-08-1959$ & 76.47 & \\
\hline
\end{tabular}


Table 2. Water levels in selected wells in Cedar Valley, Iron County, southwestern Utah-Continued

\begin{tabular}{|c|c|c|c|c|c|c|c|}
\hline Local well number & $\begin{array}{c}\text { Altitude of } \\
\text { land } \\
\text { surface } \\
\text { (feet) }\end{array}$ & Date & $\begin{array}{l}\text { Water level } \\
\text { (feet) }\end{array}$ & Local well number & $\begin{array}{l}\text { Altitude of } \\
\text { land } \\
\text { surface } \\
\text { (feet) }\end{array}$ & Date & $\begin{array}{l}\text { Water level } \\
\text { (feet) }\end{array}$ \\
\hline (C-36-11)8aca-1-Con & inued & 03-14-1960 & 74.93 & (C-36-11)8aca-1-Cc & inued & 09-10-1967 & 84.74 \\
\hline & & 05-03-1960 & 75.73 & & & 09-15-1967 & 85.02 \\
\hline & & 06-08-1960 & 77.74 & & & 09-20-1967 & 84.99 \\
\hline & & 07-07-1960 & 78.91 & & & 09-25-1967 & 84.50 \\
\hline & & 08-05-1960 & 79.64 & & & 09-30-1967 & 84.31 \\
\hline & & 09-08-1960 & 80.50 & & & $10-05-1967$ & 84.27 \\
\hline & & $10-31-1960$ & 80.60 & & & $10-10-1967$ & 84.03 \\
\hline & & $12-02-1960$ & 79.95 & & & $10-15-1967$ & 83.99 \\
\hline & & 04-04-1961 & 77.85 & & & $10-20-1967$ & 83.72 \\
\hline & & 05-09-1961 & 78.40 & & & $10-25-1967$ & 83.58 \\
\hline & & 05-25-1961 & 79.64 & & & $10-31-1967$ & 83.46 \\
\hline & & 10-16-1961 & 79.12 & & & 11-05-1967 & 83.31 \\
\hline & & 07-27-1966 & 86.08 & & & 11-10-1967 & 83.18 \\
\hline & & 09-27-1966 & 87.51 & & & $11-15-1967$ & 82.99 \\
\hline & & 03-06-1967 & 83.40 & & & $11-20-1967$ & 82.83 \\
\hline & & 03-10-1967 & 82.80 & & & $11-25-1967$ & 82.63 \\
\hline & & 03-15-1967 & 82.73 & & & 11-30-1967 & 82.39 \\
\hline & & 03-20-1967 & 82.57 & & & $12-05-1967$ & 82.28 \\
\hline & & 03-25-1967 & 82.62 & & & $12-10-1967$ & 82.14 \\
\hline & & 03-31-1967 & 82.49 & & & $12-15-1967$ & 81.85 \\
\hline & & 04-05-1967 & 82.46 & & & $12-20-1967$ & 81.75 \\
\hline & & 04-10-1967 & 82.20 & & & $12-25-1967$ & 81.64 \\
\hline & & 04-15-1967 & 82.13 & & & $12-31-1967$ & 81.40 \\
\hline & & 04-20-1967 & 82.27 & & & 01-05-1968 & 81.28 \\
\hline & & 04-25-1967 & 82.29 & & & 01-10-1968 & 81.08 \\
\hline & & 04-30-1967 & 82.68 & & & 01-15-1968 & 80.93 \\
\hline & & 05-05-1967 & 82.89 & & & 01-20-1968 & 80.86 \\
\hline & & 05-10-1967 & 83.17 & & & 01-25-1968 & 80.50 \\
\hline & & 05-15-1967 & 83.64 & & & 01-31-1968 & 80.37 \\
\hline & & 05-20-1967 & 83.40 & & & 02-05-1968 & 80.28 \\
\hline & & 05-25-1967 & 83.32 & & & 02-10-1968 & 80.03 \\
\hline & & 05-31-1967 & 82.98 & & & 02-15-1968 & 79.92 \\
\hline & & 06-05-1967 & 82.93 & & & 02-20-1968 & 79.72 \\
\hline & & 06-10-1967 & 83.24 & & & 02-25-1968 & 79.65 \\
\hline & & 06-15-1967 & 83.23 & & & $02-29-1968$ & 79.47 \\
\hline & & 06-20-1967 & 83.91 & & & 03-05-1968 & 79.27 \\
\hline & & 06-25-1967 & 82.92 & & & 03-10-1968 & 79.13 \\
\hline & & 06-30-1967 & 83.17 & & & 03-15-1968 & 79.04 \\
\hline & & 07-05-1967 & 83.28 & & & 03-20-1968 & 79.02 \\
\hline & & 07-10-1967 & 83.35 & & & 03-25-1968 & 78.70 \\
\hline & & 07-15-1967 & 83.57 & & & 03-31-1968 & 78.63 \\
\hline & & 07-20-1967 & 83.62 & & & 04-05-1968 & 78.53 \\
\hline & & 07-25-1967 & 83.92 & & & 04-10-1968 & 78.52 \\
\hline & & $07-31-1967$ & 84.23 & & & 04-15-1968 & 78.36 \\
\hline & & 08-05-1967 & 84.00 & & & 04-20-1968 & 78.34 \\
\hline & & 08-10-1967 & 83.82 & & & 04-25-1968 & 78.29 \\
\hline & & 08-15-1967 & 83.60 & & & 04-30-1968 & 78.60 \\
\hline & & 08-20-1967 & 84.02 & & & 05-05-1968 & 78.21 \\
\hline & & 08-25-1967 & 84.47 & & & 05-10-1968 & 78.09 \\
\hline & & 08-31-1967 & 84.44 & & & 05-15-1968 & 78.20 \\
\hline & & 09-05-1967 & 84.43 & & & $05-20-1968$ & 78.22 \\
\hline
\end{tabular}


Table 2. Water levels in selected wells in Cedar Valley, Iron County, southwestern Utah-Continued

\begin{tabular}{|c|c|c|c|c|c|c|c|}
\hline Local well number & $\begin{array}{c}\text { Altitude of } \\
\text { land } \\
\text { surface } \\
\text { (feet) }\end{array}$ & Date & $\begin{array}{c}\text { Water level } \\
\text { (feet) }\end{array}$ & Local well number & $\begin{array}{l}\text { Altitude of } \\
\text { land } \\
\text { surface } \\
\text { (feet) }\end{array}$ & Date & $\begin{array}{c}\text { Water level } \\
\text { (feet) }\end{array}$ \\
\hline \multirow{51}{*}{\multicolumn{2}{|c|}{ (C-36-11)8aca-1—Continued }} & $05-25-1968$ & 78.47 & \multirow{51}{*}{\multicolumn{2}{|c|}{ (C-36-11)8aca-1—Continued }} & $02-10-1969$ & 78.48 \\
\hline & & 05-31-1968 & 78.82 & & & $02-15-1969$ & 78.31 \\
\hline & & 06-05-1968 & 78.94 & & & 02-20-1969 & 78.25 \\
\hline & & 06-10-1968 & 79.03 & & & 02-25-1969 & 78.07 \\
\hline & & 06-15-1968 & 79.20 & & & 02-28-1969 & 77.98 \\
\hline & & 06-20-1968 & 79.42 & & & 03-05-1969 & 77.88 \\
\hline & & $06-25-1968$ & 79.54 & & & 03-10-1969 & 77.83 \\
\hline & & 06-30-1968 & 79.77 & & & 03-15-1969 & 77.81 \\
\hline & & 07-05-1968 & 80.06 & & & 03-20-1969 & 77.55 \\
\hline & & 07-10-1968 & 80.33 & & & 03-25-1969 & 77.55 \\
\hline & & 07-15-1968 & 80.54 & & & 03-31-1969 & 77.30 \\
\hline & & 07-20-1968 & 80.82 & & & 04-05-1969 & 77.16 \\
\hline & & 07-25-1968 & 80.98 & & & 04-10-1969 & 77.20 \\
\hline & & 07-31-1968 & 80.86 & & & 04-15-1969 & 77.10 \\
\hline & & 08-05-1968 & 80.61 & & & 04-20-1969 & 77.07 \\
\hline & & 08-10-1968 & 80.66 & & & 04-25-1969 & 77.01 \\
\hline & & 08-15-1968 & 80.87 & & & 04-30-1969 & 76.90 \\
\hline & & 08-20-1968 & 80.75 & & & 05-05-1969 & 76.95 \\
\hline & & 08-25-1968 & 81.10 & & & 05-10-1969 & 76.85 \\
\hline & & 08-31-1968 & 81.42 & & & 05-15-1969 & 76.77 \\
\hline & & 09-05-1968 & 81.65 & & & 05-20-1969 & 76.70 \\
\hline & & 09-10-1968 & 81.75 & & & 05-25-1969 & 76.60 \\
\hline & & 09-15-1968 & 81.86 & & & 05-31-1969 & 76.52 \\
\hline & & 09-20-1968 & 81.87 & & & 06-05-1969 & 76.83 \\
\hline & & 09-25-1968 & 81.88 & & & 06-10-1969 & 76.31 \\
\hline & & 09-30-1968 & 81.88 & & & 06-15-1969 & 76.00 \\
\hline & & $10-05-1968$ & 81.89 & & & 06-20-1969 & 76.22 \\
\hline & & $10-10-1968$ & 81.97 & & & 06-25-1969 & 76.10 \\
\hline & & $10-15-1968$ & 81.96 & & & 06-30-1969 & 76.21 \\
\hline & & $10-20-1968$ & 81.82 & & & 07-05-1969 & 76.15 \\
\hline & & $10-25-1968$ & 81.85 & & & 07-10-1969 & 76.15 \\
\hline & & $10-31-1968$ & 81.50 & & & 07-15-1969 & 76.86 \\
\hline & & $11-05-1968$ & 81.32 & & & 07-20-1969 & 77.00 \\
\hline & & $11-10-1968$ & 81.28 & & & $07-25-1969$ & 76.71 \\
\hline & & $11-15-1968$ & 80.96 & & & 07-31-1969 & 76.83 \\
\hline & & $11-20-1968$ & 80.90 & & & 08-05-1969 & 76.70 \\
\hline & & $11-25-1968$ & 80.64 & & & 08-10-1969 & 76.98 \\
\hline & & $11-30-1968$ & 80.41 & & & 08-15-1969 & 77.35 \\
\hline & & $12-05-1968$ & 80.30 & & & 08-20-1969 & 77.60 \\
\hline & & $12-10-1968$ & 80.08 & & & 08-25-1969 & 77.70 \\
\hline & & $12-15-1968$ & 79.96 & & & 08-31-1969 & 78.10 \\
\hline & & $12-20-1968$ & 79.75 & & & 09-05-1969 & 78.22 \\
\hline & & $12-25-1968$ & 79.58 & & & 09-10-1969 & 78.41 \\
\hline & & $12-31-1968$ & 79.58 & & & 09-15-1969 & 78.31 \\
\hline & & 01-05-1969 & 79.37 & & & 09-20-1969 & 78.21 \\
\hline & & 01-10-1969 & 79.24 & & & 09-25-1969 & 78.36 \\
\hline & & 01-15-1969 & 79.12 & & & 09-30-1969 & 78.33 \\
\hline & & 01-20-1969 & 78.98 & & & $10-05-1969$ & 78.46 \\
\hline & & 01-25-1969 & 78.79 & & & $10-10-1969$ & 78.10 \\
\hline & & 01-31-1969 & 78.72 & & & $10-15-1969$ & 78.09 \\
\hline & & 02-05-1969 & 78.50 & & & $10-20-1969$ & 78.01 \\
\hline
\end{tabular}


Table 2. Water levels in selected wells in Cedar Valley, Iron County, southwestern Utah-Continued

\begin{tabular}{|c|c|c|c|c|c|c|c|}
\hline Local well number & $\begin{array}{c}\text { Altitude of } \\
\text { land } \\
\text { surface } \\
\text { (feet) }\end{array}$ & Date & $\begin{array}{c}\text { Water level } \\
\text { (feet) }\end{array}$ & Local well number & $\begin{array}{c}\text { Altitude of } \\
\text { land } \\
\text { surface } \\
\text { (feet) }\end{array}$ & Date & $\begin{array}{c}\text { Water level } \\
\text { (feet) }\end{array}$ \\
\hline (C-36-11)8aca-1-Con & nued & $10-25-1969$ & 77.91 & $(\mathrm{C}-36-11) 8 \mathrm{aca}-1-\mathrm{Cc}$ & inued & $07-10-1970$ & 78.86 \\
\hline & & $10-31-1969$ & 77.76 & & & $07-15-1970$ & 79.10 \\
\hline & & 11-05-1969 & 77.55 & & & $07-20-1970$ & 79.37 \\
\hline & & 11-10-1969 & 77.42 & & & $07-25-1970$ & 79.18 \\
\hline & & $11-15-1969$ & 77.17 & & & 07-31-1970 & 79.43 \\
\hline & & $11-20-1969$ & 77.14 & & & 08-05-1970 & 79.54 \\
\hline & & $11-25-1969$ & 76.99 & & & 08-10-1970 & 79.72 \\
\hline & & $11-30-1969$ & 76.82 & & & $08-15-1970$ & 80.08 \\
\hline & & 12-05-1969 & 76.66 & & & 08-20-1970 & 80.38 \\
\hline & & $12-10-1969$ & 76.54 & & & $08-25-1970$ & 80.64 \\
\hline & & $12-15-1969$ & 76.39 & & & 08-31-1970 & 80.88 \\
\hline & & $12-20-1969$ & 76.25 & & & 09-05-1970 & 81.08 \\
\hline & & $12-25-1969$ & 76.06 & & & 09-10-1970 & 81.35 \\
\hline & & $12-31-1969$ & 75.95 & & & 09-15-1970 & 81.33 \\
\hline & & 01-05-1970 & 75.92 & & & 09-20-1970 & 81.34 \\
\hline & & 01-10-1970 & 75.67 & & & $09-25-1970$ & 81.58 \\
\hline & & 01-15-1970 & 75.55 & & & 09-30-1970 & 81.73 \\
\hline & & 01-20-1970 & 75.54 & & & $10-05-1970$ & 81.65 \\
\hline & & $01-25-1970$ & 75.31 & & & $10-10-1970$ & 81.71 \\
\hline & & 01-31-1970 & 75.13 & & & $10-15-1970$ & 81.76 \\
\hline & & 02-05-1970 & 75.06 & & & $10-20-1970$ & 81.68 \\
\hline & & 02-10-1970 & 74.93 & & & $10-25-1970$ & 81.72 \\
\hline & & 02-15-1970 & 74.88 & & & $10-31-1970$ & 81.75 \\
\hline & & $02-20-1970$ & 74.68 & & & $11-05-1970$ & 81.53 \\
\hline & & $02-25-1970$ & 74.58 & & & $11-10-1970$ & 81.38 \\
\hline & & 02-28-1970 & 74.37 & & & $11-15-1970$ & 81.32 \\
\hline & & 03-05-1970 & 74.30 & & & $11-20-1970$ & 81.26 \\
\hline & & $03-10-1970$ & 74.10 & & & $11-25-1970$ & 81.10 \\
\hline & & 03-15-1970 & 74.09 & & & $11-30-1970$ & 80.99 \\
\hline & & 03-20-1970 & 74.00 & & & $12-05-1970$ & 80.94 \\
\hline & & $03-25-1970$ & 74.09 & & & $12-10-1970$ & 80.78 \\
\hline & & 03-31-1970 & 73.95 & & & $12-15-1970$ & 80.57 \\
\hline & & 04-05-1970 & 73.89 & & & $12-20-1970$ & 80.37 \\
\hline & & 04-10-1970 & 73.93 & & & $12-25-1970$ & 80.30 \\
\hline & & 04-15-1970 & 74.10 & & & $12-31-1970$ & 80.40 \\
\hline & & 04-20-1970 & 74.31 & & & 01-05-1971 & 80.02 \\
\hline & & 04-25-1970 & 74.87 & & & 01-10-1971 & 79.83 \\
\hline & & 04-30-1970 & 75.17 & & & 01-15-1971 & 79.81 \\
\hline & & 05-05-1970 & 75.55 & & & 01-20-1971 & 79.58 \\
\hline & & 05-10-1970 & 75.65 & & & 01-25-1971 & 79.53 \\
\hline & & 05-15-1970 & 76.20 & & & 01-31-1971 & 79.28 \\
\hline & & $05-20-1970$ & 76.34 & & & 02-05-1971 & 79.14 \\
\hline & & $05-25-1970$ & 76.73 & & & 02-10-1971 & 79.07 \\
\hline & & 05-31-1970 & 77.15 & & & 02-15-1971 & 78.87 \\
\hline & & 06-05-1970 & 77.42 & & & 02-20-1971 & 78.62 \\
\hline & & 06-10-1970 & 77.11 & & & 02-25-1971 & 78.43 \\
\hline & & $06-15-1970$ & 77.40 & & & 02-28-1971 & 78.43 \\
\hline & & 06-20-1970 & 77.70 & & & 03-05-1971 & 78.35 \\
\hline & & $06-25-1970$ & 78.06 & & & 03-10-1971 & 78.23 \\
\hline & & 06-30-1970 & 78.25 & & & 03-15-1971 & 78.18 \\
\hline & & 07-05-1970 & 78.58 & & & 03-20-1971 & 78.02 \\
\hline
\end{tabular}


Table 2. Water levels in selected wells in Cedar Valley, Iron County, southwestern Utah-Continued

\begin{tabular}{|c|c|c|c|c|c|}
\hline $\begin{array}{l}\text { Altitude of } \\
\text { land } \\
\text { surface } \\
\text { (feet) }\end{array}$ & Date & $\begin{array}{c}\text { Water level } \\
\text { (feet) }\end{array}$ & $\begin{array}{l}\text { Altitude of } \\
\text { land } \\
\text { surface } \\
\text { (feet) }\end{array}$ & Date & $\begin{array}{l}\text { Water level } \\
\text { (feet) }\end{array}$ \\
\hline \multirow[t]{51}{*}{ (C-36-11)8aca-1-Continued } & $03-25-1971$ & 77.87 & (C-36-11)8aca-1-Continued & $12-10-1971$ & 82.21 \\
\hline & 03-31-1971 & 77.68 & & $12-15-1971$ & 82.06 \\
\hline & 04-05-1971 & 77.68 & & $12-20-1971$ & 81.92 \\
\hline & 04-10-1971 & 77.78 & & $12-25-1971$ & 81.66 \\
\hline & 04-15-1971 & 77.92 & & $12-31-1971$ & 81.57 \\
\hline & 04-20-1971 & 77.91 & & 01-05-1972 & 81.44 \\
\hline & $04-25-1971$ & 77.92 & & 01-10-1972 & 81.26 \\
\hline & 04-30-1971 & 78.56 & & 01-15-1972 & 81.12 \\
\hline & 05-05-1971 & 78.72 & & 01-20-1972 & 80.93 \\
\hline & 05-10-1971 & 78.96 & & 01-25-1972 & 80.74 \\
\hline & 05-15-1971 & 79.15 & & 01-31-1972 & 80.48 \\
\hline & 05-20-1971 & 79.23 & & 02-05-1972 & 80.39 \\
\hline & 05-25-1971 & 79.35 & & 02-10-1972 & 80.29 \\
\hline & 05-31-1971 & 79.62 & & 02-15-1972 & 80.12 \\
\hline & 06-05-1971 & 80.05 & & 02-20-1972 & 79.97 \\
\hline & 06-10-1971 & 80.33 & & 02-25-1972 & 79.74 \\
\hline & 06-15-1971 & 80.51 & & 02-29-1972 & 79.57 \\
\hline & 06-20-1971 & 80.64 & & 03-05-1972 & 79.52 \\
\hline & 06-25-1971 & 80.93 & & 03-10-1972 & 79.34 \\
\hline & 06-30-1971 & 81.18 & & 03-15-1972 & 79.22 \\
\hline & 07-05-1971 & 81.31 & & 03-20-1972 & 79.13 \\
\hline & 07-10-1971 & 81.69 & & 03-25-1972 & 78.99 \\
\hline & 07-15-1971 & 81.91 & & 03-31-1972 & 79.06 \\
\hline & $07-20-1971$ & 82.14 & & 04-05-1972 & 78.99 \\
\hline & 07-25-1971 & 82.30 & & 04-10-1972 & 79.25 \\
\hline & 07-31-1971 & 82.54 & & 04-15-1972 & 79.46 \\
\hline & 08-05-1971 & 82.63 & & 04-20-1972 & 79.54 \\
\hline & 08-10-1971 & 82.79 & & 04-25-1972 & 79.57 \\
\hline & 08-15-1971 & 82.98 & & 04-30-1972 & 80.23 \\
\hline & 08-20-1971 & 83.25 & & 05-05-1972 & 80.35 \\
\hline & $08-25-1971$ & 83.49 & & 05-10-1972 & 80.58 \\
\hline & 08-31-1971 & 83.64 & & 05-15-1972 & 80.87 \\
\hline & 09-05-1971 & 83.79 & & 05-20-1972 & 81.12 \\
\hline & 09-10-1971 & 83.88 & & 05-25-1972 & 81.35 \\
\hline & 09-15-1971 & 83.83 & & 05-31-1972 & 81.65 \\
\hline & 09-20-1971 & 84.11 & & 06-05-1972 & 81.89 \\
\hline & 09-25-1971 & 84.13 & & 06-10-1972 & 82.06 \\
\hline & 09-30-1971 & 84.27 & & 06-15-1972 & 82.35 \\
\hline & $10-05-1971$ & 84.24 & & 06-20-1972 & 82.61 \\
\hline & $10-10-1971$ & 84.25 & & 06-25-1972 & 82.83 \\
\hline & $10-15-1971$ & 84.40 & & 06-30-1972 & 83.11 \\
\hline & $10-20-1971$ & 84.14 & & 07-05-1972 & 83.42 \\
\hline & $10-25-1971$ & 84.00 & & 07-10-1972 & 83.70 \\
\hline & $10-31-1971$ & 83.76 & & 07-15-1972 & 84.06 \\
\hline & 11-05-1971 & 83.59 & & 07-20-1972 & 84.12 \\
\hline & 11-10-1971 & 83.43 & & 07-25-1972 & 84.35 \\
\hline & $11-15-1971$ & 83.17 & & 07-31-1972 & 84.80 \\
\hline & $11-20-1971$ & 82.99 & & 08-05-1972 & 85.11 \\
\hline & $11-25-1971$ & 82.84 & & 08-10-1972 & 85.22 \\
\hline & 11-30-1971 & 82.63 & & 08-15-1972 & 85.59 \\
\hline & 12-05-1971 & 82.45 & & 08-20-1972 & 85.84 \\
\hline
\end{tabular}


Table 2. Water levels in selected wells in Cedar Valley, Iron County, southwestern Utah-Continued

\begin{tabular}{|c|c|c|c|c|c|c|c|}
\hline Local well number & $\begin{array}{c}\text { Altitude of } \\
\text { land } \\
\text { surface } \\
\text { (feet) }\end{array}$ & Date & $\begin{array}{c}\text { Water level } \\
\text { (feet) }\end{array}$ & Local well number & $\begin{array}{l}\text { Altitude of } \\
\text { land } \\
\text { surface } \\
\text { (feet) }\end{array}$ & Date & $\begin{array}{c}\text { Water level } \\
\text { (feet) }\end{array}$ \\
\hline \multirow{51}{*}{\multicolumn{2}{|c|}{ (C-36-11)8aca-1-Continued }} & $08-25-1972$ & 86.44 & \multirow{51}{*}{\multicolumn{2}{|c|}{ (C-36-11)8aca-1-Continued }} & $05-10-1973$ & 78.01 \\
\hline & & 08-31-1972 & 86.34 & & & $05-15-1973$ & 77.91 \\
\hline & & 09-05-1972 & 86.27 & & & 05-20-1973 & 77.61 \\
\hline & & 09-10-1972 & 86.37 & & & $05-25-1973$ & 77.36 \\
\hline & & 09-15-1972 & 86.34 & & & 05-31-1973 & 77.30 \\
\hline & & 09-20-1972 & 86.27 & & & 06-05-1973 & 76.95 \\
\hline & & 09-25-1972 & 85.96 & & & 06-10-1973 & 76.49 \\
\hline & & 09-30-1972 & 86.07 & & & 06-15-1973 & 76.13 \\
\hline & & $10-05-1972$ & 85.89 & & & 06-20-1973 & 75.65 \\
\hline & & 10-10-1972 & 85.68 & & & $06-25-1973$ & 75.52 \\
\hline & & $10-15-1972$ & 85.50 & & & 06-30-1973 & 76.55 \\
\hline & & $10-20-1972$ & 85.36 & & & 07-05-1973 & 76.32 \\
\hline & & $10-25-1972$ & 85.17 & & & $07-10-1973$ & 75.86 \\
\hline & & $10-31-1972$ & 85.04 & & & $07-15-1973$ & 76.78 \\
\hline & & 11-05-1972 & 84.84 & & & 07-20-1973 & 75.97 \\
\hline & & $11-10-1972$ & 84.63 & & & $07-25-1973$ & 76.30 \\
\hline & & 11-15-1972 & 84.51 & & & $07-31-1973$ & 77.20 \\
\hline & & 11-20-1972 & 84.31 & & & 08-05-1973 & 76.73 \\
\hline & & $11-25-1972$ & 84.28 & & & 08-10-1973 & 76.38 \\
\hline & & 11-30-1972 & 84.12 & & & 08-15-1973 & 77.26 \\
\hline & & $12-05-1972$ & 83.89 & & & 08-20-1973 & 77.49 \\
\hline & & $12-10-1972$ & 83.70 & & & $08-25-1973$ & 77.32 \\
\hline & & $12-15-1972$ & 83.59 & & & 08-31-1973 & 77.78 \\
\hline & & $12-20-1972$ & 83.38 & & & 09-05-1973 & 77.75 \\
\hline & & $12-25-1972$ & 83.25 & & & 09-10-1973 & 77.75 \\
\hline & & $12-31-1972$ & 82.92 & & & $09-15-1973$ & 77.53 \\
\hline & & 01-05-1973 & 82.77 & & & 09-20-1973 & 77.33 \\
\hline & & 01-10-1973 & 82.61 & & & 09-25-1973 & 77.29 \\
\hline & & 01-15-1973 & 82.31 & & & 09-30-1973 & 77.32 \\
\hline & & 01-20-1973 & 82.12 & & & $10-05-1973$ & 77.25 \\
\hline & & 01-25-1973 & 81.82 & & & $10-10-1973$ & 77.22 \\
\hline & & 01-31-1973 & 81.55 & & & $10-15-1973$ & 77.23 \\
\hline & & 02-05-1973 & 81.34 & & & $10-20-1973$ & 77.05 \\
\hline & & 02-10-1973 & 81.02 & & & $10-25-1973$ & 76.78 \\
\hline & & 02-15-1973 & 80.93 & & & $10-30-1973$ & 76.70 \\
\hline & & 02-20-1973 & 80.72 & & & 11-05-1973 & 76.50 \\
\hline & & 02-25-1973 & 80.52 & & & 11-10-1973 & 76.40 \\
\hline & & 02-28-1973 & 80.33 & & & $11-15-1973$ & 76.20 \\
\hline & & 03-05-1973 & 80.16 & & & $11-20-1973$ & 76.10 \\
\hline & & 03-10-1973 & 79.95 & & & $11-25-1973$ & 76.00 \\
\hline & & 03-15-1973 & 79.91 & & & $11-30-1973$ & 75.80 \\
\hline & & 03-20-1973 & 79.53 & & & $12-05-1973$ & 75.70 \\
\hline & & 03-25-1973 & 79.42 & & & $12-10-1973$ & 75.50 \\
\hline & & 03-31-1973 & 79.21 & & & $12-15-1973$ & 75.30 \\
\hline & & 04-05-1973 & 78.99 & & & $12-20-1973$ & 75.32 \\
\hline & & 04-10-1973 & 78.87 & & & $12-25-1973$ & 75.10 \\
\hline & & 04-15-1973 & 78.69 & & & $12-30-1973$ & 74.90 \\
\hline & & 04-20-1973 & 78.53 & & & 01-05-1974 & 74.68 \\
\hline & & 04-25-1973 & 78.46 & & & 01-10-1974 & 74.72 \\
\hline & & 04-30-1973 & 78.20 & & & 01-15-1974 & 74.58 \\
\hline & & 05-05-1973 & 78.05 & & & 01-20-1974 & 74.34 \\
\hline
\end{tabular}


Table 2. Water levels in selected wells in Cedar Valley, Iron County, southwestern Utah-Continued

\begin{tabular}{|c|c|c|c|c|c|}
\hline $\begin{array}{c}\text { Altitude of } \\
\text { land } \\
\text { surface } \\
\text { (feet) }\end{array}$ & Date & $\begin{array}{c}\text { Water level } \\
\text { (feet) }\end{array}$ & $\begin{array}{l}\text { Altitude of } \\
\text { land } \\
\text { surface } \\
\text { (feet) }\end{array}$ & Date & $\begin{array}{l}\text { Water level } \\
\text { (feet) }\end{array}$ \\
\hline \multirow[t]{51}{*}{ (C-36-11)8aca-1—Continued } & $01-25-1974$ & 74.24 & (C-36-11)8aca-1-Continued & $10-10-1974$ & 81.14 \\
\hline & 01-31-1974 & 74.12 & & $10-15-1974$ & 81.19 \\
\hline & 02-05-1974 & 73.99 & & $10-20-1974$ & 81.16 \\
\hline & 02-10-1974 & 73.94 & & $10-25-1974$ & 81.19 \\
\hline & 02-15-1974 & 73.77 & & $10-31-1974$ & 81.12 \\
\hline & 02-20-1974 & 73.65 & & 11-05-1974 & 80.96 \\
\hline & 02-25-1974 & 73.51 & & $11-10-1974$ & 80.81 \\
\hline & 02-28-1974 & 73.44 & & $11-15-1974$ & 80.69 \\
\hline & 03-05-1974 & 73.22 & & $11-20-1974$ & 80.45 \\
\hline & 03-10-1974 & 73.23 & & $11-25-1974$ & 80.40 \\
\hline & 03-15-1974 & 73.08 & & $11-30-1974$ & 80.16 \\
\hline & 03-20-1974 & 72.94 & & $12-05-1974$ & 80.06 \\
\hline & 03-25-1974 & 72.85 & & $12-10-1974$ & 79.96 \\
\hline & 03-31-1974 & 72.69 & & $12-15-1974$ & 79.81 \\
\hline & 04-05-1974 & 72.64 & & $12-20-1974$ & 79.20 \\
\hline & $04-10-1974$ & 72.65 & & $12-25-1974$ & 79.50 \\
\hline & 04-15-1974 & 72.83 & & $12-31-1974$ & 79.58 \\
\hline & 04-20-1974 & 72.62 & & 01-05-1975 & 79.31 \\
\hline & 04-25-1974 & 72.80 & & 01-10-1975 & 79.08 \\
\hline & 04-30-1974 & 73.58 & & 01-15-1975 & 79.04 \\
\hline & 05-05-1974 & 73.40 & & $01-20-1975$ & 78.90 \\
\hline & 05-10-1974 & 73.88 & & 01-25-1975 & 78.70 \\
\hline & 05-15-1974 & 74.67 & & 01-31-1975 & 78.61 \\
\hline & $05-20-1974$ & 74.74 & & 02-05-1975 & 78.50 \\
\hline & 05-25-1974 & 75.98 & & 02-10-1975 & 78.24 \\
\hline & 05-31-1974 & 76.81 & & 02-15-1975 & 78.12 \\
\hline & 06-05-1974 & 76.65 & & 02-20-1975 & 77.98 \\
\hline & 06-10-1974 & 76.80 & & 02-25-1975 & 77.93 \\
\hline & $06-15-1974$ & 76.65 & & $02-28-1975$ & 77.87 \\
\hline & $06-20-1974$ & 76.85 & & 03-05-1975 & 77.63 \\
\hline & 06-25-1974 & 77.08 & & 03-10-1975 & 77.45 \\
\hline & 06-30-1974 & 78.00 & & 03-15-1975 & 77.18 \\
\hline & 07-05-1974 & 78.52 & & 03-20-1975 & 77.01 \\
\hline & 07-10-1974 & 78.97 & & 03-25-1975 & 76.75 \\
\hline & 07-15-1974 & 78.98 & & 03-31-1975 & 76.68 \\
\hline & $07-20-1974$ & 78.99 & & 04-05-1975 & 76.60 \\
\hline & $07-25-1974$ & 79.81 & & 04-10-1975 & 76.53 \\
\hline & 07-31-1974 & 79.63 & & 04-15-1975 & 76.42 \\
\hline & 08-05-1974 & 79.52 & & 04-20-1975 & 76.42 \\
\hline & 08-10-1974 & 79.46 & & 04-25-1975 & 76.23 \\
\hline & 08-15-1974 & 80.45 & & 04-30-1975 & 76.30 \\
\hline & 08-20-1974 & 80.82 & & 05-05-1975 & 76.14 \\
\hline & 08-25-1974 & 80.75 & & 05-10-1975 & 76.38 \\
\hline & 08-31-1974 & 81.38 & & 05-15-1975 & 77.70 \\
\hline & 09-05-1974 & 81.68 & & 05-20-1975 & 77.12 \\
\hline & 09-10-1974 & 81.24 & & 05-25-1975 & 77.71 \\
\hline & 09-15-1974 & 81.26 & & 05-31-1975 & 77.76 \\
\hline & 09-20-1974 & 81.00 & & 06-05-1975 & 78.05 \\
\hline & 09-25-1974 & 81.12 & & 06-10-1975 & 78.11 \\
\hline & 09-30-1974 & 81.08 & & 06-15-1975 & 78.01 \\
\hline & $10-05-1974$ & 80.98 & & $06-20-1975$ & 78.00 \\
\hline
\end{tabular}


Table 2. Water levels in selected wells in Cedar Valley, Iron County, southwestern Utah-Continued

\begin{tabular}{|c|c|c|c|c|c|c|}
\hline Local well number & $\begin{array}{c}\text { Altitude of } \\
\text { land } \\
\text { surface } \\
\text { (feet) }\end{array}$ & Date & $\begin{array}{l}\text { Water level } \\
\text { (feet) }\end{array}$ & $\begin{array}{l}\text { Altitude of } \\
\text { land } \\
\text { surface } \\
\text { (feet) }\end{array}$ & Date & $\begin{array}{c}\text { Water level } \\
\text { (feet) }\end{array}$ \\
\hline \multirow{51}{*}{\multicolumn{2}{|c|}{ (C-36-11)8aca-1-Continued }} & $06-25-1975$ & 78.30 & (C-36-11)8aca-1—Continued & 03-10-1976 & 77.97 \\
\hline & & 06-30-1975 & 78.80 & & 03-15-1976 & 77.94 \\
\hline & & 07-05-1975 & 79.50 & & 03-20-1976 & 77.80 \\
\hline & & 07-10-1975 & 79.59 & & 03-25-1976 & 77.47 \\
\hline & & 07-15-1975 & 79.17 & & 03-31-1976 & 77.39 \\
\hline & & 07-20-1975 & 79.59 & & 04-05-1976 & 77.41 \\
\hline & & $07-25-1975$ & 80.48 & & 04-10-1976 & 77.44 \\
\hline & & 07-31-1975 & 80.49 & & 04-15-1976 & 77.16 \\
\hline & & 08-05-1975 & 80.98 & & 04-20-1976 & 77.28 \\
\hline & & 08-10-1975 & 81.08 & & $04-25-1976$ & 77.27 \\
\hline & & 08-15-1975 & 81.64 & & 04-30-1976 & 77.58 \\
\hline & & 08-20-1975 & 81.17 & & 05-05-1976 & 78.78 \\
\hline & & 08-25-1975 & 81.19 & & 05-10-1976 & 76.41 \\
\hline & & 08-31-1975 & 82.08 & & $05-15-1976$ & 79.09 \\
\hline & & 09-05-1975 & 82.55 & & 05-20-1976 & 79.05 \\
\hline & & 09-10-1975 & 82.35 & & 05-25-1976 & 80.30 \\
\hline & & 09-15-1975 & 82.30 & & 05-31-1976 & 80.77 \\
\hline & & 09-20-1975 & 82.25 & & 06-05-1976 & 81.08 \\
\hline & & 09-25-1975 & 82.20 & & 06-10-1976 & 81.05 \\
\hline & & 09-30-1975 & 82.15 & & 06-15-1976 & 81.45 \\
\hline & & $10-05-1975$ & 82.10 & & 06-20-1976 & 81.36 \\
\hline & & $10-10-1975$ & 82.09 & & $06-25-1976$ & 82.08 \\
\hline & & $10-15-1975$ & 82.09 & & 06-30-1976 & 82.72 \\
\hline & & $10-20-1975$ & 82.09 & & 07-05-1976 & 83.02 \\
\hline & & $10-25-1975$ & 82.09 & & 07-10-1976 & 83.47 \\
\hline & & $10-31-1975$ & 82.09 & & 07-15-1976 & 83.49 \\
\hline & & $11-05-1975$ & 82.09 & & 07-20-1976 & 84.08 \\
\hline & & $11-10-1975$ & 82.09 & & 07-25-1976 & 83.87 \\
\hline & & $11-15-1975$ & 82.09 & & 07-31-1976 & 83.80 \\
\hline & & $11-20-1975$ & 82.09 & & 08-05-1976 & 84.02 \\
\hline & & $11-25-1975$ & 82.09 & & 08-10-1976 & 84.80 \\
\hline & & $11-30-1975$ & 82.09 & & 08-15-1976 & 85.12 \\
\hline & & $12-05-1975$ & 82.09 & & 08-20-1976 & 85.54 \\
\hline & & $12-10-1975$ & 80.50 & & 08-25-1976 & 85.88 \\
\hline & & $12-15-1975$ & 80.43 & & 08-31-1976 & 85.85 \\
\hline & & $12-20-1975$ & 80.24 & & 09-05-1976 & 86.54 \\
\hline & & $12-25-1975$ & 80.11 & & 09-10-1976 & 86.95 \\
\hline & & $12-31-1975$ & 79.80 & & 09-15-1976 & 87.19 \\
\hline & & 01-05-1976 & 79.71 & & 09-20-1976 & 86.85 \\
\hline & & 01-10-1976 & 79.64 & & 09-25-1976 & 86.73 \\
\hline & & 01-15-1976 & 79.60 & & 09-30-1976 & 86.83 \\
\hline & & 01-20-1976 & 79.48 & & $10-05-1976$ & 86.81 \\
\hline & & 01-25-1976 & 79.28 & & $10-10-1976$ & 86.50 \\
\hline & & 01-31-1976 & 79.16 & & $10-15-1976$ & 86.39 \\
\hline & & 02-05-1976 & 78.95 & & $10-20-1976$ & 86.33 \\
\hline & & 02-10-1976 & 78.86 & & $10-25-1976$ & 86.12 \\
\hline & & 02-15-1976 & 78.61 & & $10-31-1976$ & 86.10 \\
\hline & & 02-20-1976 & 78.67 & & $11-05-1976$ & 85.98 \\
\hline & & $02-25-1976$ & 78.43 & & $11-10-1976$ & 85.82 \\
\hline & & 02-29-1976 & 78.16 & & $11-15-1976$ & 85.73 \\
\hline & & 03-05-1976 & 78.25 & & $11-20-1976$ & 85.51 \\
\hline
\end{tabular}


Table 2. Water levels in selected wells in Cedar Valley, Iron County, southwestern Utah-Continued

\begin{tabular}{|c|c|c|c|c|c|c|c|}
\hline $\begin{array}{l}\text { Altitude of } \\
\text { land } \\
\text { surface } \\
\text { (feet) }\end{array}$ & Date & $\begin{array}{c}\text { Water level } \\
\text { (feet) }\end{array}$ & Local well number & $\begin{array}{c}\text { Altitude of } \\
\text { land } \\
\text { surface } \\
\text { (feet) }\end{array}$ & Date & \multicolumn{2}{|c|}{$\begin{array}{l}\text { Water level } \\
\text { (feet) }\end{array}$} \\
\hline \multirow[t]{51}{*}{ (C-36-11)8aca-1-Continued } & $11-25-1976$ & 85.26 & \multicolumn{2}{|c|}{ (C-36-11)8aca-1-Continued } & 08-10-1977 & 89.43 & \\
\hline & $11-30-1976$ & 85.20 & & & 08-15-1977 & 90.28 & \\
\hline & $12-05-1976$ & 84.99 & & & 08-20-1977 & 90.09 & \\
\hline & $12-10-1976$ & 84.87 & & & $08-25-1977$ & 90.44 & \\
\hline & $12-15-1976$ & 84.67 & & & 08-31-1977 & 90.97 & \\
\hline & $12-20-1976$ & 84.46 & & & 09-05-1977 & 91.30 & \\
\hline & $12-25-1976$ & 84.37 & & & 09-10-1977 & 91.28 & \\
\hline & $12-31-1976$ & 84.08 & & & 03-05-1979 & 78.66 & \\
\hline & 01-05-1977 & 83.92 & & & $10-02-1979$ & 89.77 & \\
\hline & 01-10-1977 & 83.82 & & & & & \\
\hline & 01-15-1977 & 83.70 & (C-36-11)8bbb-1 & 5,537 & 07-16-1998 & 92.90 & \\
\hline & 01-20-1977 & 83.53 & & & $11-19-1998$ & 63.78 & \\
\hline & 01-25-1977 & 83.41 & & & 03-16-1999 & 55.25 & \\
\hline & 01-31-1977 & 83.19 & & & 11-02-1999 & 72.33 & \\
\hline & 02-05-1977 & 83.10 & & & $03-14-2000$ & 59.60 & \\
\hline & 02-10-1977 & 83.02 & & & & & \\
\hline & 02-15-1977 & 82.83 & (C-36-11)8cbb-1 & 5,539 & $11-30-1955$ & 47 & $\mathrm{D}$ \\
\hline & 02-20-1977 & 82.66 & & & $11-18-1974$ & 46 & $\mathrm{R}$ \\
\hline & 02-25-1977 & 82.58 & & & $11-18-1998$ & 69.05 & \\
\hline & 02-28-1977 & 82.40 & & & 03-16-1999 & 61.15 & \\
\hline & 03-05-1977 & 82.40 & & & 11-02-1999 & 74.66 & \\
\hline & 03-10-1977 & 82.08 & & & $03-14-2000$ & 64.20 & \\
\hline & 03-15-1977 & 82.06 & & & & & \\
\hline & 03-20-1977 & 81.97 & (C-36-11)8dab-1 & 5,590 & 05-15-1998 & 95.10 & \\
\hline & 03-25-1977 & 82.02 & & & 11-19-1998 & 101.70 & \\
\hline & 03-31-1977 & 82.08 & & & 03-16-1999 & 93.92 & \\
\hline & 04-05-1977 & 82.29 & & & $12-02-1999$ & 105.50 & \\
\hline & 04-10-1977 & 82.42 & & & 03-14-2000 & 97.07 & \\
\hline & 04-15-1977 & 83.75 & & & & & \\
\hline & 04-20-1977 & 84.10 & (C-36-11)11bac-1 & 5,758 & $02-02-2000$ & 246.69 & \\
\hline & 04-25-1977 & 84.20 & & & 03-14-2000 & 245.54 & \\
\hline & 04-30-1977 & 84.30 & & & 03-08-2001 & 249.72 & \\
\hline & 05-05-1977 & 84.40 & & & & & \\
\hline & 05-10-1977 & 84.45 & (C-36-11)13adb-1 & 6,020 & 03-16-1998 & 30.95 & \\
\hline & 05-15-1977 & 84.50 & & & $11-19-1998$ & 30.52 & \\
\hline & 05-20-1977 & 85.04 & & & 03-15-1999 & 30.15 & \\
\hline & 05-25-1977 & 85.56 & & & 11-02-1999 & 35.34 & \\
\hline & 05-31-1977 & 86.08 & & & 03-14-2000 & 33.04 & \\
\hline & 06-05-1977 & 85.97 & & & 03-09-2001 & 32.10 & \\
\hline & 06-10-1977 & 86.12 & & & & & \\
\hline & 06-15-1977 & 86.52 & (C-36-11)18aaa-1 & 5,530 & $10-17-1985$ & 71 & $\mathrm{D}$ \\
\hline & 06-20-1977 & 86.54 & & & 03-16-1998 & 73.44 & \\
\hline & 06-25-1977 & 86.65 & & & $11-18-1998$ & 81.28 & \\
\hline & 06-30-1977 & 87.38 & & & 03-16-1999 & 73.09 & \\
\hline & 07-05-1977 & 87.40 & & & 11-02-1999 & 86.34 & \\
\hline & 07-10-1977 & 88.27 & & & 03-14-2000 & 75.90 & \\
\hline & 07-15-1977 & 88.79 & & & & & \\
\hline & 07-20-1977 & 88.86 & (C-36-11)18baa-1 & 5,520 & $11-18-1998$ & 73.18 & \\
\hline & 07-25-1977 & 89.03 & & & 03-16-1999 & 66.00 & \\
\hline & 07-31-1977 & 89.63 & & & 11-02-1999 & 79.16 & \\
\hline & 08-05-1977 & 89.35 & & & 03-14-2000 & 68.60 & \\
\hline
\end{tabular}


Table 2. Water levels in selected wells in Cedar Valley, Iron County, southwestern Utah-Continued

\begin{tabular}{|c|c|c|c|c|c|c|c|c|c|}
\hline Local well number & $\begin{array}{l}\text { Altitude of } \\
\text { land } \\
\text { surface } \\
\text { (feet) }\end{array}$ & Date & $\begin{array}{r}\text { Wate } \\
\text { (fe }\end{array}$ & & Local well number & $\begin{array}{l}\text { Altitude of } \\
\text { land } \\
\text { surface } \\
\text { (feet) }\end{array}$ & Date & $\begin{array}{c}\text { Water level } \\
\text { (feet) }\end{array}$ & \\
\hline \multirow[t]{7}{*}{ (C-36-11)18bca-2 } & 5,510 & $01-26-1962$ & 45 & $\mathrm{D}$ & (C-36-12)2dbc-3 & 5,490 & $05-18-1998$ & 38.45 & \\
\hline & & 05-15-1998 & 69.85 & & & & 11-18-1998 & 36.88 & \\
\hline & & $11-18-1998$ & 72.46 & & & & 03-16-1999 & 32.57 & \\
\hline & & 03-15-1999 & 65.42 & & & & 11-01-1999 & 45.69 & \\
\hline & & 11-01-1999 & 79.00 & & & & 03-14-2000 & 34.48 & \\
\hline & & $03-14-2000$ & 68.60 & & & & & & \\
\hline & & & & & (C-36-12)3aad-1 & 5,485 & 05-20-1998 & 40.00 & \\
\hline \multirow[t]{6}{*}{ (C-36-11)18bdd-1 } & 5,515 & 05-28-1994 & 67 & D & & & $11-18-1998$ & 42.14 & \\
\hline & & $11-18-1998$ & 74.82 & & & & 03-16-1999 & 37.08 & \\
\hline & & 03-15-1999 & 66.72 & & & & $03-14-2000$ & 39.24 & \\
\hline & & 11-01-1999 & 81.00 & & & & & & \\
\hline & & $03-14-2000$ & 70.06 & & $(\mathrm{C}-36-12) 3 \mathrm{aad}-2$ & 5,485 & 11-01-1999 & 52.10 & \\
\hline & & & & & & & $03-14-2000$ & 40.20 & \\
\hline \multirow[t]{5}{*}{ (C-36-11)30acc-2 } & 5,620 & 11-18-1998 & 184.86 & & & & & & \\
\hline & & 03-15-1999 & 179.90 & & (C-36-12)9aaa-1 & 5,475 & $11-08-1973$ & 14.40 & \\
\hline & & 11-01-1999 & 187.78 & & & & 03-20-1974 & 12 & $\mathrm{R}$ \\
\hline & & $03-14-2000$ & 182.14 & & & & 05-19-1998 & 31.05 & \\
\hline & & & & & & & $11-18-1998$ & 30.40 & \\
\hline \multirow[t]{6}{*}{ (C-36-11)31daa-1 } & 5,700 & 09-25-1997 & 196.37 & & & & 03-16-1999 & 26.79 & \\
\hline & & $11-17-1998$ & 194.02 & & & & 11-01-1999 & 35.59 & \\
\hline & & 03-15-1999 & 195.45 & & & & $03-14-2000$ & 30.50 & \\
\hline & & 11-01-1999 & 197.53 & & & & & & \\
\hline & & $03-14-2000$ & 198.07 & & (C-36-12)10aaa-1 & 5,470 & $12-11-1974$ & 17.34 & \\
\hline & & & & & & & 05-19-1998 & 30.24 & \\
\hline \multirow[t]{5}{*}{ (C-36-11)31daa-2 } & 5,715 & 09-25-1997 & 180.54 & & & & $11-18-1998$ & 32.14 & \\
\hline & & $11-17-1998$ & 163.67 & & & & 03-16-1999 & 26.46 & \\
\hline & & 03-15-1999 & 171.64 & & & & 11-01-1999 & 39.38 & \\
\hline & & 11-01-1999 & 208.45 & & & & $03-14-2000$ & 30.23 & \\
\hline & & 03-14-2000 & 191.37 & & & & 03-09-2001 & 35.54 & \\
\hline \multirow[t]{6}{*}{$(\mathrm{C}-36-12) 1 \mathrm{dcc}-2$} & 5,505 & 05-18-1998 & 24.48 & & (C-36-12)10dda-1 & 5,476 & $12-08-1959$ & 6.60 & \\
\hline & & $11-19-1998$ & 27.33 & & & & $03-14-1960$ & 4.28 & \\
\hline & & 03-16-1999 & 21.20 & & & & 05-03-1960 & 7.94 & \\
\hline & & 11-02-1999 & 33.17 & & & & $06-08-1960$ & 10.31 & \\
\hline & & $03-14-2000$ & 24.68 & & & & $07-11-1960$ & 11.61 & \\
\hline & & & & & & & $08-09-1960$ & 12.45 & \\
\hline \multirow[t]{9}{*}{ (C-36-12)2aac-1 } & 5,494 & $11-09-1973$ & 33.10 & & & & 09-07-1960 & 13.15 & \\
\hline & & 03-20-1974 & 21 & $\mathrm{R}$ & & & $10-31-1960$ & 10.39 & \\
\hline & & 05-19-1998 & 53.26 & & & & $12-02-1960$ & 8.75 & \\
\hline & & $11-18-1998$ & 49.86 & & & & 04-04-1961 & 6.17 & \\
\hline & & 03-16-1999 & 45.99 & & & & $05-08-1961$ & 7.84 & \\
\hline & & 03-19-1999 & 45.99 & & & & $05-25-1961$ & 11.13 & \\
\hline & & 11-01-1999 & 57.70 & & & & 03-20-1962 & 7.02 & \\
\hline & & $03-14-2000$ & 47.40 & & & & $03-25-1963$ & 7.74 & \\
\hline & & & & & & & 03-19-1964 & 9.49 & \\
\hline \multirow[t]{6}{*}{$(\mathrm{C}-36-12) 2 \mathrm{dbc}-2$} & 5,492 & 05-18-1998 & 41.03 & & & & $11-02-1964$ & 17.53 & \\
\hline & & $11-18-1998$ & 39.60 & & & & 03-19-1965 & 11.64 & \\
\hline & & 03-16-1999 & 33.82 & & & & $11-05-1965$ & 16.25 & \\
\hline & & 11-01-1999 & 48.25 & & & & 03-07-1966 & 10.42 & \\
\hline & & $03-14-2000$ & 36.38 & & & & 03-08-1967 & 12.50 & \\
\hline & & & & & & & 03-07-1968 & 11.42 & \\
\hline
\end{tabular}


Table 2. Water levels in selected wells in Cedar Valley, Iron County, southwestern Utah-Continued

\begin{tabular}{|c|c|c|c|}
\hline Local well number & $\begin{array}{c}\text { Altitude of } \\
\text { land } \\
\text { surface } \\
\text { (feet) }\end{array}$ & Date & $\begin{array}{c}\text { Water level } \\
\text { (feet) }\end{array}$ \\
\hline \multirow{51}{*}{\multicolumn{2}{|c|}{ (C-36-12)10dda-1-Continued }} & 03-17-1969 & 11.07 \\
\hline & & 03-10-1970 & 9.35 \\
\hline & & 03-11-1971 & 11.88 \\
\hline & & 03-09-1972 & 12.90 \\
\hline & & 03-14-1973 & 12.92 \\
\hline & & $07-18-1973$ & 18.50 \\
\hline & & 03-11-1974 & 9.84 \\
\hline & & 04-26-1974 & 11.43 \\
\hline & & $06-12-1974$ & 21.33 \\
\hline & & 07-11-1974 & 23.80 \\
\hline & & 08-08-1974 & 23.34 \\
\hline & & 09-11-1974 & 23.47 \\
\hline & & $10-08-1974$ & 20.80 \\
\hline & & 11-11-1974 & 17.80 \\
\hline & & $12-03-1974$ & 16.23 \\
\hline & & 01-07-1975 & 14.82 \\
\hline & & 02-10-1975 & 13.33 \\
\hline & & 03-04-1975 & 13.13 \\
\hline & & 04-14-1975 & 12.00 \\
\hline & & 05-07-1975 & 12.14 \\
\hline & & 06-04-1975 & 17.23 \\
\hline & & 07-07-1975 & 22.25 \\
\hline & & $08-12-1975$ & 26.46 \\
\hline & & 09-08-1975 & 27.81 \\
\hline & & $10-04-1975$ & 24.42 \\
\hline & & 11-06-1975 & 21.23 \\
\hline & & $12-06-1975$ & 17.93 \\
\hline & & 03-01-1976 & 14.15 \\
\hline & & $10-07-1976$ & 28.37 \\
\hline & & 03-01-1977 & 16.83 \\
\hline & & 10-06-1977 & 29.84 \\
\hline & & 02-28-1978 & 19.93 \\
\hline & & 03-15-1978 & 19.58 \\
\hline & & 10-03-1978 & 27.65 \\
\hline & & 03-08-1979 & 18.94 \\
\hline & & 10-01-1979 & 26.90 \\
\hline & & 03-10-1980 & 15.83 \\
\hline & & 03-04-1981 & 12.87 \\
\hline & & 03-02-1982 & 12.36 \\
\hline & & 03-02-1983 & 10.75 \\
\hline & & 03-05-1984 & 7.29 \\
\hline & & 03-05-1985 & 5.55 \\
\hline & & 03-03-1986 & 5.74 \\
\hline & & 03-12-1987 & 5.09 \\
\hline & & 03-01-1988 & 5.30 \\
\hline & & 03-08-1989 & 3.40 \\
\hline & & 03-10-1992 & 14.30 \\
\hline & & 03-08-1993 & 15.47 \\
\hline & & 03-09-1994 & 14.13 \\
\hline & & 03-09-1995 & 18.10 \\
\hline & & 03-08-1996 & 16.55 \\
\hline
\end{tabular}

\begin{tabular}{|c|c|c|c|}
\hline Local well number & $\begin{array}{l}\text { Altitude of } \\
\text { land } \\
\text { surface } \\
\text { (feet) }\end{array}$ & Date & $\begin{array}{l}\text { Water level } \\
\text { (feet) }\end{array}$ \\
\hline \multirow[t]{51}{*}{$(\mathrm{C}-36-12) 12 \mathrm{dba}-1$} & 5,509 & 11-01-1936 & 17.00 \\
\hline & & $11-23-1936$ & 17.61 \\
\hline & & $12-22-1936$ & 17.85 \\
\hline & & 01-23-1937 & 17.80 \\
\hline & & 02-20-1937 & 18.70 \\
\hline & & 03-20-1937 & 14.44 \\
\hline & & 04-17-1937 & 13.70 \\
\hline & & 09-27-1937 & 19.12 \\
\hline & & $10-30-1937$ & 17.79 \\
\hline & & $11-27-1937$ & 15.74 \\
\hline & & $12-18-1937$ & 15.81 \\
\hline & & 01-29-1938 & 14.70 \\
\hline & & 02-26-1938 & 14.43 \\
\hline & & 03-26-1938 & 14.17 \\
\hline & & 04-23-1938 & 13.70 \\
\hline & & $05-21-1938$ & 13.39 \\
\hline & & 06-18-1938 & 13.78 \\
\hline & & $07-12-1938$ & 17.54 \\
\hline & & 09-27-1938 & 16.88 \\
\hline & & $10-30-1938$ & 15.81 \\
\hline & & $11-12-1938$ & 15.44 \\
\hline & & $12-02-1938$ & 14.26 \\
\hline & & 01-03-1939 & 12.49 \\
\hline & & 02-01-1939 & 12.90 \\
\hline & & 03-01-1939 & 12.52 \\
\hline & & 04-03-1939 & 11.79 \\
\hline & & 09-27-1939 & 17.75 \\
\hline & & $10-21-1939$ & 16.89 \\
\hline & & $12-11-1939$ & 15.34 \\
\hline & & 01-17-1940 & 14.76 \\
\hline & & $02-15-1940$ & 14.25 \\
\hline & & 03-16-1940 & 14.25 \\
\hline & & 04-08-1940 & 14.89 \\
\hline & & $12-02-1940$ & 17.65 \\
\hline & & 03-23-1941 & 15.91 \\
\hline & & $12-02-1941$ & 14.26 \\
\hline & & 03-16-1942 & 12.25 \\
\hline & & 08-07-1942 & 15.78 \\
\hline & & $12-16-1942$ & 12.42 \\
\hline & & 03-23-1943 & 10.35 \\
\hline & & $12-11-1943$ & 13.50 \\
\hline & & $12-07-1944$ & 13.43 \\
\hline & & 03-30-1945 & 12.28 \\
\hline & & $12-08-1945$ & 13.89 \\
\hline & & 03-18-1946 & 12.76 \\
\hline & & $12-14-1946$ & 15.24 \\
\hline & & 03-24-1947 & 14.36 \\
\hline & & $12-07-1947$ & 15.74 \\
\hline & & 03-11-1948 & 16.40 \\
\hline & & $12-06-1948$ & 16.49 \\
\hline & & 04-30-1949 & 13.32 \\
\hline
\end{tabular}


Table 2. Water levels in selected wells in Cedar Valley, Iron County, southwestern Utah-Continued

\begin{tabular}{|c|c|c|c|c|c|c|}
\hline Local well number & $\begin{array}{l}\text { Altitude of } \\
\text { land } \\
\text { surface } \\
\text { (feet) }\end{array}$ & Date & $\begin{array}{c}\text { Water level } \\
\text { (feet) }\end{array}$ & $\begin{array}{c}\text { Altitude of } \\
\text { land } \\
\text { surface } \\
\text { (feet) }\end{array}$ & Date & $\begin{array}{l}\text { Water level } \\
\text { (feet) }\end{array}$ \\
\hline \multirow{51}{*}{\multicolumn{2}{|c|}{ (C-36-12)12dba-1—Continued }} & 08-31-1949 & 19.79 & \multirow[t]{51}{*}{ (C-36-12)12dba-1-Continued } & $12-08-1959$ & 32.47 \\
\hline & & $10-31-1949$ & 19.56 & & $03-15-1960$ & 28.69 \\
\hline & & 11-29-1949 & 14.38 & & $10-31-1960$ & 38.55 \\
\hline & & 12-07-1949 & 13.83 & & $12-02-1960$ & 35.65 \\
\hline & & 01-31-1950 & 12.71 & & 04-04-1961 & 31.00 \\
\hline & & 03-01-1950 & 12.34 & & $10-31-1961$ & 41.78 \\
\hline & & 04-01-1950 & 11.58 & & 03-20-1962 & 32.03 \\
\hline & & $05-02-1950$ & 11.46 & & $03-25-1963$ & 32.67 \\
\hline & & $10-27-1950$ & 21.64 & & $10-10-1963$ & 49.11 \\
\hline & & $12-30-1950$ & 16.68 & & 03-23-1964 & 34.68 \\
\hline & & 01-31-1951 & 15.76 & & 03-19-1965 & 37.41 \\
\hline & & 03-02-1951 & 15.58 & & $11-05-1965$ & 34.50 \\
\hline & & 03-21-1951 & 15.17 & & 03-07-1966 & 34.64 \\
\hline & & 03-31-1951 & 15.72 & & $09-27-1966$ & 57.26 \\
\hline & & $10-17-1951$ & 23.50 & & 03-08-1967 & 35.40 \\
\hline & & $10-31-1951$ & 22.77 & & $10-06-1967$ & 43.00 \\
\hline & & $11-30-1951$ & 21.60 & & 03-07-1968 & 34.26 \\
\hline & & $12-07-1951$ & 20.65 & & $10-08-1968$ & 44.32 \\
\hline & & 01-03-1952 & 17.00 & & 03-17-1969 & 30.85 \\
\hline & & 02-05-1952 & 14.80 & & $10-08-1969$ & 41.30 \\
\hline & & 03-05-1952 & 13.66 & & 03-11-1970 & 27.38 \\
\hline & & 04-04-1952 & 17.56 & & $10-08-1970$ & 51.34 \\
\hline & & 05-05-1952 & 15.76 & & 03-10-1971 & 31.30 \\
\hline & & 06-04-1952 & 16.10 & & 03-09-1972 & 31.89 \\
\hline & & $10-07-1952$ & 21.84 & & $10-04-1972$ & 50.55 \\
\hline & & $11-06-1952$ & 21.09 & & 03-14-1973 & 32.22 \\
\hline & & $12-04-1952$ & 18.65 & & $10-05-1973$ & 37.30 \\
\hline & & $12-05-1952$ & 19.07 & & 03-11-1974 & 25.45 \\
\hline & & 01-03-1953 & 17.68 & & $10-08-1974$ & 52.33 \\
\hline & & 02-07-1953 & 16.80 & & 03-05-1975 & 31.59 \\
\hline & & 03-05-1953 & 16.36 & & 03-01-1976 & 30.75 \\
\hline & & 03-13-1953 & 16.22 & & $10-07-1976$ & 53.34 \\
\hline & & 04-10-1953 & 20.75 & & 03-01-1977 & 36.53 \\
\hline & & $11-02-1953$ & 23.60 & & $10-05-1977$ & 62.38 \\
\hline & & $12-03-1953$ & 19.90 & & $02-28-1978$ & 42.77 \\
\hline & & $12-07-1953$ & 22.35 & & 03-15-1978 & 42.28 \\
\hline & & 03-02-1954 & 19.84 & & 03-08-1979 & 40.08 \\
\hline & & 01-05-1955 & 22.86 & & 03-10-1980 & 34.95 \\
\hline & & 02-03-1955 & 21.95 & & 03-03-1981 & 30.23 \\
\hline & & 03-04-1955 & 21.46 & & $10-01-1981$ & 43.61 \\
\hline & & 03-19-1955 & 21.13 & & 03-02-1982 & 30.65 \\
\hline & & 04-05-1955 & 21.04 & & $10-01-1982$ & 29.01 \\
\hline & & $12-02-1955$ & 31.93 & & 03-02-1983 & 28.25 \\
\hline & & 03-15-1956 & 23.37 & & $09-27-1983$ & 35.62 \\
\hline & & $12-12-1956$ & 29.50 & & 09-26-1984 & 30.81 \\
\hline & & 03-11-1957 & 26.30 & & 03-05-1985 & 22.57 \\
\hline & & $10-23-1957$ & 33.78 & & $10-24-1985$ & 27.83 \\
\hline & & $12-04-1957$ & 30.57 & & 03-03-1986 & 21.24 \\
\hline & & 03-17-1958 & 26.09 & & 09-29-1986 & 21.28 \\
\hline & & $10-24-1958$ & 34.72 & & 03-12-1987 & 21.49 \\
\hline & & 04-08-1959 & 26.53 & & 09-17-1987 & 27.08 \\
\hline
\end{tabular}


Table 2. Water levels in selected wells in Cedar Valley, Iron County, southwestern Utah-Continued

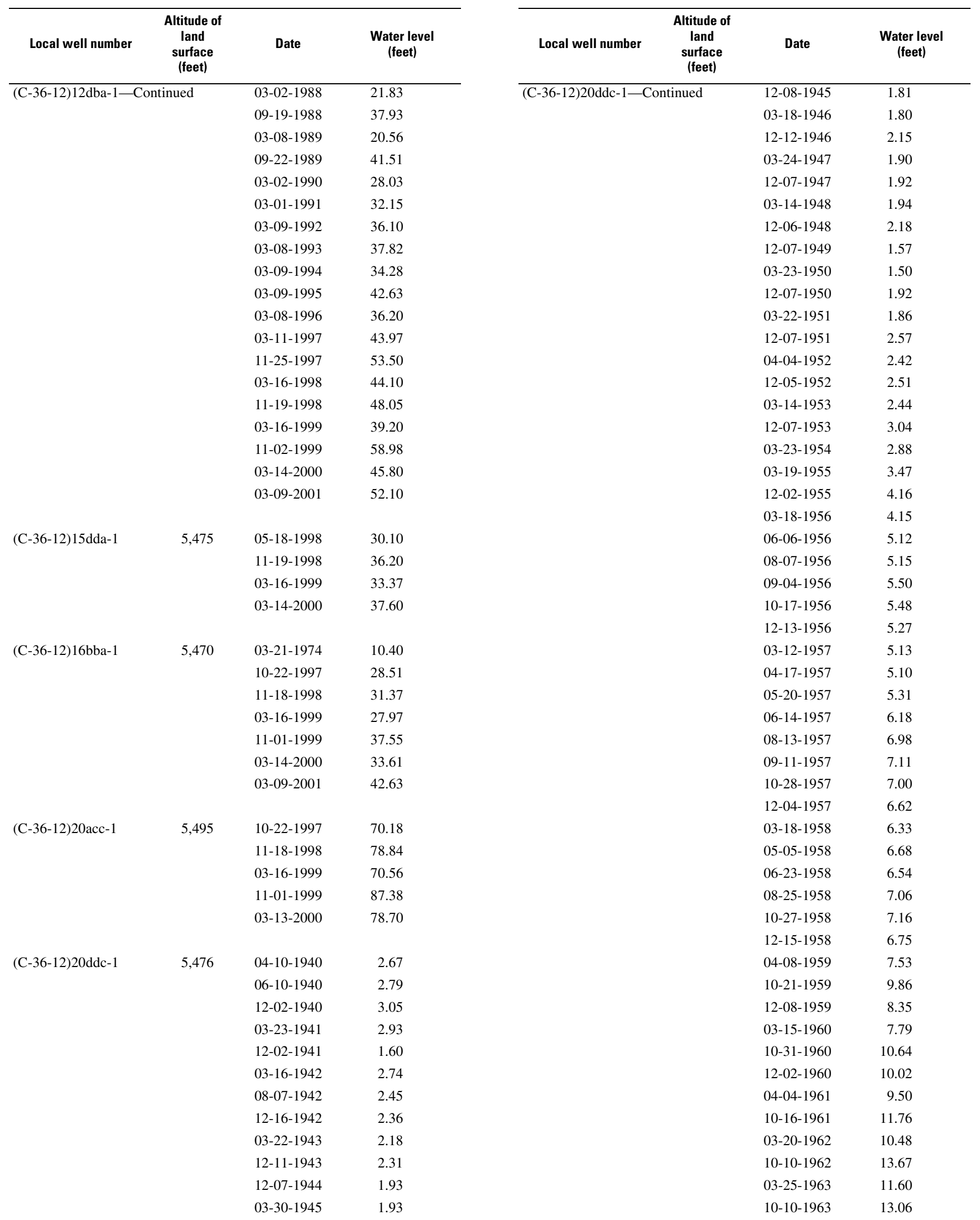


Table 2. Water levels in selected wells in Cedar Valley, Iron County, southwestern Utah-Continued

\begin{tabular}{|c|c|c|c|c|c|c|c|}
\hline Local well number & $\begin{array}{c}\text { Altitude of } \\
\text { land } \\
\text { surface } \\
\text { (feet) }\end{array}$ & Date & $\begin{array}{c}\text { Water level } \\
\text { (feet) }\end{array}$ & Local well number & $\begin{array}{l}\text { Altitude of } \\
\text { land } \\
\text { surface } \\
\text { (feet) }\end{array}$ & Date & $\begin{array}{c}\text { Water level } \\
\text { (feet) }\end{array}$ \\
\hline \multirow{51}{*}{\multicolumn{2}{|c|}{ (C-36-12)20ddc-1—Continued }} & 03-23-1964 & 12.72 & \multicolumn{2}{|c|}{ (C-36-12)20ddc-1—Continued } & $10-01-1980$ & 28.66 \\
\hline & & 11-02-1964 & 16.81 & & & 03-04-1981 & 24.83 \\
\hline & & 03-19-1965 & 14.83 & & & 03-02-1982 & 23.28 \\
\hline & & $11-05-1965$ & 16.58 & & & $10-01-1982$ & 24.69 \\
\hline & & 03-07-1966 & 14.94 & & & 03-02-1983 & 22.01 \\
\hline & & $09-27-1966$ & 19.35 & & & $09-27-1983$ & 23.20 \\
\hline & & 03-08-1967 & 16.72 & & & 03-05-1984 & 20.39 \\
\hline & & $10-06-1967$ & 20.25 & & & 09-26-1984 & 21.95 \\
\hline & & 03-07-1968 & 17.13 & & & 03-05-1985 & 19.12 \\
\hline & & $10-08-1968$ & 23.55 & & & $10-21-1985$ & 21.20 \\
\hline & & 03-19-1969 & 17.93 & & & 03-03-1986 & 18.55 \\
\hline & & $10-07-1969$ & 21.20 & & & 09-29-1986 & 20.76 \\
\hline & & 03-10-1970 & 18.05 & & & 03-12-1987 & 19.84 \\
\hline & & $10-08-1970$ & 23.68 & & & 09-17-1987 & 19.77 \\
\hline & & 03-10-1971 & 19.92 & & & 03-01-1988 & 19.53 \\
\hline & & 09-30-1971 & 26.48 & & & 09-19-1988 & 19.80 \\
\hline & & 03-10-1972 & 22.84 & & & 03-08-1989 & 19.02 \\
\hline & & 03-14-1973 & 22.23 & & & 09-22-1989 & 23.70 \\
\hline & & 07-18-1973 & 25.83 & & & 03-07-1990 & 21.48 \\
\hline & & $10-05-1973$ & 29.85 & & & 09-22-1990 & 27.00 \\
\hline & & 03-11-1974 & 21.40 & & & 03-01-1991 & 21.91 \\
\hline & & 04-26-1974 & 24.23 & & & 09-18-1991 & 28.02 \\
\hline & & 06-12-1974 & 27.68 & & & 03-10-1992 & 23.77 \\
\hline & & 07-11-1974 & 27.08 & & & 09-30-1992 & 33.22 \\
\hline & & 08-08-1974 & 27.80 & & & 03-08-1993 & 25.28 \\
\hline & & 09-11-1974 & 30.02 & & & 03-09-1994 & 26.52 \\
\hline & & 10-08-1974 & 30.01 & & & 03-09-1995 & 30.90 \\
\hline & & 11-11-1974 & 27.49 & & & 03-08-1996 & 30.97 \\
\hline & & 12-03-1974 & 26.37 & & & 03-04-1997 & 34.32 \\
\hline & & 01-07-1975 & 25.23 & & & 03-16-1998 & 34.67 \\
\hline & & 02-10-1975 & 24.40 & & & & \\
\hline & & 03-04-1975 & 24.40 & (C-36-12)21 cbb-1 & 5,470 & 09-25-1997 & 42.90 \\
\hline & & 04-14-1975 & 23.41 & & & 07-13-1998 & 65.08 \\
\hline & & 05-07-1975 & 26.44 & & & 11-17-1998 & 61.44 \\
\hline & & 06-04-1975 & 28.02 & & & 03-15-1999 & 38.70 \\
\hline & & 07-07-1975 & 37.88 & & & 11-01-1999 & 55.36 \\
\hline & & 08-12-1975 & 30.39 & & & 03-14-2000 & 61.58 \\
\hline & & 09-08-1975 & 31.99 & & & 04-03-2000 & 52.01 \\
\hline & & 10-04-1975 & 32.51 & & & 03-09-2001 & 53.10 \\
\hline & & 11-06-1975 & 29.31 & & & & \\
\hline & & 12-06-1975 & 27.60 & (C-36-12)25bdd-1 & 5,540 & 03-15-1957 & 65.14 \\
\hline & & 03-01-1976 & 25.11 & & & 04-18-1957 & 64.97 \\
\hline & & $10-07-1976$ & 31.62 & & & 08-13-1957 & 66.45 \\
\hline & & 03-01-1977 & 27.94 & & & $10-28-1957$ & 67.46 \\
\hline & & 10-06-1977 & 33.95 & & & $12-04-1957$ & 67.20 \\
\hline & & 02-28-1978 & 28.44 & & & $03-27-1958$ & 66.25 \\
\hline & & 03-15-1978 & 27.97 & & & 08-06-1958 & 67.14 \\
\hline & & $10-02-1978$ & 32.06 & & & 09-16-1958 & 68.54 \\
\hline & & 03-12-1979 & 28.38 & & & $10-27-1958$ & 64.50 \\
\hline & & 10-01-1979 & 30.48 & & & $12-22-1958$ & 68.07 \\
\hline & & 03-10-1980 & 27.12 & & & 04-09-1959 & 67.04 \\
\hline
\end{tabular}


Table 2. Water levels in selected wells in Cedar Valley, Iron County, southwestern Utah-Continued

\begin{tabular}{|c|c|c|c|c|c|c|c|}
\hline Local well number & $\begin{array}{c}\text { Altitude of } \\
\text { land } \\
\text { surface } \\
\text { (feet) }\end{array}$ & Date & $\begin{array}{c}\text { Water level } \\
\text { (feet) }\end{array}$ & Local well number & $\begin{array}{c}\text { Altitude of } \\
\text { land } \\
\text { surface } \\
\text { (feet) }\end{array}$ & Date & $\begin{array}{c}\text { Water level } \\
\text { (feet) }\end{array}$ \\
\hline \multirow{50}{*}{\multicolumn{2}{|c|}{ (C-36-12)25bdd-1-Continued }} & 05-04-1959 & 68.18 & (C-36-12)25cda-1 & 5,550 & 09-29-1997 & 105.40 \\
\hline & & 06-01-1959 & 68.64 & & & $11-18-1998$ & 106.12 \\
\hline & & 07-02-1959 & 69.55 & & & 03-15-1999 & 103.50 \\
\hline & & $10-21-1959$ & 71.82 & & & 05-19-1999 & 103.89 \\
\hline & & 12-08-1959 & 71.11 & & & 05-21-1999 & 103.94 \\
\hline & & 03-15-1960 & 70.00 & & & 05-22-1999 & 104.05 \\
\hline & & $05-02-1960$ & 70.76 & & & 05-23-1999 & 104.18 \\
\hline & & 06-08-1960 & 71.69 & & & 05-24-1999 & 104.24 \\
\hline & & 07-11-1960 & 72.47 & & & 05-25-1999 & 104.30 \\
\hline & & 08-09-1960 & 73.01 & & & 05-26-1999 & 104.34 \\
\hline & & 09-08-1960 & 74.75 & & & 05-26-1999 & 104.35 \\
\hline & & $10-31-1960$ & 74.98 & & & 11-02-1999 & 110.72 \\
\hline & & $12-05-1960$ & 74.05 & & & 03-13-2000 & 107.93 \\
\hline & & 04-04-1961 & 72.13 & & & & \\
\hline & & 03-20-1962 & 73.41 & (C-36-12)28ccc-1 & 5,465 & 09-24-1997 & 28.41 \\
\hline & & 03-25-1963 & 76.98 & & & 11-17-1998 & 30.05 \\
\hline & & 03-19-1964 & 76.29 & & & 03-15-1999 & 27.59 \\
\hline & & 03-19-1965 & 79.13 & & & 11-01-1999 & 34.28 \\
\hline & & $11-05-1965$ & 86.81 & & & 03-13-2000 & 32.06 \\
\hline & & 03-07-1966 & 80.02 & & & & \\
\hline & & 03-08-1967 & 82.00 & $(\mathrm{C}-36-12) 32 \mathrm{dcc}-1$ & 5,498 & 10-05-1977 & 75.29 \\
\hline & & 03-12-1968 & 81.37 & & & 02-28-1978 & 58.51 \\
\hline & & 03-20-1969 & 82.78 & & & 03-20-1978 & 54.99 \\
\hline & & 03-11-1970 & 80.65 & & & 03-12-1979 & 53.45 \\
\hline & & 04-01-1971 & 81.00 & & & 10-01-1979 & 56.52 \\
\hline & & 03-09-1972 & 88.32 & & & 03-10-1980 & 51.00 \\
\hline & & 03-14-1974 & 80.90 & & & 03-04-1981 & 48.82 \\
\hline & & 03-05-1975 & 82.54 & & & 03-02-1982 & 45.92 \\
\hline & & 03-12-1976 & 86.10 & & & 03-02-1983 & 44.35 \\
\hline & & 03-01-1977 & 90.75 & & & 03-05-1984 & 42.75 \\
\hline & & 03-12-1978 & 98.79 & & & 03-05-1985 & 41.79 \\
\hline & & 03-17-1978 & 96.75 & & & 03-03-1986 & 40.90 \\
\hline & & 03-02-1979 & 98.79 & & & 03-12-1987 & 39.05 \\
\hline & & $10-01-1979$ & 97.24 & & & 03-01-1988 & 39.48 \\
\hline & & 03-10-1980 & 92.96 & & & 03-08-1989 & 39.38 \\
\hline & & 03-04-1981 & 84.70 & & & 03-07-1990 & 42.19 \\
\hline & & 03-02-1982 & 89.40 & & & 03-01-1991 & 46.18 \\
\hline & & 03-08-1983 & 82.95 & & & 03-10-1992 & 47.42 \\
\hline & & 03-05-1985 & 74.94 & & & 03-08-1993 & 48.10 \\
\hline & & 03-03-1986 & 72.05 & & & 03-09-1994 & 47.92 \\
\hline & & 03-09-1987 & 71.75 & & & 03-09-1995 & 51.30 \\
\hline & & 05-19-1999 & 90.46 & & & 03-08-1996 & 50.45 \\
\hline & & 05-21-1999 & 90.76 & & & 03-04-1997 & 56.85 \\
\hline & & 05-22-1999 & 90.82 & & & $11-25-1997$ & 69.43 \\
\hline & & 05-23-1999 & 90.95 & & & 03-17-1998 & 59.60 \\
\hline & & 05-24-1999 & 90.85 & & & 11-17-1998 & 61.22 \\
\hline & & 05-25-1999 & 90.92 & & & 03-15-1999 & 58.63 \\
\hline & & 05-26-1999 & 91.33 & & & 11-01-1999 & 69.35 \\
\hline & & 11-02-1999 & 94.38 & & & $03-13-2000$ & 62.78 \\
\hline & & 03-13-2000 & 93.52 & & & 03-09-2001 & 66.90 \\
\hline
\end{tabular}


Table 2. Water levels in selected wells in Cedar Valley, Iron County, southwestern Utah-Continued

\begin{tabular}{|c|c|c|c|c|c|c|c|c|c|}
\hline Local well number & $\begin{array}{c}\text { Altitude of } \\
\text { land } \\
\text { surface } \\
\text { (feet) }\end{array}$ & Date & $\begin{array}{r}\text { Wate } \\
\text { (fe }\end{array}$ & & Local well number & $\begin{array}{l}\text { Altitude of } \\
\text { land } \\
\text { surface } \\
\text { (feet) }\end{array}$ & Date & $\begin{array}{l}\text { Water } \\
\text { (fe }\end{array}$ & \\
\hline (C-36-12)32dcd-1 & 5,482 & $10-03-1973$ & 51 & $\mathrm{R}$ & $(\mathrm{C}-37-12) 5 \mathrm{acc}-1-\mathrm{Co}$ & nued & 03-22-1974 & 61 & $\mathrm{R}$ \\
\hline & & 03-20-1974 & 36 & $\mathrm{R}$ & & & 09-24-1997 & 71.70 & \\
\hline & & 09-25-1997 & 51.65 & & & & $11-17-1998$ & 69.22 & \\
\hline & & 11-17-1998 & 49.44 & & & & 03-15-1999 & 66.92 & \\
\hline & & 03-15-1999 & 47.00 & & & & 11-01-1999 & 82.36 & \\
\hline & & 11-01-1999 & 56.07 & & & & $03-13-2000$ & 70.52 & \\
\hline & & 03-13-2000 & 50.90 & & & & & & \\
\hline & & & & & (C-37-12)5acc-2 & 5,505 & $11-17-1998$ & 67.28 & \\
\hline (C-36-12)33bcc-1 & 5,465 & 09-24-1997 & 34.70 & & & & & & \\
\hline & & $11-17-1998$ & 29.97 & & (C-37-12)5bbb-1 & 5,532 & $10-11-1973$ & 92.38 & \\
\hline & & 03-15-1999 & 27.48 & & & & 03-20-1974 & 79 & $\mathrm{R}$ \\
\hline & & 11-01-1999 & 35.08 & & & & 03-17-1998 & 100.27 & \\
\hline & & $03-13-2000$ & 31.60 & & & & $11-17-1998$ & 97.30 & \\
\hline & & & & & & & 03-15-1999 & 98.00 & \\
\hline (C-36-12)35adc-1 & 5,510 & $11-20-1977$ & 75.16 & & & & 11-01-1999 & 114.47 & \\
\hline & & 03-17-1978 & 69.15 & & & & $03-13-2000$ & 97.35 & \\
\hline & & 03-12-1979 & 68.20 & & & & & & \\
\hline & & 03-04-1981 & 59.61 & & (C-37-12)5bcb-1 & 5,542 & $05-15-1960$ & 70 & $\mathrm{D}$ \\
\hline & & 03-05-1982 & 58.00 & & & & 03-17-1998 & 108.57 & \\
\hline & & 03-08-1983 & 57.32 & & & & $11-17-1998$ & 104.38 & \\
\hline & & 03-05-1985 & 48.55 & & & & 03-15-1999 & 107.77 & \\
\hline & & 03-03-1986 & 48.13 & & & & 11-01-1999 & 120.58 & \\
\hline & & 03-09-1987 & 47.85 & & & & $03-13-2000$ & 104.57 & \\
\hline & & 03-07-1988 & 47.90 & & & & & & \\
\hline & & 03-08-1989 & 47.73 & & (C-37-12)9acc-1 & 5,490 & 07-18-1959 & 16 & $\mathrm{D}$ \\
\hline & & 03-05-1990 & 48.42 & & & & $10-22-1997$ & 47.28 & \\
\hline & & 03-01-1991 & 51.11 & & & & 03-17-1998 & 44.55 & \\
\hline & & 03-10-1992 & 54.18 & & & & $11-17-1998$ & 55.35 & \\
\hline & & 03-08-1993 & 57.00 & & & & 03-12-1999 & 44.88 & \\
\hline & & 03-09-1994 & 55.84 & & & & 03-20-2001 & 50.55 & \\
\hline & & 03-09-1995 & 61.17 & & & & & & \\
\hline & & 03-08-1996 & 55.59 & & (C-37-12)11 aaa-1 & 5,490 & 09-29-1953 & 25 & D \\
\hline & & 03-03-1997 & 61.92 & & & & $12-07-1953$ & 19.14 & \\
\hline & & $11-25-1997$ & 65.64 & & & & 03-23-1954 & 18.11 & \\
\hline & & 03-16-1998 & 63.22 & & & & 03-19-1955 & 20.14 & \\
\hline & & 11-18-1998 & 65.38 & & & & $12-02-1955$ & 22.98 & \\
\hline & & 03-15-1999 & 62.12 & & & & 03-18-1956 & 20.97 & \\
\hline & & 11-02-1999 & 70.46 & & & & 06-06-1956 & 31.44 & \\
\hline & & $03-13-2000$ & 68.25 & & & & 08-07-1956 & 33.05 & \\
\hline & & 03-09-2001 & 71.60 & & & & 09-04-1956 & 34.79 & \\
\hline & & & & & & & $10-17-1956$ & 29.79 & \\
\hline (C-36-12)36ada-1 & 5,604 & $11-14-1973$ & 153.90 & & & & $12-17-1956$ & 26.84 & \\
\hline & & 03-20-1974 & 143 & $\mathrm{R}$ & & & 03-15-1957 & 24.71 & \\
\hline & & 09-29-1997 & 167.85 & & & & 04-18-1957 & 24.24 & \\
\hline & & $11-18-1998$ & 154.75 & & & & 06-14-1957 & 30.53 & \\
\hline & & 03-15-1999 & 152.30 & & & & $10-28-1957$ & 30.65 & \\
\hline & & 11-02-1999 & 164.16 & & & & $12-04-1957$ & 26.46 & \\
\hline & & 03-13-2000 & 159.02 & & & & 03-18-1958 & 24.87 & \\
\hline & & & & & & & 05-07-1958 & 24.27 & \\
\hline (C-37-12)5acc-1 & 5,504 & 06-24-1959 & 36 & $\mathrm{D}$ & & & $06-23-1958$ & 26.89 & \\
\hline & & $07-17-1973$ & 63.66 & & & & 08-06-1958 & 30.71 & \\
\hline
\end{tabular}


Table 2. Water levels in selected wells in Cedar Valley, Iron County, southwestern Utah-Continued

\begin{tabular}{|c|c|c|c|c|c|c|c|c|}
\hline Local well number & $\begin{array}{c}\text { Altitude of } \\
\text { land } \\
\text { surface } \\
\text { (feet) }\end{array}$ & Date & $\begin{array}{c}\text { Water level } \\
\text { (feet) }\end{array}$ & Local well number & $\begin{array}{l}\text { Altitude of } \\
\text { land } \\
\text { surface } \\
\text { (feet) }\end{array}$ & Date & $\begin{array}{c}\text { Water level } \\
\text { (feet) }\end{array}$ & \\
\hline \multirow{51}{*}{\multicolumn{2}{|c|}{ (C-37-12)11aaa-1-Continued }} & 09-16-1958 & 31.84 & $\begin{array}{l}\text { (C-37-12)11aab-1 } \\
\text { (a) }\end{array}$ & 5,490 & 06-12-1995 & 30 & $\mathrm{D}$ \\
\hline & & $10-27-1958$ & 30.20 & & & 09-24-1997 & 51.97 & \\
\hline & & $12-15-1958$ & 27.61 & & & $11-17-1998$ & 52.02 & \\
\hline & & 04-09-1959 & 25.49 & & & 03-12-1999 & 49.77 & \\
\hline & & 05-04-1959 & 26.00 & & & 11-01-1999 & 60.75 & \\
\hline & & 06-01-1959 & 32.04 & & & $03-13-2000$ & 54.33 & \\
\hline & & 07-02-1959 & 35.80 & & & & & \\
\hline & & 08-02-1959 & 36.55 & $(\mathrm{C}-37-12) 11 \mathrm{dac}-1$ & 5,495 & 11-01-1999 & 79.10 & \\
\hline & & 09-09-1959 & 35.80 & & & 03-13-2000 & 73.10 & \\
\hline & & $10-21-1959$ & 32.00 & & & & & \\
\hline & & $12-08-1959$ & 29.90 & $(\mathrm{C}-37-12) 14 \mathrm{abc}-1$ & 5,480 & $10-21-1959$ & 25.50 & \\
\hline & & $03-15-1960$ & 27.70 & & & $12-08-1959$ & 24.14 & \\
\hline & & $05-02-1960$ & 27.64 & & & $03-15-1960$ & 20.02 & \\
\hline & & 07-11-1960 & 32.22 & & & $10-31-1960$ & 30.70 & \\
\hline & & 08-09-1960 & 38.35 & & & $12-02-1960$ & 26.77 & \\
\hline & & 09-08-1960 & 36.98 & & & 04-04-1961 & 27.20 & \\
\hline & & $10-31-1960$ & 35.18 & & & $03-25-1963$ & 26.08 & \\
\hline & & $12-02-1960$ & 32.06 & & & $10-10-1963$ & 31.91 & \\
\hline & & 04-04-1961 & 29.37 & & & 03-19-1964 & 28.10 & \\
\hline & & 05-09-1961 & 29.10 & & & 03-19-1965 & 30.18 & \\
\hline & & 05-25-1961 & 35.80 & & & 03-07-1966 & 31.07 & \\
\hline & & 03-20-1962 & 32.07 & & & $03-08-1967$ & 31.65 & \\
\hline & & $03-25-1963$ & 31.95 & & & 03-07-1968 & 30.90 & \\
\hline & & 03-19-1964 & 33.88 & & & 03-17-1969 & 31.50 & \\
\hline & & 11-03-1964 & 46.25 & & & 03-10-1970 & 30.80 & \\
\hline & & 03-19-1965 & 37.70 & & & $04-01-1971$ & 33.20 & \\
\hline & & 11-05-1965 & 41.05 & & & 03-09-1972 & 35.03 & \\
\hline & & 03-07-1966 & 35.22 & & & 03-05-1973 & 37.10 & \\
\hline & & 03-08-1967 & 38.34 & & & 03-11-1974 & 33.10 & \\
\hline & & 03-07-1968 & 38.00 & & & 03-05-1975 & 36.45 & \\
\hline & & 03-17-1969 & 38.60 & & & 03-01-1976 & 38.56 & \\
\hline & & 03-10-1970 & 37.20 & & & $10-07-1976$ & 44.61 & \\
\hline & & 03-08-1971 & 41.55 & & & 03-01-1977 & 40.74 & \\
\hline & & 03-09-1972 & 42.54 & & & $10-06-1977$ & 54.24 & \\
\hline & & 03-05-1973 & 44.58 & & & 03-03-1978 & 44.07 & \\
\hline & & 03-14-1974 & 41.28 & & & 03-16-1978 & 43.98 & \\
\hline & & 03-05-1975 & 46.30 & & & 03-06-1979 & 42.13 & \\
\hline & & 03-01-1976 & 47.74 & & & 03-07-1980 & 38.07 & \\
\hline & & 03-01-1977 & 48.71 & & & 03-04-1981 & 34.80 & \\
\hline & & 03-17-1978 & 52.47 & & & 03-01-1982 & 34.38 & \\
\hline & & 03-06-1979 & 52.10 & & & $03-08-1983$ & 33.18 & \\
\hline & & 03-04-1981 & 47.89 & & & 03-07-1984 & 30.03 & \\
\hline & & 03-01-1982 & 46.45 & & & 03-04-1985 & 28.28 & \\
\hline & & 03-08-1983 & 42.10 & & & 03-03-1986 & 27.65 & \\
\hline & & 09-25-1997 & 50.48 & & & 03-09-1987 & 26.89 & \\
\hline & & $11-17-1998$ & 52.35 & & & 03-01-1988 & 26.38 & \\
\hline & & 03-12-1999 & 49.35 & & & 03-08-1989 & 25.38 & \\
\hline & & 08-23-1999 & 62.83 & & & 03-05-1990 & 27.81 & \\
\hline & & 11-01-1999 & 60.22 & & & 03-01-1991 & 32.70 & \\
\hline & & $03-13-2000$ & 53.91 & & & 03-10-1992 & 31.65 & \\
\hline & & 03-09-2001 & 58.80 & & & 03-08-1993 & 33.09 & \\
\hline
\end{tabular}


Table 2. Water levels in selected wells in Cedar Valley, Iron County, southwestern Utah-Continued

\begin{tabular}{|c|c|c|c|c|c|c|c|}
\hline Local well number & $\begin{array}{l}\text { Altitude of } \\
\text { land } \\
\text { surface } \\
\text { (feet) }\end{array}$ & Date & $\begin{array}{l}\text { Water level } \\
\text { (feet) }\end{array}$ & & :al well number $\begin{array}{c}\text { Altitude of } \\
\text { land } \\
\text { surface } \\
\text { (feet) }\end{array}$ & Date & $\begin{array}{c}\text { Water level } \\
\text { (feet) }\end{array}$ \\
\hline (C-37-12)14abc-1-C & tinued & 03-09-1994 & 32.97 & & (C-37-12)23acb-1-Continued & 04-04-1939 & 48.14 \\
\hline & & 03-09-1995 & 35.73 & & & 05-06-1939 & 59.50 \\
\hline & & 03-08-1996 & 35.10 & & & 06-10-1939 & 72.58 \\
\hline & & 03-03-1997 & 38.92 & & & 07-09-1939 & 78.70 \\
\hline & & $11-25-1997$ & 43.83 & & & 08-15-1939 & 51.78 \\
\hline & & 03-17-1998 & 44.08 & & & 09-27-1939 & 51.42 \\
\hline & & $11-17-1998$ & 45.63 & & & 10-21-1939 & 51.28 \\
\hline & & 03-12-1999 & 42.26 & & & 12-09-1939 & 50.77 \\
\hline & & 11-01-1999 & 51.90 & & & 01-18-1940 & 50.50 \\
\hline & & $03-13-2000$ & 46.37 & & & 03-22-1940 & 50.25 \\
\hline & & 03-09-2001 & 49.71 & & & 04-09-1940 & 50.23 \\
\hline & & & & & & $12-02-1940$ & 53.95 \\
\hline$(\mathrm{C}-37-12) 14 \mathrm{dbc}-1$ & 5,493 & 10-06-1955 & 42 & $\mathrm{D}$ & & 03-23-1941 & 52.49 \\
\hline & & 08-21-1995 & 54.32 & & & $12-02-1941$ & 53.80 \\
\hline & & 10-04-1996 & 57.64 & & & 03-16-1942 & 51.30 \\
\hline & & $11-17-1998$ & 53.60 & & & $12-16-1942$ & 50.25 \\
\hline & & 03-12-1999 & 50.00 & & & $03-22-1943$ & 48.95 \\
\hline & & 11-01-1999 & 59.10 & & & $12-11-1943$ & 51.39 \\
\hline & & $03-13-2000$ & 54.54 & & & $12-07-1944$ & 51.11 \\
\hline & & & & & & 04-02-1945 & 50.95 \\
\hline (C-37-12)14dbd-1 & 5,498 & 07-15-1961 & 60 & $\mathrm{D}$ & & $12-08-1945$ & 51.53 \\
\hline & & 10-04-1973 & 64.51 & & & 03-19-1946 & 49.77 \\
\hline & & 03-21-1974 & 51 & $\mathrm{R}$ & & $12-15-1946$ & 54.23 \\
\hline & & 08-21-1995 & 57.42 & & & 03-24-1947 & 52.29 \\
\hline & & 10-04-1996 & 62.19 & & & $12-08-1947$ & 53.73 \\
\hline & & 03-17-1998 & 57.53 & & & 03-14-1948 & 51.98 \\
\hline & & 11-17-1998 & 59.59 & & & 07-17-1948 & 66.00 \\
\hline & & 03-12-1999 & 56.12 & & & $12-09-1948$ & 55.18 \\
\hline & & 11-01-1999 & 68.87 & & & $12-08-1949$ & 53.87 \\
\hline & & $03-13-2000$ & 60.84 & & & 03-23-1950 & 52.38 \\
\hline & & & & & & $12-07-1950$ & 57.44 \\
\hline (C-37-12)23abd-1 & 5,530 & 03-03-1997 & 90.99 & & & $03-22-1951$ & 54.17 \\
\hline & & 03-17-1998 & 98.22 & & & $12-07-1951$ & 59.16 \\
\hline & & $11-17-1998$ & 95.32 & & & 04-04-1952 & 55.88 \\
\hline & & 03-12-1999 & 91.00 & & & $12-05-1952$ & 59.32 \\
\hline & & $03-13-2000$ & 96.53 & & & 03-14-1953 & 56.14 \\
\hline & & & & & & $12-07-1953$ & 61.82 \\
\hline (C-37-12)23acb-1 & 5,512 & 09-14-1935 & 51.55 & & & 03-19-1955 & 60.20 \\
\hline & & $09-27-1936$ & 51.21 & & & $12-02-1955$ & 67.13 \\
\hline & & $10-24-1937$ & 49.60 & & & 03-18-1956 & 62.75 \\
\hline & & $12-06-1937$ & 49.50 & & & $12-17-1956$ & 58.29 \\
\hline & & 06-08-1938 & 48.96 & & & 03-13-1957 & 55.39 \\
\hline & & 06-18-1938 & 48.86 & & & $10-28-1957$ & 58.64 \\
\hline & & $08-11-1938$ & 48.83 & & & $12-04-1957$ & 68.66 \\
\hline & & $08-29-1938$ & 48.58 & & & 03-18-1958 & 66.19 \\
\hline & & 09-23-1938 & 48.46 & & & $12-22-1958$ & 68.86 \\
\hline & & $11-01-1938$ & 48.20 & & & 04-09-1959 & 62.60 \\
\hline & & $12-01-1938$ & 48.22 & & & $10-21-1959$ & 68.28 \\
\hline & & 01-03-1939 & 48.14 & & & $12-08-1959$ & 66.34 \\
\hline & & 02-03-1939 & 48.13 & & & $03-15-1960$ & 64.25 \\
\hline & & 03-01-1939 & 48.22 & & & $10-28-1960$ & 77.04 \\
\hline
\end{tabular}


Table 2. Water levels in selected wells in Cedar Valley, Iron County, southwestern Utah-Continued

\begin{tabular}{|c|c|c|c|c|c|c|c|}
\hline \multirow{2}{*}{\multicolumn{2}{|c|}{$\begin{array}{cc}\text { Local well number } & \begin{array}{c}\text { Altitude of } \\
\text { land } \\
\text { surface } \\
\text { (feet) }\end{array} \\
\text { (C-37-12)23acb-1-Continued }\end{array}$}} & \multirow{2}{*}{$\begin{array}{c}\text { Date } \\
12-02-1960\end{array}$} & \multicolumn{2}{|l|}{$\begin{array}{l}\text { Water level } \\
\text { (feet) }\end{array}$} & $\begin{array}{c}\text { Altitude of } \\
\text { land } \\
\text { surface } \\
\text { (feet) }\end{array}$ & Date & \multirow[t]{2}{*}{$\begin{array}{l}\text { Water level } \\
\text { (feet) }\end{array}$} \\
\hline & & & 71.70 & & (C-37-12)34abb-1—Continued & $12-09-1948$ & \\
\hline & & $10-10-1962$ & 67.55 & & & 12-08-1949 & 36.51 \\
\hline & & $11-17-1998$ & 72.40 & & & 03-23-1950 & 35.43 \\
\hline & & 03-12-1999 & 69.43 & & & $12-07-1950$ & 40.50 \\
\hline & & $03-13-2000$ & 74.74 & & & 03-22-1951 & 39.11 \\
\hline & & & & & & $12-07-1951$ & 43.78 \\
\hline \multirow[t]{9}{*}{ (C-37-12)28aac-1 } & 5,550 & 09-11-1978 & 99 & D & & 04-04-1952 & 41.71 \\
\hline & & 08-25-1995 & 95.45 & & & $12-05-1952$ & 39.41 \\
\hline & & 10-13-1995 & 97.03 & & & $12-07-1953$ & 44.48 \\
\hline & & $10-04-1996$ & 98.67 & & & 03-23-1954 & 42.84 \\
\hline & & $11-17-1998$ & 99.13 & & & 03-19-1955 & 46.40 \\
\hline & & 03-12-1999 & 98.30 & & & $12-02-1955$ & 52.28 \\
\hline & & 11-01-1999 & 102.67 & & & 03-18-1956 & 49.07 \\
\hline & & 03-13-2000 & 102.03 & & & $12-17-1956$ & 56.49 \\
\hline & & & & & & 03-29-1957 & 52.16 \\
\hline \multirow[t]{36}{*}{ (C-37-12)34abb-1 } & 5,507 & 08-22-1934 & 39.70 & & & $10-28-1957$ & 59.11 \\
\hline & & $10-13-1935$ & 46.93 & & & $12-04-1957$ & 56.57 \\
\hline & & $11-25-1935$ & 46.17 & & & 03-27-1958 & 51.86 \\
\hline & & 01-28-1936 & 45.46 & & & $10-27-1958$ & 52.41 \\
\hline & & 09-27-1936 & 49.15 & & & $12-22-1958$ & 51.57 \\
\hline & & $11-24-1936$ & 47.57 & & & 04-09-1959 & 47.66 \\
\hline & & 03-29-1937 & 45.42 & & & $10-21-1959$ & 58.43 \\
\hline & & 06-07-1937 & 43.00 & & & $12-08-1959$ & 55.49 \\
\hline & & $12-06-1937$ & 43.93 & & & 03-15-1960 & 52.20 \\
\hline & & 02-21-1938 & 42.66 & & & $10-31-1960$ & 60.68 \\
\hline & & 04-17-1938 & 41.90 & & & $12-02-1960$ & 59.86 \\
\hline & & 06-08-1938 & 39.55 & & & 04-04-1961 & 54.11 \\
\hline & & 09-23-1938 & 41.30 & & & $10-16-1961$ & 59.57 \\
\hline & & $11-01-1938$ & 41.23 & & & 03-20-1962 & 53.46 \\
\hline & & $12-01-1938$ & 40.18 & & & $10-17-1962$ & 57.20 \\
\hline & & 01-03-1939 & 39.82 & & & $03-25-1963$ & 51.15 \\
\hline & & 03-01-1939 & 39.77 & & & 03-27-1964 & 54.82 \\
\hline & & 04-04-1939 & 39.87 & & & 11-03-1964 & 59.34 \\
\hline & & 09-11-1939 & 44.65 & & & 03-19-1965 & 54.23 \\
\hline & & $12-09-1939$ & 41.79 & & & $11-05-1965$ & 53.92 \\
\hline & & $03-22-1940$ & 42.02 & & & 03-07-1966 & 51.46 \\
\hline & & 04-13-1940 & 40.70 & & & 03-06-1967 & 50.70 \\
\hline & & $12-02-1940$ & 46.65 & & & $10-06-1967$ & 55.10 \\
\hline & & $03-22-1941$ & 44.84 & & & 03-07-1968 & 48.25 \\
\hline & & $12-02-1941$ & 43.93 & & & 03-17-1969 & 44.46 \\
\hline & & $12-16-1942$ & 38.61 & & & 03-10-1970 & 42.05 \\
\hline & & $03-22-1943$ & 36.86 & & & 03-11-1971 & 45.53 \\
\hline & & $12-11-1943$ & 39.78 & & & 03-09-1972 & 46.92 \\
\hline & & $12-07-1944$ & 39.07 & & & 03-05-1973 & 49.04 \\
\hline & & 04-02-1945 & 37.89 & & & 03-11-1974 & 39.79 \\
\hline & & $12-08-1945$ & 36.78 & & & 11-13-1974 & 47.23 \\
\hline & & 03-19-1946 & 36.05 & & & 03-07-1975 & 42.88 \\
\hline & & $12-15-1946$ & 40.83 & & & 03-01-1976 & 41.17 \\
\hline & & 03-24-1947 & 38.53 & & & 03-01-1977 & 43.91 \\
\hline & & $12-09-1947$ & 39.43 & & & $11-21-1977$ & 52.76 \\
\hline & & 03-14-1948 & 38.59 & & & 03-03-1978 & 49.24 \\
\hline
\end{tabular}


Table 2. Water levels in selected wells in Cedar Valley, Iron County, southwestern Utah-Continued

\begin{tabular}{|c|c|c|c|c|c|c|c|}
\hline Local well number & $\begin{array}{c}\text { Altitude of } \\
\text { land } \\
\text { surface } \\
\text { (feet) }\end{array}$ & Date & $\begin{array}{l}\text { Water level } \\
\text { (feet) }\end{array}$ & Local well number & $\begin{array}{l}\text { Altitude of } \\
\text { land } \\
\text { surface } \\
\text { (feet) }\end{array}$ & Date & $\begin{array}{c}\text { Water level } \\
\text { (feet) }\end{array}$ \\
\hline \multirow{27}{*}{\multicolumn{2}{|c|}{ (C-37-12)34abb-1-Continued }} & 03-16-1978 & 48.35 & \multirow{27}{*}{\multicolumn{2}{|c|}{ (C-38-12)9aab-1-Continued }} & 03-03-1978 & 47.05 \\
\hline & & 03-12-1979 & 40.38 & & & 03-16-1978 & 46.95 \\
\hline & & 03-07-1980 & 32.51 & & & 10-03-1978 & 44.78 \\
\hline & & 03-04-1981 & 27.64 & & & 03-06-1979 & 45.40 \\
\hline & & 03-02-1982 & 32.42 & & & 10-01-1979 & 41.33 \\
\hline & & 03-08-1983 & 31.80 & & & 03-07-1980 & 41.81 \\
\hline & & 03-01-1984 & 25.10 & & & 03-04-1981 & 39.69 \\
\hline & & 03-04-1985 & 27.85 & & & 03-02-1982 & 41.43 \\
\hline & & $10-21-1985$ & 32.40 & & & 03-08-1983 & 42.35 \\
\hline & & 03-03-1986 & 27.38 & & & 03-01-1984 & 40.83 \\
\hline & & 09-29-1986 & 32.68 & & & 03-04-1985 & 41.73 \\
\hline & & 03-08-1989 & 30.97 & & & 03-03-1986 & 43.70 \\
\hline & & 03-05-1990 & 37.35 & & & 03-09-1987 & 43.66 \\
\hline & & 03-01-1991 & 41.63 & & & 03-02-1988 & 43.83 \\
\hline & & 03-10-1992 & 41.41 & & & 03-08-1989 & 43.82 \\
\hline & & 03-08-1993 & 38.70 & & & 03-05-1990 & 44.66 \\
\hline & & 03-09-1994 & 31.20 & & & 03-10-1992 & 48.87 \\
\hline & & 03-09-1995 & 35.33 & & & 03-08-1993 & 48.78 \\
\hline & & 03-08-1996 & 28.85 & & & 03-09-1994 & 50.88 \\
\hline & & 03-03-1997 & 34.49 & & & 03-09-1995 & 49.45 \\
\hline & & $11-25-1997$ & 39.60 & & & 03-08-1996 & 46.65 \\
\hline & & 03-17-1998 & 36.75 & & & 03-03-1997 & 47.56 \\
\hline & & 03-12-1999 & 33.18 & & & $11-25-1997$ & 48.38 \\
\hline & & 12-02-1999 & 41.60 & & & 03-17-1998 & 47.90 \\
\hline & & $03-13-2000$ & 37.75 & & & 03-12-1999 & 46.26 \\
\hline & & 03-09-2001 & 40.38 & & & 11-01-1999 & 47.06 \\
\hline & & & & & & 03-13-2000 & 47.02 \\
\hline \multirow[t]{8}{*}{ (C-38-12)9aab-1 } & \multirow[t]{8}{*}{5,407} & 03-09-1972 & 51.20 & & & 03-09-2001 & 47.09 \\
\hline & & 03-05-1973 & 51.60 & & & & \\
\hline & & 03-11-1974 & 46.91 & \multirow[t]{6}{*}{ (C-38-12)9bba-1 } & \multirow[t]{6}{*}{5,335} & $10-10-1962$ & 26.27 \\
\hline & & 03-05-1975 & 46.96 & & & 10-11-1995 & 17.82 \\
\hline & & 03-01-1976 & 46.53 & & & 10-02-1996 & 19.18 \\
\hline & & $10-07-1976$ & 46.61 & & & 03-17-1998 & 18.55 \\
\hline & & 03-01-1977 & 46.67 & & & 11-01-1999 & 19.95 \\
\hline & & $11-20-1977$ & 47.09 & & & $03-13-2000$ & 17.70 \\
\hline
\end{tabular}


Table 3. Field measurements of $\mathrm{pH}$, specific conductance, and temperature of water from selected wells in Cedar Valley, Iron County, southwestern Utah $[-$, no data available $]$

Local well number: See figure 2 for an explanation of the numbering system used for hydrologic-data sites in Utah. See plate 2 for location of wells with field water-quality measurements.

Specific conductance: $\mu \mathrm{S} / \mathrm{cm}$, microsiemens per centimeter at 25 degrees Celsius.

Water temperature: ${ }^{\circ} \mathrm{C}$, degrees Celsius.

\begin{tabular}{|c|c|c|c|c|}
\hline Local well number & Date & $\begin{array}{c}\mathrm{pH} \\
\text { (standard units) }\end{array}$ & $\begin{array}{l}\text { Specific conductance } \\
(\mu \mathrm{S} / \mathbf{c m})\end{array}$ & $\begin{array}{c}\text { Water } \\
\text { temperature } \\
\left({ }^{\circ} \mathrm{C}\right)\end{array}$ \\
\hline \multirow[t]{3}{*}{ (C-33-10)31ada-1 } & $08-22-1974$ & - & 480 & 14.5 \\
\hline & 07-26-1999 & 7.7 & 720 & - \\
\hline & $06-12-2000$ & 7.8 & 760 & 16.0 \\
\hline \multirow[t]{3}{*}{ (C-33-11)30bca-1 } & $06-25-1974$ & 7.5 & 1,860 & 13.5 \\
\hline & 08-26-1998 & - & 1,730 & 13.5 \\
\hline & $03-13-2000$ & - & 1,740 & 13.5 \\
\hline (C-33-11)31 aad-1 & 07-26-1999 & 8.1 & 1,200 & 15.5 \\
\hline \multirow[t]{3}{*}{ (C-33-11)33caa-1 } & 03-18-1974 & - & 600 & 11.0 \\
\hline & 08-11-1998 & 7.6 & 530 & 13.0 \\
\hline & 03-13-2000 & - & 490 & 12.0 \\
\hline (C-34-10)31caa-1 & 05-23-1974 & 7.9 & 800 & 16.0 \\
\hline (C-34-11) 1daa-1 & 08-21-1974 & - & 500 & 13.5 \\
\hline \multirow[t]{2}{*}{ (C-34-11) 2dad-1 } & 08-26-1998 & - & 490 & 16.0 \\
\hline & 03-13-2000 & - & 480 & 16.0 \\
\hline (C-34-11) 9ccd-1 & 09-10-1974 & - & 800 & 13.5 \\
\hline \multirow[t]{2}{*}{ (C-34-11)14aad-2 } & 08-27-1998 & - & 890 & 16.0 \\
\hline & 07-26-1999 & 8.7 & 930 & 14.0 \\
\hline (C-34-11)20dba-1 & 09-10-1974 & - & 1,180 & 13.0 \\
\hline \multirow[t]{2}{*}{ (C-34-11)21dcd-1 } & 09-10-1974 & - & 730 & 11.5 \\
\hline & 08-10-1998 & 7.8 & 810 & 12.0 \\
\hline & 07-22-1999 & 7.6 & 800 & 15.5 \\
\hline (C-34-11)23bdd-1 & 06-13-1974 & 7.7 & 1,350 & 13.0 \\
\hline$(\mathrm{C}-34-11) 36 \mathrm{dcc}-2$ & 06-13-1977 & 7.5 & 530 & 22.0 \\
\hline \multirow[t]{2}{*}{$(\mathrm{C}-34-11) 36 \mathrm{dcc}-3$} & 08-24-1999 & 7.4 & 590 & 21.0 \\
\hline & $07-25-2000$ & - & 600 & 20.5 \\
\hline$(\mathrm{C}-35-10) 7 \mathrm{abc}-1$ & 08-27-1974 & - & 500 & 15.5 \\
\hline (C-35-10)18abc-1 & $07-19-2000$ & 7.7 & 465 & 15.5 \\
\hline (C-35-10)18bbc-1 & 08-15-1974 & - & 850 & 14.0 \\
\hline \multirow[t]{2}{*}{ (C-35-11) 8ddc-1 } & 08-22-1974 & - & 600 & 13.5 \\
\hline & 09-11-1974 & - & 610 & 12.5 \\
\hline \multirow[t]{10}{*}{ (C-35-11) 9dba-1 } & 10-11-1977 & 7.9 & 730 & 18.0 \\
\hline & 07-19-1979 & 7.7 & 740 & 19.5 \\
\hline & 07-18-1980 & - & 720 & 14.5 \\
\hline & 06-11-1981 & 7.6 & 610 & 15.0 \\
\hline & 08-31-1984 & 7.6 & 620 & 19.0 \\
\hline & 06-14-1985 & - & 700 & 14.0 \\
\hline & 06-27-1986 & 8.1 & 610 & 18.0 \\
\hline & 08-18-1987 & 7.8 & 550 & 14.5 \\
\hline & 06-08-1988 & 7.8 & 560 & 14.0 \\
\hline & 07-11-1989 & 7.8 & 630 & 18.5 \\
\hline
\end{tabular}


Table 3. Field measurements of pH, specific conductance, and temperature of water from selected wells in Cedar Valley, Iron County, southwestern UtahContinued

\begin{tabular}{|c|c|c|c|c|}
\hline Local well number & Date & $\begin{array}{c}\mathrm{pH} \\
\text { (standard units) }\end{array}$ & $\begin{array}{l}\text { Specific conductance } \\
\qquad(\mu \mathrm{S} / \mathbf{c m})\end{array}$ & $\begin{array}{c}\text { Water } \\
\text { temperature } \\
\left({ }^{\circ} \mathrm{C}\right)\end{array}$ \\
\hline \multirow[t]{11}{*}{ (C-35-11) 9dba-1-Continued } & $07-05-1990$ & 7.8 & 640 & 18.0 \\
\hline & 06-04-1991 & 7.9 & 860 & 15.0 \\
\hline & 07-22-1992 & 7.8 & 660 & 14.0 \\
\hline & 07-20-1993 & 7.7 & 670 & 18.5 \\
\hline & 06-22-1994 & 7.7 & 690 & 17.5 \\
\hline & 06-27-1995 & 7.8 & 640 & 14.5 \\
\hline & 07-31-1996 & - & 660 & 17.5 \\
\hline & 05-21-1997 & 8.2 & 630 & 14.5 \\
\hline & 07-10-1998 & 7.2 & 630 & 15.5 \\
\hline & 07-12-1999 & - & 650 & 18.5 \\
\hline & $06-12-2000$ & 7.9 & 640 & 14.5 \\
\hline \multirow[t]{20}{*}{ (C-35-11)11ccc-1 } & 07-19-1979 & 7.7 & 910 & 14.0 \\
\hline & 07-18-1980 & 7.4 & 890 & 14.0 \\
\hline & 05-27-1981 & - & 870 & 13.5 \\
\hline & 07-07-1983 & 6.7 & 940 & 14.0 \\
\hline & 08-31-1984 & - & 860 & 15.5 \\
\hline & 06-10-1985 & - & 930 & 16.0 \\
\hline & 08-18-1987 & 7.6 & 880 & 14.5 \\
\hline & 06-08-1988 & 7.9 & 900 & 14.0 \\
\hline & 07-11-1989 & 7.8 & 910 & 15.0 \\
\hline & 07-05-1990 & 7.8 & 920 & 14.5 \\
\hline & 06-07-1991 & 7.9 & 980 & 15.0 \\
\hline & 08-04-1992 & 7.7 & 930 & 14.0 \\
\hline & 07-20-1993 & 7.6 & 920 & 15.0 \\
\hline & 06-22-1994 & 7.7 & 930 & 15.0 \\
\hline & 06-27-1995 & 7.8 & 910 & 15.5 \\
\hline & 07-31-1996 & - & 910 & 15.0 \\
\hline & 05-21-1997 & 8.0 & 920 & 13.5 \\
\hline & 07-10-1998 & 7.6 & 830 & 15.0 \\
\hline & 07-12-1999 & 7.5 & 920 & 15.0 \\
\hline & $07-25-2000$ & 7.8 & 950 & 15.0 \\
\hline (C-35-11)12add-1 & 07-22-1999 & 7.6 & 455 & 17.5 \\
\hline (C-35-11)12ccc-1 & 08-15-1974 & - & 1,300 & 14.5 \\
\hline \multirow[t]{2}{*}{$(\mathrm{C}-35-11) 12 \mathrm{ccc}-2$} & 08-25-1999 & 7.9 & 760 & 15.5 \\
\hline & $07-19-2000$ & 7.6 & 740 & 15.0 \\
\hline (C-35-11)13ddb-1 & 08-15-1974 & - & 1,200 & 14.5 \\
\hline (C-35-11)14bac-2 & 03-13-2001 & 7.2 & 2,970 & 13.0 \\
\hline \multirow[t]{2}{*}{ (C-35-11)14bca-1 } & 08-25-1999 & 7.2 & 1,810 & - \\
\hline & $07-19-2000$ & 7.4 & 1,920 & - \\
\hline (C-35-11)16aab-1 & 09-01-1999 & 7.4 & 1,070 & 16.0 \\
\hline (C-35-11)17dcd-2 & 03-14-2000 & - & 570 & 13.5 \\
\hline (C-35-11)19dbb-1 & 07-28-1999 & 8.1 & 770 & 17.5 \\
\hline (C-35-11)19dcc-1 & 08-25-1998 & - & 690 & 15.0 \\
\hline \multirow[t]{2}{*}{ (C-35-11)20dda-1 } & 08-24-1998 & - & 700 & 14.5 \\
\hline & $07-26-2000$ & 7.4 & 740 & 13.5 \\
\hline (C-35-11)21abd-1 & 08-14-1998 & - & 830 & 15.0 \\
\hline
\end{tabular}


Table 3. Field measurements of pH, specific conductance, and temperature of water from selected wells in Cedar Valley, Iron County, southwestern UtahContinued

\begin{tabular}{|c|c|c|c|c|}
\hline Local well number & Date & $\begin{array}{c}\mathrm{pH} \\
\text { (standard units) }\end{array}$ & $\begin{array}{c}\text { Specific conductance } \\
(\mu \mathrm{S} / \mathrm{cm})\end{array}$ & $\begin{array}{c}\text { Water } \\
\text { temperature } \\
\left({ }^{\circ} \mathrm{C}\right)\end{array}$ \\
\hline \multirow[t]{2}{*}{$(\mathrm{C}-35-11) 21 \mathrm{dbd}-2$} & 09-09-1974 & - & 880 & 12.5 \\
\hline & 05-21-1997 & 7.3 & 990 & 12.5 \\
\hline (C-35-11)24aab-1 & 08-15-1974 & - & 650 & 15.5 \\
\hline (C-35-11)25bcc-1 & 07-28-1999 & 7.8 & 990 & 15.0 \\
\hline \multirow[t]{5}{*}{ (C-35-11)26acd-1 } & 08-15-1974 & - & 1,100 & 14.5 \\
\hline & 09-13-1974 & - & 1,100 & 14.0 \\
\hline & 08-22-1998 & 7.2 & 980 & 14.5 \\
\hline & 08-24-1999 & 7.2 & 1,000 & 13.5 \\
\hline & $06-22-2000$ & 7.3 & 1,020 & 14.0 \\
\hline \multirow[t]{2}{*}{ (C-35-11)27acc-1 } & 09-09-1974 & - & 1,200 & 11.5 \\
\hline & 08-14-1998 & - & 950 & 11.0 \\
\hline (C-35-11)27bbc-1 & 07-17-1998 & - & 1,040 & 11.0 \\
\hline (C-35-11)27bca-1 & 07-17-1998 & - & 850 & 10.5 \\
\hline \multirow[t]{2}{*}{ (C-35-11)27dbb-1 } & 08-14-1998 & - & 920 & 12.5 \\
\hline & 08-31-1999 & 7.1 & 1,020 & 11.5 \\
\hline \multirow[t]{2}{*}{ (C-35-11)28aac-2 } & 07-17-1998 & - & 1,220 & 14.0 \\
\hline & 08-26-1999 & 7.1 & 1,270 & 11.5 \\
\hline \multirow[t]{3}{*}{ (C-35-11)29add-1 } & $11-07-1973$ & - & 1,650 & 10.5 \\
\hline & 08-27-1999 & 7.5 & 1,610 & 11.5 \\
\hline & 06-12-2000 & 7.5 & 1,640 & 16.0 \\
\hline (C-35-11)29cca-1 & 08-24-1998 & - & 930 & 15.0 \\
\hline \multirow[t]{2}{*}{ (C-35-11)29dbd-2 } & $07-26-2000$ & 6.8 & 2,180 & 11.0 \\
\hline & $07-27-2000$ & 6.9 & 2,160 & 10.5 \\
\hline \multirow[t]{2}{*}{ (C-35-11)30caa-1 } & 08-24-1998 & - & 530 & 16.0 \\
\hline & 08-23-1999 & 7.7 & 540 & 14.0 \\
\hline \multirow[t]{2}{*}{ (C-35-11)31acd-2 } & 08-15-1979 & 7.7 & 630 & 13.0 \\
\hline & 08-25-1998 & - & 880 & 15.0 \\
\hline \multirow[t]{15}{*}{ (C-35-11)31dbd-1 } & $10-11-1977$ & 8.3 & 750 & 13.5 \\
\hline & 07-18-1980 & 7.3 & 640 & 12.5 \\
\hline & 08-04-1983 & 7.7 & 730 & 14.0 \\
\hline & $06-27-1986$ & 7.6 & 620 & 15.0 \\
\hline & 08-18-1987 & 8.1 & 560 & 14.5 \\
\hline & 06-04-1991 & 8.0 & 710 & 14.5 \\
\hline & 08-04-1992 & 8.9 & 650 & 14.0 \\
\hline & 07-19-1993 & 7.8 & 660 & 15.0 \\
\hline & 06-22-1994 & 7.9 & 680 & 14.0 \\
\hline & $06-27-1995$ & 8.0 & 670 & 13.5 \\
\hline & 08-05-1996 & - & 720 & 15.5 \\
\hline & 05-21-1997 & 7.8 & 740 & 14.5 \\
\hline & 07-10-1998 & 7.3 & 740 & 15.0 \\
\hline & 07-13-1999 & 7.8 & 800 & 14.5 \\
\hline & $06-12-2000$ & 8.2 & 870 & 14.5 \\
\hline (C-35-11)32bda-1 & $05-22-1975$ & - & 1,250 & 11.0 \\
\hline (C-35-11)32dba-1 & $05-22-1975$ & - & 1,250 & 11.0 \\
\hline (C-35-11)33aac-1 & 09-24-1963 & 7.2 & 1,610 & - \\
\hline
\end{tabular}


Table 3. Field measurements of pH, specific conductance, and temperature of water from selected wells in Cedar Valley, Iron County, southwestern UtahContinued

\begin{tabular}{|c|c|c|c|c|}
\hline Local well number & Date & $\begin{array}{c}\mathrm{pH} \\
\text { (standard units) }\end{array}$ & $\begin{array}{c}\text { Specific conductance } \\
(\mu \mathrm{S} / \mathbf{c m})\end{array}$ & $\begin{array}{c}\text { Water } \\
\text { temperature } \\
\left({ }^{\circ} \mathrm{C}\right)\end{array}$ \\
\hline \multirow[t]{15}{*}{ (C-35-11)33aac-1-Continued } & $05-22-1974$ & 7.5 & - & 12.5 \\
\hline & 06-19-1975 & 7.2 & 1,400 & 11.0 \\
\hline & 06-17-1976 & 7.2 & 1,400 & 12.0 \\
\hline & 06-13-1977 & 7.2 & 1,350 & 12.5 \\
\hline & 08-01-1978 & 6.9 & 1,440 & 12.0 \\
\hline & 07-21-1981 & 7.0 & 1,010 & 13.0 \\
\hline & $08-22-1985$ & 7.2 & 1,000 & 11.0 \\
\hline & 06-13-1986 & 7.4 & 1,090 & 11.0 \\
\hline & 08-18-1987 & 7.5 & 1,100 & 12.0 \\
\hline & 07-14-1988 & 7.5 & 1,120 & 12.0 \\
\hline & 07-11-1989 & 7.4 & 1,100 & 12.0 \\
\hline & 07-05-1990 & 7.3 & 1,120 & 13.0 \\
\hline & 07-15-1991 & 7.4 & 1,240 & 13.0 \\
\hline & 07-22-1992 & 7.2 & 1,160 & 12.0 \\
\hline & 07-19-1993 & 7.1 & 1,190 & 12.5 \\
\hline \multirow[t]{3}{*}{ (C-35-11)33abd-1 } & 11-09-1973 & - & 1,600 & 11.0 \\
\hline & 07-17-1998 & 7.3 & 1,200 & 13.0 \\
\hline & 08-25-1999 & 7.1 & 1,270 & 11.5 \\
\hline \multirow[t]{2}{*}{ (C-35-11)33bad-1 } & $11-09-1973$ & - & 1,800 & 11.0 \\
\hline & 07-17-1998 & 7.3 & 1,700 & 13.0 \\
\hline (C-35-11)33bcc-1 & 11-08-1973 & - & 1,180 & 10.0 \\
\hline \multirow[t]{4}{*}{ (C-35-11)33ccd-1 } & 05-21-1997 & 7.6 & 1,170 & 12.5 \\
\hline & 05-21-1998 & - & 1,100 & 13.5 \\
\hline & 07-15-1998 & - & 1,190 & 14.5 \\
\hline & 08-25-1999 & 7.2 & 1,290 & 12.5 \\
\hline \multirow[t]{2}{*}{ (C-35-11)34dbb-1 } & 07-15-1998 & 7.0 & 1,130 & 15.5 \\
\hline & 09-01-1999 & 7.3 & 1,190 & 14.0 \\
\hline \multirow[t]{2}{*}{ (C-35-12)25ddd-1 } & $11-07-1973$ & - & 3,000 & 12.0 \\
\hline & 08-25-1998 & - & 1,330 & 13.0 \\
\hline \multirow[t]{2}{*}{ (C-35-12)26bca-1 } & 05-18-1998 & 7.4 & 1,020 & 15.5 \\
\hline & 07-21-1999 & 7.2 & 1,050 & 12.5 \\
\hline (C-35-12)27bcc-1 & $10-10-1973$ & - & 860 & 14.5 \\
\hline (C-35-12)36аaа-1 & 08-25-1998 & - & 690 & 14.5 \\
\hline \multirow[t]{2}{*}{$(\mathrm{C}-35-12) 36$ caa-1 } & 05-19-1997 & 7.7 & 630 & 16.0 \\
\hline & 08-23-1999 & 7.5 & 650 & 16.5 \\
\hline (C-35-12)36caa-2 & 03-14-2001 & 7.1 & 3,580 & 12.0 \\
\hline \multirow[t]{3}{*}{ (C-35-12)36ddd-1 } & 05-19-1997 & 7.8 & 530 & 13.5 \\
\hline & 05-20-1998 & 7.4 & 530 & 16.0 \\
\hline & 08-26-1999 & 7.9 & 540 & 14.0 \\
\hline (C-36-11) 5abb-1 & 05-21-1997 & 7.1 & 2,000 & 11.5 \\
\hline \multirow[t]{2}{*}{ (C-36-11) 5aca-1 } & 05-23-1974 & 7.6 & 1,300 & 11.0 \\
\hline & 08-26-1999 & 7.0 & 1,780 & 11.5 \\
\hline (C-36-11) 5acc-1 & 07-15-1998 & - & 1,960 & 13.0 \\
\hline (C-36-11) 5cac-1 & 07-15-1998 & - & 1,290 & 16.0 \\
\hline \multirow[t]{2}{*}{ (C-36-11) 5cba-1 } & $11-18-1974$ & - & 1,500 & 12.5 \\
\hline & 08-27-1997 & 7.6 & 780 & 12.5 \\
\hline
\end{tabular}


Table 3. Field measurements of pH, specific conductance, and temperature of water from selected wells in Cedar Valley, Iron County, southwestern UtahContinued

\begin{tabular}{|c|c|c|c|c|}
\hline Local well number & Date & $\begin{array}{c}\mathrm{pH} \\
\text { (standard units) }\end{array}$ & $\begin{array}{c}\text { Specific conductance } \\
(\mu \mathrm{S} / \mathrm{cm})\end{array}$ & $\begin{array}{c}\text { Water } \\
\text { temperature } \\
\left({ }^{\circ} \mathrm{C}\right)\end{array}$ \\
\hline (C-36-11) 5cba-1-Continued & $07-15-1998$ & - & 1,300 & 16.0 \\
\hline \multirow[t]{2}{*}{ (C-36-11) 5dab-1 } & 07-15-1998 & 7.2 & 1,100 & 14.5 \\
\hline & 09-01-1999 & 7.3 & 1,090 & 12.5 \\
\hline (C-36-11) 7aaa-2 & 09-01-1999 & 7.2 & 1,410 & 13.5 \\
\hline \multirow[t]{2}{*}{ (C-36-11) 7aba-1 } & $11-08-1973$ & - & 1,180 & 11.5 \\
\hline & 07-15-1998 & - & 1,240 & 15.0 \\
\hline (C-36-11) 7cab-1 & 08-23-1999 & 7.8 & 1,060 & 15.5 \\
\hline (C-36-11) 8aab-1 & $10-10-1974$ & - & 920 & 14.0 \\
\hline$(\mathrm{C}-36-11) 8 \mathrm{cbb}-1$ & 05-15-1998 & 7.1 & 2,190 & 13.5 \\
\hline \multirow[t]{4}{*}{ (C-36-11)11bac-1 } & 09-27-1974 & - & 2,200 & 14.0 \\
\hline & 07-15-1998 & 7.0 & 2,260 & 15.0 \\
\hline & 08-26-1999 & 7.7 & 2,220 & 14.5 \\
\hline & $07-25-2000$ & 7.4 & 2,190 & 16.5 \\
\hline (C-36-11)18baa-1 & 05-15-1998 & 7.3 & 1,290 & 15.0 \\
\hline${ }^{1}(\mathrm{C}-36-11) 18 \mathrm{bca}-2$ & $11-14-1973$ & - & 1,100 & 10.5 \\
\hline \multirow[t]{2}{*}{ (C-36-11)18bdd-1 } & 05-15-1998 & 7.1 & 1,660 & 15.0 \\
\hline & 07-22-1999 & 7.5 & 1,440 & 16.5 \\
\hline$(\mathrm{C}-36-11) 31 \mathrm{abc}-1$ & $07-28-2000$ & 7.5 & 1,140 & - \\
\hline (C-36-12) 2dbc-1 & 07-21-1999 & 7.5 & 600 & 19.5 \\
\hline (C-36-12) 3aad-2 & 07-21-1999 & - & 530 & 16.0 \\
\hline \multirow[t]{2}{*}{ (C-36-12) 9aac-1 } & 05-19-1998 & 7.6 & 540 & 17.5 \\
\hline & 07-21-1999 & 7.6 & 540 & - \\
\hline \multirow[t]{23}{*}{$(\mathrm{C}-36-12) 12 \mathrm{dba}-1$} & 09-05-1961 & 7.6 & 710 & - \\
\hline & 07-20-1966 & - & 620 & 13.5 \\
\hline & 09-10-1969 & 7.7 & 550 & 13.0 \\
\hline & $08-28-1970$ & - & 570 & 13.5 \\
\hline & $10-02-1973$ & - & 580 & 13.5 \\
\hline & $05-22-1974$ & 8.0 & 600 & 14.0 \\
\hline & 07-01-1975 & - & 580 & 13.5 \\
\hline & 06-17-1976 & - & 560 & 14.0 \\
\hline & 07-31-1978 & - & 560 & 13.5 \\
\hline & 06-11-1981 & 7.7 & 540 & 13.5 \\
\hline & 06-28-1982 & 8.1 & 560 & 16.0 \\
\hline & 06-24-1986 & 7.4 & 590 & 15.0 \\
\hline & 08-18-1987 & 7.5 & 600 & 15.5 \\
\hline & 07-05-1990 & 7.8 & 570 & 15.5 \\
\hline & 06-07-1991 & 7.8 & 590 & 15.5 \\
\hline & $07-20-1993$ & 7.8 & 580 & 15.0 \\
\hline & 08-23-1994 & 7.8 & 580 & 14.0 \\
\hline & 07-12-1995 & 7.9 & 580 & 15.5 \\
\hline & 08-05-1996 & - & 570 & 14.5 \\
\hline & 05-20-1997 & 7.8 & 590 & 14.5 \\
\hline & 07-13-1998 & 7.8 & 580 & 15.0 \\
\hline & 08-23-1999 & 7.7 & 550 & 14.5 \\
\hline & 06-13-2000 & 7.7 & 590 & 16.0 \\
\hline
\end{tabular}


Table 3. Field measurements of pH, specific conductance, and temperature of water from selected wells in Cedar Valley, Iron County, southwestern UtahContinued

\begin{tabular}{|c|c|c|c|c|}
\hline Local well number & Date & $\begin{array}{c}\mathrm{pH} \\
\text { (standard units) }\end{array}$ & $\begin{array}{l}\text { Specific conductance } \\
(\mu \mathrm{S} / \mathbf{c m})\end{array}$ & $\begin{array}{c}\text { Water } \\
\text { temperature } \\
\left({ }^{\circ} \mathrm{C}\right)\end{array}$ \\
\hline \multirow[t]{3}{*}{ (C-36-12)20acc-1 } & 09-21-1967 & 7.4 & 415 & 14.0 \\
\hline & 06-03-1969 & - & 395 & 14.0 \\
\hline & 09-05-1974 & - & 490 & 14.0 \\
\hline \multirow[t]{10}{*}{ (C-36-12)21cbb-1 } & $10-03-1973$ & - & 390 & 13.0 \\
\hline & $05-22-1974$ & 8.2 & 350 & 12.5 \\
\hline & 06-17-1976 & - & 340 & 16.0 \\
\hline & 06-13-1977 & - & 340 & 16.0 \\
\hline & 06-06-1978 & - & 340 & 14.0 \\
\hline & 07-19-1979 & 8.0 & 350 & 14.5 \\
\hline & 07-18-1980 & 7.7 & 335 & 14.0 \\
\hline & $05-27-1981$ & 7.9 & 320 & 14.0 \\
\hline & 06-30-1982 & 8.0 & 345 & 14.0 \\
\hline & 06-08-1988 & 8.0 & 340 & 14.0 \\
\hline (C-36-12)25bdd-1 & $05-22-1974$ & 7.8 & 750 & 13.5 \\
\hline$(\mathrm{C}-36-12) 32 \mathrm{dcc}-1$ & $05-22-1974$ & - & 260 & 13.0 \\
\hline \multirow[t]{3}{*}{ (C-36-12)36adb-1 } & 09-06-1974 & - & 900 & 14.0 \\
\hline & 07-22-1999 & 7.2 & 860 & - \\
\hline & $07-25-2000$ & 7.1 & 880 & 14.5 \\
\hline \multirow[t]{2}{*}{ (C-37-12) 5acc-2 } & 07-13-1999 & 7.6 & 270 & 15.0 \\
\hline & 08-23-1999 & 7.4 & 265 & 15.5 \\
\hline \multirow[t]{14}{*}{ (C-37-12) 9acc-1 } & 09-09-1974 & - & 340 & 15.5 \\
\hline & 09-11-1974 & - & 360 & 16.0 \\
\hline & 08-04-1983 & 7.5 & 350 & 16.0 \\
\hline & 08-31-1984 & - & 335 & 16.5 \\
\hline & 06-10-1985 & - & 375 & 15.5 \\
\hline & 07-22-1992 & - & 355 & 16.5 \\
\hline & 08-02-1993 & 7.5 & 365 & 16.5 \\
\hline & 06-23-1994 & 7.7 & 365 & 16.5 \\
\hline & 06-28-1995 & 8.0 & 370 & 16.0 \\
\hline & 08-05-1996 & - & 370 & 17.0 \\
\hline & 05-20-1997 & 7.6 & 365 & 16.0 \\
\hline & 07-13-1998 & 7.0 & 375 & 16.0 \\
\hline & 07-13-1999 & 7.9 & 375 & 16.5 \\
\hline & $06-13-2000$ & 7.9 & 380 & 18.0 \\
\hline (C-37-12)11aaa-1 & 06-14-1974 & 7.8 & 570 & 21.0 \\
\hline (C-37-12)11dac-1 & 08-23-1999 & 7.6 & 640 & 18.5 \\
\hline \multirow[t]{2}{*}{ (C-37-12)14abc-1 } & 07-24-1974 & - & 650 & 16.5 \\
\hline & 05-20-1997 & 8.1 & 680 & 17.5 \\
\hline (C-37-12)14dbc-1 & 07-24-1974 & - & 680 & 14.5 \\
\hline \multirow[t]{6}{*}{ (C-37-12)23abd-1 } & 06-04-1991 & 7.9 & 650 & 16.5 \\
\hline & 07-22-1992 & - & 600 & 19.0 \\
\hline & 07-20-1993 & - & 600 & 17.5 \\
\hline & 06-23-1994 & 7.7 & 680 & 16.5 \\
\hline & 08-21-1995 & - & 670 & 15.5 \\
\hline & 07-31-1996 & - & 640 & 16.5 \\
\hline
\end{tabular}


Table 3. Field measurements of $\mathrm{pH}$, specific conductance, and temperature of water from selected wells in Cedar Valley, Iron County, southwestern UtahContinued

\begin{tabular}{|c|c|c|c|c|}
\hline Local well number & Date & $\begin{array}{c}\mathrm{pH} \\
\text { (standard units) }\end{array}$ & $\begin{array}{c}\text { Specific conductance } \\
(\mu \mathrm{S} / \mathrm{cm})\end{array}$ & $\begin{array}{c}\text { Water } \\
\text { temperature } \\
\left({ }^{\circ} \mathrm{C}\right)\end{array}$ \\
\hline \multirow[t]{5}{*}{ (C-37-12)23abd-1-Continued } & $05-20-1997$ & 7.6 & 630 & 16.0 \\
\hline & 07-13-1998 & 7.2 & 650 & 18.5 \\
\hline & 07-13-1999 & 7.4 & 640 & 17.5 \\
\hline & 08-21-1999 & - & 670 & 15.5 \\
\hline & $07-25-2000$ & 7.5 & 650 & 17.0 \\
\hline \multirow[t]{20}{*}{ (C-37-12)23acb-1 } & $05-25-1966$ & - & 550 & 13.5 \\
\hline & $07-25-1968$ & - & 690 & 14.5 \\
\hline & 09-10-1969 & - & 550 & - \\
\hline & 05-04-1970 & - & 820 & 11.0 \\
\hline & 07-02-1971 & - & 620 & 14.0 \\
\hline & $10-03-1973$ & - & 650 & 13.5 \\
\hline & 05-22-1974 & 7.8 & 600 & 13.5 \\
\hline & 09-02-1976 & - & 620 & 15.0 \\
\hline & $06-23-1977$ & - & 700 & 14.0 \\
\hline & 06-06-1978 & - & 780 & 13.5 \\
\hline & 07-19-1979 & 7.7 & 780 & 14.5 \\
\hline & 07-18-1980 & - & 780 & 14.5 \\
\hline & $05-28-1981$ & 7.7 & 780 & 13.5 \\
\hline & 08-24-1983 & - & 740 & - \\
\hline & 08-31-1984 & 7.7 & 760 & 14.5 \\
\hline & 06-10-1985 & - & 840 & 15.0 \\
\hline & 07-19-1990 & 7.7 & 810 & 15.5 \\
\hline & 08-22-1995 & - & 940 & 15.5 \\
\hline & 05-20-1997 & - & 1,020 & 14.0 \\
\hline & 07-13-1998 & 7.1 & 1,050 & 17.5 \\
\hline \multirow[t]{20}{*}{ (C-37-12)34abb-1 } & 09-05-1961 & 7.8 & 690 & 11.5 \\
\hline & 09-01-1964 & 7.7 & 780 & 11.5 \\
\hline & $05-25-1966$ & 7.9 & 900 & 11.0 \\
\hline & 09-01-1967 & 7.7 & 780 & - \\
\hline & $07-12-1968$ & 7.6 & 830 & 12.0 \\
\hline & 08-29-1969 & 8.2 & 710 & 11.0 \\
\hline & 05-04-1970 & - & 840 & 10.5 \\
\hline & 07-02-1971 & - & 880 & 11.0 \\
\hline & 05-12-1972 & 7.2 & 890 & 12.0 \\
\hline & $10-03-1973$ & - & 890 & 12.0 \\
\hline & $05-22-1974$ & 7.6 & 800 & 11.5 \\
\hline & 09-02-1976 & - & 800 & 12.5 \\
\hline & $06-23-1977$ & - & 810 & 12.0 \\
\hline & $07-24-1978$ & - & 940 & 11.5 \\
\hline & 08-15-1979 & 7.0 & 910 & 12.0 \\
\hline & 07-18-1980 & - & 950 & 10.5 \\
\hline & $07-21-1981$ & 7.2 & 920 & 13.0 \\
\hline & $07-30-1982$ & 7.7 & 910 & 11.0 \\
\hline & 08-24-1983 & - & 930 & 11.0 \\
\hline & 08-31-1984 & 7.6 & 810 & 11.0 \\
\hline
\end{tabular}


Table 3. Field measurements of pH, specific conductance, and temperature of water from selected wells in Cedar Valley, Iron County, southwestern UtahContinued

\begin{tabular}{|c|c|c|c|c|}
\hline Local well number & Date & $\begin{array}{c}\mathrm{pH} \\
\text { (standard units) }\end{array}$ & $\begin{array}{c}\text { Specific conductance } \\
(\mu \mathrm{S} / \mathrm{cm})\end{array}$ & $\begin{array}{c}\text { Water } \\
\text { temperature } \\
\left({ }^{\circ} \mathrm{C}\right)\end{array}$ \\
\hline \multirow[t]{16}{*}{ (C-37-12)34abb-1-Continued } & $06-27-1986$ & - & 870 & 16.0 \\
\hline & 08-18-1987 & 7.1 & 870 & 11.0 \\
\hline & 07-15-1988 & - & 810 & 12.0 \\
\hline & 07-11-1989 & 7.2 & 840 & 12.0 \\
\hline & 07-05-1990 & 7.2 & 870 & 12.0 \\
\hline & 06-04-1991 & 7.3 & 790 & 11.5 \\
\hline & 07-22-1992 & 7.0 & 860 & 12.0 \\
\hline & 07-20-1993 & 7.1 & 860 & 12.0 \\
\hline & 06-23-1994 & 7.2 & 840 & 12.0 \\
\hline & 08-16-1995 & 7.2 & 840 & 11.0 \\
\hline & 08-22-1995 & - & 870 & 11.0 \\
\hline & 07-31-1996 & - & 800 & 13.5 \\
\hline & 07-01-1997 & 7.2 & 780 & 12.5 \\
\hline & 07-13-1998 & 7.0 & 800 & 13.0 \\
\hline & 07-13-1999 & 7.0 & 790 & 11.0 \\
\hline & 06-13-2000 & 7.1 & 810 & 11.0 \\
\hline
\end{tabular}

${ }^{1}$ Previously reported for (C-36-11)18bca-1 in Bjorklund and others (1977). 
Table 4. Physical properties and results of chemical analyses of water from selected wells and surface-water sites, Cedar Valley,

[mg/L, milligrams per liter; $\mu \mathrm{g} / \mathrm{L}$, micrograms per liter; —, no data available; $<$, less than; E, estimated]

Local well number: See figure 2 for an explanation of the numbering system used for hydrologic-data sites in Utah. Sites are shown on plate 2 . $\mathrm{pH}$ : Determined in the field except where noted by L, laboratory value.

Specific conductance: $\mu \mathrm{S} / \mathrm{cm}$, microsiemens per centimeter at 25 degrees Celsius. Measured in the field.

Water temperature: ${ }^{\circ} \mathrm{C}$, degrees Celsius. Measured in the field.

Alkalinity: Measured in the field except where noted by L, laboratory value.

\begin{tabular}{|c|c|c|c|c|c|c|c|c|c|c|c|c|}
\hline $\begin{array}{l}\text { Local well } \\
\text { number }\end{array}$ & Date & $\begin{array}{l}\text { pH, water, } \\
\text { whole, } \\
\text { field } \\
\text { (standard } \\
\text { units) }\end{array}$ & $\begin{array}{c}\text { Specific } \\
\text { conduc- } \\
\text { tance } \\
(\mu \mathrm{S} / \mathrm{cm})\end{array}$ & $\begin{array}{c}\text { Water } \\
\text { tempera-ture, } \\
\left({ }^{\circ} \mathrm{C}\right)\end{array}$ & $\begin{array}{c}\text { Hardness, } \\
\text { total } \\
(\mathrm{mg} / \mathrm{L} \text { as } \\
\left.\mathrm{CaCO}_{3}\right)\end{array}$ & $\begin{array}{c}\text { Calcium, } \\
\text { dissolved } \\
\text { (mg/L as Ca) }\end{array}$ & $\begin{array}{l}\text { Magne-sium, } \\
\text { dissolved } \\
(\mathrm{mg} / \mathrm{L} \text { as } \mathrm{Mg})\end{array}$ & $\begin{array}{l}\text { Potas- } \\
\text { sium, } \\
\text { dissolved } \\
\text { (mg/L } \\
\text { as K) }\end{array}$ & $\begin{array}{c}\text { Sodium, } \\
\text { dissolved } \\
\text { (mg/L as } \mathrm{Na})\end{array}$ & \multicolumn{2}{|c|}{$\begin{array}{l}\text { Alkalinity, } \\
\text { water, } \\
\text { dissolved } \\
(\mathrm{mg} / \mathrm{L} \text { as } \\
\left.\mathrm{CaCO}_{3}\right)\end{array}$} & $\begin{array}{c}\text { Bicarbo-nate, } \\
\text { water, } \\
\text { dissolved } \\
\text { (mg/L as } \\
\left.\mathrm{HCO}_{3}\right)\end{array}$ \\
\hline \multicolumn{13}{|c|}{ Wells } \\
\hline \multirow[t]{2}{*}{ (C-33-10)31ada-1 } & 07-26-1999 & 7.7 & 720 & - & 230 & 52 & 25 & 6.1 & 52 & 130 & & 159 \\
\hline & $06-12-2000$ & 7.8 & 760 & 16.0 & 230 & 53 & 25 & 7.1 & 54 & 133 & & 163 \\
\hline (C-33-11)30bca-1 & $06-25-1974$ & 7.5 & 1,860 & 13.5 & 660 & 120 & 87 & 12 & 170 & 278 & & - \\
\hline (C-33-11)31aad-1 & 07-26-1999 & 8.1 & 1,200 & 15.5 & 190 & 25 & 31 & 7.7 & 180 & 197 & & 241 \\
\hline (C-34-11)14aad-2 & 07-26-1999 & 8.7 & 930 & 14.0 & 290 & 44 & 43 & 6.8 & 80 & 212 & & 259 \\
\hline (C-34-11)21dcd-1 & 07-22-1999 & 7.6 & 800 & 15.5 & 320 & 56 & 43 & 4.2 & 49 & 146 & & 178 \\
\hline (C-34-11)36dcc-3 & 08-24-1999 & 7.4 & 590 & 21.0 & 240 & 46 & 30 & 4.8 & 31 & 187 & & 228 \\
\hline (C-35-10) 18abc-1 & $07-19-2000$ & 7.7 & 465 & 15.5 & 200 & 40 & 23 & 4.1 & 17 & 186 & & 227 \\
\hline \multirow[t]{10}{*}{ (C-35-11) 9dba-1 } & $10-11-1977$ & 7.9 & 730 & 18.0 & - & - & - & - & - & - & & - \\
\hline & 07-19-1979 & 7.7 & 740 & 19.5 & 380 & 74 & 47 & 2.8 & 18 & 160 & & - \\
\hline & 06-11-1981 & 7.6 & 610 & 15.0 & 310 & 61 & 38 & 2.6 & 18 & 150 & $\mathrm{~L}$ & - \\
\hline & 08-31-1984 & 7.6 & 620 & 19.0 & 300 & 60 & 37 & 2.9 & 15 & 158 & $\mathrm{~L}$ & - \\
\hline & 06-27-1986 & 8.1 & 610 & 18.0 & 300 & 60 & 37 & 2.6 & 16 & 157 & $\mathrm{~L}$ & - \\
\hline & 07-11-1989 & 7.8 & 630 & 18.5 & 300 & 61 & 37 & 3.2 & 17 & 158 & $\mathrm{~L}$ & - \\
\hline & 06-04-1991 & 7.9 & 860 & 15.0 & 370 & 76 & 44 & 2.2 & 12 & 166 & $\mathrm{~L}$ & - \\
\hline & 07-22-1992 & 7.8 & 660 & 14.0 & 320 & 66 & 38 & 2.2 & 15 & 153 & $\mathrm{~L}$ & - \\
\hline & 06-27-1995 & 7.8 & 640 & 14.5 & 290 & 59 & 35 & 2.4 & 15 & 154 & $\mathrm{~L}$ & - \\
\hline & 07-10-1998 & 7.2 & 630 & 15.5 & 320 & 62 & 40 & 2.2 & 14 & - & & - \\
\hline \multirow[t]{9}{*}{$(\mathrm{C}-35-11) 11 \mathrm{ccc}-1$} & 07-19-1979 & 7.7 & 910 & 14.0 & 440 & 81 & 57 & 4.6 & 29 & 160 & & - \\
\hline & 07-18-1980 & 7.4 & 890 & 14.0 & 410 & 77 & 52 & 4.6 & 28 & 170 & & - \\
\hline & $07-07-1983$ & 6.7 & 940 & 14.0 & 410 & 76 & 54 & 4.2 & 29 & 174 & $\mathrm{~L}$ & - \\
\hline & 08-18-1987 & 7.6 & 880 & 14.5 & 420 & 80 & 54 & 8.8 & 29 & 170 & $\mathrm{~L}$ & - \\
\hline & 07-05-1990 & 7.8 & 920 & 14.5 & 420 & 80 & 53 & 4.7 & 29 & 166 & $\mathrm{~L}$ & - \\
\hline & 07-20-1993 & 7.6 & 920 & 15.0 & 420 & 81 & 53 & 4.5 & 28 & 166 & $\mathrm{~L}$ & - \\
\hline & 07-31-1996 & $7.5 \mathrm{~L}$ & 910 & 15.0 & 430 & 83 & 55 & 4.6 & 29 & 167 & $\mathrm{~L}$ & - \\
\hline & 07-12-1999 & 7.5 & 920 & 15.0 & - & - & - & - & - & - & & - \\
\hline & $07-25-2000$ & 7.8 & 950 & 15.0 & 430 & 83 & 55 & 4.3 & 29 & 161 & & 197 \\
\hline (C-35-11)12add-1 & 07-22-1999 & 7.6 & 455 & 17.5 & 210 & 44 & 23 & 5.3 & 17 & 191 & & 233 \\
\hline \multirow[t]{2}{*}{$(\mathrm{C}-35-11) 12 \mathrm{ccc}-2$} & 08-25-1999 & 7.9 & 760 & 15.5 & 340 & 65 & 42 & 5.5 & 21 & 166 & & 203 \\
\hline & $07-19-2000$ & 7.6 & 740 & 15.0 & 300 & 58 & 37 & 5.4 & 18 & 167 & & 206 \\
\hline (C-35-11)14bac-2 & 03-31-2001 & 7.2 & 2,970 & 13.0 & 1,800 & 320 & 244 & 4.1 & 99 & 221 & & 270 \\
\hline \multirow[t]{2}{*}{ (C-35-11)14bca-1 } & 08-25-1999 & 7.2 & 1,810 & - & 900 & 180 & 107 & 4.9 & 67 & 209 & & 255 \\
\hline & $07-19-2000$ & 7.4 & 1,920 & - & 860 & 180 & 102 & 5.0 & 66 & 210 & & 256 \\
\hline (C-35-11)16aab-1 & 09-01-1999 & 7.4 & 1,070 & 16.0 & 590 & 120 & 69 & 2.8 & 18 & 266 & & 325 \\
\hline (C-35-11)19dbb-1 & 07-28-1999 & 8.1 & 770 & 17.5 & 330 & 63 & 43 & 3.1 & 30 & 111 & & 135 \\
\hline (C-35-11)25bcc-1 & 07-28-1999 & 7.8 & 990 & 15.0 & 330 & 73 & 35 & 4.2 & 63 & 152 & & 185 \\
\hline \multirow[t]{3}{*}{ (C-35-11)26acd-1 } & 09-13-1974 & - & 1,100 & 14.0 & 490 & 93 & 62 & 5.8 & 40 & 249 & & - \\
\hline & 08-24-1999 & 7.2 & 1,000 & 13.5 & 480 & 99 & 57 & 4.5 & 34 & 307 & & 375 \\
\hline & $06-22-2000$ & 7.3 & 1,020 & 14.0 & 460 & 92 & 56 & 4.2 & 34 & 313 & & 382 \\
\hline (C-35-11)27dbb-1 & 08-31-1999 & 7.1 & 1,020 & 11.5 & 530 & 120 & 54 & 2.7 & 17 & 423 & & 516 \\
\hline (C-35-11)28aac-2 & 08-26-1999 & 7.1 & 1,270 & 11.5 & 690 & 150 & 77 & 3.4 & 21 & 349 & & 426 \\
\hline
\end{tabular}


Iron County, southwestern Utah

\begin{tabular}{|c|c|c|c|c|c|c|c|c|c|c|c|c|}
\hline $\begin{array}{l}\text { Local well } \\
\text { number }\end{array}$ & $\begin{array}{c}\text { Chloride, } \\
\text { dis- } \\
\text { solved } \\
\text { (mg/L as } \mathrm{Cl} \text { ) }\end{array}$ & $\begin{array}{l}\text { Fluoride, dis- } \\
\text { solved } \\
\text { (mg/L } \\
\text { as F) }\end{array}$ & $\begin{array}{c}\text { Silica, } \\
\text { dissolved } \\
\text { (mg// } \\
\left.\text { as } \mathrm{SiO}_{2}\right)\end{array}$ & $\begin{array}{c}\text { Sulfate, } \\
\text { dissolved } \\
(\mathrm{mg} / \mathrm{L} \\
\left.\text { as } \mathrm{SO}_{4}\right)\end{array}$ & $\begin{array}{c}\text { Nitrogen, } \\
\text { ammonia + } \\
\text { organic, } \\
\text { dissolved } \\
\text { (mg/L } \\
\text { as N) }\end{array}$ & $\begin{array}{l}\text { Nitrogen, } \\
\mathrm{NO}_{2}+\mathrm{NO}_{3}, \\
\text { dissolved } \\
\text { (mg/L } \\
\text { as N) }\end{array}$ & $\begin{array}{l}\text { Phos-phorus, } \\
\text { dissolved } \\
\text { (mg/L } \\
\text { as P) }\end{array}$ & $\begin{array}{l}\text { Solids, sum } \\
\text { of consti- } \\
\text { tuents, } \\
\text { dissolved } \\
\text { (mg/L) }\end{array}$ & $\begin{array}{c}\text { Boron, } \\
\text { dis- } \\
\text { solved } \\
(\mu \mathrm{g} / \mathrm{L} \\
\text { as B) }\end{array}$ & $\begin{array}{c}\text { Bromide, } \\
\text { dis- } \\
\text { solved } \\
\text { (mg/L } \\
\text { as Br) }\end{array}$ & $\begin{array}{l}\text { Iron, } \\
\text { dis- } \\
\text { solved } \\
(\mu \mathrm{g} / \mathrm{L} \\
\text { as Fe) }\end{array}$ & $\begin{array}{c}\text { Manga- } \\
\text { nese, } \\
\text { dis- } \\
\text { solved } \\
(\mu \mathrm{g} / \mathrm{L} \\
\text { as } \mathrm{Mn})\end{array}$ \\
\hline \multicolumn{13}{|c|}{ Wells } \\
\hline \multirow[t]{2}{*}{ (C-33-10)31 ada-1 } & 93 & .41 & 41 & 53 & .34 & - & - & 401 & 112 & .37 & $<10$ & $<3.0$ \\
\hline & 92 & .37 & 45 & 53 & .23 & 13.3 & $<.050$ & 468 & 102 & .36 & $<10$ & $<2.2$ \\
\hline (C-33-11)30bca-1 & 130 & .70 & 43 & 520 & - & .030 & - & 1,250 & 340 & - & 80 & 480 \\
\hline (C-33-11)31 aad-1 & 91 & 1.1 & 61 & 280 & - & $<.050$ & .051 & 795 & 319 & .031 & 130 & 20 \\
\hline (C-34-11)14aad-2 & 100 & .55 & 37 & 120 & - & .887 & $.034 \mathrm{E}$ & 567 & 130 & .18 & $<10$ & $<3.0$ \\
\hline (C-34-11)21dcd-1 & 16 & .38 & 23 & 250 & - & .148 & $<.050$ & 525 & 126 & .050 & 22 & 2.9 \\
\hline (C-34-11)36dcc-3 & 37 & .40 & 41 & 67 & .10 & .521 & - & 369 & 62 & .057 & $<10$ & $<3.0$ \\
\hline (C-35-10)18abc-1 & 14 & .39 & 42 & 28 & - & .928 & $.036 \quad \mathrm{E}$ & 285 & 62 & .052 & $<10$ & $<2.2$ \\
\hline \multirow[t]{10}{*}{ (C-35-11) 9dba-1 } & - & - & - & - & - & 1.50 & - & - & - & - & - & - \\
\hline & 11 & .40 & 22 & 230 & - & .690 & - & 505 & 60 & - & - & - \\
\hline & 8.7 & .30 & 22 & 180 & - & .420 & .030 & 423 & 40 & - & $<10$ & 2.0 \\
\hline & 8.2 & .40 & 22 & 170 & - & .710 & $<.010$ & 413 & 70 & - & 11 & 1.0 \\
\hline & 8.4 & .30 & 21 & 170 & - & .610 & .010 & 412 & 50 & - & 37 & 3.0 \\
\hline & 8.7 & .30 & 21 & 180 & - & .670 & $<.010$ & 426 & 50 & - & $<3.0$ & 1.0 \\
\hline & 11 & .30 & 20 & 210 & - & - & - & 475 & 40 & - & 26 & 6.0 \\
\hline & 11 & .30 & 20 & 180 & - & .880 & $<.010$ & 428 & 50 & - & 9.0 & $<1.0$ \\
\hline & 7.8 & .30 & 21 & 160 & - & .620 & .050 & 396 & 40 & - & 7.0 & 2.0 \\
\hline & 9.0 & .29 & 23 & 170 & - & .980 & $<.010$ & - & 48 & - & $<10$ & $<4.0$ \\
\hline \multirow[t]{9}{*}{ (C-35-11)11 ccc-1 } & 23 & .30 & 34 & 250 & - & 7.70 & .030 & 609 & 70 & - & - & - \\
\hline & 26 & .30 & 33 & 240 & - & 8.20 & .020 & 600 & 90 & - & $<10$ & $<3.0$ \\
\hline & 28 & .30 & 33 & 240 & - & 8.00 & .000 & 604 & 70 & - & $<3.0$ & $<1.0$ \\
\hline & 34 & .30 & 34 & 250 & - & 8.70 & .010 & 631 & 70 & - & 4.0 & 1.0 \\
\hline & 31 & .10 & 34 & 240 & - & 5.90 & $<.010$ & 598 & 90 & - & $<3.0$ & $<1.0$ \\
\hline & 34 & .30 & 34 & 250 & - & 9.40 & .020 & 626 & 70 & - & $<3.0$ & $<1.0$ \\
\hline & 36 & .30 & 34 & 250 & - & 9.00 & .020 & 632 & 71 & - & $<3.0$ & $<1.0$ \\
\hline & - & - & - & - & - & 10.5 & $<.050$ & - & - & - & - & - \\
\hline & 37 & .23 & 35 & 260 & $.10 \mathrm{E}$ & 10.4 & $<.050$ & 644 & 60 & .16 & $<10$ & $<2.2$ \\
\hline (C-35-11)12add-1 & 13 & .23 & 48 & 35 & - & .834 & $<.050$ & 303 & 52 & .080 & $<10$ & $<3.0$ \\
\hline \multirow[t]{2}{*}{ (C-35-11)12ccc-2 } & 54 & .27 & 45 & 83 & $.10 \mathrm{E}$ & - & - & 416 & 62 & .26 & 11 & $<3.0$ \\
\hline & 46 & .35 & 45 & 74 & $<.10$ & 10.9 & $<.050$ & 434 & 73 & .22 & $<10$ & $<2.2$ \\
\hline (C-35-11)14bac-2 & 78 & .58 & 31 & 1,600 & .12 & .042 & .06 & 2,510 & 235 & .212 & 30 & 327 \\
\hline \multirow[t]{2}{*}{ (C-35-11)14bca-1 } & 140 & .17 & 35 & 550 & .19 & - & - & 1,210 & 131 & .51 & $<10$ & $<3.0$ \\
\hline & 130 & .38 & 35 & 550 & .19 & 19.5 & $<.050$ & 1,280 & 141 & .52 & $<10$ & $<2.2$ \\
\hline (C-35-11)16aab-1 & 24 & .22 & 23 & 330 & - & 3.28 & $<.050$ & 761 & 67 & .072 & $<10$ & $<3.0$ \\
\hline (C-35-11)19dbb-1 & 15 & .29 & 21 & 270 & .11 & $<.050$ & - & 512 & 102 & .024 & $5.8 \quad \mathrm{E}$ & 5.5 \\
\hline (C-35-11)25bcc-1 & 43 & .25 & 27 & 250 & - & 7.84 & .034 & 616 & 76 & .13 & $<10$ & 111 \\
\hline \multirow[t]{3}{*}{ (C-35-11)26acd-1 } & 42 & .30 & 33 & 220 & - & 14.0 & - & 708 & 130 & - & 20 & $<10$ \\
\hline & 29 & .19 & 33 & 180 & .13 & - & - & 622 & 139 & .12 & $<10$ & $<3.0$ \\
\hline & 27 & .21 & 32 & 180 & $.10 \mathrm{E}$ & 6.59 & $<.050$ & 643 & 139 & .13 & $<10$ & $<2.2$ \\
\hline (C-35-11)27dbb-1 & 11 & .20 & 23 & 130 & .12 & 3.03 & - & 614 & 123 & .036 & $<10$ & $<3.0$ \\
\hline (C-35-11)28aac-2 & 25 & .19 & 23 & 320 & - & 5.18 & $<.050$ & 855 & 71 & .061 & $<10$ & $<3.0$ \\
\hline
\end{tabular}


Table 4. Physical properties and results of chemical analyses of water from selected wells and surface-water sites, Cedar Valley,

\begin{tabular}{|c|c|c|c|c|c|c|c|c|c|c|c|c|}
\hline $\begin{array}{l}\text { Local well } \\
\text { number }\end{array}$ & Date & $\begin{array}{l}\text { pH, water, } \\
\text { whole, } \\
\text { field } \\
\text { (standard } \\
\text { units) }\end{array}$ & $\begin{array}{c}\text { Specific } \\
\text { conduc- } \\
\text { tance } \\
(\mu \mathrm{S} / \mathrm{cm})\end{array}$ & $\begin{array}{c}\text { Water } \\
\text { tempera-ture, } \\
\left({ }^{\circ} \mathrm{C}\right)\end{array}$ & $\begin{array}{c}\text { Hardness, } \\
\text { total } \\
\text { (mg/L as } \\
\mathrm{CaCO}_{3} \text { ) }\end{array}$ & $\begin{array}{c}\text { Calcium, } \\
\text { dissolved } \\
\text { (mg/L as Ca) }\end{array}$ & $\begin{array}{c}\text { Magne-sium, } \\
\text { dissolved } \\
\text { (mg/L as } \mathrm{Mg})\end{array}$ & $\begin{array}{l}\text { Potas- } \\
\text { sium, } \\
\text { dissolved } \\
\text { (mg/L } \\
\text { as K) }\end{array}$ & $\begin{array}{c}\text { Sodium, } \\
\text { dissolved } \\
\text { (mg/L as Na) }\end{array}$ & \multicolumn{2}{|c|}{$\begin{array}{c}\text { Alkalinity, } \\
\text { water, } \\
\text { dissolved } \\
\text { (mg/L as } \\
\left.\mathrm{CaCO}_{3}\right)\end{array}$} & $\begin{array}{c}\text { Bicarbo-nate, } \\
\text { water, } \\
\text { dissolved } \\
(\mathrm{mg} / \mathrm{L} \mathrm{as} \\
\left.\mathrm{HCO}_{3}\right)\end{array}$ \\
\hline \multicolumn{13}{|c|}{ Wells-Continued } \\
\hline \multirow[t]{2}{*}{ (C-35-11)29add-1 } & $08-27-1999$ & 7.5 & 1,610 & 11.5 & 910 & 190 & 106 & 3.3 & 18 & 195 & & 238 \\
\hline & $06-12-2000$ & 7.5 & 1,640 & 16.0 & 980 & 200 & 116 & 4.7 & 19 & 178 & & 217 \\
\hline (C-35-11)29dbd-2 & $07-27-2000$ & 6.9 & 2,160 & 10.5 & 1,300 & 300 & 146 & 5.7 & 31 & 401 & & 500 \\
\hline (C-35-11)30caa-1 & 08-23-1999 & 7.7 & 540 & 14.0 & 270 & 50 & 34 & 2.1 & 14 & 143 & & 174 \\
\hline (C-35-11)31 acd-2 & 08-15-1979 & 7.7 & 630 & 13.0 & 330 & 66 & 39 & 1.9 & 9.1 & 160 & & - \\
\hline \multirow[t]{10}{*}{ (C-35-11)31dbd-1 } & $10-11-1977$ & 8.3 & 750 & 13.5 & - & - & - & - & - & - & & - \\
\hline & $07-18-1980$ & 7.3 & 640 & 12.5 & 350 & 71 & 41 & 2.1 & 9.5 & 160 & & - \\
\hline & 08-04-1983 & 7.7 & 730 & 14.0 & 370 & 77 & 44 & 2.3 & 11 & 167 & $\mathrm{~L}$ & - \\
\hline & 06-27-1986 & 7.6 & 620 & 15.0 & 310 & 62 & 38 & 3.4 & 11 & 152 & $\mathrm{~L}$ & - \\
\hline & 06-04-1991 & 8.0 & 710 & 14.5 & 330 & 63 & 41 & 2.0 & 9.1 & 149 & $\mathrm{~L}$ & - \\
\hline & 06-22-1994 & 7.9 & 680 & 14.0 & 340 & 67 & 41 & 2.4 & 9.3 & 148 & $\mathrm{~L}$ & - \\
\hline & 06-27-1995 & 8.0 & 670 & 13.5 & 330 & 68 & 40 & 2.9 & 9.2 & 148 & $\mathrm{~L}$ & - \\
\hline & 07-10-1998 & 7.3 & 740 & 15.0 & 390 & 75 & 49 & 2.3 & 9.9 & 142 & $\mathrm{~L}$ & - \\
\hline & 07-13-1999 & 7.8 & 800 & 14.5 & 420 & 85 & 50 & 2.2 & 9.9 & 140 & $\mathrm{~L}$ & - \\
\hline & $06-12-2000$ & 8.2 & 870 & 14.5 & 440 & 87 & 54 & 2.3 & 10 & 137 & & 168 \\
\hline \multirow[t]{9}{*}{ (C-35-11)33aac-1 } & $05-22-1974$ & 7.5 & - & 12.5 & 750 & 180 & 74 & 3.6 & 22 & 274 & & - \\
\hline & 06-19-1975 & 7.2 & 1,400 & 11.0 & 830 & 200 & 81 & 3.3 & 22 & 281 & & - \\
\hline & 06-17-1976 & 7.2 & 1,400 & 12.0 & 820 & 190 & 84 & 3.5 & 23 & 297 & & - \\
\hline & 06-13-1977 & 7.2 & 1,350 & 12.5 & 850 & 200 & 86 & 3.6 & 25 & 300 & & - \\
\hline & 08-01-1978 & 6.9 & 1,440 & 12.0 & 820 & 190 & 84 & 3.6 & 23 & 300 & & - \\
\hline & $07-21-1981$ & 7.0 & 1,010 & 13.0 & 600 & 140 & 60 & 2.9 & 17 & 280 & $\mathrm{~L}$ & - \\
\hline & $08-22-1985$ & 7.2 & 1,000 & 11.0 & 580 & 140 & 56 & 2.9 & 15 & 254 & $\mathrm{~L}$ & - \\
\hline & $07-14-1988$ & 7.5 & 1,120 & 12.0 & 670 & 160 & 66 & 3.0 & 15 & 254 & $\mathrm{~L}$ & - \\
\hline & 07-15-1991 & 7.4 & 1,240 & 13.0 & 630 & 150 & 62 & 2.9 & 16 & 274 & $\mathrm{~L}$ & - \\
\hline (C-35-11)33abd-1 & 08-25-1999 & 7.1 & 1,270 & 11.5 & 720 & 170 & 70 & 3.2 & 17 & 332 & & 405 \\
\hline (C-35-11)33ccd-1 & 08-25-1999 & 7.2 & 1,290 & 12.5 & 710 & 150 & 82 & 3.1 & 20 & 230 & & 281 \\
\hline (C-35-11)34dbb-1 & 09-01-1999 & 7.3 & 1,190 & 14.0 & 620 & 130 & 76 & 3.1 & 13 & 312 & & 381 \\
\hline (C-35-12)26bca-1 & 07-21-1999 & 7.2 & 1,050 & 12.5 & 520 & 99 & 65 & 2.8 & 41 & 170 & $\mathrm{~L}$ & - \\
\hline (C-35-12)36caa-1 & 08-23-1999 & 7.5 & 650 & 16.5 & 290 & 54 & 39 & 2.9 & 23 & 133 & & 162 \\
\hline (C-35-12)36caa-2 & 03-14-2001 & 7.1 & 3,580 & 12.0 & 2,300 & 360 & 326 & 3.9 & 1.0 & 267 & & 325 \\
\hline (C-35-12)36ddd-1 & 08-26-1999 & 7.9 & 540 & 14.0 & 260 & 50 & 33 & 2.2 & 15 & 162 & & 197 \\
\hline \multirow[t]{2}{*}{ (C-36-11) 5aca-1 } & 05-23-1974 & 7.6 & 1,300 & 11.0 & 820 & 180 & 90 & 4.2 & 23 & 279 & & - \\
\hline & 08-26-1999 & 7.0 & 1,780 & 11.5 & 1,100 & 230 & 116 & 4.0 & 28 & 320 & & 390 \\
\hline (C-36-11) 5dab-1 & 09-01-1999 & 7.3 & 1,090 & 12.5 & 590 & 120 & 69 & 3.5 & 23 & 310 & & 478 \\
\hline (C-36-11) 7aaa-2 & 09-01-1999 & 7.2 & 1,410 & 13.5 & 800 & 160 & 97 & 3.6 & 28 & 312 & & 381 \\
\hline (C-36-11) 7cab-1 & 08-23-1999 & 7.8 & 1,060 & 15.5 & 590 & 120 & 73 & 3.4 & 16 & 140 & & 171 \\
\hline \multirow[t]{2}{*}{ (C-36-11)11bac-1 } & 08-26-1999 & 7.7 & 2,220 & 14.5 & 1,400 & 300 & 148 & 4.1 & 35 & 238 & & 291 \\
\hline & $07-25-2000$ & 7.4 & 2,190 & 16.5 & 1,300 & 290 & 147 & 4.2 & 36 & 236 & & 288 \\
\hline (C-36-11)18bdd-1 & 07-22-1999 & 7.5 & 1,440 & 16.5 & 700 & 150 & 83 & 3.6 & 66 & 182 & & 222 \\
\hline (C-36-11)31abc-1 & $07-28-2000$ & 7.5 & 1,140 & - & 550 & 140 & 49 & 2.6 & 16 & 202 & & 247 \\
\hline (C-36-12) $2 \mathrm{dbc}-1$ & 07-21-1999 & 7.5 & 600 & 19.5 & 280 & 56 & 34 & 2.6 & 20 & 140 & & - \\
\hline (C-36-12) 3aad-2 & 07-21-1999 & 7.8 & 530 & 16.0 & 300 & 62 & 36 & 2.6 & 23 & 160 & $\mathrm{~L}$ & - \\
\hline (C-36-12) 9aac-1 & 07-21-1999 & 7.6 & 540 & - & 230 & 53 & 25 & 3.0 & 19 & 130 & $\mathrm{~L}$ & - \\
\hline \multirow[t]{8}{*}{$(\mathrm{C}-36-12) 12 \mathrm{dba}-1$} & 09-05-1961 & 7.6 & 710 & 20.0 & 380 & 86 & 40 & - & - & 145 & & - \\
\hline & 09-10-1969 & 7.7 & 550 & 13.0 & 280 & 47 & 39 & - & - & 153 & & - \\
\hline & $05-22-1974$ & 8.0 & 600 & 14.0 & 280 & 52 & 36 & 2.4 & 8.5 & 144 & & - \\
\hline & 06-28-1982 & 8.1 & 560 & 16.0 & 270 & 52 & 34 & 2.4 & 8.8 & 149 & $\mathrm{~L}$ & - \\
\hline & 06-24-1986 & 7.4 & 590 & 15.0 & 270 & 53 & 34 & 2.2 & 8.6 & 148 & $\mathrm{~L}$ & - \\
\hline & 07-05-1990 & 7.8 & 570 & 15.5 & 280 & 55 & 35 & 2.5 & 11 & 147 & $\mathrm{~L}$ & - \\
\hline & 06-07-1991 & 7.8 & 590 & 15.5 & 290 & 56 & 37 & 2.2 & 8.9 & 148 & $\mathrm{~L}$ & - \\
\hline & 08-23-1994 & 7.8 & 580 & 14.0 & 280 & 55 & 34 & 3.2 & 8.7 & 149 & $\mathrm{~L}$ & - \\
\hline
\end{tabular}


Iron County, southwestern Utah-Continued

\begin{tabular}{|c|c|c|c|c|c|c|c|c|c|c|c|c|c|}
\hline $\begin{array}{l}\text { Local well } \\
\text { number }\end{array}$ & $\begin{array}{c}\text { Chloride, } \\
\text { dis- } \\
\text { solved } \\
\text { (mg/L as Cl) }\end{array}$ & $\begin{array}{l}\text { Fluoride, dis- } \\
\text { solved } \\
\text { (mg/L } \\
\text { as F) }\end{array}$ & $\begin{array}{c}\text { Silica, } \\
\text { dissolved } \\
(\mathrm{mg} / \mathrm{L} \\
\left.\text { as } \mathrm{SiO}_{2}\right)\end{array}$ & $\begin{array}{c}\text { Sulfate, } \\
\text { dissolved } \\
(\mathrm{mg} / \mathrm{L} \\
\left.\text { as } \mathrm{SO}_{4}\right)\end{array}$ & $\begin{array}{c}\text { Nitrogen, } \\
\text { ammonia + } \\
\text { organic, } \\
\text { dissolved } \\
\text { (mg/L } \\
\text { as N) }\end{array}$ & $\begin{array}{c}\text { Nitrogen, } \\
\mathrm{NO}_{2}+\mathrm{NO}_{3}, \\
\text { dissolved } \\
\text { (mg/L } \\
\text { as N) }\end{array}$ & $\begin{array}{l}\text { Phos-phorus, } \\
\text { dissolved } \\
\text { (mg/L } \\
\text { as P) }\end{array}$ & $\begin{array}{l}\text { Solids, sum } \\
\text { of consti- } \\
\text { tuents, } \\
\text { dissolved } \\
\text { (mg/L) }\end{array}$ & $\begin{array}{c}\text { Boron, } \\
\text { dis- } \\
\text { solved } \\
\text { ( } \mu \mathrm{g} / \mathrm{L} \\
\text { as B) }\end{array}$ & $\begin{array}{c}\text { Bromide, } \\
\text { dis- } \\
\text { solved } \\
\text { (mg/L } \\
\text { as Br) }\end{array}$ & $\begin{array}{l}\text { Iron, } \\
\text { dis- } \\
\text { solved } \\
(\mu \mathrm{g} / \mathrm{L} \\
\text { as Fe })\end{array}$ & & $\begin{array}{c}\text { Manga- } \\
\text { nese, } \\
\text { dis- } \\
\text { solved } \\
(\mu \mathrm{g} / \mathrm{L} \\
\text { as } \mathrm{Mn})\end{array}$ \\
\hline \multicolumn{14}{|c|}{ Wells-Continued } \\
\hline \multirow[t]{2}{*}{ (C-35-11)29add-1 } & 24 & .17 & 21 & 690 & .10 & - & $<.050$ & 1,170 & 47 & .073 & $<10$ & & $<3.0$ \\
\hline & 27 & .17 & 22 & 770 & - & 6.81 & $<.050$ & 1,300 & 36 & .082 & $<10$ & & $<2.2$ \\
\hline (C-35-11)29dbd-2 & 35 & .21 & 21 & 1,000 & .14 & 2.45 & $<.050$ & 1,790 & 93 & .087 & $<30$ & & $<2.2$ \\
\hline (C-35-11)30caa-1 & 11 & .28 & 23 & 130 & - & .756 & $<.050$ & 346 & 37 & .041 & 7.5 & E & $<3.0$ \\
\hline (C-35-11)31 acd-2 & 10 & .30 & 21 & 170 & - & .970 & .010 & 418 & 30 & - & - & & \\
\hline \multirow[t]{10}{*}{ (C-35-11)31dbd-1 } & - & - & - & - & - & 1.70 & - & - & - & - & - & & - \\
\hline & 11 & .30 & 20 & 170 & - & 1.10 & .050 & 425 & 70 & - & - & & - \\
\hline & 14 & .30 & 22 & 200 & - & 1.20 & .020 & 476 & 30 & - & 29 & & 4.0 \\
\hline & 12 & .30 & 20 & 170 & - & 1.10 & .010 & 413 & 40 & - & $<3.0$ & & 3.0 \\
\hline & 14 & .20 & 20 & 180 & - & - & - & 419 & 40 & - & $<3.0$ & & $<1.0$ \\
\hline & 14 & .30 & 22 & 190 & - & 1.40 & .070 & 441 & 60 & - & 4.0 & & $<1.0$ \\
\hline & 14 & .30 & 21 & 190 & - & 1.50 & .680 & 441 & 30 & - & $<3.0$ & & $<1.0$ \\
\hline & 15 & .32 & 22 & 230 & - & 1.83 & .048 & 497 & 37 & - & $<10$ & & $<4.0$ \\
\hline & 16 & .27 & 20 & 270 & - & 2.00 & - & 541 & 50 & - & 8.9 & $\mathrm{E}$ & $<3.0$ \\
\hline & 16 & .23 & 22 & 290 & - & 2.17 & $<.050$ & 578 & 31 & .075 & $<10$ & & $<2.2$ \\
\hline \multirow[t]{9}{*}{ (C-35-11)33aac-1 } & 19 & .20 & 17 & 470 & - & 4.00 & - & 968 & 60 & - & - & & - \\
\hline & 16 & .20 & 17 & 500 & - & 4.20 & - & 1,030 & 50 & - & - & & - \\
\hline & 17 & .40 & 16 & 530 & - & 4.70 & - & 1,060 & 60 & - & - & & - \\
\hline & 16 & .20 & 18 & 510 & - & 4.70 & - & 1,060 & 60 & - & - & & - \\
\hline & 17 & .20 & 17 & 490 & - & 4.40 & - & 1,030 & 60 & - & - & & - \\
\hline & 18 & .10 & 18 & 340 & - & 2.50 & .030 & 775 & 40 & - & $<10$ & & 2.0 \\
\hline & 14 & .20 & 16 & 310 & - & 4.90 & $<.010$ & 728 & 50 & - & 4.0 & & $<1.0$ \\
\hline & 15 & .20 & 16 & 390 & - & 6.50 & .020 & 846 & 50 & - & $<3.0$ & & $<1.0$ \\
\hline & 16 & .20 & 16 & 370 & - & - & - & 797 & 60 & - & 6.0 & & $<1.0$ \\
\hline (C-35-11)33abd-1 & 21 & .16 & 20 & 360 & .47 & 5.03 & - & 860 & 60 & .044 & $<10$ & & $<3.0$ \\
\hline$(\mathrm{C}-35-11) 33 \mathrm{ccd}-1$ & 13 & .23 & 20 & 480 & .32 & 4.83 & - & 903 & 118 & .055 & 7.5 & E & $<3.0$ \\
\hline (C-35-11)34dbb-1 & 120 & .21 & 22 & 150 & - & 8.98 & $<.050$ & 735 & 99 & .11 & $<10$ & & $<3.0$ \\
\hline (C-35-12)26bca-1 & 34 & .47 & 22 & 360 & - & .337 & $<.050$ & 730 & 119 & .14 & 8.9 & $\mathrm{E}$ & 2.0 \\
\hline (C-35-12)36саa-1 & 12 & .29 & 29 & 190 & .17 & .277 & - & 427 & 83 & .020 & $<10$ & & $<3.0$ \\
\hline (C-35-12)36саa-2 & 119 & .70 & 23 & 1,970 & .23 & 2.67 & $.011 \mathrm{E}$ & 3,070 & 391 & .31 & 20 & $\mathrm{E}$ & 708 \\
\hline (C-35-12)36ddd-1 & 6.6 & .31 & 23 & 120 & - & .248 & $<.050$ & 343 & 52 & .022 & $<10$ & & 8.8 \\
\hline \multirow[t]{2}{*}{ (C-36-11) 5aca-1 } & 26 & .20 & 20 & 500 & - & 7.30 & - & 1,040 & 100 & - & 30 & & $<10$ \\
\hline & 36 & .21 & 22 & 700 & - & 5.46 & $<.050$ & 1,350 & 95 & .11 & $<10$ & & $<3.0$ \\
\hline (C-36-11) 5dab-1 & 23 & .25 & 21 & 290 & - & 3.52 & $<.050$ & 797 & 109 & .067 & 8.5 & E & $<3.0$ \\
\hline (C-36-11) 7aaa-2 & 36 & .22 & 26 & 490 & - & 3.95 & $<.050$ & 1,040 & 100 & .095 & $<10$ & & $<3.0$ \\
\hline (C-36-11) 7cab-1 & 25 & .27 & 25 & 410 & .25 & 4.41 & - & 754 & 30 & .093 & 9.0 & E & $<3.0$ \\
\hline \multirow[t]{2}{*}{ (C-36-11)11bac-1 } & 31 & .21 & 23 & 1,100 & - & 6.48 & $<.050$ & 1,810 & 242 & .11 & $<30$ & & $<6.6$ \\
\hline & 29 & .23 & 23 & 1,100 & - & 6.51 & $<.050$ & 1,820 & 270 & .10 & $<30$ & & $<2.2$ \\
\hline (C-36-11)18bdd-1 & 61 & .25 & 33 & 530 & - & 4.05 & $<.050$ & 1,050 & 176 & .23 & $<10$ & & $<3.0$ \\
\hline (C-36-11)31 abc- 1 & 22 & .15 & 24 & 350 & $<.10$ & 7.50 & $<.050$ & 758 & 75 & .13 & $<10$ & & $1.4 \mathrm{E}$ \\
\hline (C-36-12) 2dbc-1 & 8.4 & .32 & 24 & 160 & - & .432 & $<.050$ & 396 & 81 & .039 & $<10$ & & $<3.0$ \\
\hline (C-36-12) 3aad-2 & 11 & .30 & 24 & 180 & - & .606 & $<.050$ & 438 & 92 & .041 & $<10$ & & $<3.0$ \\
\hline (C-36-12) 9aac-1 & 10 & .15 & 33 & 130 & - & .386 & $<.050$ & 352 & 67 & .054 & $<10$ & & $<3.0$ \\
\hline \multirow[t]{8}{*}{ (C-36-12)12dba-1 } & 17 & - & 22 & 230 & - & - & - & 508 & - & - & - & & - \\
\hline & 13 & - & - & 120 & - & - & - & 319 & - & - & - & & - \\
\hline & 12 & $<.10$ & 21 & 130 & - & 1.10 & - & 354 & 30 & - & $<10$ & & $<10$ \\
\hline & 10 & 1.1 & 7.3 & 120 & - & .680 & $<.010$ & 351 & 30 & - & 9.0 & & 3.0 \\
\hline & 15 & .40 & 21 & 120 & - & 1.60 & .010 & 350 & 40 & - & $<3.0$ & & 5.0 \\
\hline & 15 & .20 & 23 & 130 & - & 1.60 & .020 & 367 & 60 & - & 8.0 & & $<1.0$ \\
\hline & 16 & .30 & 21 & 140 & - & - & - & 370 & 40 & - & 9.0 & & $<1.0$ \\
\hline & 14 & .20 & 23 & 130 & - & 1.70 & .020 & 365 & 50 & - & 3.0 & & 6.0 \\
\hline
\end{tabular}


Table 4. Physical properties and results of chemical analyses of water from selected wells and surface-water sites, Cedar Valley,

\begin{tabular}{|c|c|c|c|c|c|c|c|c|c|c|c|c|}
\hline $\begin{array}{c}\text { Local well } \\
\text { number }\end{array}$ & Date & $\begin{array}{l}\text { pH, water, } \\
\text { whole, } \\
\text { field } \\
\text { (standard } \\
\text { units) }\end{array}$ & $\begin{array}{c}\text { Specific } \\
\text { conduc- } \\
\text { tance } \\
(\mu \mathrm{S} / \mathrm{cm})\end{array}$ & $\begin{array}{c}\text { Water } \\
\text { tempera- } \\
\text { ture, } \\
\left({ }^{\circ} \mathrm{C}\right)\end{array}$ & $\begin{array}{c}\text { Hardness, } \\
\text { total } \\
(\mathrm{mg} / \mathrm{L} \text { as } \\
\left.\mathrm{CaCO}_{3}\right)\end{array}$ & $\begin{array}{c}\text { Calcium, } \\
\text { dissolved } \\
\text { (mg/L as Ca) }\end{array}$ & $\begin{array}{l}\text { Magne-sium, } \\
\text { dissolved } \\
\text { (mg/L as } \mathrm{Mg} \text { ) }\end{array}$ & $\begin{array}{l}\text { Potas- } \\
\text { sium, } \\
\text { dissolved } \\
\text { (mg/L } \\
\text { as K) }\end{array}$ & $\begin{array}{c}\text { Sodium, } \\
\text { dissolved } \\
\text { (mg/L as Na) }\end{array}$ & \multicolumn{2}{|c|}{$\begin{array}{c}\text { Alkalinity, } \\
\text { water, } \\
\text { dissolved(mg } \\
\left./ \mathrm{L} \text { as } \mathrm{CaCO}_{3}\right)\end{array}$} & $\begin{array}{c}\text { Bicarbo-nate, } \\
\text { water, } \\
\text { dissolved } \\
(\mathrm{mg} / \mathrm{L} \text { as } \\
\left.\mathrm{HCO}_{3}\right)\end{array}$ \\
\hline \multicolumn{13}{|c|}{ Wells-Continued } \\
\hline \multirow[t]{2}{*}{ (C-36-12)12dba-1 } & 05-20-1997 & 7.8 & 590 & 14.5 & 280 & 54 & 36 & 2.2 & 8.6 & 146 & $\mathrm{~L}$ & - \\
\hline & 08-23-1999 & 7.7 & 550 & 14.5 & 290 & 56 & 37 & 2.1 & 8.9 & 143 & & 174 \\
\hline \multirow[t]{6}{*}{ (C-36-12)21cbb-1 } & 05-22-1974 & 8.2 & 350 & 12.5 & 160 & 39 & 14 & 2.2 & 12 & 112 & & - \\
\hline & 07-19-1979 & 8.0 & 350 & 14.5 & 160 & 41 & 13 & 3.2 & 11 & 120 & & - \\
\hline & $07-18-1980$ & 7.7 & 335 & 14.0 & 150 & 40 & 13 & 2.5 & 11 & 130 & & - \\
\hline & $05-27-1981$ & 7.9 & 320 & 14.0 & 160 & 42 & 13 & 2.9 & 11 & 120 & $\mathrm{~L}$ & - \\
\hline & 06-30-1982 & 8.0 & 345 & 14.0 & 150 & 41 & 12 & 2.3 & 11 & 124 & $\mathrm{~L}$ & - \\
\hline & 06-08-1988 & 8.0 & 340 & 14.0 & 170 & 43 & 14 & 6.1 & 13 & 125 & $\mathrm{~L}$ & - \\
\hline \multirow[t]{2}{*}{ (C-36-12)36adb-1 } & 07-22-1999 & 7.2 & 860 & - & 470 & 120 & 43 & 2.1 & 16 & 300 & & 366 \\
\hline & $07-25-2000$ & 7.1 & 880 & 14.5 & 450 & 110 & 42 & 1.9 & 15 & 206 & $\mathrm{~L}$ & - \\
\hline (C-37-12) 5acc-2 & 08-23-1999 & 7.4 & 265 & 15.5 & 110 & 33 & 6.1 & 1.9 & 11 & 306 & & 129 \\
\hline \multirow[t]{6}{*}{ (C-37-12) 9acc-1 } & 09-11-1974 & - & 360 & 16.0 & 140 & 48 & 5.8 & 3.8 & 14 & 136 & & - \\
\hline & 08-04-1983 & 7.5 & 350 & 16.0 & 150 & 50 & 6.3 & 3.5 & 15 & 138 & $\mathrm{~L}$ & - \\
\hline & 07-22-1992 & 7.8 & 355 & 16.5 & 150 & 50 & 6.2 & 3.2 & 15 & 137 & $\mathrm{~L}$ & - \\
\hline & 06-23-1994 & 7.7 & 365 & 16.5 & 140 & 48 & 6.0 & 3.4 & 14 & 136 & $\mathrm{~L}$ & - \\
\hline & 05-20-1997 & 7.6 & 365 & 16.0 & 140 & 47 & 5.8 & 3.4 & 14 & 136 & $\mathrm{~L}$ & - \\
\hline & 07-13-1999 & 7.9 & 375 & 16.5 & 150 & 51 & 6.4 & 3.4 & 14 & 130 & $\mathrm{~L}$ & - \\
\hline (C-37-12)11dac-1 & 08-23-1999 & 7.6 & 640 & 18.5 & 260 & 55 & 31 & 5.4 & 34 & 129 & & 157 \\
\hline \multirow[t]{5}{*}{ (C-37-12)23abd-1 } & 06-04-1991 & 7.9 & 650 & 16.5 & 240 & 57 & 24 & 1.5 & 35 & 157 & $\mathrm{~L}$ & - \\
\hline & 07-20-1993 & 7.8 & 600 & 17.5 & 240 & 55 & 24 & 1.4 & 34 & 154 & $\mathrm{~L}$ & - \\
\hline & 06-23-1994 & 7.7 & 680 & 16.5 & 270 & 63 & 28 & 1.5 & 33 & 182 & $\mathrm{~L}$ & - \\
\hline & 05-20-1997 & 7.6 & 630 & 16.0 & 240 & 55 & 26 & 1.6 & 34 & 161 & $\mathrm{~L}$ & - \\
\hline & 07-13-1999 & 7.4 & 640 & 17.5 & 270 & 62 & 27 & 1.4 & 35 & 160 & $\mathrm{~L}$ & - \\
\hline \multirow[t]{5}{*}{ (C-37-12)23acb-1 } & 05-22-1974 & 7.8 & 600 & 13.5 & 240 & 55 & 26 & 1.5 & 32 & 133 & & - \\
\hline & 07-19-1979 & 7.7 & 780 & 14.5 & 320 & 73 & 34 & 1.8 & 37 & 120 & & - \\
\hline & 05-28-1981 & 7.7 & 780 & 13.5 & 320 & 72 & 34 & 1.9 & 37 & 130 & $\mathrm{~L}$ & - \\
\hline & 08-31-1984 & 7.7 & 760 & 14.5 & 340 & 75 & 36 & 2.3 & 37 & 139 & $\mathrm{~L}$ & - \\
\hline & $07-19-1990$ & 7.7 & 810 & 15.5 & 330 & 77 & 34 & 1.9 & 38 & 149 & $\mathrm{~L}$ & - \\
\hline \multirow[t]{14}{*}{ (C-37-12)34abb-1 } & 09-05-1961 & 7.8 & 690 & 11.5 & 360 & 88 & 33 & - & - & 220 & & - \\
\hline & 09-01-1964 & 7.7 & 780 & 11.5 & 420 & 110 & 38 & - & 20 & 284 & & - \\
\hline & 09-01-1967 & 7.7 & 780 & 20.0 & 420 & 110 & 38 & - & - & 284 & & - \\
\hline & 05-12-1972 & 7.2 & 890 & 12.0 & 460 & 120 & 40 & 1.8 & 17 & 306 & & - \\
\hline & 05-22-1974 & 7.6 & 800 & 11.5 & 460 & 120 & 40 & 2.3 & 16 & 308 & & - \\
\hline & 08-15-1979 & 7.0 & 910 & 12.0 & 460 & 120 & 40 & 2.1 & 17 & 330 & & - \\
\hline & 07-30-1982 & 7.7 & 910 & 11.0 & 460 & 120 & 40 & 2.1 & 17 & 167 & $\mathrm{~L}$ & - \\
\hline & 08-31-1984 & 7.6 & 810 & 11.0 & 460 & 120 & 39 & 2.5 & 16 & 340 & $\mathrm{~L}$ & - \\
\hline & 07-15-1988 & $7.5 \quad \mathrm{~L}$ & 810 & 12.0 & 470 & 120 & 41 & 2.1 & 16 & 307 & $\mathrm{~L}$ & - \\
\hline & 07-11-1989 & 7.2 & 840 & 12.0 & 430 & 110 & 38 & 2.2 & 17 & 336 & $\mathrm{~L}$ & - \\
\hline & 06-04-1991 & 7.3 & 790 & 11.5 & 460 & 120 & 40 & 2.1 & 16 & 324 & $\mathrm{~L}$ & - \\
\hline & 06-23-1994 & 7.2 & 840 & 12.0 & 430 & 110 & 37 & 2.0 & 14 & 335 & $\mathrm{~L}$ & - \\
\hline & 07-01-1997 & 7.2 & 780 & 12.5 & 410 & 110 & 34 & 2.0 & 14 & 317 & $\mathrm{~L}$ & - \\
\hline & 06-13-2000 & 7.1 & 810 & 11.0 & 410 & 100 & 35 & 2.0 & 15 & 311 & & 379 \\
\hline \multicolumn{13}{|c|}{ Surface-water sites } \\
\hline \multirow[t]{3}{*}{ Coal Creek } & 07-27-1999 & 8.1 & 445 & 18.0 & 220 & 52 & 22 & 1.5 & 6.7 & 170 & & 158 \\
\hline & 03-16-2000 & 8.4 & 500 & 9.0 & 250 & 62 & 24 & 1.9 & 8.8 & 190 & & 159 \\
\hline & 06-13-2000 & 8.2 & 420 & 22.0 & 200 & 48 & 21 & 1.6 & 6.5 & 166 & & 154 \\
\hline (C-35-11)36bac & 07-27-1999 & 8.5 & 590 & 24.0 & 260 & 60 & 26 & 3.5 & 19 & 140 & & 122 \\
\hline (C-36-11)31bda & 07-15-1999 & 7.8 & 510 & 21.0 & 250 & 68 & 18 & 1.4 & 11 & 132 & $\mathrm{~L}$ & - \\
\hline
\end{tabular}


Iron County, southwestern Utah—Continued

\begin{tabular}{|c|c|c|c|c|c|c|c|c|c|c|c|c|}
\hline $\begin{array}{l}\text { Local well } \\
\text { number }\end{array}$ & $\begin{array}{c}\text { Chloride, } \\
\text { dis- } \\
\text { solved } \\
\text { (mg/L as Cl) }\end{array}$ & $\begin{array}{l}\text { Fluoride, dis- } \\
\text { solved } \\
\text { (mg/L } \\
\text { as F) }\end{array}$ & $\begin{array}{c}\text { Silica, } \\
\text { dissolved } \\
(\mathrm{mg} / \mathrm{L} \\
\left.\text { as } \mathrm{SiO}_{2}\right)\end{array}$ & $\begin{array}{c}\text { Sulfate, } \\
\text { dissolved } \\
\text { (mg/L } \\
\left.\text { as } \mathrm{SO}_{4}\right)\end{array}$ & $\begin{array}{c}\text { Nitrogen, } \\
\text { ammonia + } \\
\text { organic, } \\
\text { dissolved } \\
\text { (mg/L } \\
\text { as N) }\end{array}$ & $\begin{array}{c}\text { Nitrogen, } \\
\mathrm{NO}_{2}+\mathrm{NO}_{3} \\
\text { dissolved } \\
\text { (mg/L } \\
\text { as } \mathrm{N})\end{array}$ & $\begin{array}{c}\text { Phos-phorus, } \\
\text { dissolved } \\
\text { (mg/L } \\
\text { as } \mathrm{P})\end{array}$ & $\begin{array}{l}\text { Solids, sum } \\
\text { of consti- } \\
\text { tuents, } \\
\text { dissolved } \\
\text { (mg/L) }\end{array}$ & $\begin{array}{c}\text { Boron, } \\
\text { dis- } \\
\text { solved } \\
(\mu \mathrm{g} / \mathrm{L} \\
\text { as B) }\end{array}$ & $\begin{array}{c}\text { Bromide, } \\
\text { dis- } \\
\text { solved } \\
\text { (mg/L } \\
\text { as } \mathrm{Br} \text { ) }\end{array}$ & $\begin{array}{c}\text { Iron, } \\
\text { dis- } \\
\text { solved } \\
(\mu \mathrm{g} / \mathrm{L} \\
\text { as Fe) }\end{array}$ & $\begin{array}{c}\text { Manga- } \\
\text { nese, } \\
\text { dis- } \\
\text { solved } \\
(\mu \mathrm{g} / \mathrm{L} \\
\text { as } \mathrm{Mn})\end{array}$ \\
\hline \multicolumn{13}{|c|}{ Wells-Continued } \\
\hline \multirow[t]{2}{*}{ (C-36-12)12dba-1 } & 16 & .36 & 22 & 140 & - & 1.74 & $<.010$ & 375 & 40 & - & $<3.0$ & $<1.0$ \\
\hline & 14 & .34 & 24 & 130 & .15 & 1.49 & - & 362 & 28 & .060 & $<10$ & $<3.0$ \\
\hline \multirow[t]{6}{*}{ (C-36-12)21cbb-1 } & 9.5 & .20 & 42 & 57 & - & .540 & - & 245 & 30 & - & - & - \\
\hline & 9.6 & .20 & 44 & 42 & - & .480 & .060 & 238 & 50 & - & - & - \\
\hline & 9.3 & .03 & 43 & 37 & - & .410 & .030 & 235 & 50 & - & 10 & - \\
\hline & 15 & .20 & 42 & 40 & - & .310 & .040 & 240 & 30 & - & 20 & 2.0 \\
\hline & 9.3 & .40 & 44 & 38 & - & .350 & $<.010$ & 234 & 40 & - & 13 & 1.0 \\
\hline & 11 & .30 & 45 & 59 & - & .270 & .020 & 268 & 40 & - & 22 & 7.0 \\
\hline \multirow[t]{2}{*}{ (C-36-12)36adb-1 } & 8.0 & .12 & 18 & 170 & - & 1.95 & $<.050$ & 567 & 95 & .10 & $<10$ & $<3.0$ \\
\hline & 7.2 & .11 & 20 & 180 & - & 1.98 & $<.050$ & 570 & 86 & .064 & $<10$ & $<2.2$ \\
\hline$(\mathrm{C}-37-12) 5 \mathrm{acc}-2$ & 13 & $<.10$ & 50 & 3.5 & $.10 \mathrm{E}$ & .999 & - & 183 & 22 & .073 & $5.2 \mathrm{E}$ & $<3.0$ \\
\hline \multirow[t]{6}{*}{$(\mathrm{C}-37-12) 9 \mathrm{acc}-1$} & 21 & .20 & 54 & 12 & - & .880 & - & 245 & 40 & - & 20 & $<10$ \\
\hline & 22 & .30 & 53 & 18 & - & .880 & .010 & 255 & 40 & - & $<3.0$ & $<1.0$ \\
\hline & 26 & .30 & 57 & 11 & - & 1.00 & .020 & 255 & 50 & - & $<3.0$ & $<1.0$ \\
\hline & 26 & .20 & 55 & 11 & - & 1.00 & .040 & 250 & 50 & - & $<3.0$ & $<1.0$ \\
\hline & 26 & .21 & 59 & 11 & - & 1.04 & $<.010$ & 253 & 46 & - & $<3.0$ & $<1.0$ \\
\hline & 26 & .20 & 52 & 11 & - & 1.06 & $<.050$ & 248 & 48 & - & $<10$ & $<3.0$ \\
\hline (C-37-12)11dac-1 & 31 & .18 & 48 & 150 & .21 & 1.06 & - & 433 & 114 & .17 & 10 & $1.6 \mathrm{E}$ \\
\hline \multirow[t]{5}{*}{ (C-37-12)23abd-1 } & 32 & .10 & 18 & 120 & - & - & - & 382 & 90 & - & 5.0 & $<1.0$ \\
\hline & 31 & .20 & 18 & 110 & - & 2.70 & .020 & 378 & 90 & - & 3.0 & 1.0 \\
\hline & 36 & .10 & 20 & 110 & - & 3.50 & .050 & 416 & 110 & - & 6.0 & 1.0 \\
\hline & 35 & .10 & 18 & 110 & - & 2.92 & $<.010$ & 389 & 108 & - & $<3.0$ & $<1.0$ \\
\hline & 36 & .10 & 19 & 110 & - & 2.96 & - & 400 & 92 & - & $<10$ & $<3.0$ \\
\hline \multirow[t]{5}{*}{ (C-37-12)23acb-1 } & 26 & $<.10$ & 17 & 140 & - & .700 & - & 381 & 60 & - & 20 & $<10$ \\
\hline & 41 & .10 & 17 & 200 & - & .970 & .030 & 480 & 70 & - & - & - \\
\hline & 39 & .10 & 17 & 200 & - & 1.20 & .030 & 485 & 50 & - & $<10$ & $<1.0$ \\
\hline & 49 & $<.10$ & 18 & 200 & - & 1.40 & $<.010$ & 507 & 90 & - & 5.0 & $<1.0$ \\
\hline & 49 & $<.10$ & 19 & 190 & - & 1.80 & .010 & 506 & 100 & - & 12 & 1.0 \\
\hline \multirow[t]{14}{*}{ (C-37-12)34abb-1 } & 13 & - & 16 & 150 & - & - & - & 477 & - & - & - & - \\
\hline & 73 & - & 16 & 150 & - & - & - & 585 & - & - & - & - \\
\hline & 13 & - & 16 & 150 & - & - & - & 525 & - & - & - & - \\
\hline & 13 & - & 18 & 150 & - & - & - & 543 & - & - & - & - \\
\hline & 11 & - & 17 & 150 & - & - & - & 542 & - & - & - & - \\
\hline & 10 & .20 & 18 & 150 & - & 2.40 & .020 & 566 & 70 & - & - & - \\
\hline & 10 & .20 & 18 & 150 & - & 2.20 & .030 & 468 & 70 & - & 4.0 & $<1.0$ \\
\hline & 9.2 & .20 & 18 & 150 & - & 1.70 & $<.010$ & 566 & 90 & - & 3.0 & 1.0 \\
\hline & 10 & .20 & 18 & 150 & - & 1.70 & .050 & 549 & 60 & - & $<3.0$ & $<1.0$ \\
\hline & 9.3 & .20 & 17 & 140 & - & 1.90 & $<.010$ & 544 & 70 & - & 5.0 & $<1.0$ \\
\hline & 11 & .20 & 17 & 150 & - & - & - & 551 & 70 & - & 5.0 & 2.0 \\
\hline & 8.5 & .20 & 18 & 130 & - & 1.20 & .030 & 526 & 100 & - & 4.0 & $<1.0$ \\
\hline & 7.8 & .17 & 17 & 120 & - & .962 & $<.010$ & 503 & 62 & - & $<3.0$ & $<1.0$ \\
\hline & 7.3 & .19 & 18 & 120 & - & 1.10 & $<.050$ & 497 & 57 & .043 & $<10$ & $<2.2$ \\
\hline \multicolumn{13}{|c|}{ Surface-water sites } \\
\hline \multirow[t]{3}{*}{ Coal Creek } & 7.3 & .20 & 11 & 60 & - & .068 & $<.050$ & 238 & 32 & .01 & $<10$ & $2 \mathrm{E}$ \\
\hline & 10 & .20 & 10 & 73 & - & .163 & $<.050$ & 270 & 33 & .01 & $<10$ & $2 \mathrm{E}$ \\
\hline & 6.9 & .20 & 12 & 50 & - & $<.050$ & $<.050$ & 222 & 19 & $<.01$ & $<10$ & $<2$ \\
\hline$(C-35-11) 36 b a c$ & 22 & .20 & 21 & 134 & - & $<.050$ & $<.050$ & 346 & 53 & .05 & 80 & 5 \\
\hline (C-36-11)31bda & 6.0 & $<.1$ & 27 & 119 & - & .089 & $<.050$ & 330 & 45 & - & $<10$ & 3 \\
\hline
\end{tabular}


Table 5. Discharge, time of measurement, and temperature of water from Coal Creek, Quichapa Ditch, and Shirts Creek, Cedar Valley, Iron County, southwestern Utah

$[-$, no data available $]$

Site ID: Informal site identification is designated by letters and measurement numbers, listed in downstream order. CC, Coal Creek; CQ, Coal Creek-Quichapa Ditch; CW, Coal Creek downstream of Woodbury Diversion; SC, Shirts Creek.

Location: See figure 2 for an explanation of the numbering system used for hydrologic-data sites in Utah. See plate 3 for location of measurement sites.

Description: Location of discharge measurement. Measurements made by current-meter except where noted. ft, feet; USGS, U.S. Geological Survey; SR, state road; mi, mile; I, interstate highway.

Discharge: $\mathrm{ft}^{3} / \mathrm{s}$, cubic feet per second; e, estimated; dry, no flow.

Time: Time of day discharge measurement was made.

Water temperature: ${ }^{\circ} \mathrm{C}$, degrees Celsius.

Remarks: Percent error in measured discharge: Good, plus or minus 5 percent; Fair, plus or minus 8 percent; Poor, greater than 8 percent. K, specific conductance in microsiemens per centimeter at $25^{\circ} \mathrm{C}$.

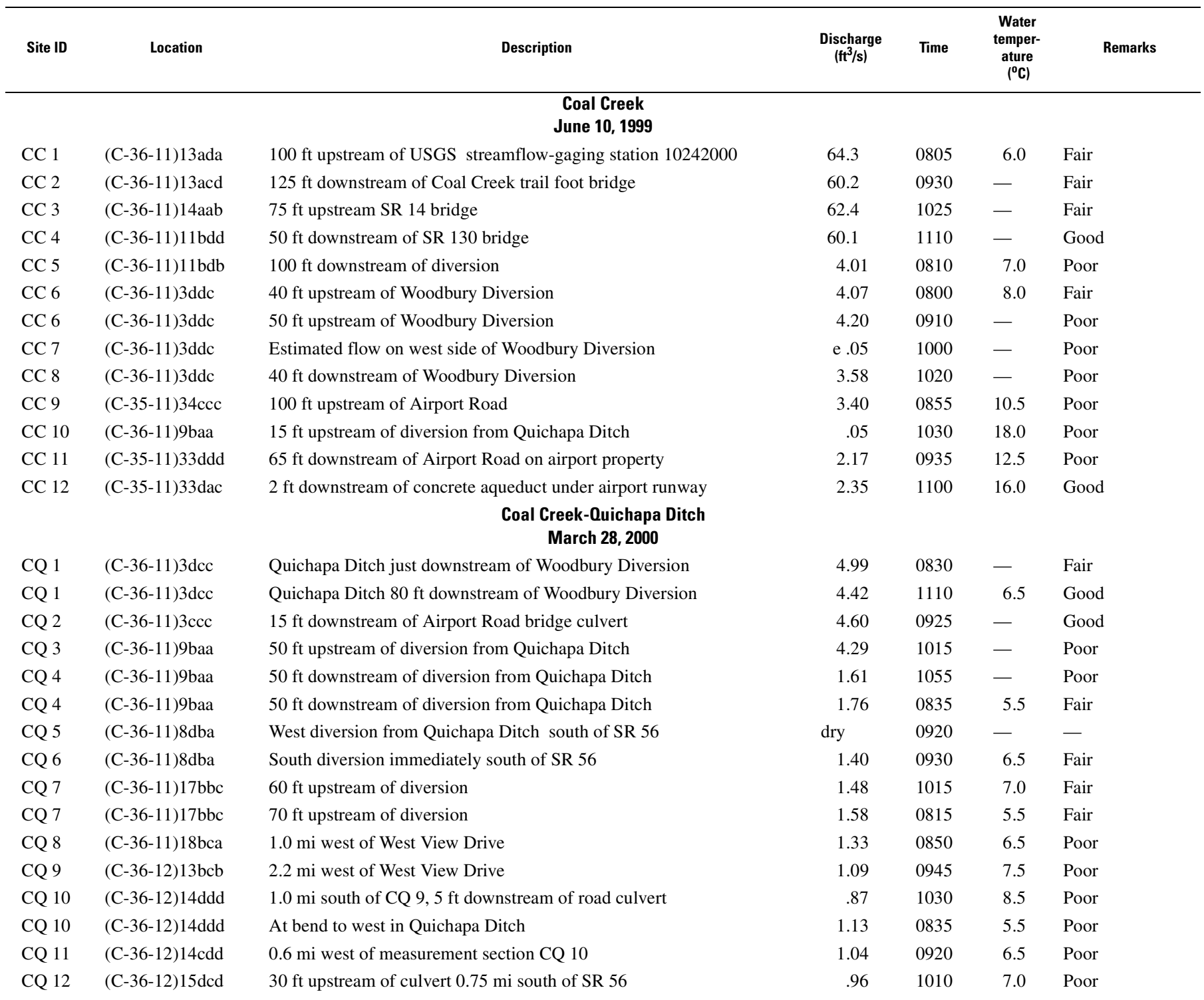


Table 5. Discharge, time of measurement, and temperature of water from Coal Creek, Quichapa Ditch, and Shirts Creek, Cedar Valley, Iron County, southwestern Utah-Continued

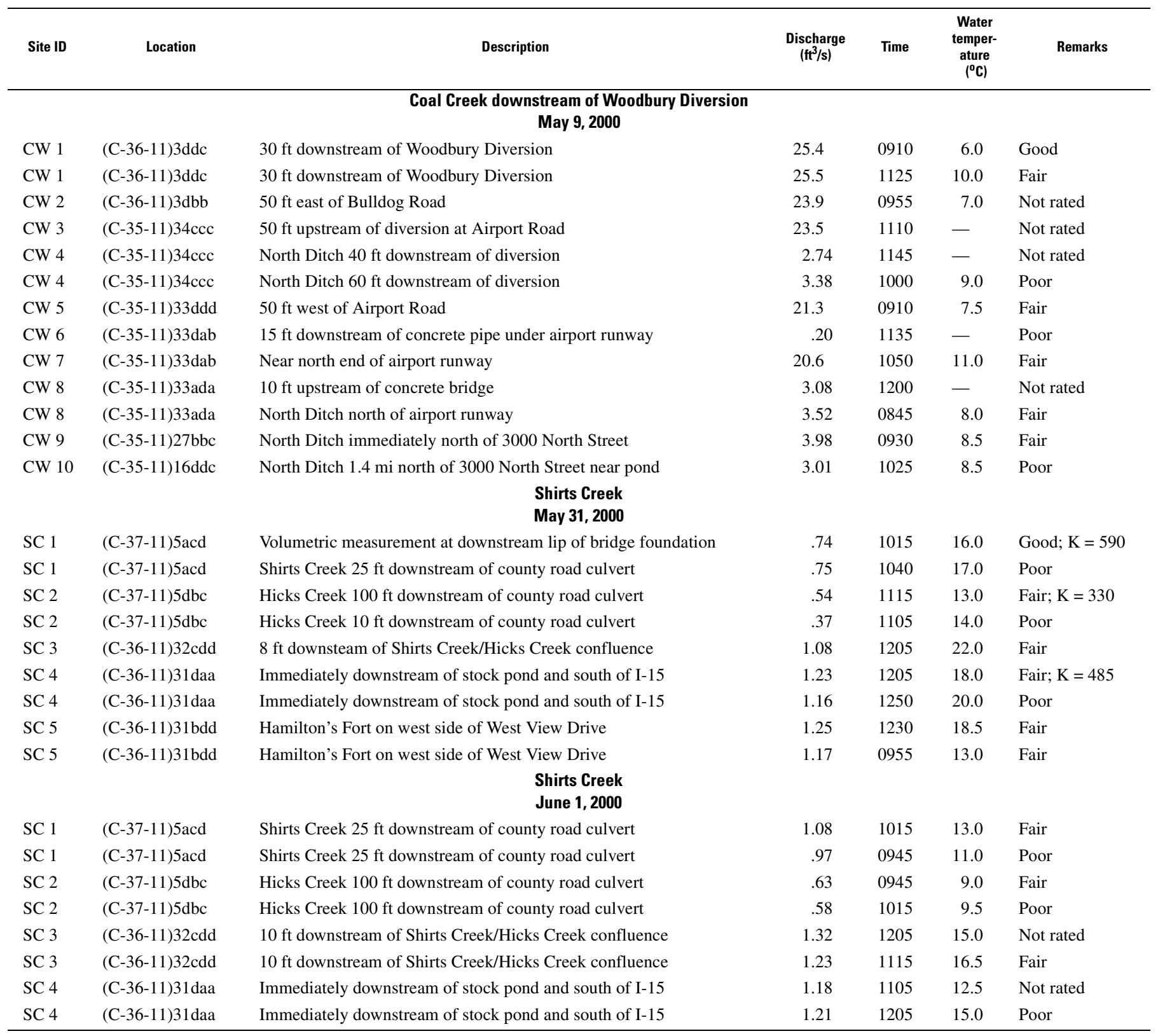

